\title{
STABILITY OF A COASTAL UPWELLING FRONT OVER TOPOGRAPHY
}

\author{
by \\ John Alexander Barth \\ B.A., University of Colorado \\ (1982) \\ SUBMITTED IN PARTIAL FULFILLMENT OF THE \\ REQUIREMENTS FOR THE DEGREE OF \\ DOCTOR OF PHILOSOPHY \\ at the \\ MASSACHUSETTS INSTITUTE OF TECHNOLOGY \\ and the \\ WOODS HOLE OCEANOGRAPHIC INSTITUTION
}

October 1987

(C)John A. Barth 1987

The author hereby grants to MIT and WHOI permission to reproduce and distribute copies of this thesis document in whole or in part.

Signature of Author

Joint Program in Oceanography, Massachusetts Institute of Technology/Woods Hole

Oceanographic Institution

Certified by

Kenneth H. Brink

Thesis Supervisor

Accepted by

Joseph Pedlosky

Chairman, Joint Committee for Physical Oceanography, Massachusetts Institute of Technology/Woods Hole

Oceanographic Institution 



\title{
STABILITY OF A COASTAL UPWELLING FRONT OVER TOPOGRAPHY \\ by \\ John Alexander Barth
}

\author{
Submitted to the Massachusetts Institute of \\ Technology/Woods Hole Oceanographic Institution \\ Joint Program in Oceanography \\ in October, 1987 in partial fulfillment of the \\ requirements for the Degree of Doctor of Philosophy
}

\begin{abstract}
A two-layer shallow water equation model is used to investigate the linear stability of a coastal upwelling front. The model features a surface front near a coastal boundary and bottom topography which is an arbitrary function of the cross-shelf coordinate. By combining the various conservation statements for the global properties of the system, a general stability theorem is established which allows the a priori determination of the stability of a coastal upwelling front.

Unstable waves are found for the modelled coastal upwelling front. The unstable wave motions are frontally-trapped and dominant in the upper layer. The wave propagates phase in the direction of the basic state flow and the primary energy conversion is via baroclinic instability. The effect of varying the model parameters is presented. Moving the front closer than $\sim 2$ Rossby radii to the coastal boundary results in a decrease in the growth rate of the fastest growing wave. Increasing the overall vertical shear of the basic state flow, by either decreasing the lower layer depth or increasing the steepness of the interface, results in an increase in the growth of the fastest growing wave.

A bottom sloping in the same sense as the interface results in a decrease of the growth rates and alongfront wavenumbers of the unstable waves in the system. Linearized bottom friction is included in the stability model and results in a decrease in the growth rates of the unstable waves by extracting energy from the system. Since the unstable mode is strongest in the upper layer, bottom friction will not stabilize the upwelling front.

A comparison between the predictions from the simple two-layer model and observed alongfront variability for three areas of active upwelling is presented. Reasonable agreement is found, suggesting that observed alongfront variability can be interpreted in terms of the instability of a coastal upwelling front.
\end{abstract}




\section{ACKNOWLEDGEMENTS}

I thank Ken Brink for his excellent advise, his ability to point out the important aspects of a particular problem and, above all, his efforts to develop my skills of scientific inquiry while respecting my ideas and inputs as he would those of a colleague. The members of my committee, Bob Beardsley, Dave Chapman, Joe Pedlosky, Jim Price, Paola Rizzoli and Bill Young, made many useful suggestions which improved this study. Conversations with Bill Young and Joe Pedlosky, in particular, helped develop my understanding of the physical processes discussed in this thesis.

Brian Petrie of the Bedford Institute of Oceanography generously provided the satellite infrared imagery from the Nova Scotia region. John Taunton-Clark of the Sea Fisheries Research Institute of South Africa kindly supplied the sea surface temperature maps from off southwestern Africa.

The friendship and support of my fellow Joint Program students and their spouses has been invaluable. They have made my time in the program most enjoyable. The long-standing support and interest of my parents have always made it easier to accomplish my goals. Most of all, I'd like to thank Carolyn for her companionship, her patience during the periods when my work became all-important and her constant belief in my ability to succeed.

This study was supported by the National Science Foundation Grant OCE 84-08563 and the Office of Naval Research Coastal Ocean Sciences Program 10/1984.37. 


\section{Contents}

$\begin{array}{lll}1 \text { Introduction } & 7\end{array}$

2 Background $\quad 13$

3 Model Description 2

4 Conservation Statements and a Stability Theorem 32

4.1 Introduction . . . . . . . . . . . . . . 32

4.2 Conservation Statements . . . . . . . . . . 33

4.3 Stability Theorem ................ 45

5 Results $\quad 51$

5.1 Introduction .................. 51

5.2 Solution Technique ................ 52

5.3 Inviscid, Flat-Bottom Model . . . . . . . . . . . 64

5.4 Inviscid, Sloping Bottom Model . . . . . . . . . . . 93

5.5 Linearized Bottom Friction . . . . . . . . . . . . 105

5.6 Summary . . . . . . . . . . . . . . . . 114

6 Comparison with Observations 116

6.1 Introduction . . . . . . . . . . . . . 116

6.2 Oregon ....................... 117

6.3 Nova Scotia . . . . . . . . . . . . 137

6.4 Southwestern Africa ................ 146 
6.5 Summary ...................... 155

7 Discussion $\quad \mathbf{1 5 7}$

7.1 Introduction . . . . . . . . . . . . 157

7.2 Simplifications and Omissions ............ 157

7.3 Implications .................... 163

8 Conclusions $\quad 165$

$\begin{array}{lr}\text { Appendix A } & 169\end{array}$

$\begin{array}{lr}\text { References } & 182\end{array}$ 


\section{Chapter 1}

\section{Introduction}

Fronts, regions of sharp, horizontal contrast in some fluid property (e.g. temperature, salinity or density), are a common feature in the ocean and atmosphere. A drastic change in air temperature from one day to the next is often the result of an atmospheric temperature front passing overhead. In the ocean, density fronts are associated with large scale jets such as the Gulf Stream and Kuroshio as well as with convergence zones which can span ocean basins. Near coastal barriers, several types of fronts can be identified. A layer of light water may flow alongshore next to a coast, forming a coastal current separated from the waters offshore by a density front. An example of such a current is the Norwegian coastal current. A surface to bottom density front may separate water masses on and off the continental shelf, an example of which is the shelfbreak front off New England in the Middle Atlantic Bight. Another type of front that can form in the coastal ocean is due to the process of upwelling. An alongshore wind directed so that the coastal barrier is to its left in the northern hemisphere drives an offshore Ekman flux in the upper part of the water column. This offshore flux requires some combination of horizontal and vertical flow to conserve the volume of seawater. The resulting sharp, near-surface horizontal density contrast between the less dense surface water offshore and the newly upwelled water inshore is called the coastal upwelling front. 
Upwelling occurs in many coastal regions of the world's oceans including the western coast of North America, southwest and northwest Africa, Peru and Nova Scotia. An example of a vertical section of density obtained off the coast of Oregon during the upwelling season (April-August, a time of persistent winds from the north-northwest) is shown in Fig. 1.1. The coastal upwelling front is readily identified as the region of compressed density contours intersecting the surface approximately $10 \mathrm{~km}$ offshore and continuing seaward at approximately $15-20 \mathrm{~m}$ depth. If alongshore winds were steady and the coastline and bottom topography uniform in the downwind direction, the coastal upwelling front would tend to form parallel to the coastline. Horizontal maps of surface properties (usually temperature because of its relative ease of measurement) often indicate a great deal of alongfront (the direction parallel to the front) variability in the offshore position of the front. An example of this alongfront variability is revealed in a map of sea surface temperature (SST) obtained from an airborne radiometer off the coast of Oregon (Fig. 1.2). The coastal upwelling front is the area of compressed isotherms approximately $20 \mathrm{~km}$ offshore with cold, upwelled water lying closest to shore. There exists a wave-like meander in the front with an alongfront wavelength of $30-50 \mathrm{~km}$. Alongfront variability on these scales is a common feature of coastal upwelling fronts. Other observations suggest that these disturbances can extend alongshore over many repeated wavelengths (Breaker and Mooers, 1986) and that the amplitude of the meanders can grow with time (Petrie et al., 1987). While several mechanisms can be suggested to explain alongfront variability in the coastal upwelling front, for example the influence of variations in the alongshore topography and/or bathymetry, their wavelike nature and observed growth suggest that they may be unstable waves which amplify on the front in the absence of external forcing.

Fronts are an important feature of the coastal ocean and an understanding of their variability is essential to a complete description of coastal 


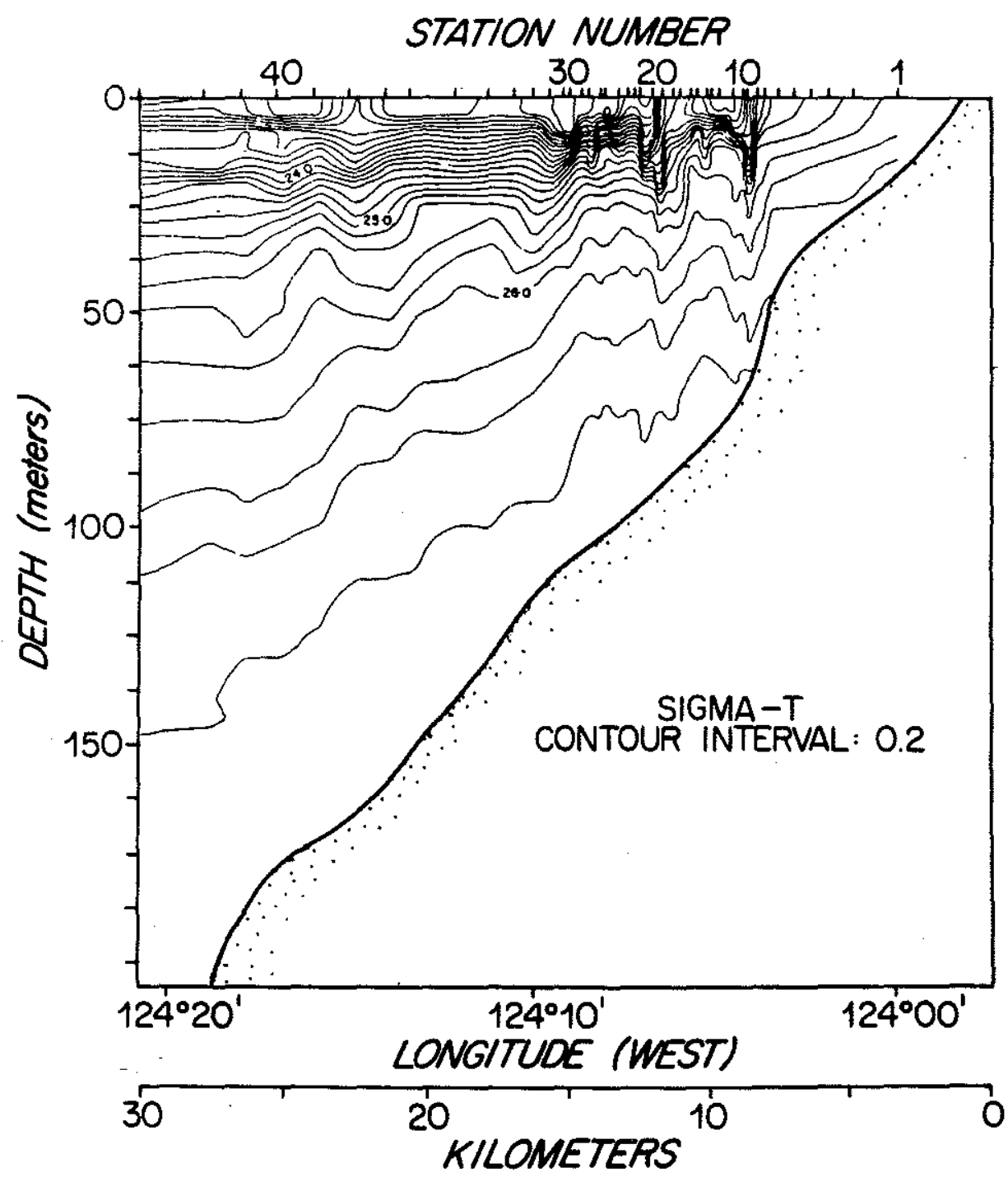

W-TRAVERSE TIME: 38 HOURS-

Figure 1.1: A density section near $45^{\circ} 15^{\prime} \mathrm{N}$ off the coast of Oregon during July, 1973. The region of compressed isopycnals intersecting the surface approximately $10 \mathrm{~km}$ offshore and continuing seaward at approximately $20 \mathrm{~m}$ depth is the coastal upwelling front. From Curtin (1979). 


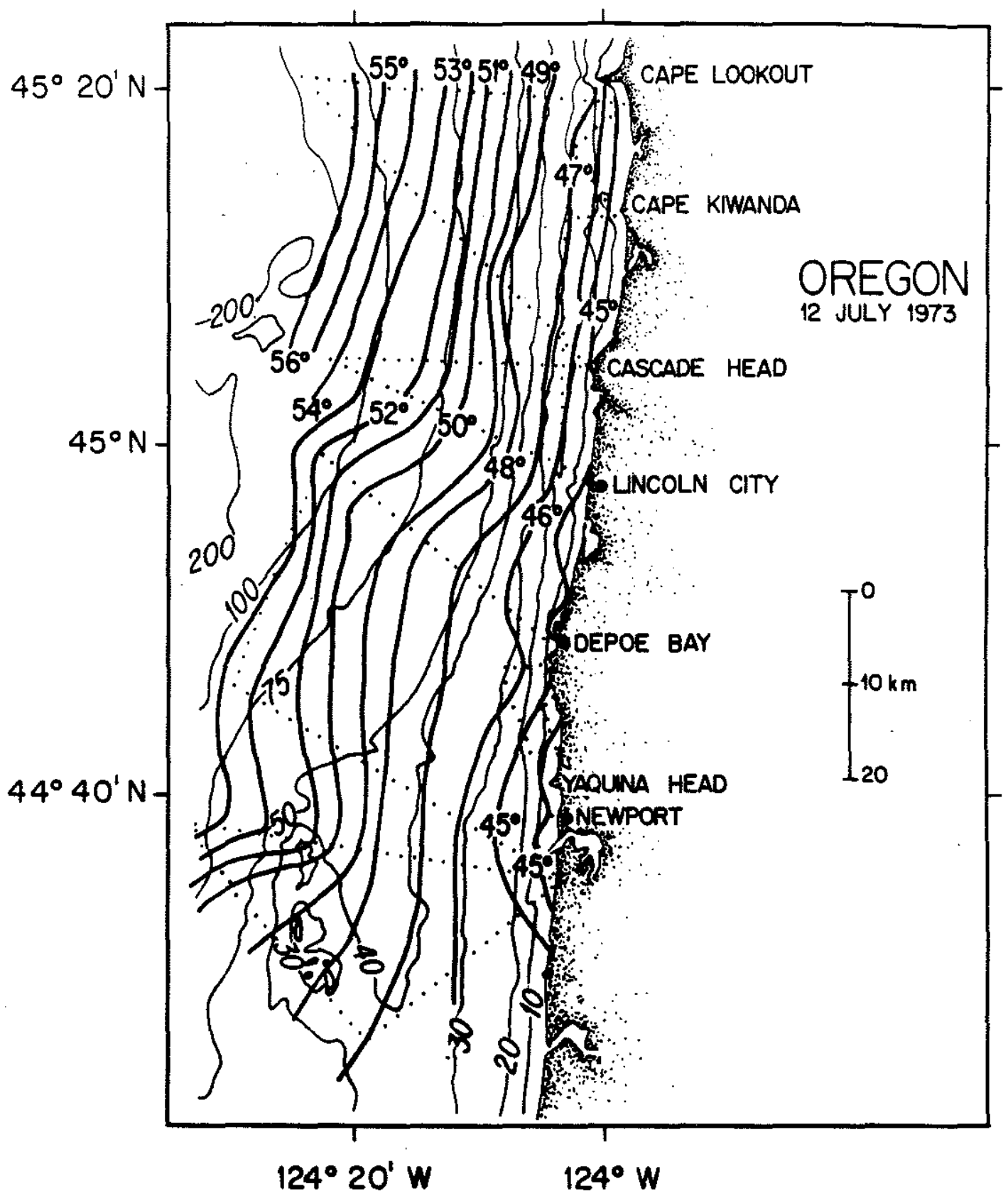

Figure 1.2: Sea surface temperature map off the coast of Oregon derived from aircraft radiometer measurements taken during July 14, 1973. Temperature contours are in degrees Fahrenheit and isobaths are in fathoms $(1 \mathrm{fm}=1.83 \mathrm{~m})$. The coastal upwelling front is the area of compressed isotherms approximately $20 \mathrm{~km}$ offshore. From O'Brien et al. (1974). 
circulation. Fronts in density and other water properties such as nutrient content play an important role in the biology of the coastal environment. Large amplitude meanders in the coastal upwelling front direct the normally alongshelf coastal flow cross-shelf and may, with time, grow to the point where they break the continuity of the alongshelf flow by forming detached eddies and contribute significantly to the cross-shelf transport of water properties. Clearly, it would be useful to understand the formation of these frontal meanders and to be able to predict their alongfront length scale, rate of growth and other relevant properties.

The goal of this study is to show that wave-like disturbances with the properties of observed frontal variability can be produced by hydrodynamic instability. The approach will be to examine the potential for unstable disturbances to form on a basic state flow which has presumably arisen previously from the influence of an alongshore wind via traditional upwelling processes. Small amplitude, hence linear, disturbances of normal mode form (periodic wave form in space with the possibility of exponential growth in time) are examined. In this study, the dynamics of shallow, rotating layers of homogeneous incompressible fluids are considered. The governing equations employed are the shallow water equations rather than the quasigeostrophic equations (Pedlosky, 1986) because the latter, while simplifying the instability calculation, are inapplicable to frontal regions. Large interface displacements, strong horizontal shears and large slopes in the bottom topography (which are allowed below) are not allowed in quasi-geostrophic theory. The inclusion of ageostrophic dynamics will substantially modify the well known results of quasi-geostrophic stability theory.

A review of relevant frontal instability models will be presented first, followed by a description of the particular model employed in this study. In the latter chapter relevant parameters and symbols will be defined, the governing equations will be stated and an approximation used to simplify the numerical solution technique will be described. Next, conservation state- 
ments are derived and used to obtain general stability criteria. Following this, results from a numerical solution for a variety of basic states and geometries will be presented. Next, a comparison of the model calculations to observations from several upwelling regions will be presented. Finally, a discussion chapter is presented and conclusions summarized. 


\section{Chapter 2}

\section{Background}

Fronts in the ocean are of interest for several reasons. They are often regions of large velocities and velocity gradients which are fundamental to the structure of the circulation. Fronts often separate different water masses and are certainly important in biological processes. This study does not concern the formation, internal dynamics or observational description and classification of oceanic fronts. For a general review of fronts in the coastal ocean see Bowman and Esaias (1978), Richards (1981) or Simpson and James (1986). The formation of fronts has been studied extensively especially in the atmosphere. A discussion of frontogenesis in the coastal ocean can be found in the above references and a thorough study of atmospheric frontogenesis can be found in the work of Hoskins and Bretherton (1972). Models of the formation of the coastal upwelling front are of particular interest because they provide the basic state density and velocity fields whose stability are investigated in this study. A brief review of relevant models is presented below. The dynamics and evolution of frontal regions whose initial structure is assumed known has been studied extensively (e.g. Garvive, 1978ab and 1980). Recently, Cushman-Roisin (1986) has developed a formalism to study frontal geostrophic dynamics. A large body of observational work on fronts exists. Studies relevant to the coastal upwelling front include Curtin (1979), Mooers et al. (1976) and Stevenson et al. (1974). 
The formation of the coastal upwelling front has been studied using a hierarchy of analytic models. The basic physical mechanism for frontal formation is described in chapter 1 and repeated here. An alongshore wind stress with the coast on its left in the northern hemisphere drives an offshore Ekman flux in the upper part of the water column. To conserve mass, water is upwelled from below creating a density front between the dense inshore water and the lighter offshore water. Csanady $(1971,1977)$ considered the formation of a coastal upwelling front in an inviscid two-layer model. After the action of an impulsive alongshore wind stress, a steady solution was found by conserving potential vorticity in each layer. The interface rises to an exponential profile over a scale equal to the internal Rossby radius of deformation and intersects the sea surface some specified distance offshore. Pedlosky $(1978 \mathrm{a}, \mathrm{b})$ developed a nonlinear inviscid model of upwelling in a continuously stratified fluid. He modelled the offshore Ekman flux of fluid in the upper part of the water column as a line sink of fluid at the coast. The equilibrium solutions obtained by Pedlosky (1978a) have density surfaces rising to contact the surface and a length scale again given by the internal Rossby radius of deformation. The density surfaces in a model with linear stratification all intersect the surface at the coast thus forming a sharp horizontal density contrast (a front). The time-dependent nonlinear solutions in Pedlosky (1978b) illustrate the sharpening of the horizontal density gradients over scales less than the Rossby deformation radius. A major deficiency of the latter two models is the replacement of the link between the inertial interior flow and the frictional surface Ekman layer by the line sink of fluid at the coast. This results in the surface front not being able to move offshore as is observed in nature (Mooers et al., 1976).

In an alternative approach, de Szoeke and Richman $(1981,1984)$ include a simple parameterization of vertical mixing processes in a two-layer model of wind-driven coastal upwelling. They include entrainment between the two layers due to wind mixing which keeps the upper mixed layer from dis- 
appearing and also allows denser deep water to surface. This entrainment process permits horizontal density contrasts to form away from the coast in the upper mixed layer. In response to an alongshore wind stress the pycnocline initially rises over a distance equal to the Rossby radius of deformation to contact the surface adjacent to the coast. This coastal upwelling front is then advected offshore leaving behind a thin $0(1 \mathrm{~km})$ coastal zone of active upwelling. The width of the front itself is found to be $0(100 \mathrm{~m})$ in agreement with the sharp fronts observed in nature (Mooers et al., 1976). Recently, Rudnick and Davis (1987) have formulated a Lagrangian theory of frontogenesis in mixed layers for a variety of entrainment parameterizations. The model describes the formation of fronts given a specified cross-front velocity field. For a velocity field associated with coastal upwelling the mixed layer shoals over a distance equal to the Rossby deformation radius and eventually forms a thin front which is advected offshore much as in the model of de Szoeke and Richman (1984).

While the details of the results from each of these models differ, they each describe the formation of a density front which intersects the surface of the ocean. The scale over which the interface or density surfaces warp upward is generally the internal Rossby radius of deformation. The mixed layer models are able to reproduce the offshore migration of the surface front, a feature which is observed in nature.

Another approach to describing the formation of coastal upwelling fronts is through the use of numerical models. These include processes omitted in the simpler analytic models described above. They serve to provide details about the structure of the frontal region both in density and velocity but the essential results described above remain valid. See Brink (1983) for a review and reference list of some relevant numerical models.

Another direction frontal studies have taken is to assume that the basic frontal shape is known and investigate the possible existence of disturbances on this mean state. The form of the disturbance is usually postulated to be 
wave-like and can either be stable (constant amplitude in time) or unstable (amplitude grows with time). The stable modes of oceanic fronts have been studied using various model basic states. For the most part, these studies considered the dynamics of one or two homogeneous layers. Garvine (1983) and Paldor (1983a) used similar linear, reduced gravity models with uniform potential vorticity in the upper layer to obtain stable, frontallytrapped waves. Garvine's (1983) model included a thin dissipative zone adjacent to the surface front where turbulent entrainment may occur. He did not describe the details of the motion in this dissipative zone and only considered it as a source or sink of fluid for the inviscid region away from the surface front. Garvine (1983) obtained only waves with zero phase speed because of the requirement that there be no horizontal shear of the alongfront velocity at the boundary between the inviscid interior flow and the viscous region near the surface front. He also stipulated that there be no cross-stream flow between the inviscid interior and the dissipative zone. Paldor (1983a) considered inviscid dynamics throughout the frontal region and obtained wave solutions which propagated both in the direction of and against the mean flow. Garvine (1984), in an extension of the reduced gravity model described above, allowed the exchange of fluid between the inviscid interior flow and the viscous frontal zone. A finite cross-stream flow allows nonzero horizontal Reynolds stresses which contribute to the change in time of the wave kinetic energy. He obtained waves which grew spatially in the downstream direction when the dissipative zone entrained water from below and fed cross-stream flow into the inviscid interior and decaying waves for the reverse.

Several authors have included the effect of an active lower layer in studies of stable frontal waves. Bane and Hsueh (1980) considered a special geometry with both the interface and the bottom profile linearly sloping away from the coast. For the "upwelling" case where the interface is allowed to warp upward and intersect the surface offshore they obtain a stable wave 
which they call a "complementary-mode edge wave". This mode propagates in the same sense as topographic waves (with the coast to the right in the northern hemisphere) and consists of a barotropic oscillation inshore of the front coupled to an oscillating interface offshore. Their basic state flow is potentially unstable (see chapter 4), but the authors concentrated solely on the stable modes of the system. Bane (1980) considered a two-layer model with arbitrary interface and bottom geometries and in addition to the complementary-mode edge wave found a stable frontally-trapped wave which propagated in the same sense as topographic waves. Finally, Luther and Bane (1980) used a continuously stratified model with arbitrary current/density structure and bottom topography to examine these same types of stable waves.

The above studies provide a description of the types of waves which may be important in considering the stability of a coastal upwelling front. The one-layer results of Paldor (1983) suggest that frontally-trapped waves which propagate in either alongfront direction may be important in the instability process. For a two-layer fluid with a flat bottom one might anticipate the existence of a wave solution which is frontally-trapped, but has comparable magnitudes in the two layers (a more "barotropic" mode). With bottom topography, familiar vorticity wave modes including shelf waves and the complimentary-mode edge waves of Bane and Hsueh (1980) and Bane (1980) are introduced.

The study of unstable waves on oceanic density fronts relies quite heavily on earlier quasi-geostrophic instability models. These earlier studies provide a basic understanding of instability processes and a theoretical framework upon which the frontal studies have built. The classic quasigeostrophic baroclinic instability studies of Charney (1949), Eady (1949), Fjørtoft (1951) and Phillips (1954) detail the mechanism by which energy is transferred from the potential energy of the mean flow to the growing disturbance. Studies such as Pedlosky (1964) and Killworth (1980) provide 
useful criteria for determining whether a particular mean flow will be unstable or not. Since the velocity fields associated with fronts consist of both horizontal and vertical shear, barotropic instability, the process of energy conversion from the mean kinetic energy to the growing waves, can also be important. Studies of this type of instability have a long history going back to Rayleigh (1880) with more recent contributions by Kuo (1949 and 1973) and Howard and Drazin (1964). An excellent summary of quasi-geostrophic instability appears in Pedlosky (1986).

However, for the reasons given in the first chapter, quasi-geostrophic theory is clearly inapplicable to the study of frontal instability. One of the first studies of frontal instability using the shallow water equations was that of Orlanski (1968). He studied a two-layer Margules front intersecting flat top and bottom boundaries and explored a wide range of Rossby number-Richardson number space finding unstable waves at all wavelengths. Orlanski (1969) extended the model to include arbitrary interface and bottom profiles with the goal of modelling unstable waves in the Gulf Stream. He found that bottom topography plays an important role in determining the properties of the unstable waves. The presence of sloping bottom topography was found to stabilize (decrease the growth rates of the unstable waves, but not eliminate them) the system in agreement with quasi-geostrophic results (e.g. Mechoso and Sinton, 1981). Orlanski concentrated on the Gulf Stream problem (small Rossby number flows) and did not model a surface front over a continental shelf near a coast such as the coastal upwelling front.

Many recent studies of frontal instability have employed a one-layer reduced gravity model to simplify the mathematics. Killworth and Stern (1982) studied a model of a coastal current with a pool of light water lying next to a coastal barrier (Fig. 2.1a). They showed that for this onelayer wall-bounded front, unstable waves exist even if the potential vorticity of the basic state is monotonic in the cross-front direction. A necessary 

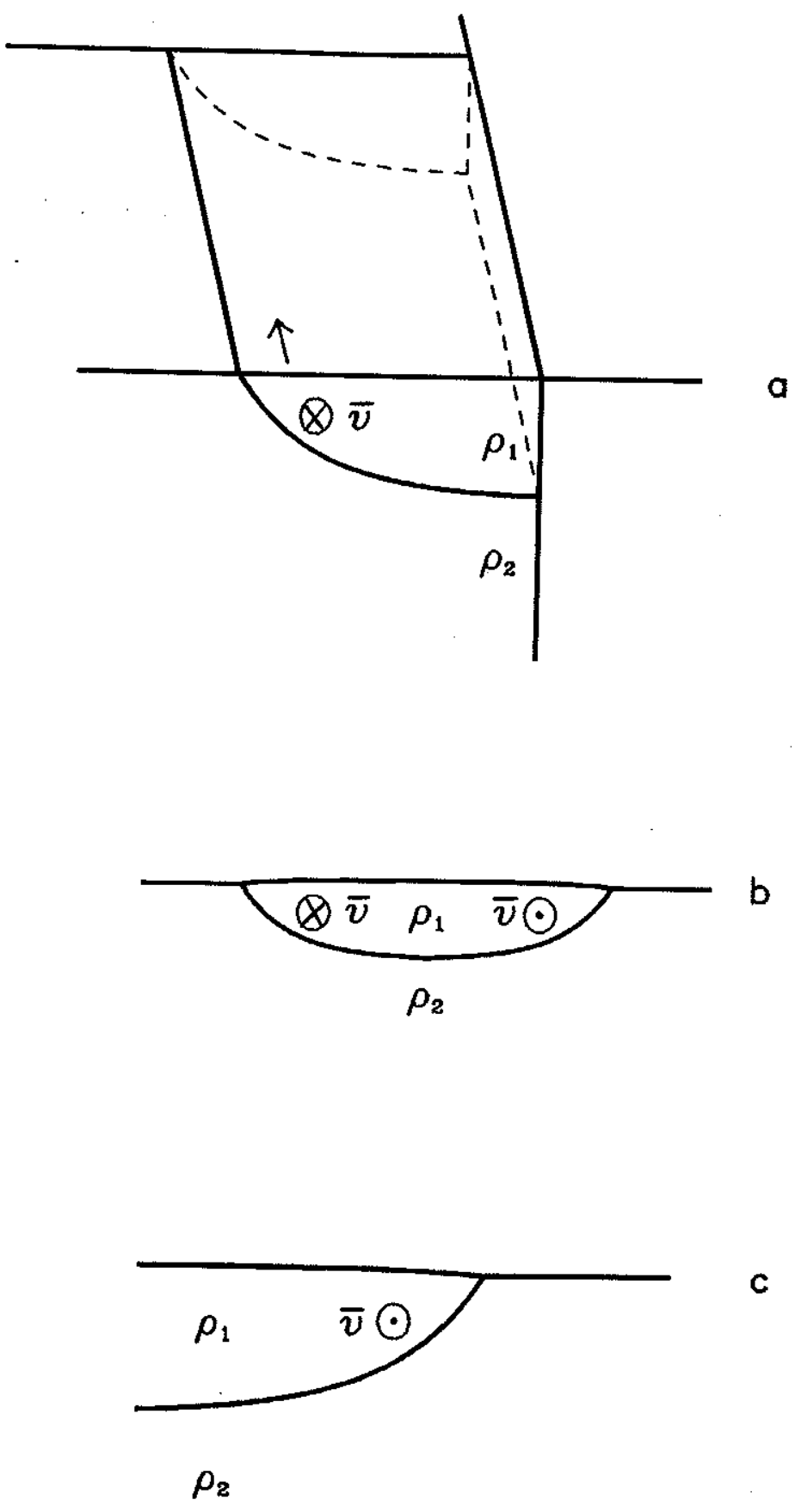

Figure 2.1: Examples of reduced gravity, frontal instability model geometries. (a) Coastal current. (b) Two-front current. (c) Isolated front. 
condition for instability from quasi-geostrophic theory is that the basic state potential vorticity gradient must change sign somewhere in the fluid. The monotonic potential vorticity model of Killworth and Stern (1982) clearly does not satisfy this criteria yet still yields unstable waves. This difference from quasi-geostrophic theory will be expounded upon further in chapter 4. The stability of a coastal current has also been investigated by Paldor (1983b) and Kubokawa (1986). The nonlinear long wave flow of a coastal current was studied by Stern (1980) who found solutions representing bores, blocking and breaking waves.

A reduced gravity model with two fronts (Fig. 2.1b) has also received attention in the study of frontal instability. Using a $f$-plane version of this model, Griffiths et al. (1982) found an unstable mode on a basic state with zero potential vorticity due to the resonance of two waves each trapped to a separate front. In addition, a high wavenumber weak instability was found which involved one front only. Recently Hayashi and Young (1987) have investigated the stability of a one-layer, two-front model on an equatorial $\beta$-plane and found unstable modes. Finally, Paldor (1986) found special nonlinear solutions on a two-front model which represent solitons. These waves merely propagate with time and do not grow temporally. He considers long waves only and the role of nonlinearity on the stability of the system with dispersion present is still an open question.

Noting the unstable mode growing on a single front with no apparent interaction with the other front in the two-front model of Griffiths et al. (1982), Killworth (1983) relaxed the uniform potential vorticity requirement used by Paldor (1983a) in a study of a one-layer reduced gravity model of an isolated front (Fig. 2.1c). He showed analytically that the front may be unstable if the upper layer's depth tends to its value at infinity more rapidly than the uniform potential vorticity front of the same depth. Kubokawa (1985), using the same isolated front model, extended the longwave results of Killworth (1983) to finite wavenumbers and did a 
detailed analysis of the energy transfers from the basic state to the unstable waves. In both studies the unstable mode was weak (i.e. growth rate in time very small) and the basic state potential vorticity gradient did not change sign.

These reduced gravity models leave out the effect of an active lower layer on the stability of a front. Two active layers allow for the possibility of true baroclinic instability. Many laboratory experiments have explored the instabilities associated with fronts (e.g. Stern et al., 1982; Griffiths et al., 1982; Griffiths and Linden, 1982; Chia et al., 1982; and Narimousa and Maxworthy, 1985). These laboratory models, which all have active lower layers, exhibit growth rates much larger than those found by Killworth (1983). A further reason to include an active lower layer is to study the effect of bottom topography. An active lower layer can also provide damping to the system via bottom friction. Further, for a model of the coastal upwelling front the lower layer velocity must satisfy the boundary condition of no flow through the coastal barrier. Killworth et al. (1984) studied a two-layer isolated front with a flat bottom. They were able to show analytically that for long waves and deep lower layers an unstable mode exists no matter what the distribution of basic state potential vorticity. This mode has growth rates of the same magnitude as observed in the laboratory models. They extended their analytic result to finite wavenumbers numerically and briefly commented on the energy transfer between the mean flow and the growing waves.

To model a coastal upwelling front correctly, a coastal barrier and bottom topography must be included. These topographic features will be included in the current study, the details of which are given in the following chapter. The previous models show that shallow lower layers destabilize while sloping bottom topography may stabilize the system. Since the coastal upwelling front environment contains both these features, it will be interesting to investigate the net effect on the stability of the basic state 
flow. This study will also include an examination of the energy transfers in the system which have not received adequate attention in the previous studies of frontal stability. In addition, general criteria for a priori determination of the stability of a given flow will be developed. This set of criteria will be used to recover the results of several of the previous models. Finally, since bottom friction has been shown to be an important process in the coastal ocean (e.g. Brink and Allen, 1978; Brink, 1982; Allen, 1984), the effect of a linearized form of friction on the stability of the system will be investigated. 


\section{Chapter 3}

\section{Model Description}

The model employed here is a simple, two-layer shallow water equation model with a rigid lid on an $f$-plane. The stability analysis will be carried out both in the inviscid case and with linearized bottom friction. The model explicitly leaves out the effects of wind stress and mixing. Even though these processes are known to be important in the formation of the coastal upwelling front (see chapter 2), their inclusion complicates the governing equations sufficiently that a full numerical model is required. Thus, this model investigates the stability of a coastal upwelling front which has previously arisen due to an alongshore wind stress as described in chapter 2. The applicability of a stability model without wind stress may be rationalized in the following two ways. First, coastal winds often become "upwelling-favorable" (blowing alongshore with the coast to the left in the northern hemisphere) for a period of a few days then relax or change direction (Huyer, 1983). Therefore, this instability model may be thought of as formally applying after one of these upwelling events. Second, the model may be appropriate even in the presence of a wind stress. In the real ocean, dissipation (e.g. via interfacial friction) will provide a sink of energy so that the wind-forced system may reach a steady state. If the dissipation is strong enough to effect this balance but weak enough to leave the structure of the unstable waves essentially unchanged, then the wind 
stress will only affect the stability analysis indirectly through its effects on the mean flow field. Since the wind forcing does not directly enter the stability calculation, an unforced model may be appropriate. However, as commented on further in chapter 7 , time-dependence in the basic state flow field as forced by a time-dependent wind stress (or for a steady wind stress before a steady-state is established) may affect the stability properties of the system.

The model geometry is shown in Fig. 3.1. Two homogeneous layers of density $\rho_{1}$ and $\rho_{2}\left(\rho_{2}>\rho_{1}\right)$ lie adjacent to a coastal barrier. The origin of the coordinate system is chosen to be at the coast with $z$ in the vertical direction, $x$ in the cross-front direction (positive onshore) and $y$ in the alongfront direction. The entire system is rotating about the $z$-axis with an angular frequency $f / 2$ where $f$ is the Coriolis parameter. The layer thicknesses are denoted by $h_{1}$ and $h_{2}$, while the bottom topography, which is an arbitrary function of $x$ but assumed uniform in $y$, is given by $H=h_{1}+h_{2}$. The sea surface elevation is denoted by $\zeta_{1}$. The surface front, modelled as the interface between the layers of different densities, lies parallel to the coast at the point $x_{f}\left(x_{f}<0\right)$, offshore of the coastal barrier. The sloping interface and bottom adjoin a flat-bottom region (representing the deep ocean offshore of the continental margin) with constant layer depths $\left(H_{1}, H_{2}\right)$ far removed from the surface front. A basic state alongfront flow $(\bar{v})$ which is uniform in $y$, independent of time $(t)$ and in geostrophic balance exists in the upper layer (Fig. 3.1b). For simplicity there is no basic state flow in the lower layer.

Before stating the governing equations, the field variables can be nondimensionalized as follows (e.g. Killworth et al., 1984):

$$
\begin{aligned}
\left(x_{*}, y_{*}\right) & =R(x, y) \\
\left(u_{1 *}, v_{1 *}, u_{2_{*}}, v_{2_{*}}\right) & =\left(g^{\prime} H_{1}\right)^{1 / 2}\left(u_{1}, v_{1} u_{2}, v_{2}\right) \\
t_{*} & =f^{-1} t
\end{aligned}
$$




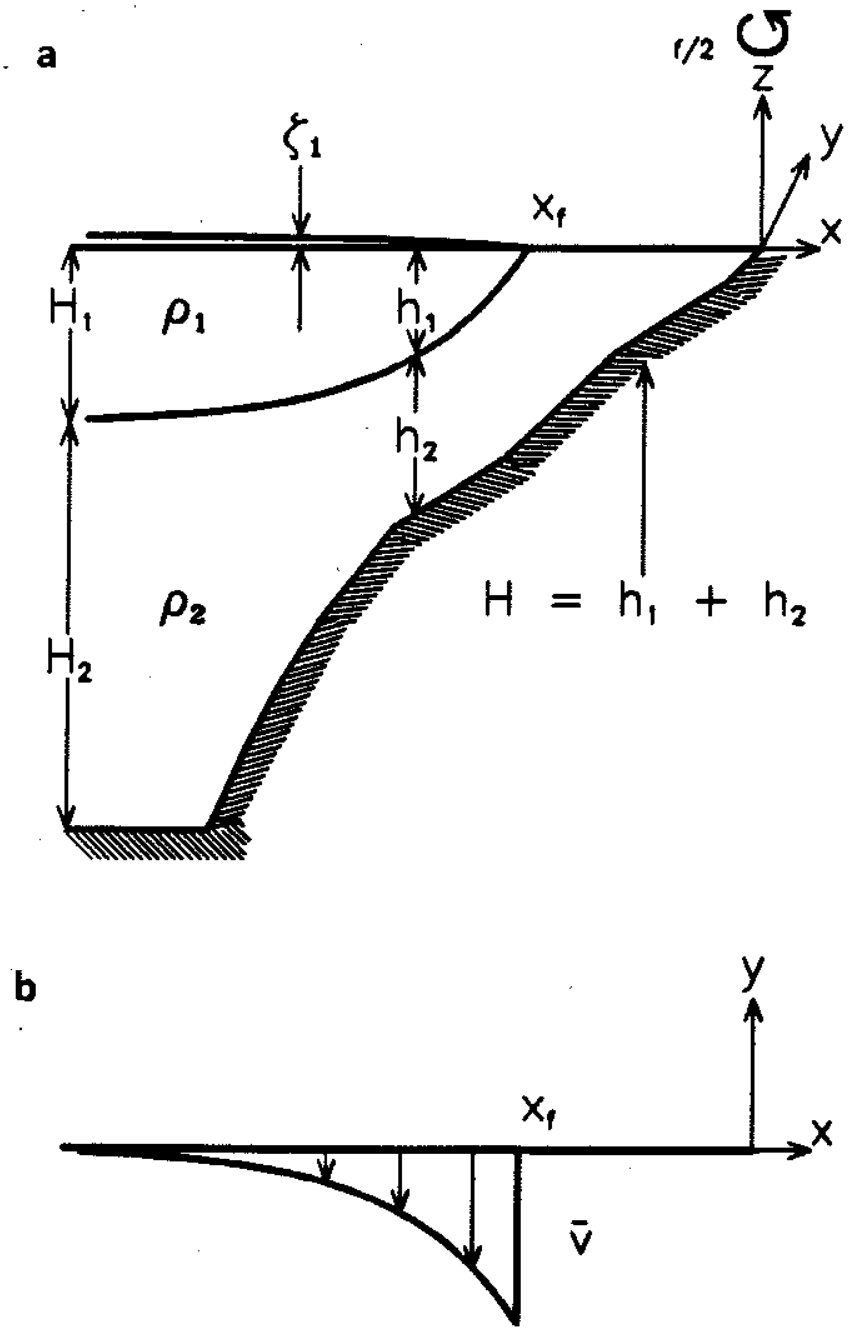

Figure 3.1: (a) Model geometry (symbols defined in text) and (b) upper layer basic state flow. 


$$
\begin{aligned}
\left(h_{1 *}, h_{2 *}\right) & =H_{1}\left(h_{1}, h_{2}\right) \\
\zeta_{1 .} & =\delta H_{1} \zeta_{1} .
\end{aligned}
$$

The variables subscripted with an asterisk are dimensional and the velocity ( $u$ cross-front, $v$ alongfront) subscripts indicate either the upper or lower layer. Horizontal length is scaled by the internal Rossby radius of deformation, $R=\left(g^{\prime} H_{1}\right)^{1 / 2} / f$, where the reduced acceleration due to gravity is given by $g^{\prime}=g\left(\rho_{2}-\rho_{1}\right) / \rho_{2}=g \delta$. Note that the density defect, $\delta$, is much less than one. For typical coastal upwelling fronts $R \sim 5-10 \mathrm{~km}$. Velocities are nondimensionalized by the internal gravity wave phase speed, $\left(g^{\prime} H_{1}\right)^{1 / 2}$, which is typically $50-100 \mathrm{~cm} \mathrm{~s}^{-1}$. Time is scaled by $f^{-1}$. Note that the sea surface elevation and layer depths are scaled such that their nondimensional versions are of the same order.

The nondimensional, two-layer, inviscid shallow water equations are, using subscripts to denote partial differentiation,

$$
\begin{gathered}
\frac{D u_{1}}{D t}-v_{1}=-\zeta_{1_{x}}, \\
\frac{D v_{1}}{D t}+u_{1}=-\zeta_{1_{y}}, \\
h_{1_{t}}+\left[u_{1}\left(h_{1}+\delta \zeta_{1}\right)\right]_{x}+\left[v_{1}\left(h_{1}+\delta \zeta_{1}\right)\right]_{y}=0
\end{gathered}
$$

for the upper layer and

$$
\begin{gathered}
\frac{D u_{2}}{D t}-v_{2}=-\zeta_{T_{x}}, \\
\frac{D v_{2}}{D t}+u_{2}=-\zeta_{T_{y}}, \\
-h_{1 \mathrm{t}}+\left(u_{2} h_{2}\right)_{x}+\left(v_{2} h_{2}\right)_{y}=0
\end{gathered}
$$

for the lower layer where

$$
\zeta_{T}=(1-\delta) \zeta_{1}-h_{1}
$$


is essentially the lower layer "pressure". Here

$$
\frac{D}{D t}=\frac{\partial}{\partial t}+u_{i} \frac{\partial}{\partial x}+v_{i} \frac{\partial}{\partial y}
$$

where $i=1,2$. Linearized bottom friction terms will be included in the latter part of chapter 5 .

To simplify the numerical solution technique the geostrophic momentum approximation (Hoskins, 1975 and Hoskins and Bretherton, 1972) is made. Specifically, the approximation will make the governing equations linear in the eigenvalue making the system easier to solve numerically. The approximation consists of replacing the fluid velocities (or momentum, hence the name) by their geostrophic values when acted upon by the substantial derivative given by (3.3). Specifically, the following substitutions are made:

$$
\begin{aligned}
& \frac{D u_{i}}{D t}=\frac{\partial u_{i_{g}}}{\partial t}+u_{i} \frac{\partial u_{i_{g}}}{\partial t}+v_{i} \frac{\partial u_{i_{g}}}{\partial t} \\
& \frac{D v_{i}}{D t}=\frac{\partial v_{i_{g}}}{\partial t}+u_{i} \frac{\partial v_{i_{g}}}{\partial t}+v_{i} \frac{\partial v_{i_{g}}}{\partial t}
\end{aligned}
$$

where $i$ again denotes either layer one or two and

$$
\begin{array}{rrr}
u_{1_{g}} & =-\zeta_{1_{y}}, \\
v_{1_{g}} & =\zeta_{1_{x}}, \\
u_{2 g} & = & -\zeta_{T_{y}}, \\
v_{2_{g}} & =\zeta_{T_{x}}
\end{array}
$$

are the geostrophic layer velocities. It is important to retain the full advecting velocities (geostrophic and ageostrophic) in (3.3). According to Hoskins (1975), the approximation is valid when the magnitude of the time rate of change of the velocity vector is small compared with the magnitude of the Coriolis force, or, dimensionally,

$$
\left|\frac{D \overrightarrow{v_{*}}}{D t_{*}}\right| \ll\left|f \overrightarrow{v_{*}}\right|
$$

Hoskins (1975) and Hoskins and Bretherton (1972) have shown that the approximation works well in frontal regions with large horizontal shear 
provided the curvature vorticity (the turning of the flow along a streamline) is not large.

Another way to develop the geostrophic momentum approximation is to substitute for the velocities acted upon by the substantial derivative in (3.1a) and (3.1b) from the velocities in the Coriolis terms in (3.1b) and (3.1a) to obtain

$$
\begin{gathered}
v_{1}=\zeta_{1_{z}}-\frac{D \zeta_{1_{y}}}{D t}-\frac{D^{2} v_{1}}{D t^{2}}, \\
u_{1}=-\zeta_{1_{y}}-\frac{D \zeta_{1_{x}}}{D t}-\frac{D^{2} u_{1}}{D t^{2}} .
\end{gathered}
$$

The lower layer equations (3.2) can be treated in a similar manner. The geostrophic momentum approximation is then obtained by neglecting the last term in each of the two equations. That is

$$
\frac{D^{2} v_{1}}{D t^{2}} \ll v_{1}
$$

and

$$
\frac{D^{2} u_{1}}{D t^{2}} \ll u_{1}
$$

While these inequalities may fail locally somewhere in the fluid, this study is concerned with analyzing the global behavior of the system. The stable and unstable (if present) waves represent global solutions and their properties can be obtained with this approximate set of equations. Confidence in the geostrophic momentum approximation comes from direct comparison to the shallow water equation model of Killworth et al. (1984) and the successful frontal studies of Hoskins and Bretherton (1972 and a series of later papers).

Rather than relying on some Lagrangian time scale for the rate of change of the velocity vector being longer than $f^{-1}$ as Hoskins (1975) suggests, it is useful to reexamine the ratios of terms in (3.4). Since most fronts are long in one direction and short in the other, the coordinate parallel to the surface front will be rescaled as $y_{*}=(R / \epsilon) y$ where $\epsilon \ll 1$. This small 
parameter will turn out to be $l$, where $l$ is the alongfront wavenumber. With this scaling (3.4) becomes

$$
\begin{aligned}
& v_{1}=\zeta_{1_{x}}-\epsilon^{2} \frac{D \zeta_{1_{y}}}{D t}-\epsilon^{2} \frac{D^{2} v_{1}}{D t^{2}} \\
& u_{1}=-\zeta_{1_{y}}-\frac{D \zeta_{1_{x}}}{D t}-\epsilon^{2} \frac{D^{2} u_{1}}{D t^{2}} .
\end{aligned}
$$

At lowest order, the flow is geostrophic in the alongfront direction. The 0 (1) equations have been called "semi-geostrophic" (Pedlosky, 1986). In order to obtain results at higher wavenumbers (shorter alongfront scales) it is necessary to include terms of $0\left(\epsilon^{2}\right)$. The geostrophic momentum approximation includes the term $D \zeta_{1 y} / D t$ in the alongfront momentum balance, but arbitrarily neglects the final terms in each of the two equations. The geostrophic momentum approximation will allow results to be found at high wavenumbers, but may be suspect because of this arbitrary truncation of the $0\left(\epsilon^{2}\right)$ equations. In this study, reliable results are found at all wavenumbers for a flow with uniform basic state potential vorticity in the upper layer. As discussed further in section 5.3, the geostrophic momentum approximation fails to accurately predict the growth rate of high wavenumber unstable modes when the flow field has an interior point where the absolute vorticity $\left(f+\bar{v}_{x}\right)$ vanishes. However, it will turn out that the properties of the fastest growing modes on all the model fronts studied here are accurately predicted using the geostrophic momentum approximation. In any case, this approximation should be used with caution due to the arbitrary way in which it is "derived" from the shallow water equations.

The geostrophic momentum equations belong to the set of so-called "intermediate" approximations because they include physics simpler than included in the shallow water equations but more complete than in the quasi-geostrophic equations. In particular, they retain the ageostrophic advection of the geostrophic velocity field and can thus be used in regions of large horizontal shear. The geostrophic momentum approximation relies 
on low frequencies so, as a result, gravity waves are filtered out of the system. To reiterate, the desire to make this approximation stems solely from the simplification it provides to the numerical solution technique.

From (3.4) under the geostrophic momentum approximation, the twolayer momentum equations are

$$
\begin{gathered}
\frac{D \zeta_{1_{y}}}{D t}+v_{1}=\zeta_{1_{x}}, \\
\frac{D \zeta_{1_{x}}}{D t}+u_{1}=-\zeta_{1_{y}}, \\
h_{1_{t}}+\left[u_{1}\left(h_{1}+\delta \zeta_{1}\right)\right]_{x}+\left[v_{1}\left(h_{1}+\delta \zeta_{1}\right)\right]_{y}=0
\end{gathered}
$$

for the upper layer and

$$
\begin{gathered}
\frac{D \zeta_{T_{y}}}{D t}+v_{2}=-\zeta_{T_{x}}, \\
\frac{D \zeta_{T_{x}}}{D t}+u_{2}=-\zeta_{T_{y}} \\
-h_{1_{t}}+\left(u_{2} h_{2}\right)_{x}+\left(v_{2} h_{2}\right)_{y}=0
\end{gathered}
$$

for the lower layer where $D / D t$ is again given by (3.3).

As is traditional in linear instability theory the nondimensional field variables are expanded into a basic state (denoted by an overbar) and a perturbation (primed quantities). For the upper layer

$$
\begin{aligned}
& h_{1}(x, y, t)=(1-\delta) \overline{h_{1}}(x)-\zeta_{2}^{\prime}(x, y, t), \\
& \zeta_{1}(x, y, t)=\overline{\zeta_{1}}(x)+\zeta_{1}^{\prime}(x, y, t) \text {, } \\
& u_{1}(x, y, t)=u_{1}^{\prime}(x, y, t) \text {, } \\
& v_{1}(x, y, t)=\bar{v}(x)+v_{1}^{\prime}(x, y, t)
\end{aligned}
$$

and for the lower layer

$$
\begin{aligned}
& h_{2}(x, y, t)=\overline{h_{2}}(x)+\zeta_{2}^{\prime}(x, y, t) \\
& \zeta_{T}(x, y, t)=\overline{\zeta_{T}}(x)+\zeta_{T}^{\prime}(x, y, t), \\
& u_{2}(x, y, t)= \\
& v_{2}(x, y, t)= \\
& u_{2}^{\prime}(x, y, t),
\end{aligned}
$$


Note that mean flow only exists in the upper layer. The basic state is uniform alongshore, independent of time and in geostrophic balance

$$
\bar{v}=\overline{\zeta_{1_{x}}}=\overline{h_{1_{x}}}
$$

Substituting these expressions into (3.5) and (3.6), linearizing about the basic state and dropping primes, the geostrophic momentum equations for the perturbations become

$$
\begin{gathered}
\zeta_{1_{y t}}+\bar{v} \zeta_{1_{y y}}+v_{1}=\zeta_{1_{x}}, \\
\zeta_{1_{x t}}+\bar{v} \zeta_{1_{x y}}+\left(1+\bar{v}_{x}\right) u_{1}=-\zeta_{1_{y}}, \\
-\zeta_{T_{t}}+\left(u_{1} \overline{h_{1}}\right)_{x}+\left[v_{1} \overline{h_{1}}+\bar{v}\left(\zeta_{1}-\zeta_{T}\right)\right]_{y}=0
\end{gathered}
$$

for the upper layer and

$$
\begin{gathered}
\zeta_{T_{y t}}+v_{2}=\zeta_{T_{x}}, \\
\zeta_{T_{z t}}+u_{2}=-\zeta_{T_{y}}, \\
\zeta_{T_{t}}+\left(u_{2} \overline{h_{2}}\right)_{x}+\left(v_{2} \overline{h_{2}}\right)_{y}=0
\end{gathered}
$$

for the lower layer where now

$$
\zeta_{T}=(1-\delta) \zeta_{1}+\zeta_{2}
$$

Note that in (3.8c) terms of $0(\delta)$ have also been ignored. While these equations will eventually be solved numerically for a variety of specific basic state flows, it is useful to examine them first to see if any general statements can be established. In the next chapter, conservation statements for several properties of the system are derived. These lead to general stability criteria which can be applied to systems governed by (3.8)-(3.10). 


\section{Chapter 4}

\section{Conservation Statements and a Stability Theorem}

\subsection{Introduction}

While the existence of unstable waves on a particular basic state flow can be determined numerically, it would be advantageous to have a general set of criteria for determining a priori whether that configuration is favorable for the growth of unstable disturbances. In this chapter, such criteria are developed through the use of global conservation statements for energy, momentum and potential vorticity. Most of the detailed derivations are contained in Appendix A. These conservation statements are also useful in the dynamical interpretation of the instability mechanism, since they help to elucidate the details of energy transfer in the system. It will also be interesting to point out how the form of the conservation statements derived using the geostrophic momentum equations differs from its quasigeostrophic equivalent. While the details of the geostrophic momentum formulation differ from the full shallow water development, all the essential differences between the latter and quasi-geostrophic theory are retained in the approximate set used here. Before developing the set of conservation statements, it should be mentioned that much of the present development parallels the work of Hayashi and Young (1987) (hereafter HY) on a one- 
layer, reduced gravity, two-front model on an equatorial $\beta$-plane.

\subsection{Conservation Statements}

From the full nonlinear, geostrophic momentum equations (i.e. before expanding in a basic state and a perturbation), (3.5) and (3.6), one can develop conservation statements for potential vorticity in the upper layer

$$
\frac{D q_{1}}{D t}=0
$$

where

$$
q_{1}=\frac{1+\zeta_{1_{x x}}+\zeta_{1_{y y}}-\zeta_{1_{x y}}^{2}+\zeta_{1_{x x}} \zeta_{1_{y y}}}{h_{1}}
$$

and in the lower layer

$$
\frac{D q_{2}}{D t}=0
$$

where

$$
q_{2}=\frac{1+\zeta_{T_{x x}}+\zeta_{T_{y y}}-\zeta_{T_{x y}}^{2}+\zeta_{T_{x x}} \zeta_{T_{y y}}}{h_{2}}
$$

(See Appendix A for details.) Again, $D / D t$ is given by (3.3) and contains the full advecting velocities. These definitions of potential vorticity are identifiable with the traditional shallow water forms. One difference is that the advected relative vorticity has been replaced with its geostrophic value, consistent with the derivation of the momentum equations in the previous chapter. The extra terms in the numerators of (4.1b) and (4.2b) contribute an ageostrophic component to the potential vorticity.

Similarly, an expression for the conservation of energy is

$$
\frac{\partial E}{\partial t}=0
$$

where

$$
E=\frac{1}{2} \int\left[h_{1}\left(\zeta_{1_{x}}^{2}+\zeta_{1_{y}}^{2}\right)+h_{2}\left(\zeta_{T_{x}}^{2}+\zeta_{T_{y}}^{2}\right)+h_{1}^{2}\right] d a
$$


The integral is defined over the whole domain of the fluid in the $x$-direction and over one wavelength in the $y$-direction. Again note the geostrophic form of the kinetic energies. Finally, an expression for absolute $y$-momentum is

$$
\frac{\partial M}{\partial t}=0
$$

where

$$
M=\int\left[h_{1}\left(\zeta_{1_{x}}+x\right)+h_{2}\left(\zeta_{T_{x}}+x\right)\right] d a .
$$

The terms in the integrand of (4.4b) proportional to $x$ arise because the system is in a rotating reference frame and they represent the momentum due to the Coriolis force. The Coriolis terms are absent in the definition of energy (4.3b) because the force acts in a direction perpendicular to the motion and thus does not contribute to the work done on a water parcel.

The expressions for $q_{1}, q_{2}, E$ and $M$ can be expanded into a basic state and a perturbation as described in the previous chapter. The potential vorticity becomes

$$
q_{1}=Q_{1}(x)+q_{1}^{\prime}(x, y, t)
$$

where

$$
Q_{1}(x)=\frac{1+\bar{v}_{x}}{\overline{h_{1}}}
$$

represents the basic state and

$$
q_{1}^{\prime}(x, y, t)=\frac{\zeta_{1_{x x}}+\left(1+\bar{v}_{x}\right) \zeta_{1_{y y}}-Q_{1}\left(\zeta_{1}-\zeta_{T}\right)}{\overline{h_{1}}}
$$

is the perturbation potential vorticity. $Q_{1}$ has the same form as in the shallow water equations while $q_{1}^{\prime}$ has the additional term $\bar{v}_{x} \zeta_{1 y y} / \overline{h_{1}}$. For the lower layer

$$
q_{2}=Q_{2}(x)+q_{2}^{\prime}(x, y, t)
$$

where

$$
Q_{2}(x)=\frac{1}{\overline{h_{2}}}
$$


and

$$
q_{2}^{\prime}(x, y, t)=\frac{\zeta_{T_{x x}}+\zeta_{T_{y y}}-Q_{2} \zeta_{2}}{\overline{h_{2}}} .
$$

Since there is no basic state flow in the lower layer, the basic state potential vorticity there is governed solely by the change in layer depth. The linearized forms of (4.1a) and (4.2a) for the perturbation potential vorticities are

$$
\begin{gathered}
q_{1_{t}}+\bar{v} q_{1_{y}}+u_{1} Q_{1_{x}}=0 \\
q_{2_{t}}+u_{2} Q_{2_{z}}=0
\end{gathered}
$$

where primes have been dropped.

The expansion of the energy conservation statement is slightly more complicated than that of potential vorticity due to the integration over the domain of the fluid. The undisturbed upper layer containing the mean current will occupy the area from $x_{f}$ to $-\infty$ in the cross-front direction (see Fig. 3.1). This can be denoted by $\int_{-\infty}^{x_{f}}() d A$ where the capital $\mathbf{A}$ represents the undisturbed area of the upper layer. A disturbance in the fluid will move the front to a position $x_{f}+\epsilon$ where $\epsilon \ll 1$ for small amplitude, linear theory. This extra area now occupied by the disturbed upper layer can be denoted by $\int_{x_{f}}^{x_{f}+\epsilon}() d a^{\prime}$. Note that this area will be an $0(\epsilon)$ quantity. In other words, the area integral is expanded as

$$
\int_{-\infty}^{x_{f}+\epsilon}(\quad) d a=\int_{-\infty}^{x_{f}}(\quad) d A+\int_{x_{f}}^{x_{f}+\epsilon}(\quad) d a^{\prime} .
$$

The expansion of $E$ can now proceed paying attention to the expansion of the fluid area at the same time.

The full energy (4.3b) can be written as

$$
E=E_{0}+E_{1}+E_{2}+\text { higher order terms, }
$$

where

$$
E_{0}=\frac{1}{2} \int\left(\overline{h_{1}} \bar{v}^{2}+{\overline{h_{1}}}^{2}\right) d A
$$


is the "basic state" energy,

$$
\begin{gathered}
E_{1}=\int\left[\overline{h_{1}} \bar{v} \zeta_{1_{z}}-\frac{1}{2} \bar{v}^{2} \zeta_{2}-\overline{h_{1}} \zeta_{2}\right] d A \\
+\quad \frac{1}{2} \int \bar{v}^{2}\left(\overline{h_{1}}-\zeta_{2}\right) d a
\end{gathered}
$$

is the "mean" energy (really the energy associated with the wave-mean flow interaction) and

$$
E_{2}=\frac{1}{2} \int\left[\overline{h_{1}}\left(\zeta_{1_{x}}^{2}+\zeta_{1_{y}}^{2}\right)-2 \bar{v} \zeta_{1_{x}} \zeta_{2}+\overline{h_{2}}\left(\zeta_{T_{x}}^{2}+\zeta_{T_{y}}^{2}\right)+\zeta_{2}^{2}\right] d A
$$

is the "wave" energy where primes have been dropped from perturbation quantities. Note that both $E_{1}$ and $E_{2}$ have $0\left(\epsilon^{2}\right)$ energy contributions. The $O\left(\epsilon^{2}\right)$ terms in $E_{1}$ can arise from both the second integral [because it spans an area $0(\epsilon)$ wide] and from the $0\left(\epsilon^{2}\right)$ parts of $\zeta_{1}$ and $\zeta_{2}$ in the first integral. Note that in the quasi-geostrophic limit, the second integral in (4.9c) vanishes (because the front can not surface) so the only $0\left(\epsilon^{2}\right)$ contributions to $E_{1}$ must come from the first integral.

The definition of $E_{2}$ contains the kinetic energy (in its geostrophic form) of the two layers and the potential energy due to the displacement of the interface. In addition, there is another term $-\bar{v} \zeta_{1_{x}} \zeta_{2}$ which is not positive definite. This term represents the correlation between geostrophic alongfront perturbation velocity $\left(\zeta_{1_{x}}=v_{1_{\theta}}\right)$ and perturbation interface displacement. For this term to be negative the correlation must be such that the disturbance increases the total upper layer thickness where it decreases the total alongfront speed. This pattern is illustrated in Fig. 4.1. This term is not present in the definition of wave energy in quasi-geostrophic theory because deviations of the interface from its basic state constant value are assumed small. In the quasi-geostrophic case, the wave energy is always positive definite.

Since $E_{0}$ does not change with time (4.3a) can be written as

$$
\frac{\partial\left(E_{1}+E_{2}\right)}{\partial t}=0
$$




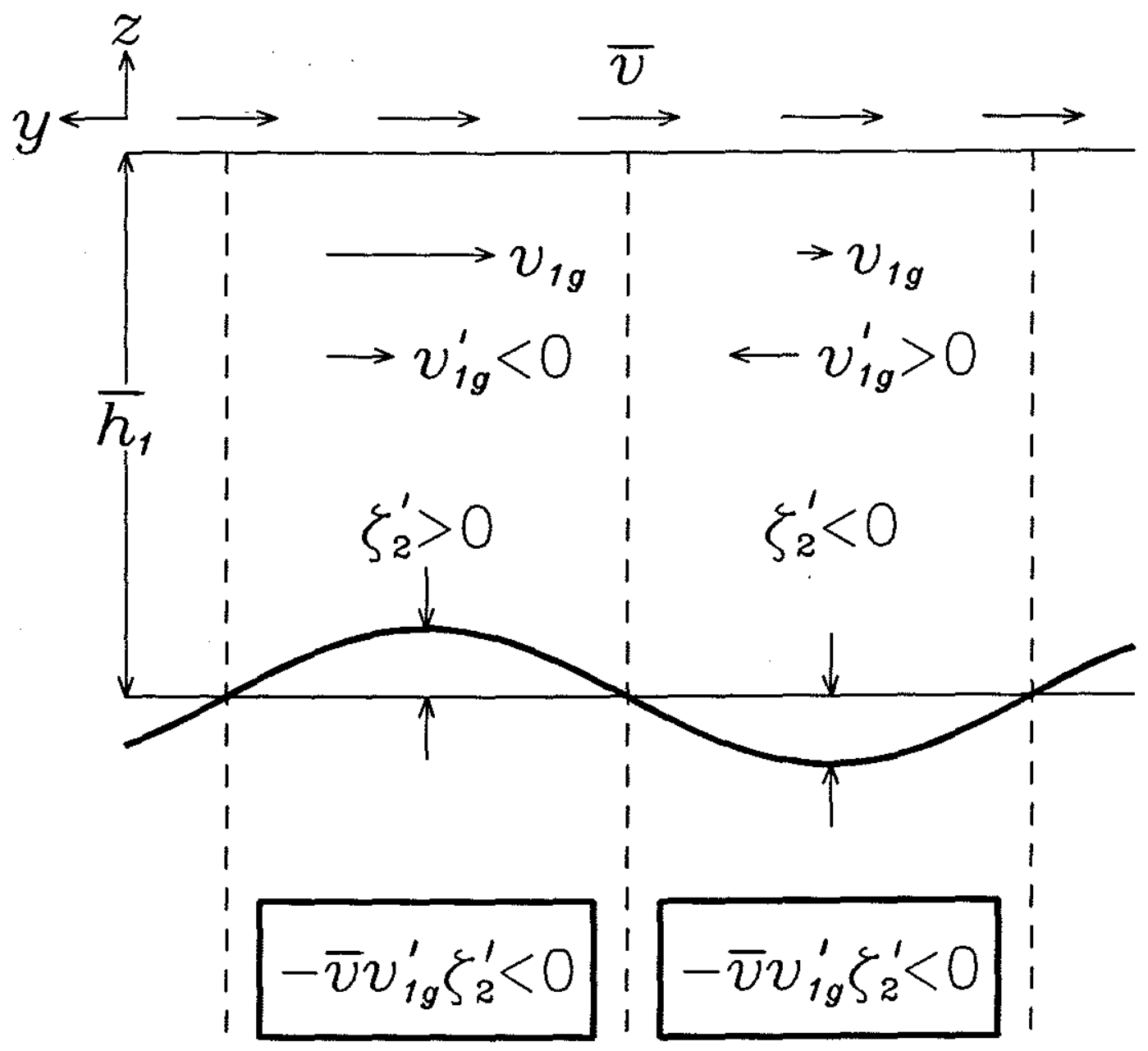

Figure 4.1: Correlation between geostrophic alongfront perturbation velocity $\left(v_{1_{q}}^{\prime}=S_{1_{z}}^{\prime}\right)$ and perturbation interface displacement $\left(\zeta_{2}^{\prime}\right)$ which results in a negative contribution (the boxed expression at the bottom of the figure) to the unstable wave energy $\left(E_{2}\right)$ defined in $(4.9 \mathrm{~d})$. The basic state flow $(\bar{v})$ is toward $-y$ in an unperturbed layer of depth $\overline{h_{1}}$. The sense of the correlation is such that the unstable wave increases the total alongfront geostrophic speed $\left(\left|v_{1}\right|=\left|\bar{v}+v_{1_{g}}^{\prime}\right|\right)$ where it decreases the total layer depth $\left(h_{1}=\overline{h_{1}}-\zeta_{2}^{\prime}\right)$ and vice versa. 
where terms of $0\left(\epsilon^{3}\right)$ and higher have been neglected. This statement is true for both stable and unstable disturbances. The following discussion is restricted to the case of an unstable disturbance. If this is integrated in time from some initial state when there is no unstable disturbance present then

$$
E_{1}+E_{2}=e=0 .
$$

The disturbance energy, $e$, is the energy in the disturbed system due to both the wave $\left(E_{2}\right)$ and the modification of the mean flow $\left(E_{1}\right)$. This allows for three limiting possibilities of energy distribution in the disturbance between the wave itself and the mean flow modifications associated with it:

$$
\begin{aligned}
& E_{1} \rightarrow-\infty \\
& E_{1}=0 \\
& E_{1} \rightarrow+\infty
\end{aligned}
$$

Note that the energies going to infinity are in the context of small-amplitude linear theory so that the true limiting values of the energies are scaled by $\epsilon$ where $\epsilon \ll 1$. The first case represents the familiar, traditional instability process. As the unstable wave grows exponentially its energy increases while that of the mean flow decreases. The second two cases arise because of the cross-term present in the definition of wave energy which allows for the possibility of "negative" energy. An unstable wave may grow in the system while its energy, as defined by (4.9d), and that of the mean flow, as defined by (4.9c), remain unchanged. HY term this "zero wave energy" instability. It will be demonstrated shortly that this case is relevant to a basic state with uniform potential vorticity. In the last case, as the unstable wave grows, its energy becomes increasingly negative while that of the mean increases. This has been termed "negative wave energy" instability by HY.

These statements about the transfer of energy within a system containing an unstable disturbance are certainly counterintuitive. In quasigeostrophic dynamics the basic state flow is always identified as a source of 
energy for the growing disturbance. Once the quasi-geostrophic approximation is abandoned these other forms of instability are possible. HY thus suggest that the idea of instabilities requiring a source of energy must be abandoned. These growing waves are possible because the total (mean plus wave) energy of the fluid is unaltered by the wave and the mean flow modifications associated with it. Note that even though the wave energy is not positive definite, which leads to counterintuitive ideas about energy transfer within the system, there still remain positive definite quantities which must increase with time for an unstable wave. One such quantity is the potential enstrophy (potential vorticity squared) which is positive definite and will increase with time as the unstable disturbance fluxes perturbation potential vorticity down the basic state potential vorticity gradient.

The idea of negative energy instability is not new and is quite familiar in plasma physics (see Cairns, 1979 for a reference list). Cairns (1979) has shown that for nonrotating stratified shear flows with step function velocity and density profiles, stable waves with negative energy can exist in the sense that exciting them lowers the total energy of the system. Specifically, Cairns (1979) shows that classic Kelvin-Helmholtz instability can occur because of the coexistence of positive and negative energy waves. Marinone and Ripa (1984) have studied large-scale instabilities on an equatorial Gaussian jet in a one-layer, reduced gravity model which have negative energy. Zero energy instabilities have been studied by HY and also arise, though not commented on explicitly, in the work of Griffiths et al. (1982) and Killworth et al. (1984).

In the classic frontal studies of Orlanski $(1968,1969)$ only the positive definite wave energy

$$
E_{2}^{+}=\frac{1}{2} \int\left[\overline{h_{1}}\left(\zeta_{1_{z}}^{2}+\zeta_{1_{y}}^{2}\right)+\overline{h_{2}}\left(\zeta_{T_{z}}^{2}+\zeta_{T_{y}}^{2}\right)+\zeta_{2}^{2}\right] d A
$$

was considered in the energy balance requiring the definition of an "interaction kinetic energy" which obscured the interpretation of the energy 
transfers within the system as outlined here and in HY. The recent work on frontal models of Griffiths et al. (1982), Killworth and Stern (1982), Killworth (1983) and Killworth et al. (1984) did not thoroughly address the energetics of the unstable waves and, as in Orlanski's work, only concentrated on the positive definite part of the wave energy (4.11).

In a significant contribution to the understanding of the instability process in these counterintuitive cases, HY suggested that the unstable waves can be thought of as roughly a linear combination of resonating shear modes each of which would be stable if the other were not present. The two resonating waves must have opposite signed disturbance (wave plus mean) energies so that the unstable mode has zero disturbance energy. The instability process is then an exchange of energy between the individual wave modes from the one with negative disturbance energy to the one with positive disturbance energy. In addition, destabilization by dissipation can be understood in the same context because it provides a sink for a wave with negative disturbance energy (Cairns, 1979). That is, the unstable mode grows as disturbance energy is removed from the mode with negative energy and lost to dissipation. These ideas will be commented on further in this chapter and confirmed in the numerical unstable wave solutions of chapter 5 .

The full $y$-momentum can be expanded in an analogous way to energy to obtain

$$
M=M_{0}+M_{1}+M_{2}+\text { higher order terms }
$$

Here

$$
M_{0}=\int\left[\overline{h_{1}}(\bar{v}+x)+\overline{h_{2}} x\right] d A
$$

is the basic state momentum,

$$
\begin{aligned}
M_{1}= & \int\left[\overline{h_{1}} \zeta_{1 x}-\bar{v} \zeta_{2}+\overline{h_{2}} \zeta_{T_{x}}\right] d A+ \\
& \int\left[\overline{h_{1}}(\bar{v}+x)+\overline{h_{2}} x-\bar{v} \zeta_{2}+\overline{h_{2}} \zeta_{T_{*}}\right] d a
\end{aligned}
$$


is the momentum of the mean flow and

$$
M_{2}=\int\left(\zeta_{T_{x}}-\zeta_{1_{x}}\right) \zeta_{2} d A
$$

is the wave momentum.

Expressions for the changes in wave energy (4.9d) and momentum (4.12d) can also be obtained from the linearized perturbation equations (3.8) and (3.9). The result (derived in Appendix A) for wave energy is

$$
\begin{aligned}
\frac{\partial E_{2}}{\partial t} & =-\int\left[\bar{h}_{1} \bar{v}_{x} u_{1} \zeta_{1_{x}}+\bar{v}^{2} u_{1} \zeta_{1_{x}}+\overline{h_{1}} \bar{v}\left(u_{1_{x}}+v_{1_{y}}\right) \zeta_{1_{x}}-\right. \\
\left.\bar{v} u_{1} \zeta_{2}-\bar{v}_{x} u_{1} \zeta_{2}\right] d A & \\
= & -\frac{\partial E_{1}}{\partial t} .
\end{aligned}
$$

The first two terms in the integrand can be recognized as the horizontal Reynolds stresses in their geostrophic momentum form. The third term represents the vertical Reynolds stress while the fourth term symbolizes the process of baroclinic instability. This latter process involves the cross-front flux of interface displacement or, in more physical terms, it is the crossfront movement of water in the two layers whose net effect is to flatten out the upwarped interface. As described in Pedlosky (1986) it is analogous to a slant-wise form of convection or a downgradient flux of heat in a continuously-stratified fluid. The baroclinic instability mechanism exchanges potential energy with the mean flow while the Reynolds stress terms exchange kinetic energy.

The final term in the integrand of (4.13) is not readily identifiable with a physical energy conversion process. It can, however, be related to the changes in time of the displacement of the surface front as detailed in Appendix A. This term can then be combined with the wave energy on the left-hand-side of (4.13). The final result is

$$
\frac{\partial}{\partial t}\left[E_{2}-\left.\frac{\bar{v}^{3}}{4} \int \epsilon^{2}\right|_{x=x_{f}} d y\right]=-\int\left[\bar{h}_{1} \bar{v}_{x} u_{1} \zeta_{1_{x}}+\bar{v}^{2} u_{1} \zeta_{1_{x}}+\right.
$$




$$
\left.\overline{h_{1}} \bar{v}\left(u_{1_{x}}+v_{1_{y}}\right) \zeta_{1_{x}}-\bar{v} u_{1} \zeta_{2}\right] d A .
$$

Since $\bar{v}<0$ the deflection of the front is a positive definite addition to the wave energy. Now the change in time of wave energy and the displacement of the surface front due to the wave can be attributed completely to the Reynolds stresses acting on the basic state flow and the baroclinic conversion of potential energy.

The conservation of wave energy can also be written in terms of the cross-front eddy flux of perturbation potential vorticity. The details are contained in Appendix A with the result

$$
\frac{\partial E_{2}}{\partial t}=\int{\overline{h_{1}}}^{2} \bar{v} u_{1} q_{1} d A+\frac{\partial}{\partial t} \int \overline{h_{1}} \bar{v} \zeta_{1_{x}} \zeta_{1_{y y}} d A
$$

where $E_{2}$ is given by (4.9d) and $q_{1}$ by (4.5c). The final term on the righthand-side arises solely due to the geostrophic momentum approximation. While $E_{2}$ is the exact geostrophic form of the wave energy, $q_{1}$ contains the geostrophic form of potential vorticity plus an extra term related to part of the ageostrophic potential vorticity. The integral in the final term in (4.15) can thus be related to part of the ageostrophic wave energy. To get an expression relating the time rate-of-change of wave energy to the flux of perturbation potential vorticity alone [without the extra term in (4.15)], the full form of the energy (geostrophic and ageostrophic) must be included in $E_{2}$. The resolution of this disparity between the shallow water form of the conservation of wave energy and the expression derived using the geostrophic momentum approximation is detailed in Appendix A. The final result is

$$
\frac{\partial E_{2}^{\star}}{\partial t}=\int{\overline{h_{1}}}^{2} \bar{v} u_{1} q_{1} d A,
$$

where the star $(\star)$ denotes the full (geostrophic plus ageostrophic) wave energy defined by

$$
E_{2}^{\star}=\frac{1}{2} \int\left[\overline{h_{1}}\left(u_{1}^{2}+v_{1}^{2}\right)-2 \bar{v} v_{1} \zeta_{2}+\overline{h_{2}}\left(u_{2}^{2}+v_{2}^{2}\right)+\zeta_{2}^{2}\right] d A
$$


and $q_{1}$ remains given by (4.5c). The energy equation (4.13) can also be modified to express the conservation of total energy by including the Reynolds stresses due to the ageostrophic part of the velocity field.

The flux of perturbation potential vorticity in (4.16) can be related to the dispersion of particles within a basic state potential vorticity gradient. Using (4.7) and substituting

$$
u_{1}=\left(\frac{\partial}{\partial t}+\bar{v} \frac{\partial}{\partial y}\right) \eta_{1},
$$

where $\eta_{1}$ is the displacement of particles in the upper layer, the conservation of potential vorticity can be written as

$$
\left(\frac{\partial}{\partial t}+\bar{v} \frac{\partial}{\partial y}\right)\left(q_{1}+\eta_{1} Q_{1_{x}}\right)=0 .
$$

If this expression is integrated with $q_{1}$ assumed initially to be zero, then

$$
q_{1}=-\eta_{1} Q_{1_{\mathbf{x}}}
$$

Substituting (4.17) and (4.19) into (4.16) one obtains

$$
\frac{\partial E_{2}^{\star}}{\partial t}=-\frac{\partial}{\partial t} \int{\bar{h}_{1}^{2}}^{2} Q_{1_{x}}\left(\eta_{1}^{2} / 2\right) d A .
$$

Clearly if the basic state potential vorticity is uniform $\left(Q_{1_{x}}=0\right)$ then $E_{2}^{\star}$ will not change with time. Integrating (4.20) with respect to time gives $E_{2}^{\star}$ equal to a constant and if the initial state contains no disturbance then this constant must be identically zero. In quasi-geostrophic theory a uniform potential vorticity basic state does not satisfy the necessary conditions for instability so $E_{2}^{\star}$ will always be zero which is in agreement with (4.20). By abandoning the restrictions required by quasi-geostrophic theory it has been shown previously in this section that an unstable mode may exist if the total disturbance energy $\left(E_{1}^{\star}+E_{2}^{\star}\right)$ is equal to zero. The existence of this "zero wave energy" mode of instability has been confirmed by Griffiths 
et al.(1982) and by HY and will be shown to exist for the two-layer coastal upwelling front model in chapter 6 . From (4.20) it is also clear that the sign of $E_{2}^{\star}$ can be determined from the sign of $Q_{1_{x}}$. For $Q_{1_{x}}<0$ the wave energy will be negative as in the study of Marinone and Ripa (1984) and it can be shown that the one-layer unstable mode of Killworth (1983) is also of this type. For $Q_{1_{x}}>0$ the traditional positive wave energy unstable mode is recovered.

Conservation of wave $y$-momentum derived from the linearized perturbation equations (3.8) and (3.9) (see Appendix A for details) is

$$
\begin{aligned}
\frac{\partial M_{2}}{\partial t}= & \int\left[{\overline{h_{1}}}^{2} u_{1} q_{1}+{\overline{h_{2}}}^{2} u_{2} q_{2}\right] d A+ \\
& \frac{\partial}{\partial t} \int\left[\overline{h_{1}} \zeta_{1_{x}} \zeta_{1_{y y}}+\overline{h_{2}} \zeta_{T_{z}} \zeta_{T_{y y}}\right] d A,
\end{aligned}
$$

where the extra terms arise in an analogous manner to those in (4.15). They can be removed by considering the full $y$-momentum with the result

$$
\frac{\partial M_{2}^{\star}}{\partial t}=\int\left[{\overline{h_{1}}}^{2} u_{1} q_{1}+{\overline{h_{2}}}^{2} u_{2} q_{2}\right] d A
$$

where the full (geostrophic plus ageostrophic) wave momentum is defined by

$$
M_{2}^{\star}=\int\left(v_{2}-v_{1}\right) \zeta_{2} d A .
$$

Using (4.17), (4.19) and similar expressions for the lower layer the conservation of wave $y$-momentum can be related to the flux of perturbation potential vorticity in both layers

$$
\frac{\partial M_{2}^{\star}}{\partial t}=-\frac{\partial}{\partial t} \int\left[{\overline{h_{1}}}^{2} Q_{1_{x}}\left(\eta_{1}^{2} / 2\right)+{\overline{h_{2}}}^{2} Q_{2_{x}}\left(\eta_{2}^{2} / 2\right)\right] d A .
$$

It is important to note that an equation like (4.16) relating the change of wave energy to the flux of perturbation potential vorticity cannot be written for the positive definite quantity $E_{2}^{+}$given by (4.11). Consequently, the time rate-of-change of $E_{2}^{+}$cannot be related to the dispersion of particles 
in an unstable wave like in (4.20). This lack of connection between $E_{2}^{+}$ and particle displacements (a property of unstable waves whose increasing dispersion with time is a fundamental diagnostic of instability) rules out $E_{2}^{+}$as the correct definition of wave energy. Instead, $E_{2}$ (or $E_{2}^{\star}$ ) is a more useful measure of energy in a growing wave.

\subsection{Stability Theorem}

Using the conservation of energy and $y$-momentum statements, a general stability theorem can be derived. The method employed here is an extension of that of Ripa (1983) who developed a theorem for a one-layer, reduced gravity model on an equatorial $\beta$-plane or sphere. The definitions of $E_{2}^{\star}$ and $M_{2}^{\star}$ can be combined using an arbitrary constant $\gamma$

$$
\begin{gathered}
E_{2}^{\star}-\gamma M_{2}^{\star}=\frac{1}{2} \int\left[\overline{h_{1}}\left(u_{1}^{2}+v_{1}^{2}\right)+\overline{h_{2}}\left(u_{2}^{2}+v_{2}^{2}\right)-2(\bar{v}-\gamma) v_{1} \zeta_{2}+\right. \\
\left.\zeta_{2}^{2}-2 \gamma v_{2} \zeta_{2}\right] d A .
\end{gathered}
$$

The integrand can be rewritten by completing the square of the terms involving $v_{1}$ and $v_{2}$ with the result

$$
\begin{aligned}
E_{2}^{\star}-\gamma M_{2}^{\star}= & \frac{1}{2} \int\left\{\left[\left(\overline{h_{1}}\right)^{1 / 2} v_{1}-\frac{(\bar{v}-\gamma) \zeta_{2}}{\left(\overline{h_{1}}\right)^{1 / 2}}\right]^{2}+\left[\left(\overline{h_{2}}\right)^{1 / 2} v_{2}-\frac{\gamma \zeta_{2}}{\left(\overline{h_{2}}\right)^{1 / 2}}\right]^{2}+\right. \\
& \left.\overline{h_{1}} u_{1}^{2}+\overline{h_{2}} u_{2}^{2}+\zeta_{2}^{2}-\frac{(\bar{v}-\gamma)^{2} \zeta_{2}^{2}}{\overline{h_{1}}}-\frac{\zeta_{2}^{2} \gamma^{2}}{\overline{h_{2}}}\right\} d A .
\end{aligned}
$$

If the last three groups of terms are combined as

$$
\zeta_{2}^{2}\left[1-\frac{(\bar{v}-\gamma)^{2}}{\overline{h_{1}}}-\frac{\gamma^{2}}{\overline{h_{2}}}\right],
$$

the entire integrand will be positive definite if

$$
(\bar{v}-\gamma)^{2}+\gamma^{2} \mu \leq \overline{h_{1}}
$$


for all $x$ where $\mu=\overline{h_{1}} / \overline{h_{2}}$ is the depth ratio. By setting the arbitrary constant $\gamma$ equal to zero, (4.26) is satisfied if the magnitude of the upper layer mean flow is everywhere less than the internal gravity wave phase speed $\left(\left(\overline{h_{1}}\right)^{1 / 2}\right.$ nondimensionally). This type of flow is known as "subcritical". With a deep lower layer $(\mu \ll 1)$ allowing $\gamma$ to be nonzero allows $\bar{v}$ to be supercritical somewhere in the flow and still satisfy (4.26). The inclusion of a finite depth lower layer makes (4.26) very difficult to satisfy for the frontal flows of interest here.

The conservation statements for $E_{2}^{\star}$ and $M_{2}^{\star},(4.20)$ and (4.23), can also be combined with the use of the same arbitrary constant $\gamma$ to yield

$$
\frac{\partial\left(E_{2}^{\star}-\gamma M_{2}^{\star}\right)}{\partial t}=-\frac{\partial}{\partial t} \int\left[{\overline{h_{1}}}^{2}(\bar{v}-\gamma) Q_{1_{x}}\left(\eta_{1}^{2} / 2\right)-{\overline{h_{2}}}^{2} \gamma Q_{2_{x}}\left(\eta_{2}^{2} / 2\right)\right] d A .
$$

Integrating with respect to time, this becomes

$$
\underbrace{\left(E_{2}^{\star}-\gamma M_{2}^{\star}\right)}+\int[\underbrace{{\overline{h_{1}}}^{2}(\bar{v}-\gamma) Q_{1_{x}}\left(\eta_{1}^{2} / 2\right)} \underbrace{-{\overline{h_{2}}}^{2} \gamma Q_{2_{x}}\left(\eta_{2}^{2} / 2\right)}] d A=\text { constant }
$$

where the basic state potential vorticity gradients are defined by $(4.5 \mathrm{~b})$ and (4.6b). If each of the three groups of terms in this expression is positive then no increase in wave properties (e.g. energy, particle dispersion) with time is allowed. A mixture of positive and negative terms can allow growth of the wave amplitude while still satisfying this expression. The first group will be positive if (4.26) is satisfied. That is, even though $E_{2}^{*}$ contains the term $-\bar{v} v_{1} \zeta_{2}$ which may make the wave energy negative and $\gamma$ may be chosen so that $-\gamma M_{2}^{\star}$ will be negative, their linear combination will be positive definite if condition (4.26) holds. Requiring the final two groups of terms to be positive leads to the statement of a general stability theorem, viz.:

If there exists any value of $\gamma$ such that

$$
(\bar{v}-\gamma)^{2}+\gamma^{2} \mu \leq \overline{h_{1}},
$$




$$
\begin{aligned}
(\bar{v}-\gamma) Q_{1_{x}} & \geq 0 \quad \text { and } \\
\gamma Q_{2_{x}} & \leq 0
\end{aligned}
$$

for all $x$ then the flow is stable to infinitesimal perturbations.

These conditions are sufficient to insure stability and (4.27) is essentially a two-layer version of Ripa's (1983) theorem. The extension to an arbitrary number of layers requires the addition of statements like $(4.27 \mathrm{c})$ for each layer. The stability criteria (4.27) are also similar to those of Long (1987) for continuously stratified, rotating flows. He finds a restriction on the vertical scale of the disturbance to which the flow will be stable. This condition is analogous to (4.27a) if the scale of the disturbance is identified with the vertical wavelength of a long internal wave in a continuously stratified fluid. In the present study, the vertical scale of the disturbances is set by the layer depths. In either case, the flow will be stable if the mean flow is everywhere less than the internal gravity wave phase speed [and $(4.27 \mathrm{~b})$ and $(4.27 \mathrm{c})$ are satisfied].

Quasi-geostrophic flows are generally weak (i.e. slow relative to the internal gravity wave speed) so they easily satisfy (4.27a). The remaining conditions $(4.27 \mathrm{~b})$ and $(4.27 \mathrm{c})$ are just the familiar requirement that a change in the sign of the basic state potential vorticity gradient exist in order to get instability. Specifically, (4.27b) is just Fjørtoft's (1950) theorem (with $\gamma$ equal to the value of $\bar{v}$ at the inflection point) or equivalent to that of Kuo (1949) and Rayleigh (1880) (with $\gamma$ outside the range of $\bar{v}$ ). The additional constraint (4.27c) allows the possibility of instability even if $(4.27 \mathrm{~b})$ is satisfied by making the change in sign of the basic state potential vorticity gradient occur between layers.

For the strong flows associated with frontal regions, unstable waves may still exist even if there is no change in the sign of the basic state potential vorticity gradient. This can occur if the first condition (4.27a) is violated as discussed above in association with (4.26). 
The stability theorem (4.27) can now be applied to the coastal upwelling model of interest here by making an explicit choice for $\overline{h_{1}}(x)$ and via geostrophy (3.7) fixing the basic state upper layer flow. The choice for the interface profile is a family of exponentials given by

$$
\overline{h_{1}}(x)= \begin{cases}1-\exp \left[\alpha\left(x-x_{f}\right)\right] & x \leq x_{f} \\ 0 & x \leq x_{f}\end{cases}
$$

so that

$$
\bar{v}(x)=-\alpha \exp \left[\alpha\left(x-x_{f}\right)\right] \text { for } x \leq x_{f} .
$$

A uniform potential vorticity basic state will have $\alpha=1.0$. For $\alpha<1.0$ the interface is less steeply sloping than the uniform potential vorticity front (e-folding length greater than 1.0 which in dimensional units is the internal Rossby radius of deformation) and for $\alpha>1.0$ the front is more steeply sloping (e-folding length less than $R$ ).

For a uniform potential vorticity front $(\alpha=1.0)(4.27 \mathrm{~b})$ is automatically satisfied. For a deep lower layer, $\mu \ll 1$ and (4.27c) is automatically satisfied because the lower layer perturbation velocities vanish. For quasi-geostrophic flow, satisfying $(4.27 \mathrm{~b})$ would alone be sufficient to insure stability. However, for these ageostrophic models, (4.27a) must also be satisfied. With (4.28) this condition becomes

$$
\left[\exp \left(x-x_{f}\right)+\gamma\right]^{2} \leq 1-\exp \left(x-x_{f}\right)
$$

which is satisfied for all $x$ if $\gamma=-1$. A one-layer, reduced gravity model with uniform basic state potential vorticity is thus stable to infinitesimal perturbations of all scales. This is the same result as that of Paldor (1983) who used a Rayleigh integral technique applied directly to the governing equations.

Again, for a model with a deep lower layer, the stability of nonuniform potential vorticity $(\alpha \neq 1.0)$ flows can be determined by examining (4.27a) and (4.27b). Substituting (4.28) into (4.27a) yields

$$
\left\{\alpha \exp \left[\alpha\left(x-x_{f}\right)\right]+\gamma\right\}^{2} \leq 1-\exp \left[\alpha\left(x-x_{f}\right)\right] .
$$


Examination of this inequality shows that it will only hold for $\gamma=-\alpha$ and $\alpha<1$. With $\gamma=-\alpha(4.27 \mathrm{~b})$ becomes

$$
\left\{\exp \left[\alpha\left(x-x_{f}\right)\right]-1\right\} Q_{1_{z}} \leq 0 .
$$

For $\alpha<1$, the upper layer basic state potential vorticity gradient will be greater than zero and this expression is satisfied for all $x$. Conversely, for $\alpha>1$, the upper layer basic state potential vorticity gradient will be less than zero and this inequality will not hold for any $x \neq x_{f}$. The conclusion from this is that for $\alpha<1$ or "shallow" interface profiles a one-layer reduced gravity model is stable. This can also be rationalized by realizing that $\alpha \ll 1$ is the correct limit to recover quasi-geostrophic flow. For $\alpha>1$, or "steep" interface profiles, the one-layer front may be unstable. This dependence on $\alpha$ of the stability of the front is in agreement with the results of Killworth (1983) who analytically solved the governing equations in a long wave limit by a rather complicated boundary layer analysis.

All the above discussion is relevant to a model with a deep lower layer. Inspection of (4.27) for a finite lower layer depth shows that no choice of $\gamma$ will satisfy all three constraints even for a uniform potential vorticity basic state. With this basic state and a flat bottom, unstable modes may exist because both (4.27a) and (4.27c) are violated. For a basic state with uniform potential vorticity in the upper layer the bottom topography may be chosen to satisfy $(4.27 \mathrm{c})$. However, the inequality $(4.27 \mathrm{a})$ will still not hold for all $x$ giving the possibility for unstable modes. Note that standard quasi-geostrophic stability criteria would insure the stability of this flow. This clearly illustrates the danger in applying stability criteria derived from quasi-geostrophic theory to frontal configurations.

In summary, the stability theorem derived here (4.27) suggests that the coastal upwelling front (since it exists in shallow water) may be unstable no matter what the basic state flow configuration is. In violating sufficient conditions for stability the flow satisfies necessary conditions for instability. 
It is still essential to verify that unstable waves do exist on the coastal upwelling front and this is done in the next chapter by solving numerically the governing equations (3.8) and (3.9). 


\section{Chapter 5}

\section{Results}

\subsection{Introduction}

The results of chapter 4 suggest that unstable disturbances exist on coastal upwelling fronts. Violation of the sufficient stability conditions (4.27) does not, however, guarantee the presence of an unstable wave. The purpose of this chapter is to prove that growing disturbances do in fact exist on a coastal upwelling front by solving the governing equations (3.8) and (3.9) numerically. First, the solution technique will be described including a detailed look at the various boundary conditions required. Next, results will be presented for a model with an inviscid basic state flow and a flat bottom. The particular case of a front with uniform potential vorticity located far from a coastal barrier can be compared to the results of Killworth et al. (1984). The effects on the properties of the unstable waves produced by varying the total depth, the distance to the coastal wall and the shape of the interface profile will be discussed. For one standard case, the energy transfers in the system will be analyzed in detail and the conservation statements of chapter 4 will be confirmed. The effect of a strongly sloping bottom will be presented in section 5.4. Finally, linearized bottom friction is included and its influence on the unstable waves discussed. 


\subsection{Solution Technique}

Instability calculations generally follow one of two traditional methods. The evolution on an unstable disturbance can be found by solving the governing equations as an initial value problem. This usually requires various transform techniques (Laplace, Fourier) as part of the solution and can be quite complicated. An alternative approach is to recognize that since the coefficients of (3.8) and (3.9) depend on $x$ only, solutions may be sought which are periodic in $y$. The normal mode form appropriate to this study using perturbation sea surface elevation as an example is

$$
\zeta_{1}(x, y, t)=\Re\left[\hat{\zeta_{1}}(x) \exp i(l y-\sigma t)\right] .
$$

Here $l$ is the (real) alongfront wavenumber and both $\sigma$, the frequency, and $\hat{\zeta_{1}}(x)$, the cross-front amplitude function, are complex. $\Re$ denotes the real part of the expression in square brackets. Since $\sigma=\sigma_{r}+i \sigma_{i}$ is complex, unstable solutions with $\sigma_{i}>0$ will grow exponentially with time. This can be seen explicitly by splitting $\sigma$ into its real and imaginary parts and substituting into (5.1) to get

$$
\zeta_{1}(x, y, t)=\Re\left[\hat{\zeta_{1}}(x) \exp i\left(l y-\sigma_{r} t\right)\right] \exp \left(\sigma_{i} t\right)
$$

With $\sigma_{i}>0$ the initial disturbance will grow until the nonlinear terms neglected in the linearized equations (3.8) and (3.9) become large. When this occurs the linear theory presented here is no longer adequate to describe the evolution of the unstable waves. Nevertheless, linear theory will successfully describe the initial instability process and provide details of the small amplitude behavior of the unstable waves.

The solution technique proceeds as follows. Substituting (5.1) and similar expressions for $\zeta_{T}$ and the layer velocities into (3.8) and (3.9) yields

$$
(\sigma-l \bar{v}) l \zeta_{1}+v_{1}=\zeta_{1_{x}}
$$




$$
\begin{gathered}
-i(\sigma-l \bar{v}) \zeta_{1_{x}}+\left(1+\bar{v}_{x}\right) u_{1}=-i l \zeta_{1} \\
-i(\sigma-l \bar{v})\left(\zeta_{1}-\zeta_{T}\right)+\left(u_{1} \overline{h_{1}}\right)_{x}+i l v_{1} \overline{h_{1}}=0
\end{gathered}
$$

for the upper layer and

$$
\begin{gathered}
\sigma l \zeta_{T}+v_{2}=\zeta_{T_{x}} \\
-i \sigma \zeta_{T_{x}}+u_{2}=-i l \zeta_{T} \\
-i \sigma \zeta_{T}+\left(u_{2} \overline{h_{2}}\right)_{x}+i l v_{2} \overline{h_{2}}=0
\end{gathered}
$$

for the lower layer. (Note that the hats ^ have been dropped.) Next, the first two equations in (5.3) and (5.4) are used to solve for the layer velocities

$$
\begin{gathered}
u_{1}=\frac{i\left[(\sigma-l \bar{v}) \zeta_{1_{x}}-l \zeta_{1}\right]}{1+\bar{v}_{x}} \\
v_{1}=\zeta_{1_{x}}-(\sigma-l \bar{v}) l \zeta_{1} \\
u_{2}=i\left(\sigma \zeta_{T_{x}}-l \zeta_{T}\right) \\
v_{2}=\zeta_{T_{x}}-\sigma l \zeta_{T}
\end{gathered}
$$

These expressions can be substituted into the continuity equations (5.3c) and (5.4c) to get a single equation for each layer. The result for the upper layer is

$$
\begin{aligned}
\overline{h_{1}} \zeta_{1_{x x}}+\left[\bar{v}-\frac{\bar{v}_{x x} \overline{h_{1}}}{\left(1+\bar{v}_{x}\right)}\right] \zeta_{1_{x}}-\left(1+\overline{h_{1}} l^{2}\right)\left(1+\bar{v}_{x}\right) \zeta_{1}+\left(1+\bar{v}_{x}\right) \zeta_{T}= \\
\quad \frac{l \bar{v}}{\sigma}\left\{\overline{h_{1}} \zeta_{1_{x x}}+\left[\bar{v}-\frac{\bar{v}_{x x} \overline{h_{1}}}{\left(1+\bar{v}_{x}\right)}\right] \zeta_{1_{x}}-\right. \\
\\
{\left.\left[\left(1+\overline{h_{1}} l^{2}\right)\left(1+\bar{v}_{x}\right)-1+\frac{\bar{v}_{x x} \overline{h_{1}}}{\bar{v}\left(1+\bar{v}_{x}\right)}\right] \zeta_{1}+\left(1+\bar{v}_{x}\right) \zeta_{T}\right\} }
\end{aligned}
$$

The lower layer equation becomes

$$
\zeta_{T_{x x}}+\frac{\overline{h_{2 x}}}{\overline{h_{2}}} \zeta_{T_{x}}-\left(\frac{1}{\overline{h_{2}}}+l^{2}\right) \zeta_{T}+\frac{1}{\overline{h_{2}}} \zeta_{1}=\frac{l \overline{h_{2}} x}{\sigma \overline{h_{2}}} \zeta_{T}
$$


These two equations are coupled due to the appearance of $\zeta_{1}$ and $\zeta_{T}$ in each. The complex frequency $\sigma$ is the eigenvalue for this problem and only appears linearly in (5.7). If the geostrophic momentum approximation is not made the eigenvalue will appear nonlinearly in the shallow water equation equivalent of (5.7). The numerical solution technique to be described shortly is greatly simplified by having made the geostrophic momentum approximation.

Note that the upper layer equation (5.7a) is singular at several points within the domain of the fluid. Singularities exist where $\overline{h_{1}}=0$ (at the front, $x=x_{f}$ ), where the horizontal shear of the mean flow exactly balances the Coriolis parameter $\left(1+\bar{v}_{x}=0\right)$ and at a "critical layer" where the phase speed of the wave equals the mean flow speed $(\sigma=l \bar{v}$ or $c=\bar{v}$ with $c=\sigma / l$ being the wave phase speed). These singularities make the analytic solution of (5.7) [and the reduced gravity version of (5.7a)] very difficult. Progress can only be made using various approximations such as assuming long waves [Killworth (1983) for a reduced gravity shallow water equation model], nearly uniform potential vorticity [Kubokawa (1985) again for a one-layer model] or long waves and deep lower layers [Killworth et al. (1984) for a two-layer isolated front]. In all cases the mathematics required are quite complicated and involve careful matching of solutions across various boundary layers. In the two-layer case these analytic solution techniques fail when the bottom topography differs from a flat bottom.

Since little progress can be made analytically on the system (5.7) a numerical solution technique is desirable. It should be mentioned that numerical solution techniques are not infallible. Problems will arise when the growth rate of the unstable waves approaches zero. Nevertheless, the solution technique described next will yield useful results away from these points. The coupled layer equations can be cast in the form of an algebraic eigenvalue problem by splitting the cross-front domain into finite intervals and approximating the derivatives in (5.7) by finite differences. The prob- 
lem becomes, after multiplying by the eigenvalue $\sigma$,

$$
\underset{\approx}{A} \underset{\sim}{\eta}=\sigma \underset{\sim}{B} \underset{\sim}{\eta}
$$

where $\underset{\sim}{A}$ and $\underset{\approx}{B}$ are matrices containing the finite difference equivalents of the derivatives in (5.7) and $\underset{\sim}{\eta}$ is the column vector defined as

$$
\underset{\sim}{\eta}=\left(\begin{array}{c}
\zeta_{1}^{1} \\
\zeta_{1}^{2} \\
\cdot \\
\cdot \\
\zeta_{1}^{L-1} \\
\zeta_{1}^{L} \\
\hline \zeta_{T}^{1} \\
\zeta_{T}^{2} \\
\cdot \\
\cdot \\
\cdot \\
\zeta_{T}^{L-1} \\
\zeta_{T}^{L} \\
\zeta_{T}^{L+1} \\
\cdot \\
\cdot \\
\dot{\zeta_{T}^{N}-1} \\
\zeta_{T}^{N}
\end{array}\right)
$$

In the definition of $\eta$ the superscripts on $\zeta_{1}^{i}$ and $\zeta_{T}^{i}$ denote the values of these variables at the $i$ th grid point in $x$. The $x$-domain runs from $i=1$ at $x=-\infty$ (in the numerically truncated domain $i=1$ corresponds to a location several Rossby radii from the front), through $i=L$, which denotes the location of the surface front, to $i=N$ at the coastal barrier. Note that $\zeta_{1}$ only exists from $i=1$ to $i=L$ while $\zeta_{T}$ is defined at every point in the numerical domain. Since (5.8) contains both the coupled layer equations 
simultaneously, $\underset{\approx}{A}$ and $\underset{\approx}{B}$ are $(N+L) \times(N+L)$ in size. Another property of (5.8) is that $\underset{\approx}{A}$ and $\underset{\approx}{B}$ are real so that (5.8) is a real algebraic eigenvalue problem of general form. The eigenvalues $(\sigma)$ and eigenfunctions $\underset{\sim}{(})$ can be found numerically by using, for example, a QZ algorithm. The solutions presented here were found using the IMSL (International Math Subroutine Library) routine EIGZF.

In setting up the finite difference grid in the $x$-direction several choices must be made concerning the number of points, the size of the interval between grid points and how far away from the surface front the model will extend. The distance between grid points was chosen to be 0.06 Rossby radii $(R)$ so that for a front with uniform basic state potential vorticity 16 points would lie within an e-folding length of the surface front. The numerical domain was chosen to extend $4 R$ from the surface front. Reducing the interval between grid points to $0.04 R$ or increasing the distance of the edge of the numerical domain from the front resulted in less than $0.5 \%$ change in the eigenvalue $\sigma$. Results produced with a coarser grid or a smaller domain were generally not reliable. For a typical model configuration with the surface front lying $2 R$ from the coastal barrier, the number of points $(N)$ was 93 with $L=61$. This made $\underset{\sim}{A}$ and $\underset{\sim}{B} 154 \times 154$ and execution time on a DEC (Digital Equipment Corporation) MicroVAX II was approximately 18 CPU minutes.

The two second order, ordinary differential equations (5.7) require four boundary conditions to specify a unique solution. The development and numerical application of the necessary boundary conditions are detailed below. The difference in the form of the boundary conditions for both an isolated front over a flat bottom (Fig. 5.1a) and a front next to a coastal barrier with arbitrary bottom topography (Fig. 5.1b) will be discussed. Note that since the logical reference point in the cross-front direction for the isolated front (Fig. 5.1a) is the point where the front intersects the 

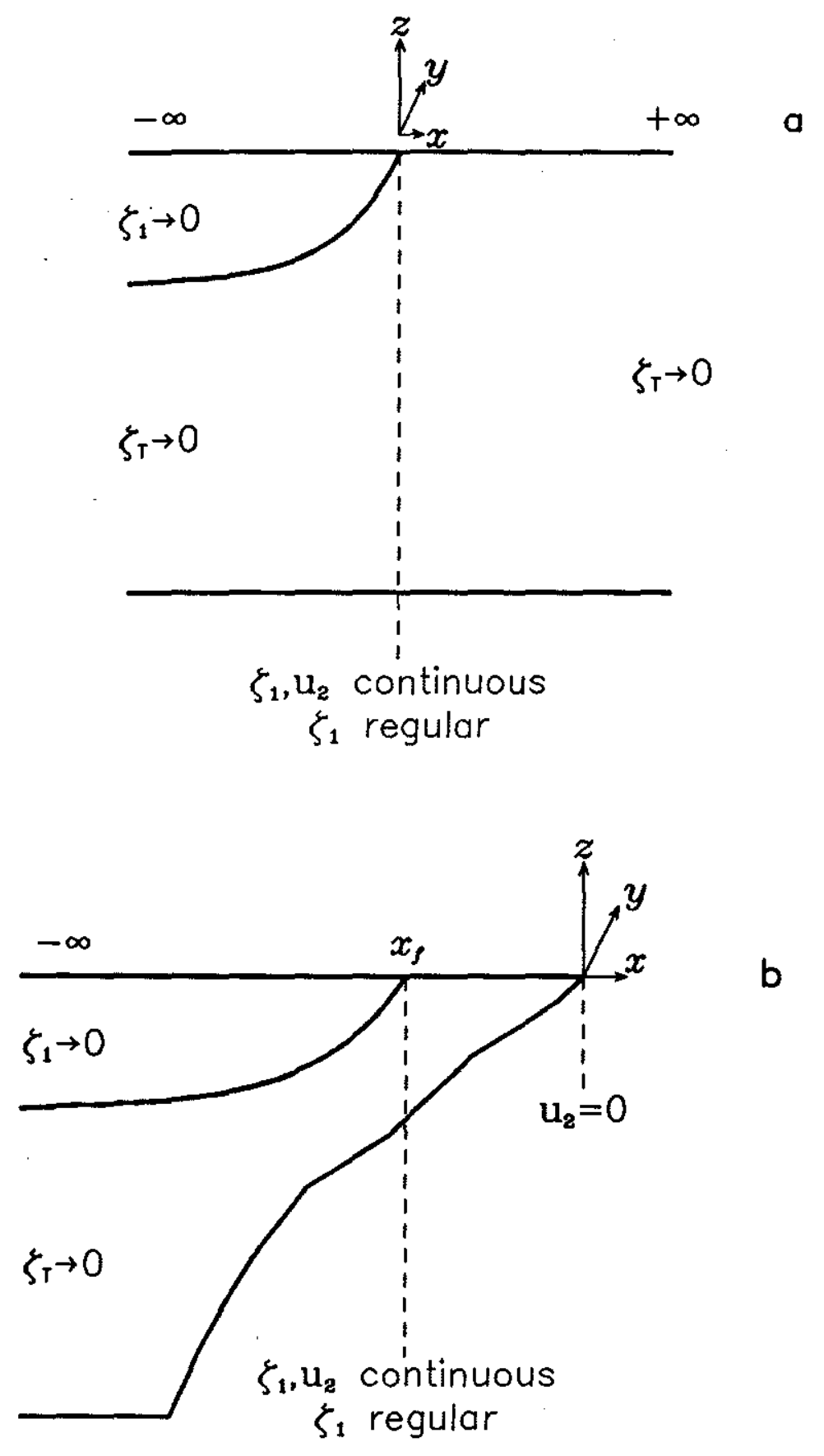

Figure 5.1: Pictorial representation of the boundary conditions for (a) an isolated front, where the origin of the coordinate system is located at the surface front, and (b) a front over arbitrary cross-shelf bottom topography next to a coastal barrier. 
surface, the origin of the coordinate system has been placed there. The solutions given below can be tailored to this case by setting $x_{f}=0$. One general requirement is that the solutions to (5.7) be frontally-trapped. Far from the surface front in the two-layer region of the $x$-domain $(x \rightarrow-\infty)$ the interface becomes flat. In addition, this region is assumed to have a flat bottom (Fig. 5.1). The governing equations (5.7) reduce to a coupled pair of constant coefficient ordinary differential equations which can be solved analytically. The solutions which satisfy the boundary conditions

$$
\zeta_{1}, \zeta_{T} \rightarrow 0 \quad \text { as } \quad x \rightarrow-\infty
$$

are

$$
\begin{gathered}
\zeta_{1}=c_{1} \exp \left[r_{1}\left(x-x_{f}\right)\right]+c_{2} \exp \left[r_{2}\left(x-x_{f}\right)\right] \\
\zeta_{T}=-\frac{H_{1}}{H_{2}} c_{1} \exp \left[r_{1}\left(x-x_{f}\right)\right]+c_{2} \exp \left[r_{2}\left(x-x_{f}\right)\right]
\end{gathered}
$$

where $c_{1}$ and $c_{2}$ are arbitrary constants, $H_{1}$ and $H_{2}$ are the constant layer depths (see Fig. 3.1),

$$
r_{1}=\left(l^{2}+\frac{H_{1}+H_{2}}{H_{1} H_{2}}\right)^{1 / 2}
$$

is an inverse decay scale containing the two-layer form of the internal Rossby radius of deformation and

$$
r_{2}=l
$$

is an inverse decay scale based on the alongfront wavenumber. The constants $c_{1}$ and $c_{2}$ can be eliminated by requiring continuity of $\zeta_{1}$ and $\zeta_{T}$ at the edge of the numerical domain $(i=1)$. Finally, the boundary conditions may be incorporated into the numerical scheme using standard finite difference techniques.

On the one-layer side of the front, one of two boundary conditions is required. For an isolated front far from any coastal barrier (Fig. 5.1a) the solution must satisfy

$$
\zeta_{T} \rightarrow 0 \quad \text { as } \quad x \rightarrow+\infty .
$$


In this case the simplest model geometry is one with a flat bottom and an analytic solution to $(5.7 \mathrm{~b})$ can be found which satisfies the above boundary condition. The solution for $x>0$ where, as noted above, the $x$-origin is now assumed to lie at the surface front $\left(x_{f}=0\right)$ is

$$
\zeta_{T}^{+}=c_{3} \exp (-\alpha x)
$$

where

$$
\alpha^{2}=l^{2}+\frac{\delta}{H}
$$

This solution can be used to match to the two-layer region at $x=0$ by requiring continuity of sea surface elevation and normal velocity between the lower $(x<0)$ layer and the single $(x>0)$ layer. Continuity of sea surface elevation

$$
\zeta_{1}^{-}=\zeta_{1}^{+}
$$

can be expanded in a Taylor series about the unperturbed frontal location $(x=0)$. Keeping only the first two terms in the expansion yields

$$
\left.\left.\left[\overline{\zeta_{1}^{-}}+\zeta_{1}^{-}\right]\right|_{x=0}+\epsilon \overline{\left[{\zeta_{x}}_{x}\right.}+\zeta_{1_{x}}^{-}\right]\left.\right|_{x=0}=\left.\zeta_{1}^{+}\right|_{x=0}+\left.\epsilon \zeta_{1_{x}}^{+}\right|_{x=0},
$$

where the overbar represents a mean quantity and $\epsilon$ is a small displacement of the front. Since $\overline{\zeta_{1}^{-}}=0$ at $x=0$ by definition and $\overline{\zeta_{1_{x}}}=\bar{v}$ this becomes to $0(\epsilon)$

$$
\zeta_{1}^{-}+\bar{v} \epsilon=\zeta_{1}^{+} \quad \text { at } \quad x=0 .
$$

At the perturbed frontal position $(x=\epsilon)$ the total upper layer depth vanishes

$$
h_{1}+\delta \zeta_{1}=0 \quad \text { at } \quad x=\epsilon .
$$

This can be expanded in a Taylor series to get an expression valid at the undisturbed frontal location

$$
\left.\left[(1-\delta) \overline{h_{1}}+\delta \overline{\zeta_{1}}+\zeta_{1}-\zeta_{T}\right]\right|_{x=0}+\left.\epsilon \frac{\partial}{\partial x}\left[(1-\delta) \overline{h_{1}}+\delta \overline{\zeta_{1}}+\zeta_{1}-\zeta_{T}\right]\right|_{x=0}=0
$$


Since $\overline{h_{1}}=\overline{\zeta_{1}}=0$ at $x=0$ and $\overline{h_{1 x}}=\overline{\zeta_{1_{x}}}=\bar{v}$ this reduces to the $0(\epsilon)$ expression

$$
\left(\zeta_{1}^{-}-\zeta_{T}^{-}\right)=-\bar{v} \epsilon
$$

Combining the above two expressions yields

$$
\zeta_{1}^{-}-\left(\zeta_{1}^{-}-\zeta_{T}^{-}\right)=\zeta_{1}^{+}
$$

or

$$
\zeta_{T}^{-}=\zeta_{1}^{+} \quad \text { at } \quad x=0 .
$$

On the one-layer side of the surface front, $\zeta_{1}^{+}$is just equal to $\zeta_{T}^{+}$, so this boundary condition becomes

$$
\zeta_{T}^{-}=\zeta_{T}^{+} \quad \text { at } \quad x=0 .
$$

Continuity of normal velocity can be expanded in the same manner to give at $0(\epsilon)$

$$
u_{2}^{-}=u_{2}^{+} \quad \text { at } \quad x=0
$$

or using (5.6a)

$$
\sigma \zeta_{T_{z}}^{-}-l \zeta_{T}^{-}=\sigma \zeta_{T_{z}}^{+}-l \zeta_{T}^{+}
$$

Combining this expression with (5.11) yields the final boundary condition for $\zeta_{T}$,

$$
\zeta_{\boldsymbol{T}_{\mathbf{z}}}^{-}=\zeta_{T_{\boldsymbol{x}}}^{+} \quad \text { at } \quad x=0 .
$$

The right-hand-side can be obtained by differentiating (5.9). This matching condition can now be incorporated into the numerical scheme by approximating $\zeta_{T_{x}}$ by a finite difference.

The above discussion was for an isolated front. This type of model is relevant to many oceanic fronts and was the subject of the work of Killworth et al. (1984). The emphasis of this study is the coastal upwelling front which requires a coastal barrier for its existence. Further, coastal upwelling fronts often occur over bottom topographies which are not flat (Fig. 5.1b). 
These features require a different treatment of the boundary conditions in the single-layer region $\left(x>x_{f}\right)$. The origin of the coordinate system has returned to the coastal wall as defined in Fig. 5.1b. Note that the boundary conditions as $x \rightarrow-\infty$ remain the same for either model. The boundary condition at $x=0$ is no flow through the coastal barrier

$$
u_{2}^{+}=0
$$

or using (5.6a)

$$
\sigma \zeta_{T_{x}}^{+}=l \zeta_{T}^{+} .
$$

For a flat bottom the solution to (5.7b) which satisfies (5.13) can again be found analytically and matched onto the two-layer region as outlined above. Another approach is to extend the numerical domain through the front all the way to the coastal barrier and to apply (5.13) at $x=0$ using standard finite difference techniques. This method automatically satisfies continuity of properties across $x=x_{f}$ and has the advantage of allowing solutions for bottom topographies which are not flat. This latter approach is used in the numerical solutions presented here.

The final boundary condition required to specify the problem uniquely is applied to the upper layer equation at the front. It was noted previously that the governing equation (5.7a) was singular at $x=x_{f}$ so one way to express the boundary condition is that $\zeta_{1}$ remain finite at the front. This qualitative condition must be made quantitative in order to implement it in a numerical scheme. This is done by realizing that the surface front must be a material surface. This can be expressed mathematically as

$$
\frac{d}{d t}\left[x-\left(x_{f}+\epsilon\right)\right]=0
$$

or

$$
\left(\frac{\partial}{\partial t}+u_{1} \frac{\partial}{\partial x}+\bar{v} \frac{\partial}{\partial y}+v_{1} \frac{\partial}{\partial y}\right)\left[x-\left(x_{f}+\epsilon\right)\right]=0
$$


where again $\epsilon$ is the small displacement of the surface front from its equilibrium position at $x=x_{f}$. By definition

$$
u_{1}=\frac{d x}{d t}
$$

so $(5.14)$ becomes

$$
u_{1}-\left(\frac{\partial}{\partial t}+u_{1} \frac{\partial}{\partial x}+\bar{v} \frac{\partial}{\partial y}+v_{1} \frac{\partial}{\partial y}\right)\left(x_{f}+\epsilon\right)=0 .
$$

This can be expanded in a Taylor series about the unperturbed frontal location and linearized to $0(\epsilon)$ to obtain

$$
u_{1}-\left(\frac{\partial}{\partial t}+\bar{v} \frac{\partial}{\partial y}\right) \epsilon=0 \quad \text { at } \quad x=x_{f} .
$$

Assuming the perturbation frontal displacement $(\epsilon)$ has normal mode form as in (5.1) this becomes

$$
u_{1}+i(\sigma-l \bar{v}) \epsilon=0 \quad \text { at } \quad x=x_{f} .
$$

Again using the $O(\epsilon)$ version of vanishing layer depth (5.10) and dropping the minus superscript this can be written as

$$
u_{1}+\frac{i(\sigma-l \bar{v})\left(\zeta_{T}-\zeta_{1}\right)}{\bar{v}}=0 \quad \text { at } \quad x=x_{f} .
$$

Substituting $u_{1}$ from (5.5a) this boundary condition becomes

$$
\begin{gathered}
\bar{v} \zeta_{1_{x}}-\left(1+\bar{v}_{x}\right) \zeta_{1}+\left(1+\bar{v}_{x}\right) \zeta_{T}= \\
\text { at } \quad x=x_{f} .
\end{gathered}
$$

This boundary condition derived from assuming that the surface front is a material surface is exactly the same as the governing equation (5.7a) expressed at $x=x_{f}$ where $\overline{h_{1}}=0$. That this derivation based on physical reasoning reduces to the governing equation suggests that, even though 
this is a singular point, the upper layer equation should remain regular and $\zeta_{1}$ should be well behaved at the front. The redundancy between the boundary condition and the governing equation leads to difficulties in an analytic attempt to solve (5.7a). Previous studies [e.g. Killworth (1983); Killworth et al. (1984)] avoid this difficulty by using an integral solution technique which accommodates the regularity at the front rather than explicitly applying a boundary condition there. In the numerical solution technique used here the expression (5.15) is simply finite differenced to the same accuracy as the rest of the numerical domain and evaluated at the front. Results indicate that this works well.

One final consideration is the special case of a uniform potential vorticity basic state flow. From (4.5b) uniform potential vorticity requires $1+\bar{v}_{x}=0$ at the front so $(5.15)$ becomes

$$
(\sigma-l \bar{v}) \zeta_{1_{x}}=l \zeta_{1} \quad \text { at } \quad x=x_{f}
$$

This boundary condition is different from the upper layer equation expressed at $x=x_{f}$. This can be demonstrated by writing $(5.7 \mathrm{a})$ in terms of $Q_{1}$ as defined by $(4.5 \mathrm{~b})$

$$
\begin{aligned}
& \zeta_{1 x z}-\frac{Q_{1_{x}}}{Q_{1}} \zeta_{1_{x}}-\left(1+\overline{h_{1}} l^{2}\right) Q_{1} \zeta_{1}+Q_{1} \zeta_{T}= \\
& \frac{l \bar{v}}{\sigma}\left\{\zeta_{1_{x x}}-\frac{Q_{1_{x}}}{Q_{1}} \zeta_{1_{x}}-\left[\frac{Q_{1_{x}}}{Q_{1} \bar{v}}+\left(1+\overline{h_{1}} l^{2}\right) Q_{1}\right] \zeta_{1}+Q_{1} \zeta_{T}\right\}
\end{aligned}
$$

and then setting $Q_{1}=1$ and $Q_{1_{x}}=0$ to obtain

$$
(\sigma-l \bar{v})\left[\zeta_{1_{x x}}-\left(1+\overline{h_{1}} l^{2}\right) \zeta_{1}+\zeta_{T}\right]=0 .
$$

If $\sigma \neq l \bar{v}$ this can be expressed at $x=x_{f}$ where $\overline{h_{1}}=0$,

$$
\zeta_{1_{x z}}-\zeta_{1}+\zeta_{T}=0
$$

This expression is clearly different from (5.16). In fact, (5.16) is just the result of requiring the coefficient of $Q_{1_{x}} / Q_{1}$ to vanish. In other words, 
a front with uniform basic state potential vorticity allows the requirement that the front be a material surface to provide a unique boundary condition which can be incorporated into the numerical scheme by standard finite difference techniques.

The actual solution of (5.7) with the appropriate boundary conditions as detailed above proceeds via (5.8) by first choosing a basic state flow as given by (4.28). Choices are then made for the offshore distance to the surface front $\left(x_{f}\right)$, bottom topography and/or lower layer depth far offshore $\left(\mathrm{H}_{2}\right.$; see Fig. 3.1). These choices completely specify the basic state and model geometry. This allows (5.8) to be solved for the complex eigenvalue $(\sigma)$ and the complex eigenfunctions $(\underset{\sim}{\eta})$ for a given alongfront wavenumber $(l)$. The value of $l$ is varied to investigate the potential instability of the basic state to perturbations of a given alongfront scale. The analyses can then be repeated for different choices of the basic state flow or model geometry.

\subsection{Inviscid, Flat-Bottom Model}

In this section the solution technique just described is applied to a simple model geometry consisting of a flat bottom adjacent to a coastal wall (Fig. 5.2). A surface front lying over a flat bottom is relevant to many geophysical phenomena, but it must be remembered that the distinguishing feature of many coastal upwelling fronts is the presence of strongly sloping bottom topography. This more realistic geometry will be addressed in the next section. Here, the stability properties of various basic state flows over a flat bottom will be discussed in detail. Initially a uniform potential vorticity basic state flow will be considered followed by a discussion of the effect of a finite mean potential vorticity gradient.

The choice of the basic state interface profile is motivated by several considerations. The intent is to select a model state which represents a fully mature coastal upwelling front. The density and flow structure arises 


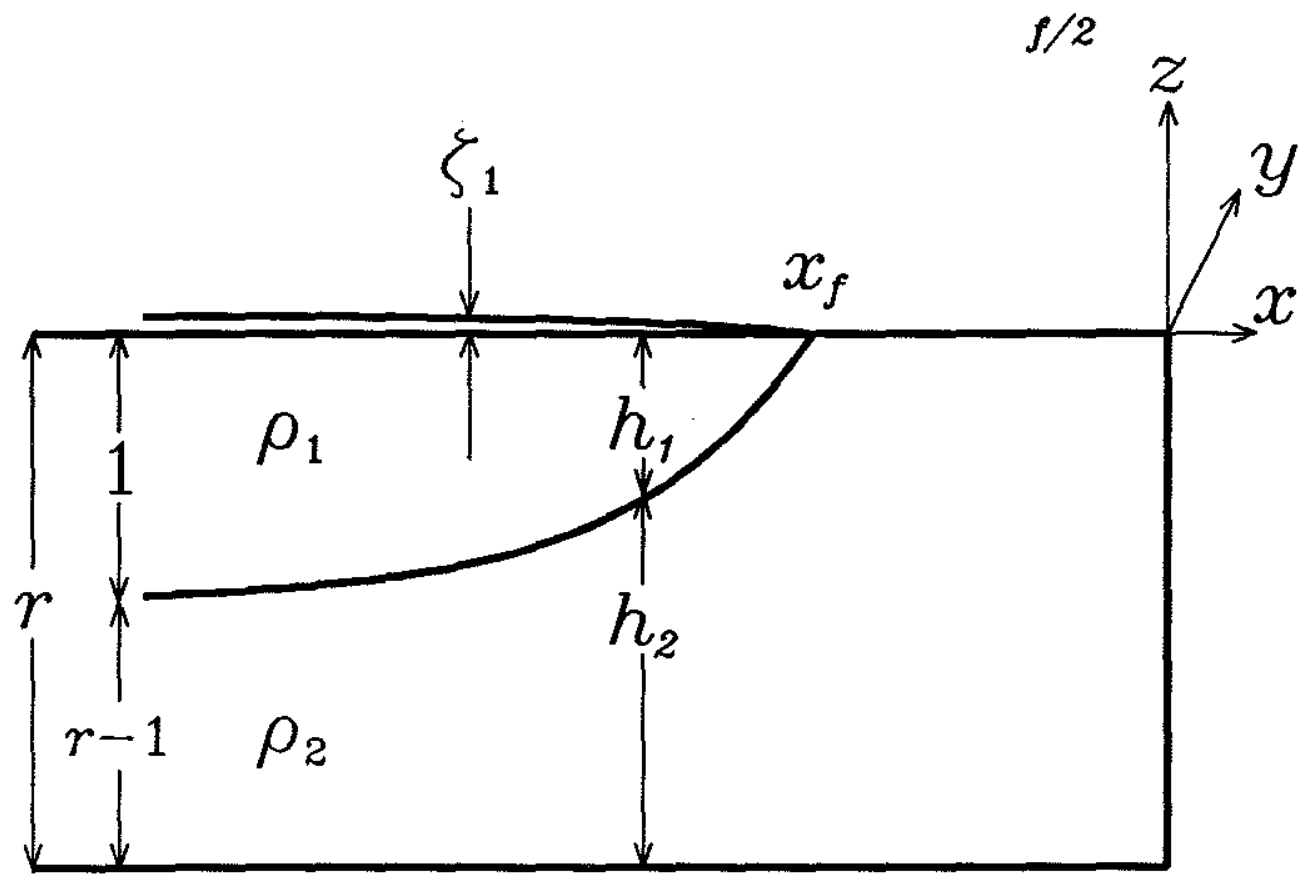

Figure 5.2: Flat-bottom frontal model geometry. The constant offshore depths of the layers are normalized by the depth of the upper layer $\left(H_{1}\right.$ in Fig. 3.1) so that the upper layer depth is equal to one and the total depth becomes $r=\left(H_{1}+H_{2}\right) / H_{1}$. 
from the upwelling process described in chapter 2. Essential to this formation process is an alongshore wind stress. The model developed here explicitly leaves out wind stress and considers the stability of a basic state which is the end product of an alongshore wind event. Another point is the lack of cross-frontal mixing which is likely an important physical process in the formation and evolution of a coastal upwelling front (e.g. Garvine, 1979a, b, 1980). One obvious way to choose a basic state configuration is to use observations to specify an interface profile. This may be the most realistic, but can be problematic for several reasons. Subsurface observations of density, particularly vertical sections perpendicular to the front, are often not available for the coastal upwelling front of interest. Even without detailed knowledge of the subsurface density structure, it would still be useful to predict the scales of this variability based on an instability model whose inputs (e.g. layer depths, density difference between layers) can be set using historical data or a few hydrographic casts. When observations are available, they include small scale features in density due to mixing, internal waves and mixed layer dynamics. These complicate the specification of a basic state and may obscure the understanding of the stability of the front to larger scale processes. Finally, observations of subsurface properties in frontal regions are never truly synoptic which may create problems in specifying a correct basic state configuration.

Another approach is to consider interface profiles which arise from simple models of the coastal upwelling process. Many such models exist as briefly reviewed in chapter 2. A particularly simple model is that of Csanady (1977) for a flat-bottom two-layer ocean. The upwelling of the interface due to an alongshore wind stress is developed by considering the conservation of potential vorticity in each layer. The resulting interface profile is exponential with an e-folding scale equal to the internal Rossby radius of deformation. The upwelled state has uniform potential vorticity in the two layers as a necessary result of conserving potential vorticity 
throughout the upwelling process. This model oversimplifies the formation process, but certainly provides a realistic physical explanation for the shape of a coastal upwelling front. A great number of observational studies have suggested that the internal Rossby radius is a relevant decay scale to consider. An effort to select a simple, yet physically realistic basic state configuration based on this type of upwelling model led to the selection of the exponential profiles given by (4.28). Interface profiles evident in vertical sections of density for many coastal upwelling fronts appear to be realistically represented by the exponential form employed here. In particular, a uniform potential vorticity basic state flow in the upper layer will be considered first. Since no mean flow exists in the lower layer, the potential vorticity there will be dictated by the change in layer depth. The presence of unstable waves in a system with this distribution of potential vorticity (i.e. lack of a change in sign of the potential vorticity gradient) will distinguish these ageostrophic flows from traditional quasi-geostrophic dynamics.

The stability of this uniform basic state potential vorticity model with a flat bottom as sketched in Fig. 5.2 will now be presented. The depth of the upper layer far from the surface front $\left(H_{1}\right.$ in Fig. 3.1) will be chosen to be always equal to one. Using this, the total depth $H=H_{1}+H_{2}$ can be denoted by $r$ so that the lower layer depth is equal to $r-1$ (Fig. 5.2). The stability theorem (4.27) suggests that this basic state will be unstable if the lower layer is sufficiently shallow. The first model geometry considered will have equal upper and lower layer depths, $r=2$, and the surface front will be located two Rossby radii from the coastal barrier $\left(x_{f}=-2 \mathrm{R}\right)$. This model geometry with uniform potential vorticity flow will serve as a standard case with which to compare the results for models with different parameter choices.

Given the basic state flow and the model geometry, the governing equations (5.7) are solved for a range of alongfront wavenumbers $(l)$. The com- 
plex frequency $(\sigma)$, which is the eigenvalue for the problem, yields two important properties of the unstable wave. The imaginary part of $\sigma$ is the rate at which the unstable wave will grow with time (see 5.2). The growth rate $\left(\sigma_{i}\right)$ is plotted as a function of alongfront wavenumber in Fig. 5.3a. No unstable modes exist above $l=l_{c} \simeq 1.50$ where $\sigma_{i}=0$. This "short wave cutoff" is related to restricting the vertical shear in the system to occur across an infinitely thin layer at the interface and is a common feature of stability models which use homogeneous fluid layers (Pedlosky, 1986). The wave with the largest growth rate occurs at $l=l_{m}=1.05$ where $\sigma_{i_{m}}=0.09$ (the subscript $m$ identifies the maximum growth rate). Within the limits of the linear, small amplitude theory presented here, this is the wave most likely to be observed as its amplitude grows most rapidly from an initial condition containing many wave components. As the disturbance grows to finite amplitude, nonlinear effects may alter the properties of the fastest growing wave.

The real part of the eigenvalue $\left(\sigma_{r}\right)$ divided by the wavenumber $(l)$ yields the phase speed of the unstable wave $\left(c_{r}\right)$. This quantity is plotted as a function of alongfront wavenumber in Fig. 5.3b. The solid curve for $l<l_{c}$ is for the unstable mode whose growth rate is given in Fig. 5.3a. The values for $c_{r}$ are all negative which correspond to propagation to $-y$. This downstream direction for phase propagation can be anticipated by invoking a common guideline for the phase speed of unstable waves. In general, the phase speed of an unstable wave must lie within the range of the mean flow speeds. These so-called "semi-circle" theorems have been rigorously derived for many layer models of instability both using quasigeostrophic dynamics (Pedlosky, 1964) and ageostrophic dynamics (HY). Since $\bar{v}$ is in the $-y$ direction the unstable wave should propagate in the same direction with a phase speed less than the maximum flow speed. This result is confirmed in Fig. 5.3b, remembering that maximum flow speed occurs at the front $\left(x=x_{f}\right)$ and is equal to $-\alpha$, which for the uniform 

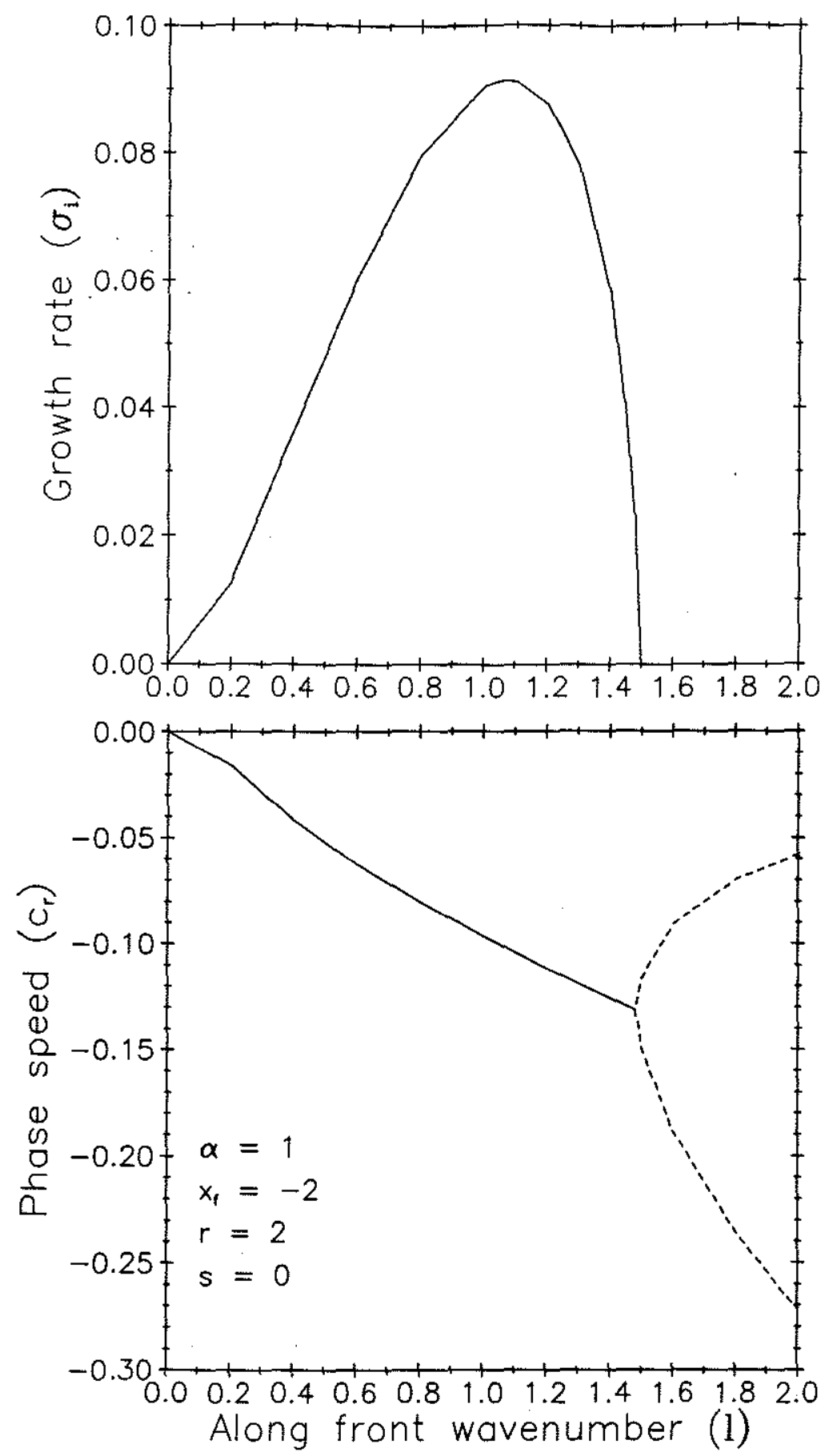

b

Figure 5.3: (a) Growth rate versus alongfront wavenumber for the unstable mode present on a model front with uniform basic state upper layer potential vorticity. (b) Real part of the phase speed versus alongfront wavenumber for the unstable mode $(l<1.5$, solid curve) and for two stable modes $(l \geq 1.5$, dashed curves). 
potential vorticity case considered here is equal to -1 . At $l=l_{c}$ the real part of the phase speed splits into two stable waves (dashed curves) which for $l<l_{c}$ had been resonating to produce the unstable mode. In addition to these stable waves, there exist many more stable vorticity modes with $c_{r}<0$ which are not plotted in Fig. 5.3b.

Before expanding on this idea of resonating modes and taking a look at the structure of the unstable wave, it is useful to consider the dimensional properties of the fastest growing wave. The length, time and velocity scales used for the redimensionalization are defined in the beginning of chapter 3 . To find the wavelength of this mode multiply $l_{m}^{-1}$ by $2 \pi R$. The e-folding period [the time it takes the perturbation to grow to $\exp (+1)$ of its initial amplitude] is obtained by multiplying $\sigma_{i_{m}}^{-1}$ by $f^{-1}$. Finally, the dimensional phase speed is recovered by multiplying $c_{r}$ by $R f$. For $R=5 \mathrm{~km}$ and mid-latitudes the fastest growing wave has a wavelength of $\sim 30 \mathrm{~km}$, an e-folding period of $\sim 1.5$ days and a phase speed toward $-y$ of $\sim 3 \mathrm{~cm} \mathrm{~s}^{-1}$. This standard model, which has not been tuned to the configuration of a coastal upwelling front for any particular geographic location, yields spatial scales for the fastest growing wave which are of the same order as the observed scales of alongfront variability off, for instance, the coast of Oregon as displayed in Fig. 1.2 (O'Brien et al., 1974). Values for growth rates and alongfront phase speeds are more difficult to obtain from observations, but when estimates of these properties are available (e.g. Petrie et al., 1987) they are comparable to the values predicted by this simple instability model. To make a more detailed comparison between the model-derived properties of the fastest growing wave and observations, it is necessary to understand the effect of varying the basic state flow and model geometry. For example, the influence of sloping bottom topography beneath the surface front, a physical characteristic of upwelling fronts in nature, will be investigated. After a discussion of these effects in the remainder of this chapter, a comparison between the model predictions and observed alongfront variability 
will be made in chapter 6 .

The structure of the most unstable wave is displayed in Fig. 5.4. Properties of the upper layer are presented in Fig. 5.4a where the dashed line at $x=-2$ represents the surface front. The solid and dashed curves represent contours of $\zeta_{1}$, the sea surface elevation, whose maximum value occurs at the front and is normalized to 1.0. This normalization is done because the linear stability analyses presented here does not allow the amplitude of the unstable disturbance to be determined. The perturbation upper layer velocity field follows from (5.5). For presentation purposes, the velocities are scaled such that the vector beneath the legend represents one velocity unit. Lower layer velocities and contours of $\zeta_{T}$ [given by (3.10) and is essentially the lower layer "pressure"] appear in Fig. 5.4b. The normalization and scaling procedure is identical for the two layers so that comparisons of properties between them can be made.

All the fields are frontally-trapped with a cross-front e-folding scale of $\sim 1$ Rossby radius $(R)$. Upper layer velocities exhibit a pattern consistent with a wavelike deflection of the surface front. If the velocity field were completely geostrophic the contours of $\zeta_{1}$ would serve as streamlines for the flow. The crossing of $\zeta_{1}$ contours by the velocity vectors, especially near the surface front, is indicative of a significant ageostrophic component to the velocity field. Velocities in the lower layer are much weaker and more geostrophic than those in the upper layer. The velocity and $\zeta_{T}$ fields form closed cells with maxima occurring $\sim 0.75 R$ to the two-layer side of the surface front.

As is apparent from the discussion in chapter 4, the interpretation of energy transfers within an unstable system can be thought of in two ways. In traditional instability theory the flow of energy to the unstable disturbance from the basic state and vice versa can be analyzed. For nondivergent flow fields instability requires that the sum of the energy conversion must result in the flow of energy from the basic state to the disturbance [positive 

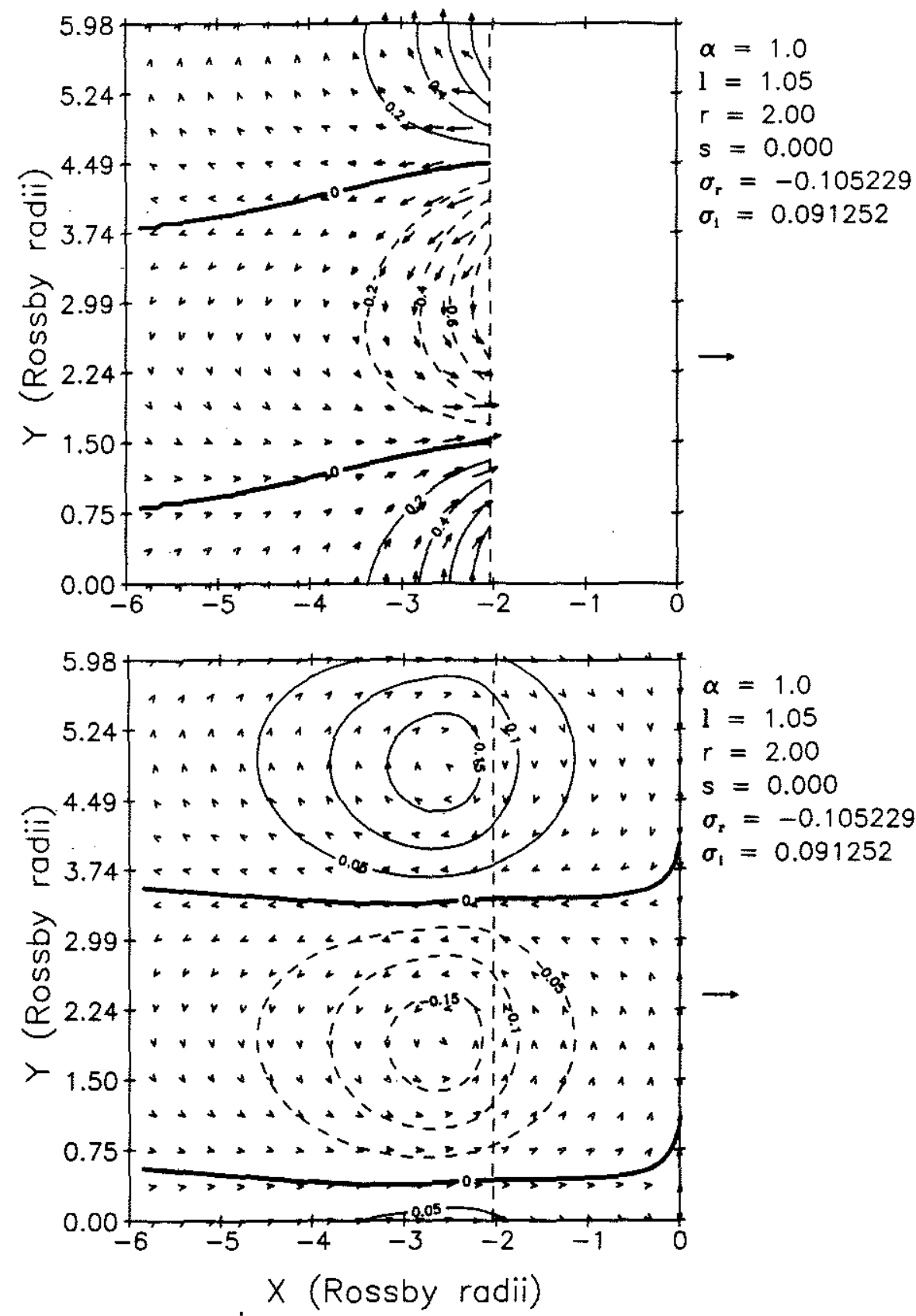

Figure 5.4: (a) Perturbation upper layer velocities and contours of sea surface elevation $\left(\zeta_{1}\right)$ for one wavelength of the fastest growing wave present on a model front with uniform basic state upper layer potential vorticity. The upper layer only exists offshore of the surface front represented by a dashed line along $x=-2.0$. The maximum value of the sea surface elevation occurs at the surface front and is normalized to 1.0. Velocities are scaled such that the vector beneath the legend represents one velocity unit. (b) Perturbation lower layer velocities and contours of lower layer "pressure" $\left(\zeta_{T}\right)$. 
wave energy $\left(E_{2}^{\star}\right)$ and negative mean energy $\left.\left(E_{1}^{\star}\right)\right]$. For the flows considered here it was shown (see chapter 4 ) that unstable waves can exist even if the net flow of energy between the basic state and the disturbance is zero or even when the transfer is from the disturbance to the basic state flow creating negative wave energy and positive mean energy. These cases are certainly counterintuitive, but as described in chapter 4 it is not difficult to find physical systems which can be unstable without the flow of energy from the basic state to the disturbance (e.g. an inverted pendulum). The instabilities found here on a uniform basic state potential vorticity flow will have zero net energy flow between the basic state and disturbance fields (see 4.20). Nevertheless, it still is useful to analyze the energy conversion terms as described in conjunction with (4.13) to understand the primary physical mechanism for the instability (e.g. baroclinic or barotropic conversion).

The other interpretation of instability involves the exchange of disturbance energies between two resonating stable modes. The unstable mode will grow with time keeping its disturbance (mean plus wave) energy equal to zero. The flow of energy in this case is between the two stable partners. Depending on the basic state configuration, the unstable mode may gain from, lose to or leave unchanged (as is the case here for a uniform potential vorticity flow) the energy of the basic state flow. A good description of these ideas can be found in HY. Since both interpretations yield useful information, both will be considered here.

The contours of $\zeta_{1}$ and $\zeta_{T}$ provide phase information for the unstable mode. The sense of phase tilt in an unstable disturbance is useful in interpreting the direction and mechanism of energy transfer in the system (Pedlosky, 1986). In chapter 4 the possibility of conversion of energy via Reynolds stresses and baroclinic instability was mentioned. The signature of an unstable wave extracting energy from the basic state potential energy field via baroclinic instability is that the wave in the upper layer lags the wave below. This sense of phase tilt, such that the perturbation is "leaning" 
against the basic state vertical shear, is apparent in Fig. 5.4 and it may be anticipated that the term on the right-hand-side of (4.13) representing this process will be positive. The sign of the conversion of kinetic energy via the horizontal Reynolds stress is more difficult to determine by just inspecting Fig. 5.4a. The first horizontal Reynolds stress term on the right-hand-side of (4.13) will be positive (conversion of basic state kinetic energy to the perturbation) if the phase of the perturbation is again leaning against the basic state horizontal shear. This is clearly the case in Fig. $5.4 \mathrm{a}$, but the presence of the additional term in (4.13) proportional to the horizontal Reynolds stress, but with opposite sign, complicates the interpretation. In fact, for this uniform basic state potential vorticity case the wave energy should be zero from (4.20), so if the baroclinic conversion is from the basic state flow to the perturbation then the conversion of kinetic energy via the Reynolds stress must be of the opposite sign. A numerical evaluation of the energy balance presented below does indeed confirm this result.

The solutions obtained numerically can be used to calculate explicitly the terms in the energy statements of chapter 4 . The following calculations were performed using the full energy $E_{2}^{\star}$ including the geostrophic and ageostrophic parts. The terms in the definition of wave energy (4.9d) are plotted as a function of cross-front distance in Fig. 5.5. The solid curve is the positive definite sum of the kinetic energy in each layer and the potential energy. The long dashed curve is the correlation between $v_{1}$ and interface displacement which results in a negative contribution to the wave energy. This results because the unstable disturbance decreases the total speed $\left(v_{1}>0\right)$ where it deepens the upper layer $\left[\left(\zeta_{1}-\zeta_{T}\right)>0\right]$. The sum of these positive and negative contributions to the wave energy is plotted as the short dashed curve in Fig. 5.5. Its integral over the domain of the fluid vanishes, confirming the result that unstable waves on basic state flows with uniform potential vorticity have "zero wave energy".

The energy conversion terms in (4.13) due to the action of Reynolds 

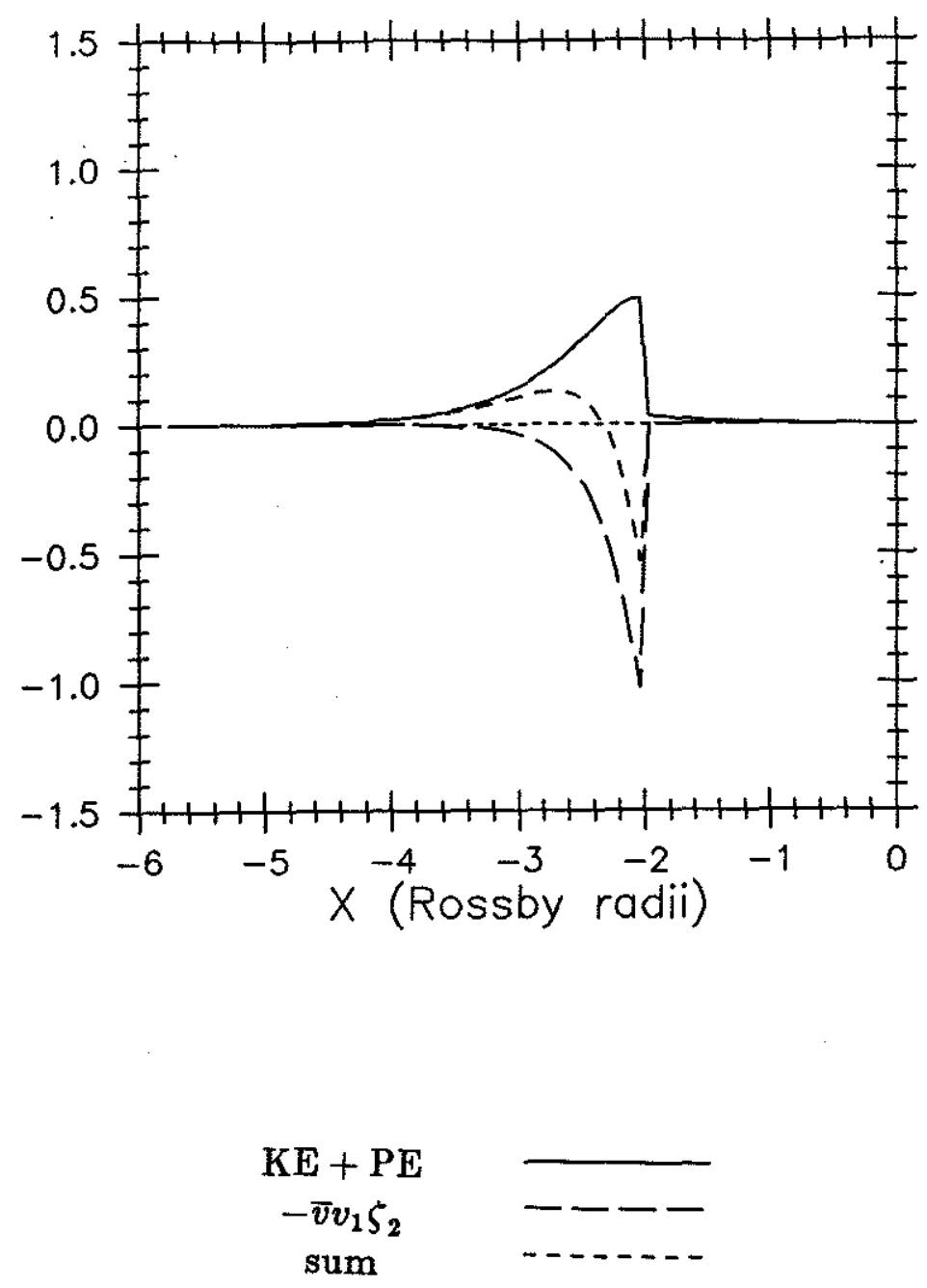

Figure 5.5: Terms in the definition of wave energy (4.9d) versus cross-front distance: kinetic plus potential energy $\overline{h_{1}}\left(u_{1}^{2}+v_{1}^{2}\right) / 2+\overline{h_{2}}\left(u_{2}^{2}+v_{2}^{2}\right) / 2+\zeta_{2}^{2} / 2$ (solid curve); cross-term $-\bar{v} v_{1} \zeta_{2}$ (long-dashed curve) and ; total wave energy $E_{2}^{*}$ (short-dashed curve). 
stresses and baroclinic instability are plotted as a function of $x$ in Fig. 5.6.

The values to the right of the figure result from integrating the various terms over the domain of the fluid. Only the Reynolds stress terms are plotted in Fig. 5.6a. The first term in the integrand of (4.13) representing the horizontal Reynolds stress $-\bar{h}_{1} \bar{v}_{x} u_{1} v_{1}$ is positive as was inferred from the examination of the phase tilt in the unstable disturbance. The second term $-\bar{v}^{2} u_{1} v_{1}$ is negative and large with the result that the net horizontal Reynolds stress acts to convert energy from the perturbation to the mean flow. The vertical Reynolds stress term $-\overline{h_{1}} \bar{v}\left(u_{1_{x}}+v_{1_{y}}\right) v_{1}$ is positive and the final term in the integrand of (4.13), which earlier had been related to the deflection of the surface front, is large and negative. The sum of the Reynolds stress terms plotted in Fig. 5.6a is plotted as the dashed curve in Fig. 5.6b. The solid curve in Fig. $5.6 \mathrm{~b}$ represents the baroclinic energy conversion term, whose integral over the domain of the fluid is positive and exactly balances the integral of the sum of the other terms. The integrand in (4.13) can be related to the mean energy $E_{1}^{\star}$ through the use of (4.10). Specifically, $\partial E_{1}^{\star} / \partial t=-\partial E_{2}^{\star} / \partial t=\int($ energy conversion terms $) d A$. The fact that the sum of the terms in the integrand is zero confirms that the mean energy as well as the wave energy (as illustrated in Fig. 5.5) are zero for this basic state flow with uniform potential vorticity. The ratio of the magnitude of the baroclinic conversion term to the magnitude of the Reynolds stress terms [neglecting the contribution from the term, $-\bar{v} \bar{v}_{x} u_{1}\left(\zeta_{1}-\zeta_{T}\right)$, which is related to the deflection of the surface front] is $\sim 2.6$ which indicates that the energy conversion process, although mixed, is primarily baroclinic.

The results for momentum confirm the signs inferred from (4.23). Even though $Q_{1_{z}}=0$, the gradient of the lower layer basic state potential vorticity $\left(Q_{2}\right)$ is nonzero and negative. The calculated value for the wave momentum $\left(M_{2}^{\star}\right)$ from $(4.12 \mathrm{~d})$ is positive while that of the mean momentum $\left(M_{1}^{\star}\right)$, from (4.22) and the fact that $\partial M_{1}^{\star} / \partial t=-\partial M_{2}^{\star} / \partial t$, is negative. 


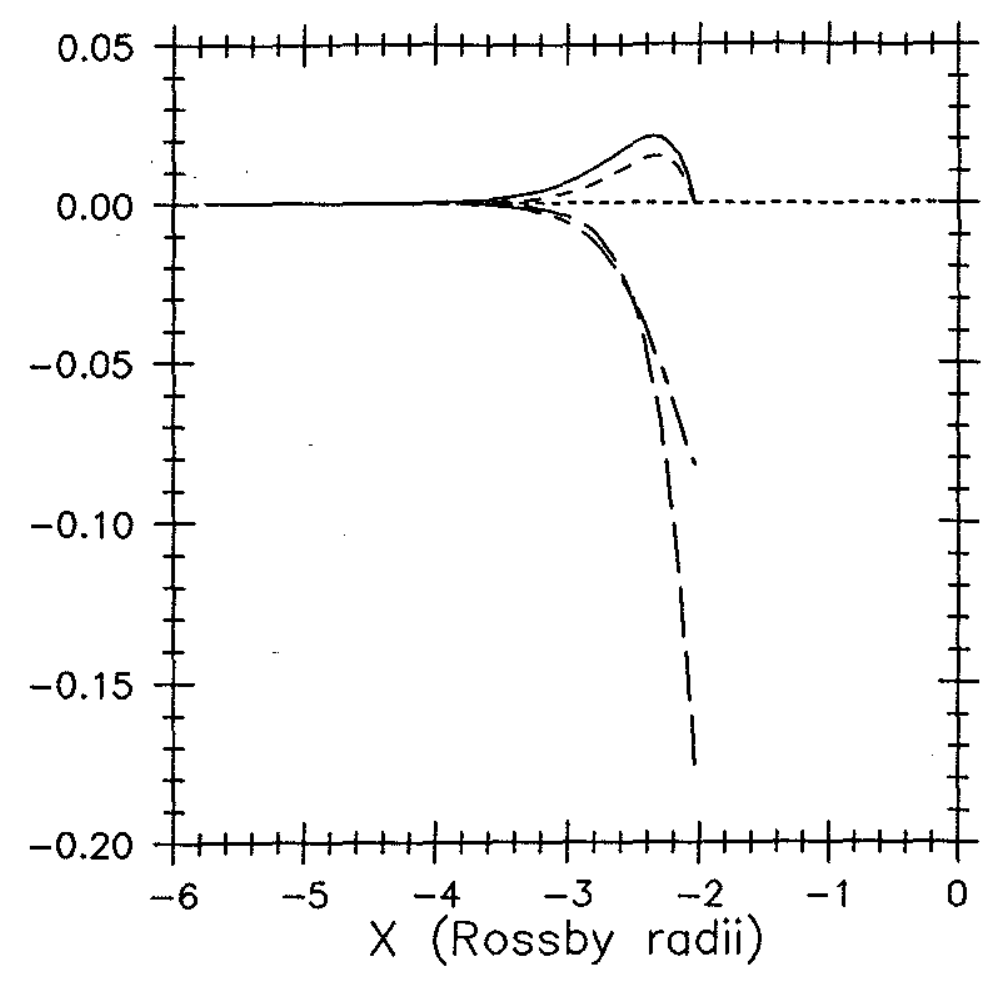

a

$$
\begin{array}{ccr}
-\bar{h}_{1} \bar{v}_{x} u_{1} v_{1} & =0.0162 \\
-\bar{v}^{2} u_{1} v_{1} & = & -0.0479 \\
-\overline{h_{1}} \bar{v}\left(u_{1_{x}}+v_{1_{y}}\right) v_{1} & = & 0.0098 \\
\overline{v v}_{x} u_{1} \zeta_{2} & = & -0.0354
\end{array}
$$

Figure 5.6: Energy conversion terms defined in (4.13) versus cross-front distance: (a) horizontal Reynolds stresses $-\bar{h}_{1} \bar{v}_{x} u_{1} v_{1}$ (solid curve) and $-\bar{v}^{2} u_{1} v_{1}$ (long-dashed curve); vertical Reynolds stress $-\overline{h_{1}} \bar{v}\left(u_{1_{x}}+v_{1_{y}}\right) v_{1}$ (short-dashed curve); $\bar{v} v_{x} u_{1} \zeta_{2}$ (dot-dashed curve); (b) conversion of energy via baroclinic instability $-\bar{v} u_{1} \zeta_{2}$ (solid curve) and ; the sum of the terms plotted in (a) (dashed curve). 


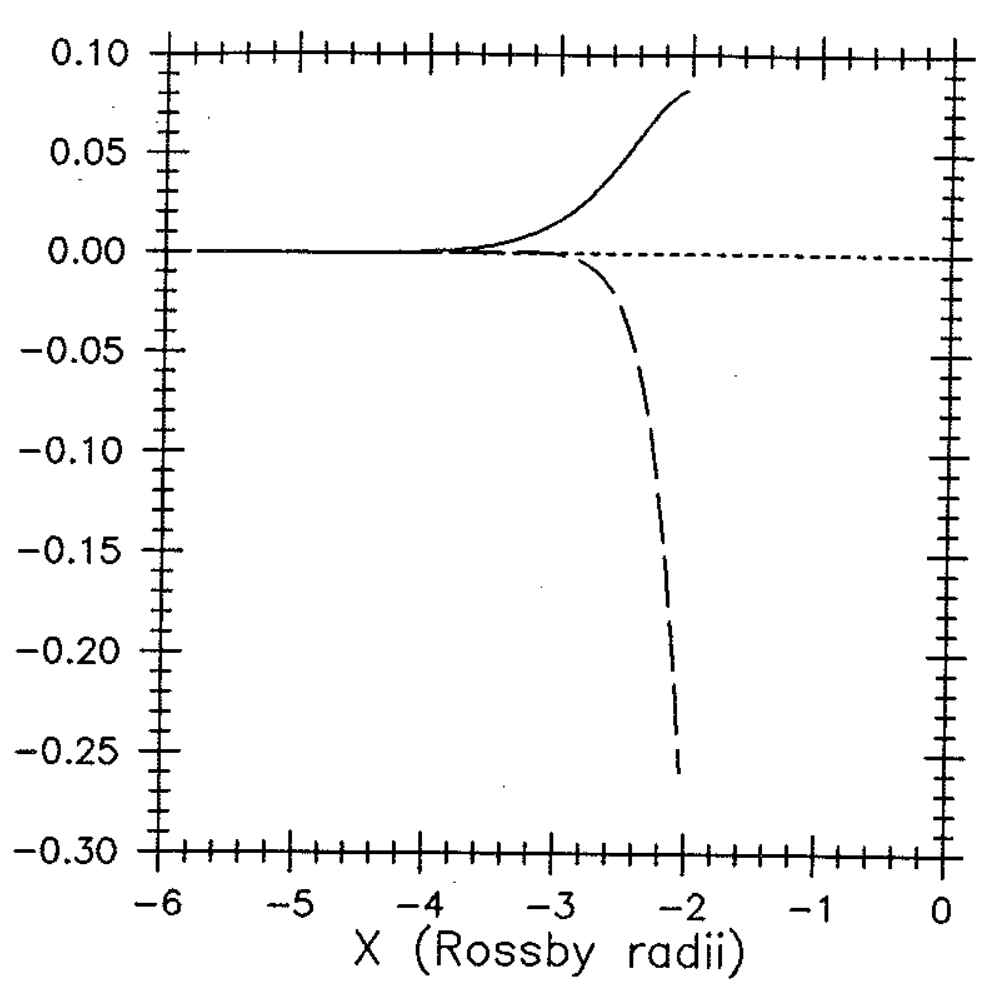

b

$$
\begin{array}{rrr}
-\bar{v} u_{1} \zeta_{2} & =0.0573 \\
\text { sum } & = & -0.0573
\end{array}
$$


These two exactly balance such that the total disturbance (mean plus wave) momentum is zero.

The structures of the two stable waves at $l=2.0$, which for $l<l_{c}$ had been resonating to produce the unstable mode, are presented in Fig. 5.7. The velocities are again scaled for each wave such that the vector beneath the legend represents one unit. Both stable waves are frontally-trapped and have highly ageostrophic velocity fields. The wave with $\sigma_{r}=-0.5444$ (or $c_{r}=-0.2722$ ) (Fig. 5.7a) is primarily trapped in the upper layer while the wave with $\sigma_{r}=-0.1155$ (or $c_{r}=-0.0578$ ) (Fig. 5.7b) has comparable velocity magnitudes in each layer (a more "barotropic" mode). The two waves have the same sign of phase propagation, but oppositely directed group velocities (defined by $c_{g}=\partial \sigma / \partial l$ ). The wave with $\sigma_{r}=-0.5444$ has a negative group velocity and thus propagates energy toward $-y$. The opposite is true for the other stable wave. When the stable waves have the identical wavenumber and phase speed they can resonate to produce the unstable mode. The fact that the stable waves have different energy propagation directions allows for the transfer of energy between them to form a growing unstable disturbance. This idea is elaborated on below.

An explicit calculation of the terms in the energy statements of chapter 4 can also be performed on the two stable modes whose alliance results in the unstable mode analyzed above. The wave with $\sigma_{r}=-0.5444$ has negative disturbance energy while the wave with $\sigma_{r}=-0.1155$ has positive disturbance energy. As described above, when these two waves have the same wavenumber and phase speed they can exchange disturbance energies to form an unstable mode which has zero disturbance energy. Thus, the unstable mode can grow through a transfer of disturbance energy from one of its partners to the other.

The previous paragraphs detail the existence of unstable waves on a uniform basic state potential vorticity flow, confirm the energy statements of chapter 4 and discuss the mechanisms for their existence. The variety 


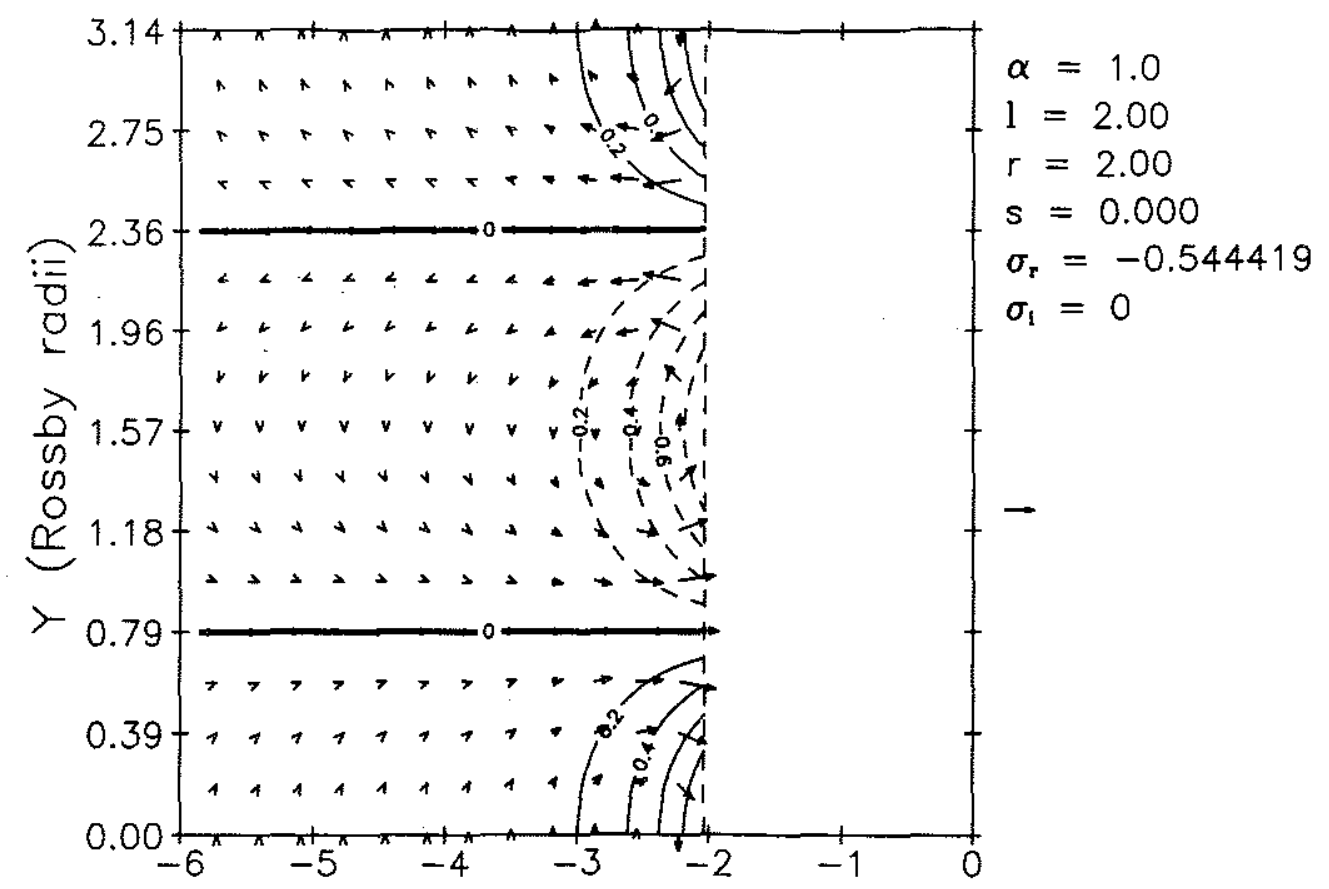

a

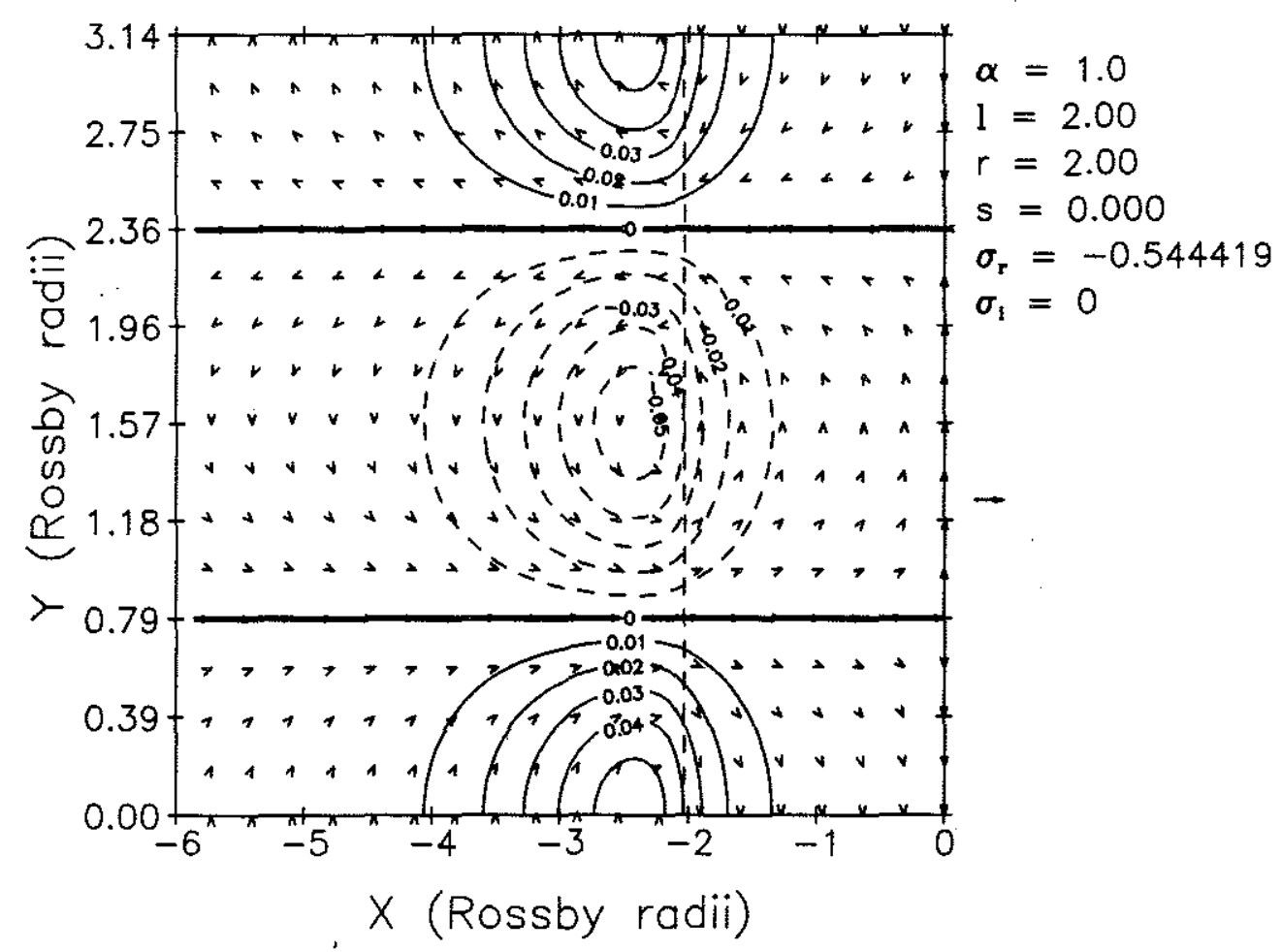

Figure 5.7: Same as in Fig. 5.4 but for two stable waves. (a) Surface-intensified mode and (b) a more "barotropic" mode. 

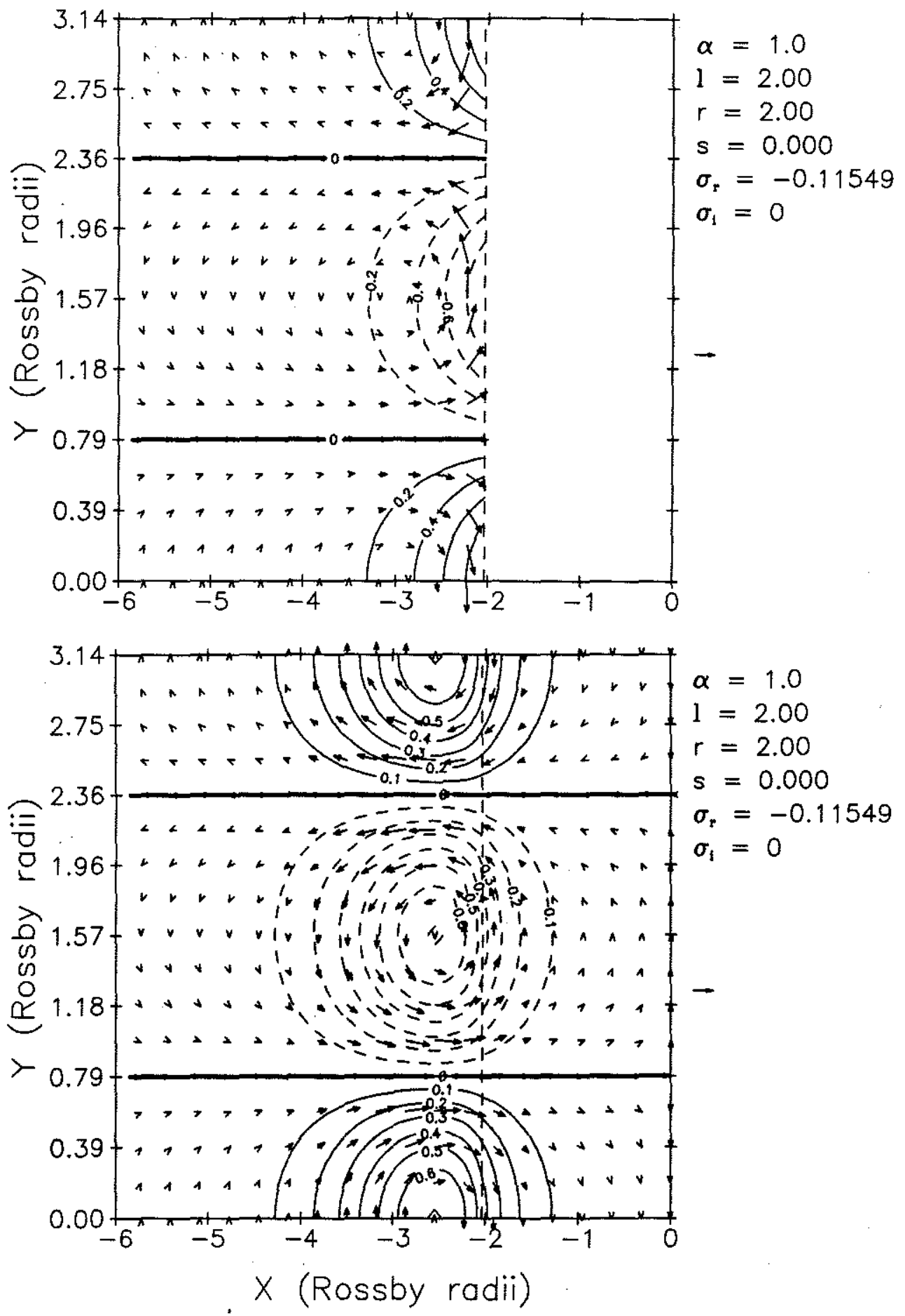
of mean flow profiles and layer depths associated with coastal upwelling fronts found in nature suggests an investigation of the effect of changing the model parameters. The first parameter to be varied is the distance from the front to the coastal wall $\left(x_{f}\right)$. The maximum growth rate $\left(\sigma_{i_{m}}\right)$ is plotted versus $\left|x_{f}\right|$ in units of $R$ in Fig. 5.8. The wavenumber of the fastest growing wave $\left(l_{m}=1.05\right)$ does not change appreciably (less than $1 \%)$ as $\left|x_{f}\right|$ is varied. Moving the front closer to the coastal wall results in a decrease of the maximum growth rate. This decrease is $\sim 34 \%$ for the surface front next to the coastal wall. The growth rate asymptotes to a constant value as $\left|x_{f}\right|$ increases with the choice $\left|x_{f}\right|=2.0 R$ being similar to the no wall case considered by Killworth et al. (1984). In fact, comparison of their numerical results (see their Fig. 4) are in excellent agreement with the results presented in Fig. 5.3a. This agreement also supports the validity of the geostrophic momentum approximation for this choice of basic state flows. In summary, maximum growth rates decrease as the surface front is moved closer than $\sim 2-3 R$ from the coastal wall.

Since the theorem (4.27) establishes the stability of a uniform basic state potential vorticity flow with an infinitely deep lower layer (a reduced gravity model) and the results of this chapter show the existence of a strong instability (large growth rate) for equal layer depths, a dramatic dependence of the growth curve on lower layer depth may be expected. Growth rate versus alongfront wavenumber with $x_{f}=-2.0 R$ for various values of $r$, the nondimensional total depth as defined in Fig. 5.2, is plotted in Fig. 5.9. Deep lower layers decrease the range of unstable wavenumbers, shift the fastest growing wave to longer wavelengths (smaller $l$ ) and dramatically decrease the growth rate of the fastest growing wave. These results are similar to those of Killworth et al. (1984) who, in an investigation concurrent with this study, solved the shallow water equations numerically. Since the source of energy for the unstable wave is primarily baroclinic, the decrease in growth rate can be understood in light of the decreasing over- 


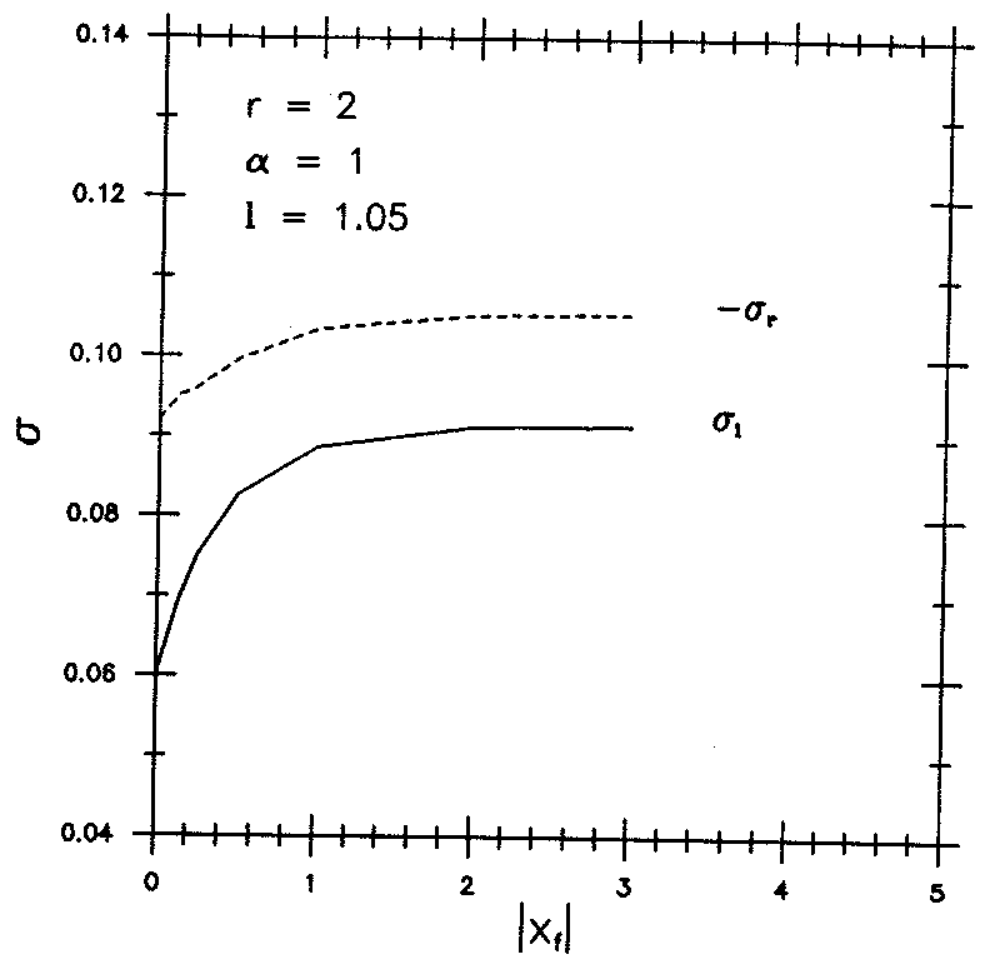

Figure 5.8: Growth rate for the fastest growing wave $\left(l_{m}=1.05\right)$ as a function of $\left|x_{f}\right|$ the distance from the surface front to the coastal barrier. 


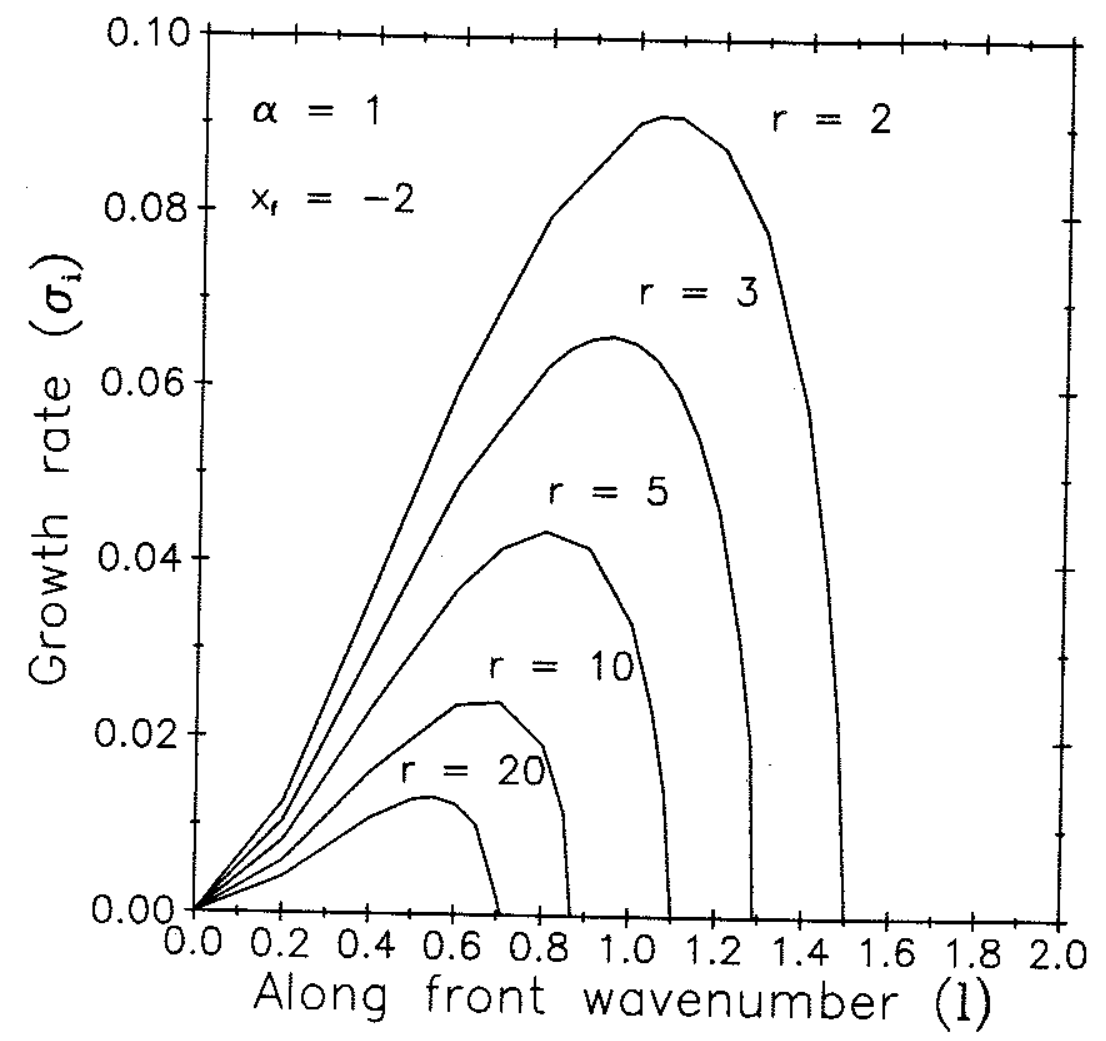

Figure 5.9: Growth rate versus alongfront wavenumber for the unstable modes present on model fronts with varying values of $r$, the scaled total $\operatorname{depth}\left[r=\left(H_{1}+H_{2}\right) / H_{1}\right]$. 
all vertical shear of the basic state flow. For continuous stratification and velocity the growth rate due to baroclinic instability for a given wavenumber is limited by the magnitude of the vertical shear (Pedlosky, 1986). An analogous statement for layer flows may be established (Pedlosky, 1986) using a finite difference form of the vertical shear where $\Delta z$ is the depth of the water column even though, in reality, the vertical shear in the system is concentrated at the interface. As the lower layer depth increases the overall vertical shear decreases and the baroclinic energy conversion process becomes weaker. The absolute stability of the flow with an infinitely deep lower layer occurs essentially because the lower layer becomes so massive that interaction with the upper layer is removed. In other words, the baroclinic conversion of energy is absent and thus the flow becomes stable. The numerical solution presented in Fig. 5.9 is correctly converging to this limit.

The discussion in chapter 4 illustrated the importance of the shape of the interface profile (as denoted by $\alpha$ ) to the stability of a coastal upwelling front. It was also shown that the signs of the wave and mean energy components of the unstable disturbance depend on the signs of the basic state potential vorticity gradients. The effect on the stability properties of the system of varying $\alpha$ will now be addressed. Growth rate versus alongfront wavenumber for three values of $\alpha$ are presented in Fig. 5.10. The wavenumber of the fastest growing wave decreases slightly ( $\sim 5 \%$ for a $20 \%$ increase in $\alpha$ ) as the interface becomes "steeper" (i.e. cross-front efolding scale less than a Rossby radius of deformation). A more significant effect of increasing $\alpha$ is the increase in growth rate of the fastest growing wave. Growth rate $\left(\sigma_{i}\right)$ and the real part of the frequency $\left(\sigma_{r} ;\right.$ not displayed in Fig. 5.10) as a function of $\alpha$ for $l=1.0$ are plotted in Fig. 5.11. The growth rate and the real part of the frequency increase smoothly as $\alpha$ passes through 1.0. Since the growth mechanism is via baroclinic instability, this increase in growth rate is related to the increase in overall vertical shear 


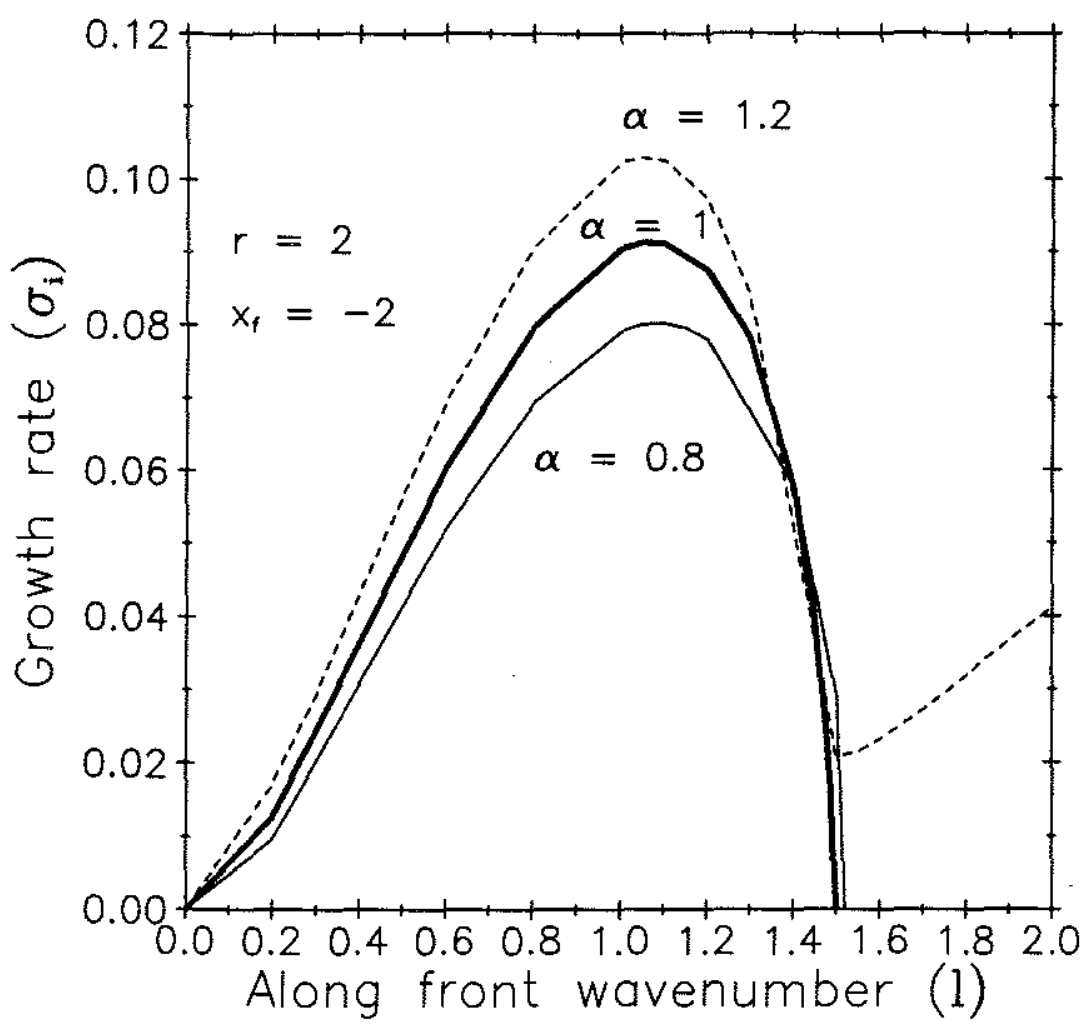

Figure 5.10: Growth rate versus alongfront wavenumber for the unstable modes present on model fronts with three value of $\alpha$, the interface profile parameter. (a) Uniform potential vorticity as in Fig. 5.3a $(\alpha=1.0$, thick solid curve); (b) interface less steeply sloping than in the uniform potential vorticity case ( $\alpha=0.8$, thin solid curve) and (c) interface more steeply sloping than in the uniform potential vorticity case ( $\alpha=1.2$, dashed curve). 


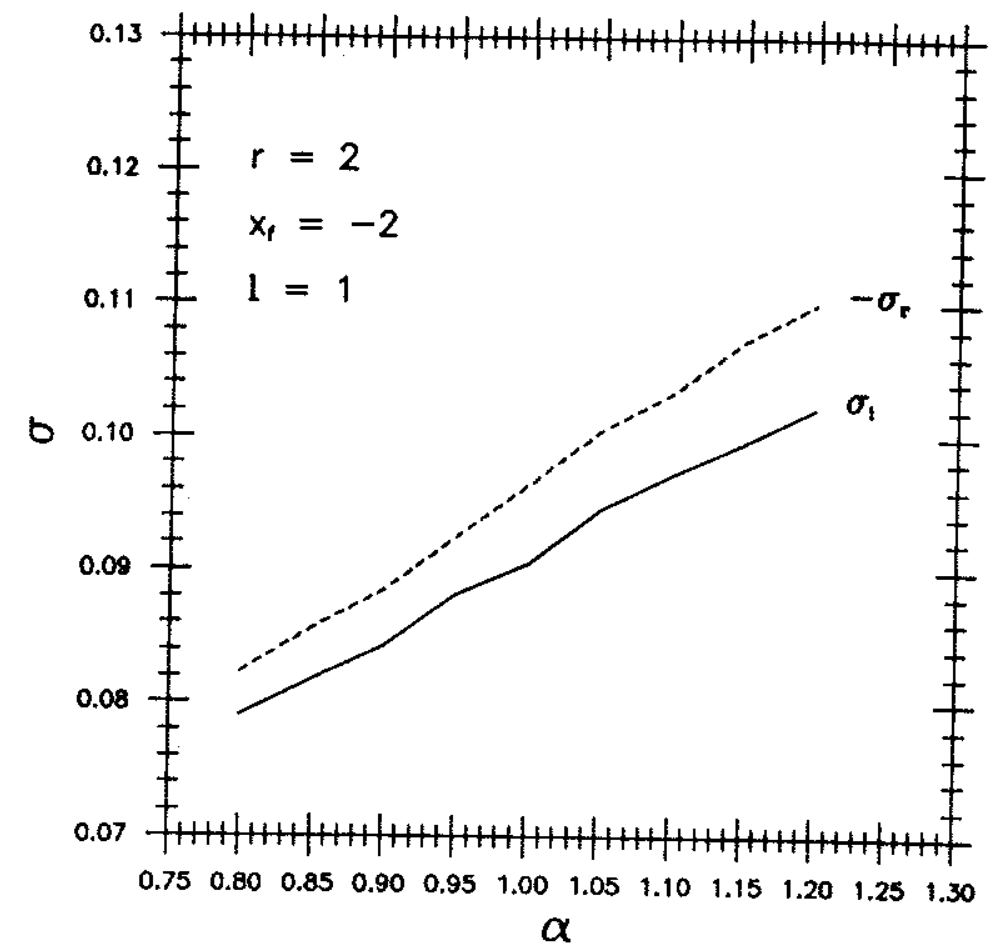

Figure 5.11: Growth rate (solid curve) and negative phase speed $\left(-c_{r}\right)$ (dashed curve) versus $\alpha$, the interface profile parameter, at the alongfront wavenumber $l=1.0$ 
between the two layers.

Numerical evaluation of the definitions of energy for the most unstable wave shows that for $\alpha<1.0$ (cross-front e-folding scale greater than the Rossby radius of deformation) the instability has $E_{2}^{\star}>0$ and $E_{1}^{*}<0$ and thus can be labelled "positive wave energy" instability. In other words, for $\alpha<1.0$ the traditional form of instability is recovered. This may be rationalized by realizing that in the limit as $\alpha$ becomes small the horizontal shear in the system decreases and (ignoring the fact that interface displacement is still large) traditional quasi-geostrophic dynamics appear more applicable. For $\alpha>1.0$, the unstable mode has $E_{2}^{\star}<0$ and $E_{1}^{\star}>0$. This "negative wave energy" instability is in the same class as those recently analyzed by Marinone and Ripa (1984).

The growth rate curve for an interface profile with $\alpha=1.2$ shows another mode of instability at high wavenumbers (Fig. 5.10). This unstable mode was not found for any value of $\alpha \leq 1.0$. The growth rate for this mode increases without bound for increasing wavenumber. As $l$ becomes large the trapping scale (which goes $\sim l^{-1}$ ) becomes shorter and shorter. Inspection of this mode reveals it to be an instability whose energy conversion is from the kinetic energy of the basic state to the disturbance. It should be noted that observations of fronts often show a great deal of mixing near the surface front. Inclusion of this process [as is done in the models of Garvine $(1983,1984)]$ may greatly influence a high wavenumber mode trapped closely to the surface front as found here. It is possible that mixing at the front could quench this mode of instability completely. The structure of $\zeta_{1}$, the surface displacement, shows a rapid phase change across the point where the phase speed of the wave equals the mean flow speed $\left(\bar{v}=c_{r}\right)$. This points to the instability being a "critical layer" phenomena. This is in fact the result of Killworth (1983), who found unstable modes in a reduced gravity model only when $\alpha$ was greater than 1.0. He obtained long wave solutions by a careful matching of solutions across a critical layer. For 
finite wavenumbers he solved the shallow water equations numerically and found a high wavenumber mode. Comparing the real part of the eigenvalue $\left(\sigma_{r}\right)$ as a function of $l$ for $l$ greater than $\sim 1.5$ calculated here to Killworth's (1983) result, shows them to be in excellent agreement. However, his results indicate that the growth rate of this mode decreases with increasing $l$ for $l$ greater than $\sim 1.5$.

To check that this mode with unbounded growth was not a result of including an active lower layer, solutions were found for the same model but with a very deep lower layer. The results for growth rate and phase speed versus alongfront wavenumber using both the geostrophic momentum equations (solid curves) and the shallow water equations (dashed curves) are shown in Fig. 5.12. The values for $c_{r}$ agree well for both sets of equations differing by less than $1 \%$ for $l \leq 5.0$. The values of $\sigma_{i}$ for the shallow water equation model decrease with increasing $l$ for $l$ greater than $\sim 2.0$. The $\sigma_{i}$ curve for the geostrophic momentum equations diverges from that of the shallow water equations at $l \sim 0.4$. For $l$ greater than $\sim 1.5$ the onelayer geostrophic momentum results for $\sigma_{i}$ exactly reproduce the values displayed in Fig. $\mathbf{5 . 1 0}$ for the two-layer model. This confirms that this mode represents a one-layer instability.

The next possibility to investigate is that the numerical solution technique may be failing to find the full eigenvalues for these high wavenumbers correctly. To check this the reduced gravity geostrophic momentum equations were solved using a numerical "shooting" technique (the code for this technique was generously provided by Glenn Ierley and Bill Young). This solution technique yielded the exact same eigenvalues as found by the global algebraic eigenvalue solution technique as displayed in Fig. 5.12. This points clearly to the failure of the geostrophic momentum approximation for this type of basic state $(\alpha>1.0)$, deep lower layers and wavenumbers greater than $\sim 0.4$. It was stated earlier that the results for $\alpha=1.0$ at all wavenumbers compared well with the solution for the shallow water equa- 


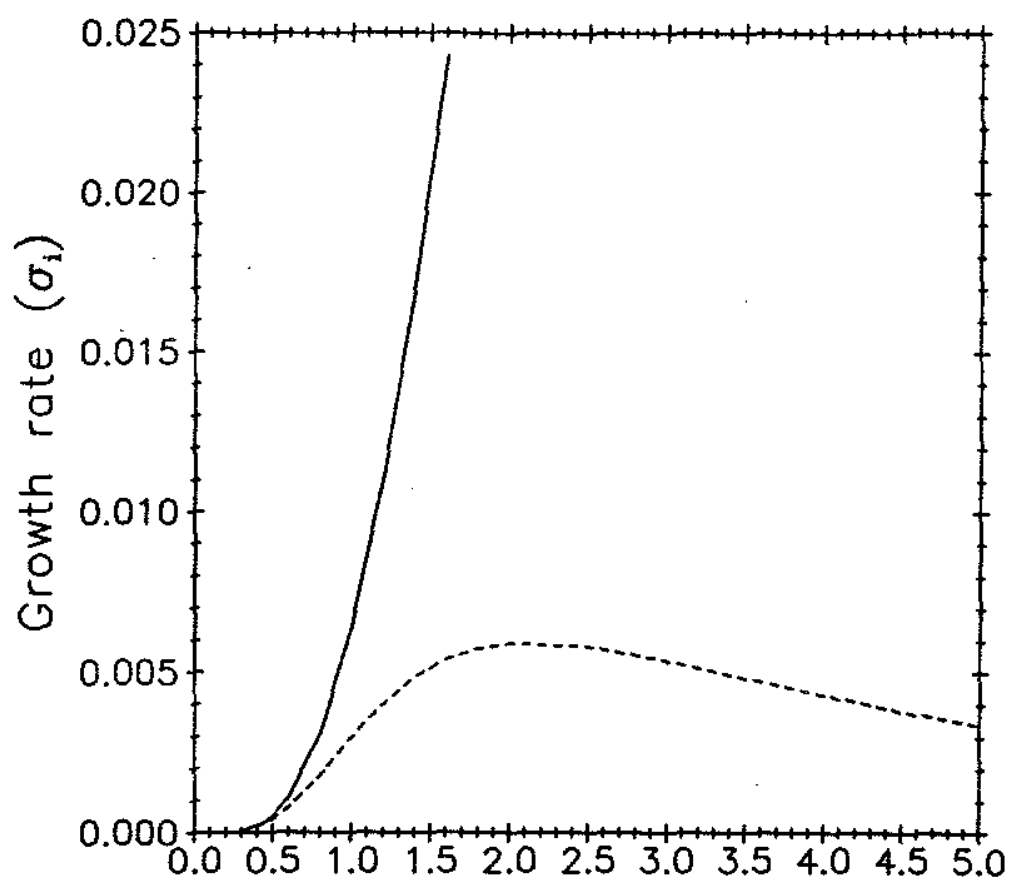

a

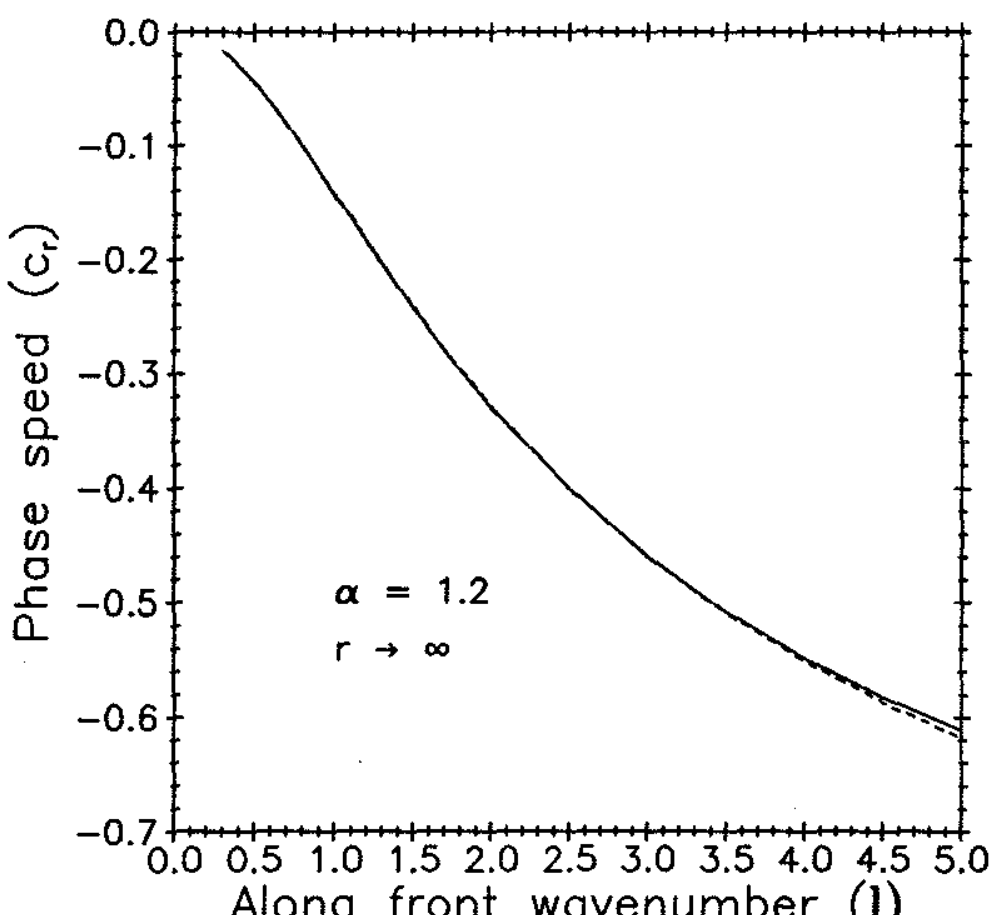

b

Figure 5.12: Comparison of (a) growth rates and (b) phase speeds for a model front with $\alpha=1.2$ obtained using the geostrophic momentum equations (solid curves) and the shallow water equations (dashed curves). 
tions found by Killworth et al. (1984). The growth curves in Fig. 5.10 at wavenumbers of $\sim 1.2$ and less for all three values of $\alpha$ appear reasonable. This implies that the geostrophic momentum approximation is finding the behavior of this baroclinic conversion mode correctly. It should also be noted that the high wavenumber mode does not violate the criteria $\sigma_{r}^{2} \ll 1.0$ until an alongfront wavenumber of $\sim 2.5$ (see Fig. 5.12).

In an effort to understand why the geostrophic momentum approximation fails for values of $\alpha>1.0$ and wavenumbers of $\sim 0.4$ and greater (for a deep lower layer), several simple reduced gravity models both with shallow water dynamics and with the geostrophic momentum approximation were attempted. Since the mode is highly trapped to the surface front a model with a linear interface ( $\bar{v}=$ constant) was considered. Analytic results were found which proved to be absolutely stable at all wavenumbers. Including horizontal shear $\left(\bar{v}_{x} \neq 0\right)$ as a modification to the local Coriolis parameter predicts instability for $\alpha>1.0$ at all $l$ with a decay in growth rate for large $l$ for both sets of equations. To determine the difference between the two sets of equations it appears necessary to include the curvature $\left(\bar{v}_{x x} \neq 0\right)$ in the mean flow. Unfortunately, this rules out analytic solutions and essentially reduces the problem to that solved numerically above.

Clearly, the geostrophic momentum approximation is failing to produce accurate results at these high wavenumbers for flows with values of the interface parameter $(\alpha)$ greater than one. This unreliable behavior at high wavenumbers is a symptom of the arbitrary truncation of the governing equations at $0\left(\epsilon^{2}\right)$ as discussed in chapter 3 . Another complicating factor may result from the fact that for $\alpha>1.0$ the absolute vorticity $\left(1+\bar{v}_{x}\right)$ vanishes in the domain of the upper layer. For $\alpha=1.0,1+\bar{v}_{x}$ vanishes at the surface front which is already a singular point of the governing equations. When $\alpha<1.0,1+\bar{v}_{x}$ does not vanish in the domain of the fluid. For $\alpha>1.0$, there may be terms neglected in the geostrophic momentum formulation which become important in a thin boundary layer about the point where $1+$ 
$\bar{v}_{x}$ vanishes. This singular structure of the governing equations is analogous to the equations governing large scale flow on a sphere where the equator is a singular point $\left(f=2 \Omega \sin \Theta\right.$ vanishes for $\left.\Theta=0^{\circ}\right)$. The dynamical balance in the equatorial region must be developed in a boundary layer structure. Though this discussion is speculative, the latter effect may contribute to the failure of the geostrophic momentum equations to accurately predict the behavior of unstable frontal waves at high wavenumbers on basic states with $\alpha>1.0$. Accurate results for this parameter range may only be obtained through consideration of the full shallow water equations (e.g. Killworth, 1983).

In conclusion, a two-layer, flat-bottom model of a coastal upwelling front with uniform basic state potential vorticity in the upper layer is unstable. The unstable mode propagates phase in the same direction as the mean flow (toward $-y$ ) and converts potential energy of the basic state flow to perturbation energy via baroclinic instability. Simultaneously, the perturbation transfers kinetic energy back to the basic state flow so as to leave the basic state flow unaltered. This results in both the mean and wave energies of the disturbance being identically zero. The unstable mode still grows with time and energy transfer occurs between the two resonating wave partners. The proximity of the front to a coastal barrier affects the growth rate of the unstable mode. The growth rate of the fastest growing wave decreases the closer the front is to the wall once that distance is less than $\sim 2-3 R$. The depth of the lower layer dramatically influences the properties of the unstable mode. Shallow lower layers increase the range of unstable wavenumbers and increase the growth rates at all wavenumbers. Increasing the vertical shear in the system (greater $\alpha$ ) increases the growth rate of the unstable mode. For $\alpha>1.0$ an unstable mode with negative wave energy is found while for $\alpha<1.0$ the unstable mode has positive wave energy. In addition, for $\alpha>1.0$ there exists a one-layer instability whose phase speed is correctly found using the geostrophic momentum approxi- 
mation, but whose growth rate is severely miscalculated. Caution should be used when modelling flows in this large wavenumber range (and with points in the flow where the absolute vorticity $1+\bar{v}_{x}$ vanishes) using the geostrophic momentum approximation.

\subsection{Inviscid, Sloping Bottom Model}

In this section the effect of sloping bottom topography on the stability of a coastal upwelling front will be investigated. All of the regions where coastal upwelling fronts are known to occur contain sloping continental shelves (e.g. Oregon, see Fig. 1.1). These shelves, specifically the region between the coastal barrier and the continental slope, may be gently sloping as off the east coast of Nova Scotia or steeply sloping as off the coast of the northwest United States. In either case, the bottom slope can be classified as strong, in the sense that the change in water depth across the shelf is large compared to the total depth. As mentioned in chapter 1, these strong bottom slopes are not allowed in quasi-geostrophic theory where the fractional change in depth must be the same order as the Rossby number which is assumed small. However, the model used here, which employs the geostrophic momentum approximation applied to the shallow water equations, will permit strongly sloping bottom topographies. The two-layer models of instability by Orlanski (1969), who used the shallow water equations, and by Mechoso and Sinton (1981), who used the quasi-geostrophic equations, both show that bottom topography sloping in the same sense as the slope of the interface stabilizes the system (i.e. reduces the growth rates of the unstable waves). The model of Orlanski (1969) did not consider the stability of a flow with a surface front in the vicinity of a strongly sloping bottom. In the previous section, the strong destabilizing effect of a shallow lower layer was demonstrated. Since coastal upwelling fronts usually form in shallow water over a sloping bottom, it is of interest to find out the net 
effect of these two opposite influences on the stability of the front.

In this section the effect of a linearly sloping bottom on the stability of a front with uniform basic state potential vorticity in the upper layer will be discussed. The total depth will take the form $H=h_{0}+s x$, where $h_{0}$ is the water depth at the coastal barrier $(x=0)$ and $s \leq 0$ is the bottom slope. As discussed in chapter 3 , the sloping bottom will join a flat-bottom region at a distance several Rossby radii offshore of the surface front. In chapter 6 , the influence of bottom topography which is an arbitrary function of $x$ and specifically chosen to model actual continental shelf profiles is presented. Since the depth of the lower layer has such a profound effect of the growth rates of unstable waves, it is desirable to try to keep the change in the depth below the surface front as small as possible while varying the bottom slope. From Figs. 5.6 and 5.7, most of the energy conversion occurs within $\sim 1$ Rossby radius of the surface front. For these reasons, the sloping bottom was pivoted about a point fixed directly beneath the surface front (see Fig. 5.13b). The values of the bottom slope given in Fig. $5.13 \mathrm{~b}$ are obtained by multiplying the actual physical bottom slope by $R / H_{1}$. This makes one unit of vertical distance equal to one unit of horizontal distance.

Before presenting detailed results, the effect of a sloping bottom on the stability of the front can be anticipated by examining the general stability criteria developed in section 4.3. For a basic state with uniform potential vorticity in the upper layer, the second inequality $(4.27 \mathrm{~b})$ is satisfied. If $\gamma$ is again chosen to be equal to -1 , which satisfies the first inequality (4.27a) at the surface front $\left(x=x_{f}\right)$ (but not at other values of $x$, see below), then to satisfy the last inequality (4.27c) the gradient of the lower layer potential vorticity must be greater than or equal to zero. This is really a requirement that the gradient of basic state potential vorticity not change sign anywhere (i.e. within either layer or between layers) in the flow. This echoes the familiar quasi-geostrophic requirements for stability, but remember now that much larger physical bottom slopes are allowed. The 


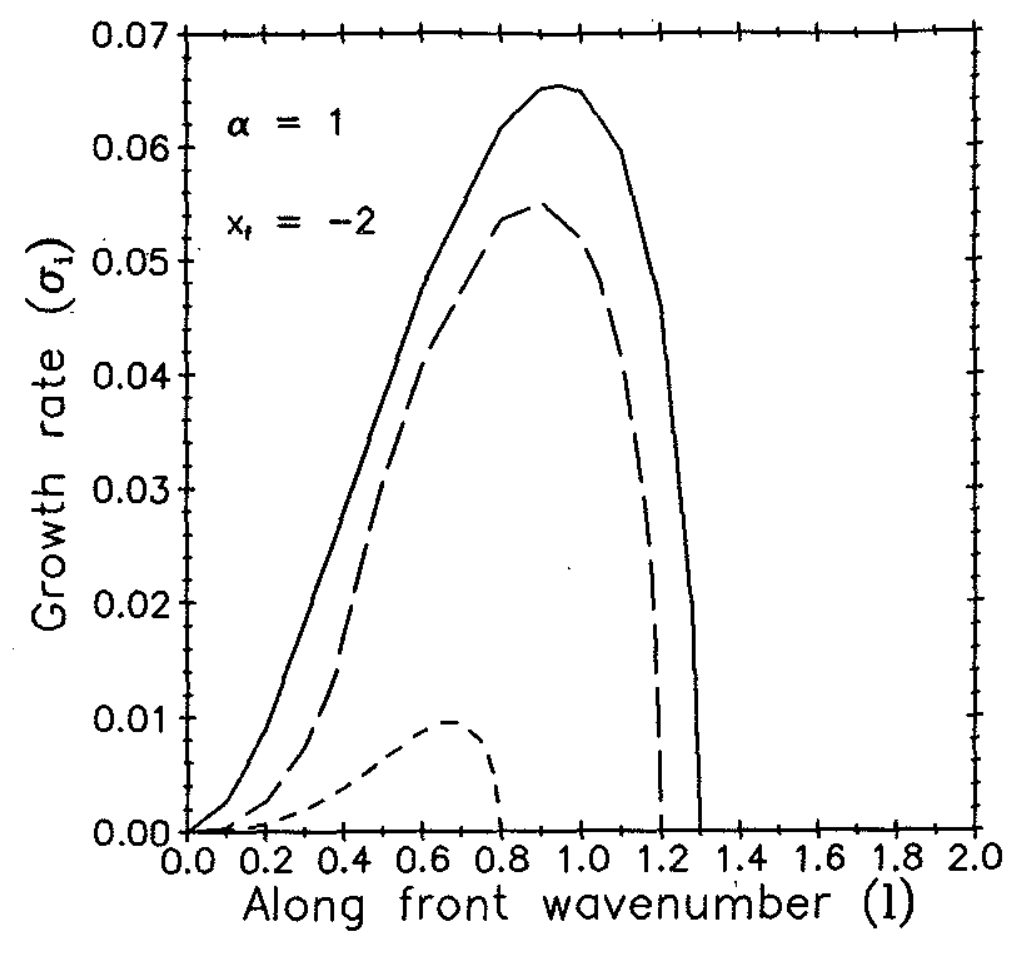

$a$

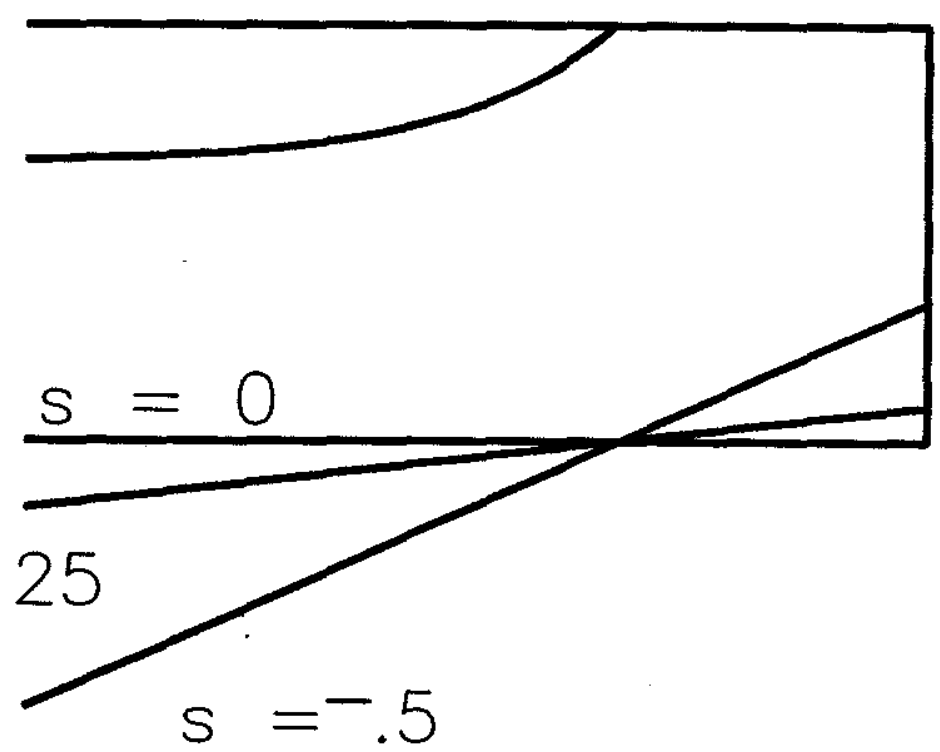

b

Figure 5.13: (a) Growth rate versus alongfront wavenumber for unstable modes present on model fronts with varying values of $s$, the slope of the linearly sloping bottom topography $(s=0$, solid curve; $s=-0.125$, long-dashed curve; $s=-0.5$, short-dashed curve) displayed in (b). 
only way to arrange this is to have the lower layer continuously thickening as $x \rightarrow-\infty$. For a linearly sloping bottom, this requires the bottom slope to be greater than the maximum slope of the interface. For the exponentially sloping interface, the maximum interfacial slope occurs at the surface front and is equal to -1 for the uniform potential vorticity front considered here. Therefore, the inequalities (4.27b) and (4.27c) will be satisfied for $s \leq-1.0$. However, the flow will still violate (4.27a) (i.e. for some range of $x \leq x_{f}$ ) so the possibility for unstable modes still exists. This mode will be analogous to the unstable mode found for the one-layer reduced gravity model with $\alpha>1.0$ (see discussion in section 4.3). Note again, that traditional quasigeostrophic stability criteria applied to this model would predict stability for $\alpha=1.0$ and $s \leq-1.0$.

Growth rate $\left(\sigma_{i}\right)$ versus alongfront wavenumber $(l)$ for models with various values of $s$ (Fig. 5.13b) are displayed in Fig. 5.13a. The solid curve for a flat-bottom model is the same as that in Fig. 5.9 for a total nondimensional depth of three $(r=3)$. The long dashed curve is for $s=-0.125$ and the short dashed curve is for $s=-0.50$. The presence of a sloping bottom decreases the growth rate of the unstable wave at all wavenumbers. The high wavenumber cutoff $\left(l_{c}\right)$ becomes smaller for increasing slope. The sloping bottom also shifts the wavenumber of the fastest growing wave $\left(l_{m}\right)$ to longer wavelengths. Note that even though the depth of the lower layer beneath the interface is increasing with decreasing $s$, the fractional decrease in the growth rate of the fastest growing wave $\left(\sigma_{i_{m}}\right)$ is more than would be expected from a depth increase alone as can be calculated from Fig. 5.9. The slope itself is stabilizing the system. The shift of the unstable modes to smaller wavenumbers occurs because the wave's cross-front velocity in the lower layer is restricted by the sloping bottom topography. In other words, the wave motion in the lower layer is forced to be more alongshore. In order to maintain a circulation in the lower layer roughly equivalent to that found in the flat-bottom case (Fig. 5.4b), the closed cell, which has 
been compressed in the cross-shelf direction due to sloping bottom topography, elongates in the alongshore direction. This leads to a larger alongfront wavelength or a smaller value of the alongfront wavenumber. For a bottom slope of $s=-1.0$ no unstable modes were found. Above, the possible existence of unstable waves even when $s \leq-1.0$ was anticipated by an examination of the general stability criteria. Violation of the necessary condition for instability is, in this case, not enough to insure the existence of an unstable mode.

Properties of the unstable modes which exist in the presence of sloping bottom topography are examined by focusing on the case $s=-0.5$. Growth rate $\left(\sigma_{i}\right)$ and alongfront phase speed $\left(c_{r}\right)$ versus alongfront wavenumber $(l)$ are plotted in Fig. 5.14. In Fig. 5.14b the unstable phase speed is denoted by a solid curve and a number of stable modes are plotted as dashed curves. As in Fig. 5.3b, the stable vorticity modes with $c_{r}<0$, except for the two modes which resonate for $l<0.8$ to produce the unstable mode, are not plotted. The phase speed of the unstable mode is less negative than in the flat-bottom case (compare Fig. 5.3b). This results because the sloping bottom induces phase propagation to $+y$, which is the same direction as topographic vorticity waves propagate. In fact, the dashed curves for $c_{r}>0$ are stable vorticity modes propagating with shallow water on their right. Only the first four stable modes with $c_{r}>0$ have been plotted. There exist many more stable waves with $c_{r}>0$ whose phase speeds decrease with increasing mode number. Among the stable vorticity modes are familiar continental shelf waves and, for this case where the interface intersects the surface, the complimentary-mode edge waves of Bane and Hsueh (1980) and Bane (1980).

The structure of the unstable mode for an alongfront wavenumber near the fastest growing wave is presented in Fig. 5.15. The upper layer structure is very similar to that for the flat-bottom mode (Fig. 5.4a). The main effect of the sloping bottom is apparent in the lower layer structure which can 


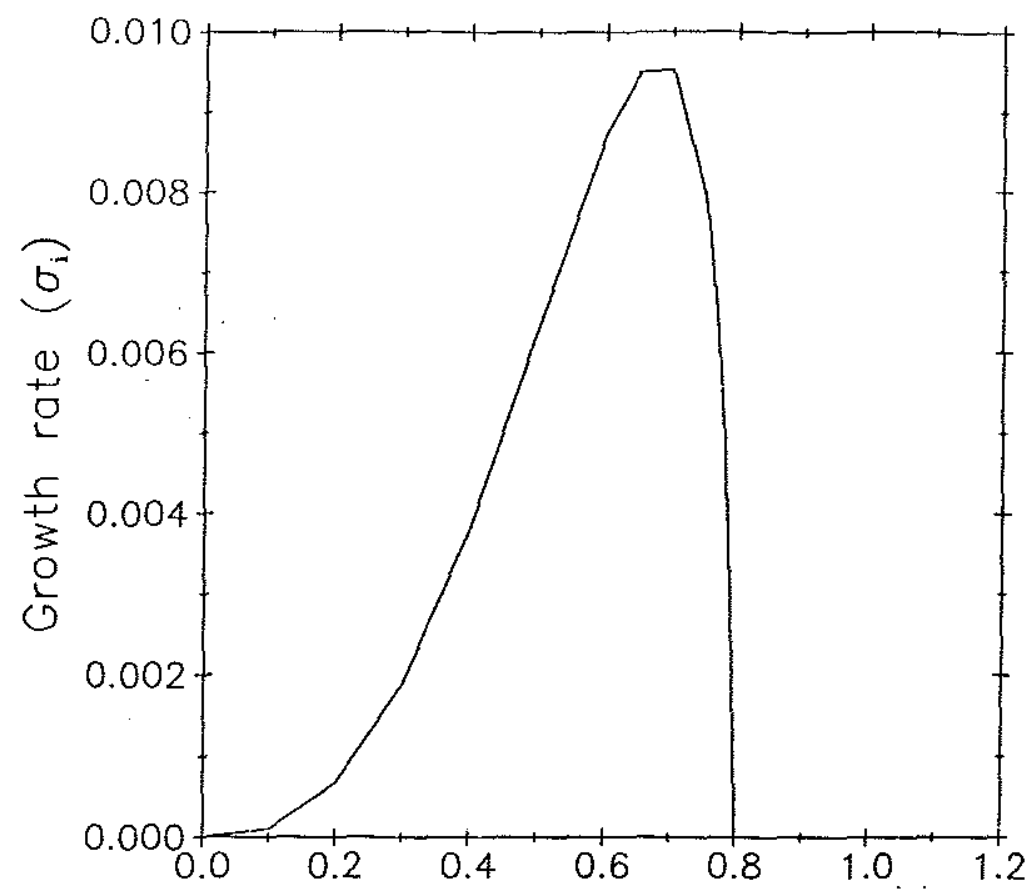

a

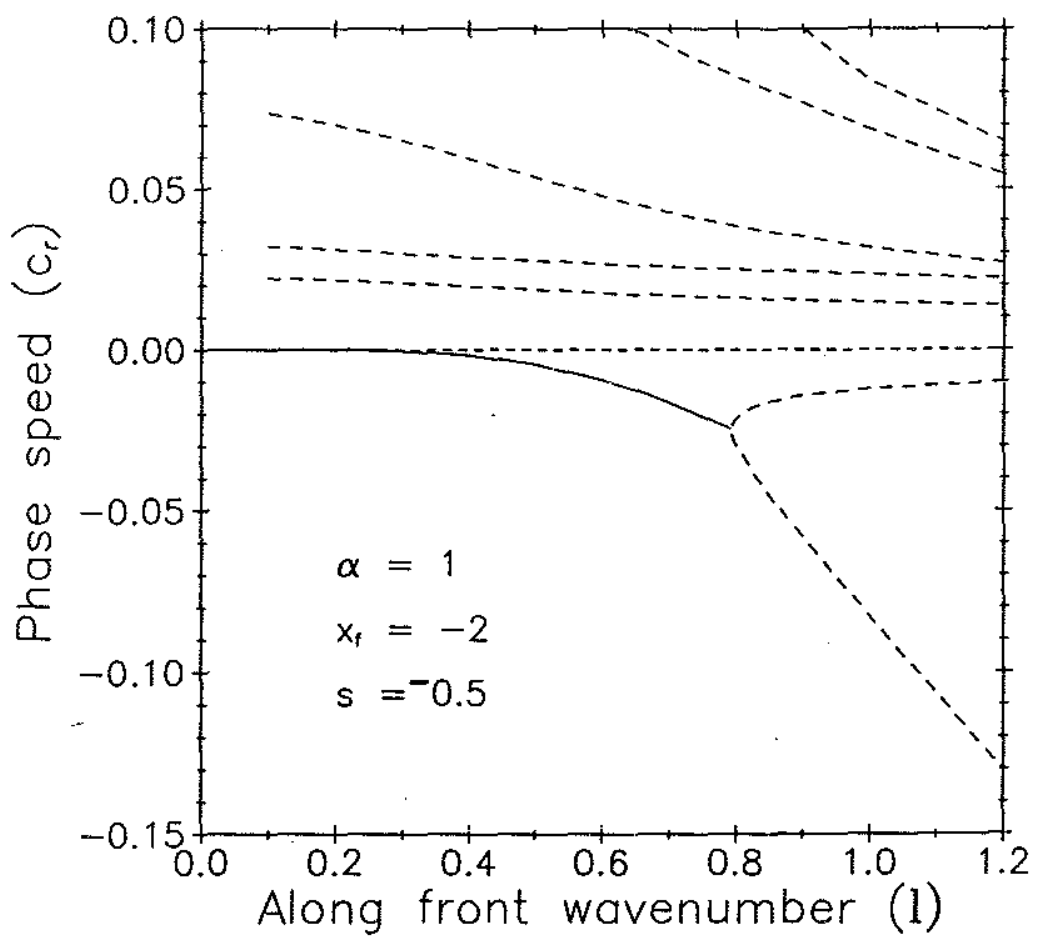

b

Figure 5.14: (a) Growth rate versus alongfront wavenumber for the unstable mode present on a model front with uniform basic state upper layer potential vorticity lying over a linearly sloping bottom $(s=-0.5)$. (b) Phase speed versus alongfront wavenumber for the unstable mode (solid curve) and a number of stable modes (dashed curves). 


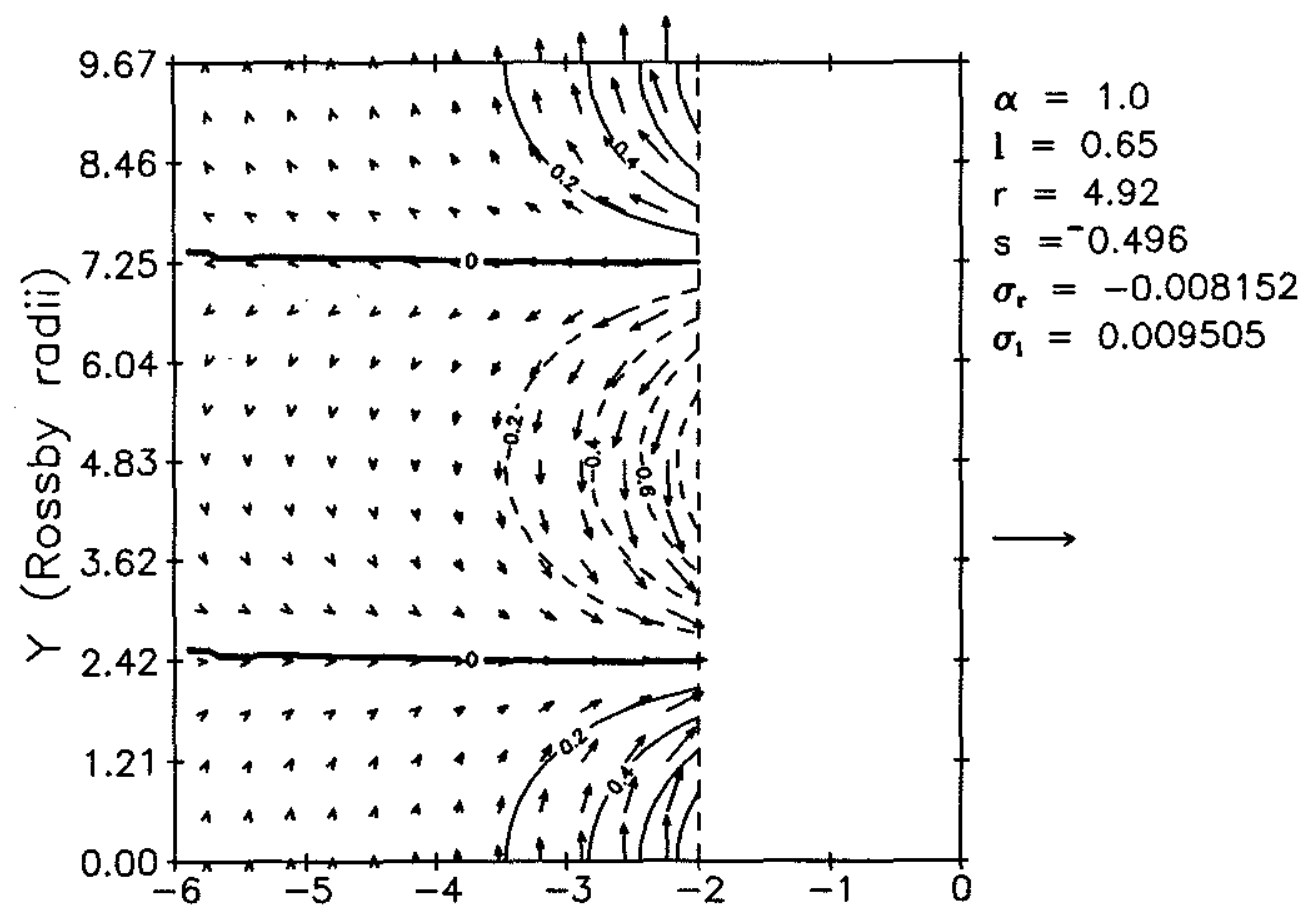

a

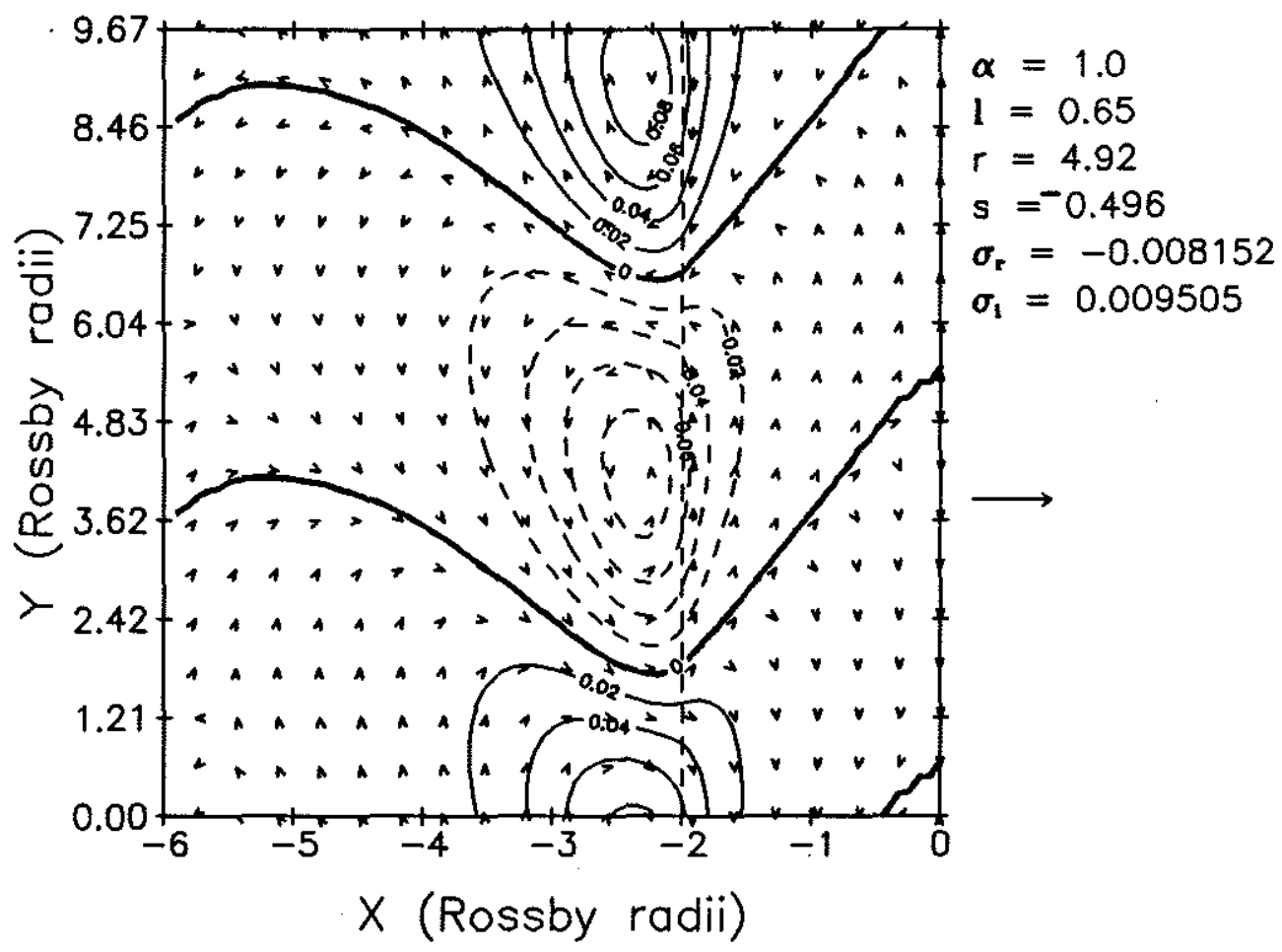

b

Figure 5.15: As in Fig. 5.4 but for an unstable mode near the fastest growing wave on a front over a linearly sloping bottom $(s=-0.5)$. 
be compared to Fig. 5.4b. As mentioned above, the unstable mode is more confined in the cross-front direction and the velocity field is more alongshore. The small growth rate for this mode is due to the upper and lower layer perturbations being more nearly in phase indicating a less effective conversion of potential energy from the basic state flow.

The transition from an unstable front over a flat bottom to a front completely stabilized by a linearly sloping bottom $(s \leq-1.0)$ is illustrated in Fig. 5.16. The phase speed $\left(c_{r}\right)$ is plotted versus alongfront wavenumber (l) for both stable (dashed curves) and unstable (solid curves) modes for a range of the values of $s$. For the flat-bottom case ( $s=0$, Fig. 5.16a) there are no stable modes with $c_{r}>0$. A number of stable modes exists for $c_{r}<0$, but only the modes with the three largest values of $\left|c_{r}\right|$ are plotted. The front is unstable over the entire range of $l$ plotted in Fig. $5.16(0.1 \leq$ $l \leq 1.2)$. With a small amount of bottom slope $(s=-0.06$, Fig. 5.16b) stable modes with $c_{r}>0$ are introduced. As in Fig. 5.14b, only the first four stable modes with $c_{r}>0$ are plotted. The phase speeds of the stable modes with $c_{r}<0$ have become less negative. The front is still unstable for wavenumbers in the range $0.1 \leq l \leq 1.2$, but now the unstable mode propagates to $+y$ for $l$ less than approximately 0.37 . The fact that these unstable modes with $c_{r}$ outside the range of the mean flow speeds (which is negative for all values of $x$ ) exist, can be rationalized by realizing that the topographic slope in this study is analogous to the effect of $\beta$ (the north-south gradient of the Coriolis parameter) in a flat-bottom model. In a quasi-geostrophic model with $\beta$, a semi-circle theorem can be established which allows unstable waves with phase speeds outside the range of the mean flow velocities (Pedlosky, 1986). Analogous possibilities are allowed in this study due to the linear bottom slope.

For a greater value of $|s|$ (Figs. 5.16c-e), the phase speeds of the stable modes with $c_{r}>0$ become greater. The phase speeds of the stable modes with $c_{r}<0$ become more positive and appear as a thick line near $c_{r}=0$ in 


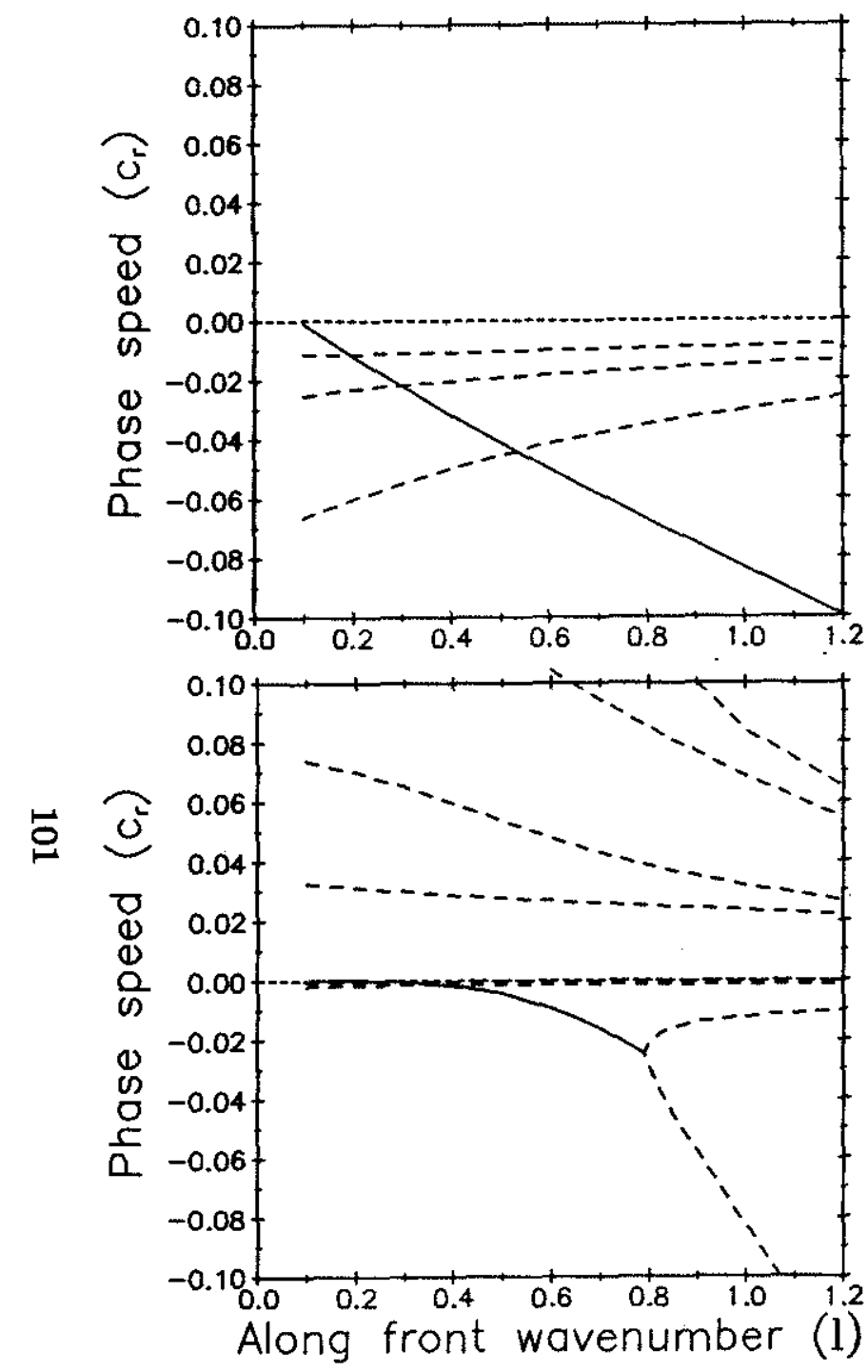

d b
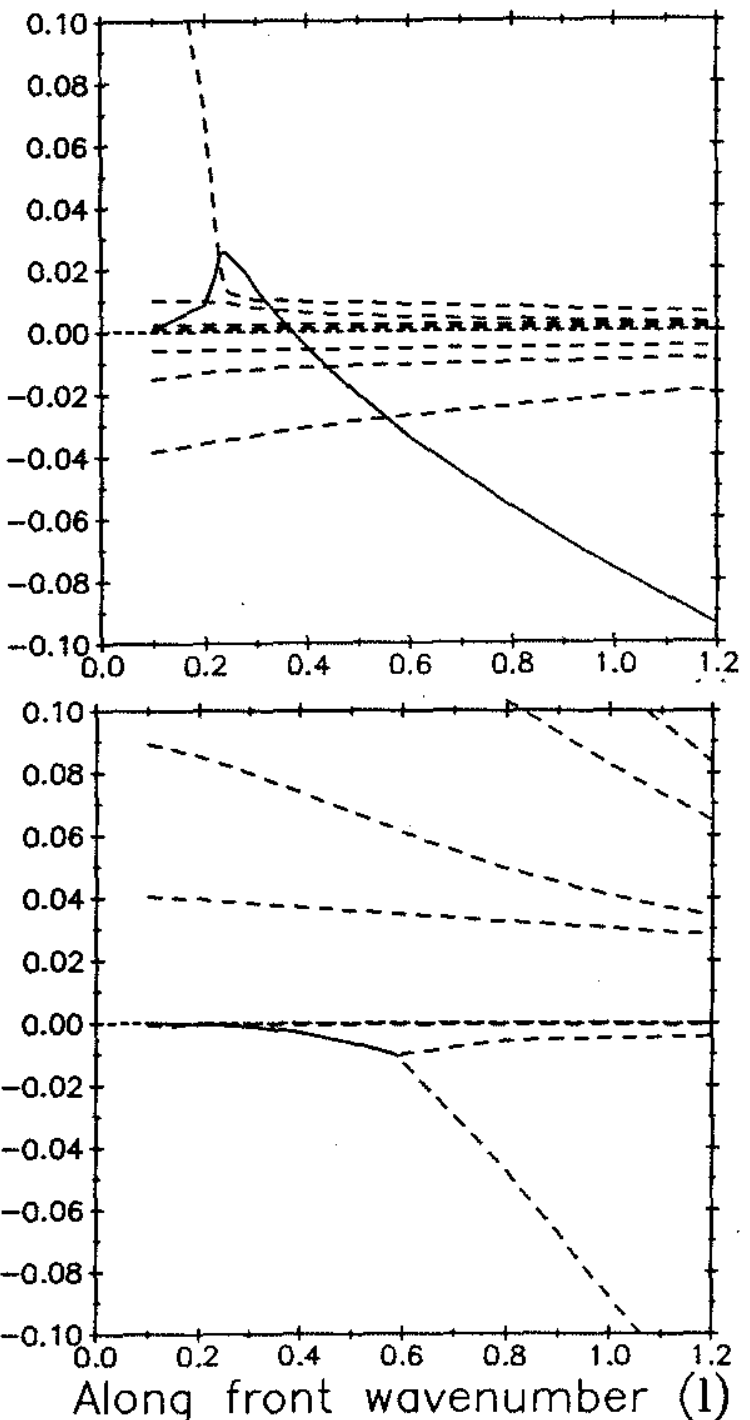

e
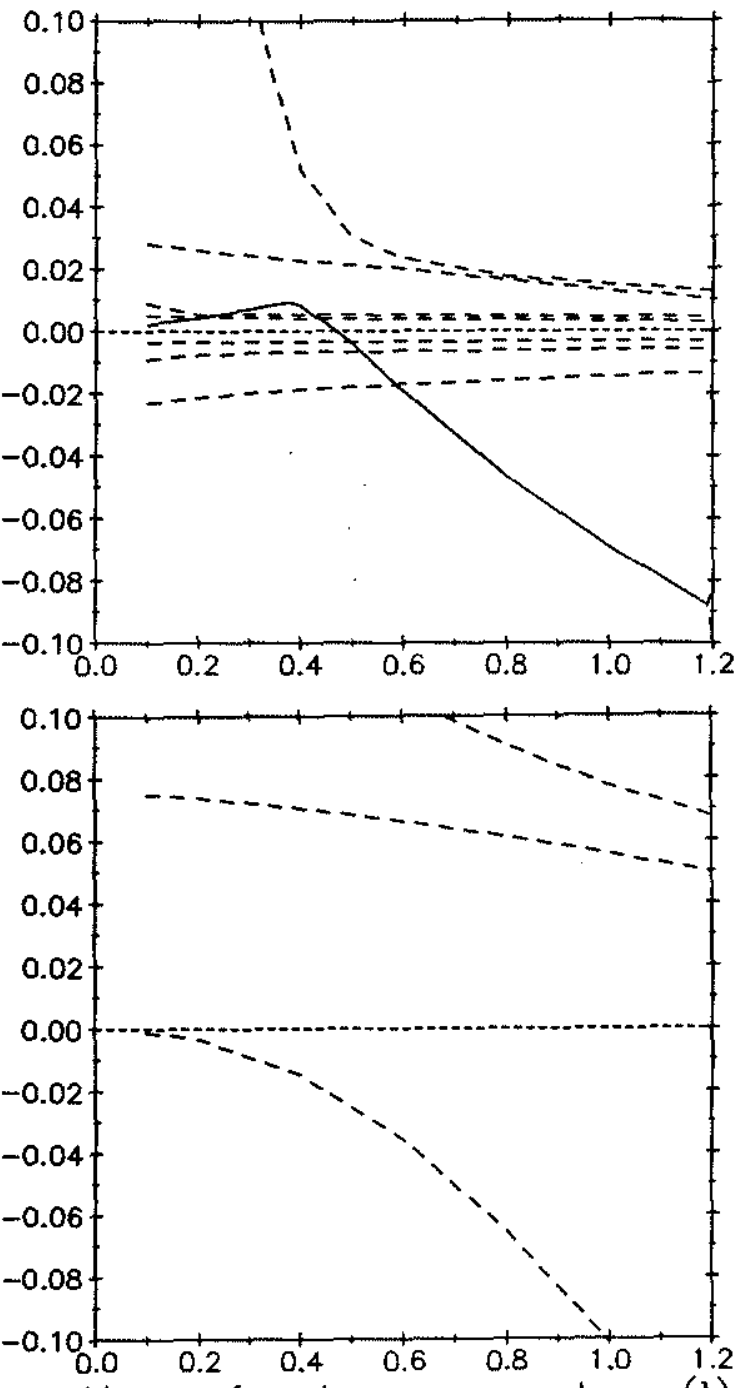

Along front wavenumber (l)

f

Figure 5.16: Phase speed versus alongfront wavenumber for the unstable mode (solid curves) and a number of stable modes (dashed curves) present for values of the scaled bottom slope parameter in the range $-1.0 \leq s \leq 0.0$. (a) $s=0$, (b) $s=-0.06$, (c) $s=-0.125$, (d) $s=-0.5$, (e) $s=-0.6$, (f) $s=-1.0$. 
Figs. 5.16d and e. Unstable waves exist for a smaller and smaller range of $l$ as $|s|$ increases. Finally, for $s=-1.0$ (Fig. 5.16f) no unstable modes are found and there exists only one stable mode with $c_{r}<0$.

Another useful set of calculations involves keeping the bottom slope constant and increasing the lower layer depth. Intuitively, one might expect the stability of a surface front over a deep lower layer not to depend on the bottom topography. Growth rate $\left(\sigma_{i}\right)$ versus alongfront wavenumber $(l)$ for a range of lower layer depths $\left[\bar{h}_{2}\left(x_{f}\right)=4,10,20\right]$ over a flat bottom (solid curves) and a sloping bottom ( $s=-\mathbf{0 . 1 2 5}$, dashed curves) are displayed in Fig. 5.17. The analogous curves for $\overline{h_{2}}\left(x_{f}\right)=3$ appear in Fig. 5.13a. The stabilizing effect of a sloping bottom is not diminished by a deep lower layer. This result is consistent with the stability theorem (4.27), since no matter how deep the lower layer is, there still exists a change in sign of the lower layer basic state potential vorticity gradient for $s>-1.0$. In the real ocean, surface-intensified features are often independent of the bottom topography. The model discussed here has a homogeneous lower layer so that bottom effects are transmitted to the entire water column. In the real continuously stratified ocean, the underlying density field may negate the effect of the bottom topography on the stability of the surface front. This omission in the simple model investigated here is discussed further in chapter 7 .

The above results indicate that a large absolute value of the bottom slope parameter $s$ may stabilize a coastal upwelling front. For realistic bottom topographies and flow fields associated with coastal upwelling fronts observed in nature, stabilization by a sloping bottom may not be realized for at least two reasons. As illustrated in Fig. 5.13a, a large absolute value of the bottom slope parameter $s$ is needed to stabilize the front. Where can these large absolute values of $s$ come from? The bottom slope $|s|$ will be large if the actual physical bottom slope is large or if the scaling factor $R / H_{1}$ is large. Since $R=\left(g^{\prime} H_{1}\right)^{1 / 2} / f$, this factor can be written as 


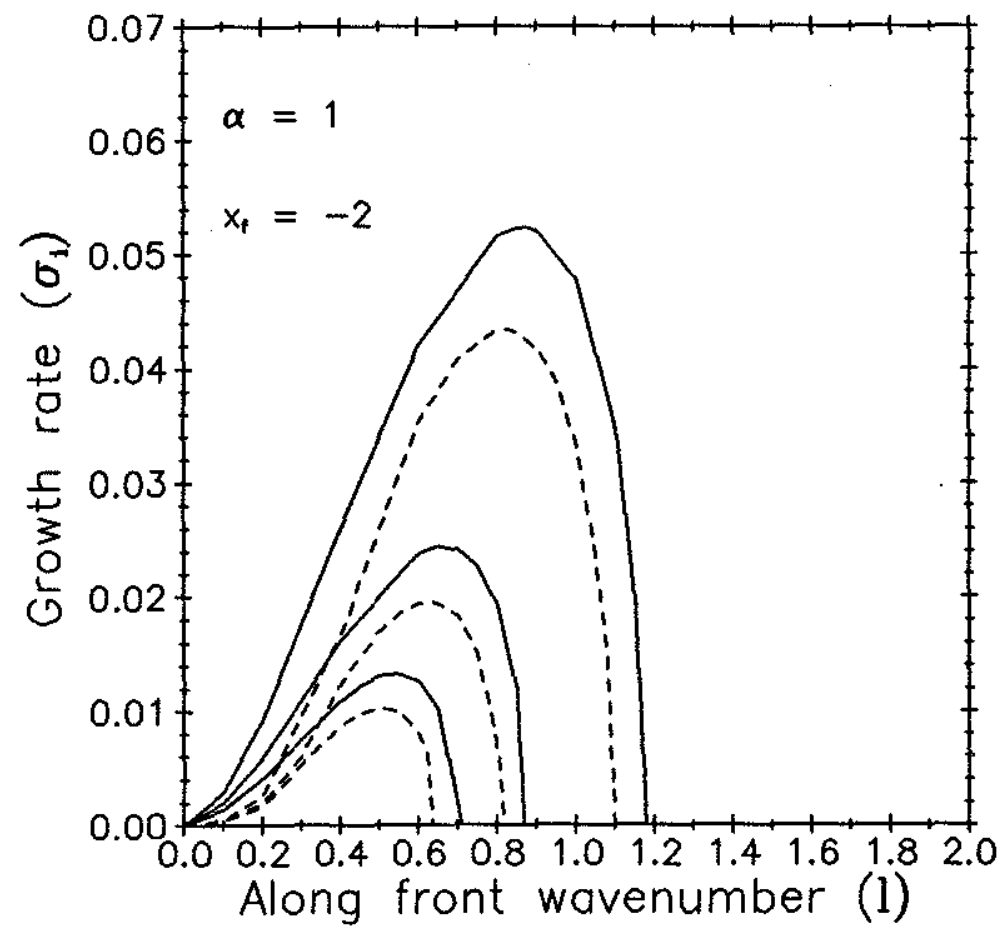

Figure 5.17: Growth rate versus alongfront wavenumber for a model front over a flat bottom (solid curves) and a linearly sloping bottom ( $s=-0.125$, dashed curves) where the three pairs of curves are for different lower layer depths. The largest growth rates are for $\overline{h_{2}}\left(x_{f}\right)=4.0$ followed by $\overline{h_{2}}\left(x_{f}\right)=10.0$ and $\overline{h_{2}}\left(x_{f}\right)=20.0$. 
$\left(g^{\prime} / H_{1}\right)^{1 / 2} / f$. Clearly, this factor will be large for a big density difference between layers or a shallow upper layer depth. Many of the coastal upwelling fronts in nature (e.g. Fig. 1.1) are of this form, so $|s|$ may be big enough to satisfy the inequality $(4.27 \mathrm{c})$, thus leaving only the possibility of the weakly growing unstable mode associated with the violation of $(4.27 \mathrm{a})$. As mentioned above, this unstable mode was not found numerically. One resolution to this is to realize that if $H_{1}$ is small then the maximum dimensional velocity (which occurs at $x=x_{f}$ ) will also be small. The basic state flow field associated with the front can then be made to violate $(4.27 \mathrm{c})$ by increasing the slope of the interface (i.e. by increasing $\alpha$ ). This will result in an increase in the maximum nondimensional upper layer velocity. However, since $H_{1}$ is small, the required maximum dimensional velocity will often not exceed speeds observed in conjunction with coastal upwelling fronts (e.g. Kosro, 1987). This discussion is intended to demonstrate one way that reasonably realistic flow fields over linearly sloping bottom topography can be potentially unstable through their violation of the inequality $(4.27 \mathrm{c})$.

Another more likely explanation for why alongfront variability is commonly observed on coastal upwelling fronts over sloping topography (i.e. the front is not stable) is that modelling the continental shelf as a constant linear slope is not very realistic. Upwelling regions of the world's oceans contain quite variable bottom topographies with both steeply and gently sloping sections. Since, in their formation process, coastal upwelling fronts migrate offshore (e.g. de Szoeke and Richman, 1984), they will pass over regions of varying bottom slope. Therefore, the front's stability will depend on the local bottom slope near (within $\sim 1$ Rossby radius) the surface front. Including a realistic bottom topography will provide a range of the values of the bottom slope parameter $s$ which contains small enough absolute values to allow for the possibility of unstable waves. A model with bottom topography which is an arbitrary function of $x$, the cross-shore direction, will 
be used in chapter 6 to verify the existence of unstable waves on realistic coastal upwelling fronts.

\subsection{Linearized Bottom Friction}

The previous results have all been for an inviscid model. Since coastal upwelling fronts form in regions of shallow water, bottom friction will certainly be present. In addition, viscous effects may be present at the sharp density interface between layers (Simpson and James, 1986). This latter process is modelled in the studies of Garvine $(1983,1984)$ by including a thin, dissipative zone adjacent to the surface front. In fact, as discussed in chapter 2, turbulent entrainment from this zone to the inviscid interior was necessary in order for Garvine (1984) to obtain spatially growing wave solutions. Since friction can be an important process in shallow coastal seas, its effect on the stability of a coastal upwelling front will be investigated in this section. First, the modifications to the governing equations and their solution due to the inclusion of dissipation will be discussed. Following this, changes in the growth rate curve due to frictional damping for one particular model configuration will be presented.

In the model investigated here, damping will be provided to the flow by linearized bottom friction. While interfacial friction may be important in the formation and evolution of a coastal upwelling front, its inclusion makes even the basic state flow difficult to solve analytically. Without a known basic state flow, the solution for the perturbations is precluded. The choice of damping by linearized bottom friction is also motivated by its importance in studies of wave motion over the continental shelf (Brink and Allen, 1978; Brink, 1982; Allen, 1984).

With linearized bottom friction, the lower layer geostrophic momentum 
equations for the perturbations (3.9) become

$$
\begin{gathered}
\zeta_{T_{y t}}+v_{2}=\zeta_{T_{x}}-\frac{\lambda}{\overline{h_{2}}} \zeta_{T_{y}}, \\
\zeta_{T_{x t}}+u_{2}=-\zeta_{T_{y}}-\frac{\lambda}{\overline{h_{2}}} \zeta_{T_{x}},
\end{gathered}
$$

where $\lambda$, the nondimensional friction coefficient, is related to the dimensional version (which has units of velocity) by

$$
\lambda_{*}=f H_{1} \lambda \text {. }
$$

Note that the friction velocities have been made geostrophic, consistent with the geostrophic momentum approximation. Substituting normal mode form for $\zeta_{T}$ [from (5.1)], the momentum equations become

$$
\begin{gathered}
\left(\sigma+\frac{i \lambda}{\overline{h_{2}}}\right) l_{\zeta_{T}+v_{2}}=\zeta_{T_{x}}, \\
-\left(i \sigma-\frac{\lambda}{\overline{h_{2}}}\right) \zeta_{T_{x}}+u_{2}=-i l \zeta_{T^{*}}
\end{gathered}
$$

Lower layer velocities will now be given by

$$
\begin{gathered}
u_{2}=-i l \zeta_{T}+\left(i \sigma-\frac{\lambda}{\overline{h_{2}}}\right) \zeta_{T_{x}}, \\
v_{2}=\zeta_{T_{x}}-l\left(\sigma+\frac{i \lambda}{\overline{h_{2}}}\right) \zeta_{T} .
\end{gathered}
$$

Finally, substituting (5.18) into the lower layer continuity equation (5.4c) results in an ordinary differential equation for $S_{T}$

$$
\zeta_{T_{x x}}+\frac{\overline{h_{2 x}}}{\overline{h_{2}}} \zeta_{T_{x}}-\left(\frac{1}{\overline{h_{2}}}+l^{2}\right) \zeta_{T}+\frac{1}{\overline{h_{2}}} \zeta_{1}=\frac{1}{\sigma}\left[-\frac{i \lambda}{\overline{h_{2}}} \zeta_{T_{x x}}+\left(l \overline{\frac{h_{2 x}}{\overline{h_{2}}}}+\frac{i \lambda l^{2}}{\overline{h_{2}}}\right) \zeta_{T}\right]
$$

The finite difference forms of this equation and the former upper layer equation (5.7a) are again combined to yield an algebraic eigenvalue problem 
of general form. The interior equations (excluding the boundary conditions which will be described below) are still linear in the eigenvalue $\sigma$, but the coefficient matrices $\underset{\approx}{A}$ and $\underset{\approx}{B}$ are now complex. Complex eigenvalue solutions to this nonhermitian system need not occur in conjugate pairs as was the case for the former inviscid system (5.8). Boundary conditions at the wall and at the offshore edge of the two-layer region are modified by the presence of bottom friction. At the wall, lower layer cross-shelf velocity (5.18a) is again required to vanish. Implementing this condition in the finite difference scheme results in terms proportional to $\sigma^{2}$. This appears to destroy the gains made by making the geostrophic momentum approximation (i.e. the algebraic eigenvalue problem was linear in the eigenvalue $\sigma$ ), but this setback will be resolved below. At the offshore edge of the domain where the interface and bottom are flat, the solution can be matched onto the two-layer frictional solution of Allen (1984). For finite depth lower layers the solution in this region will have a decay scale which depends on frequency. Only for deep lower layers will the decay scale become independent of frequency. Matching the frontal model to this solution will introduce terms proportional to $\sigma^{2}, \sigma^{3}, \sigma^{4} \ldots$ which will greatly complicate the numerical solution technique. To avoid this difficulty, a solid wall was placed at the offshore edge of the domain and normal velocity in each layer was required to vanish there. Inviscid model runs with this offshore boundary condition showed less than a $1 \%$ change in the eigenvalue compared to the open offshore boundary condition model. The finite difference forms of these boundary conditions, as mentioned above for the coastal boundary condition, introduce terms proportional to $\sigma^{2}$. The total problem, with boundary conditions, can now be written as

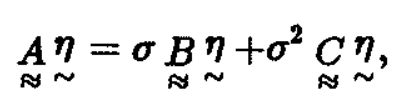

where $\underset{\sim}{\eta}$ is as defined in section 5.2. The matrices $\underset{\approx}{A}, \underset{\approx}{B}$ and $\underset{\approx}{C}$ are all complex. This algebraic eigenvalue problem which is nonlinear in the eigenvalue 
$\sigma$ can be made linear (e.g. Webster, 1987) by defining a new column vector

$$
\underset{\sim}{\xi}=\sigma \underset{\sim}{\eta}
$$

Substituting this into $(5.20)$ gives

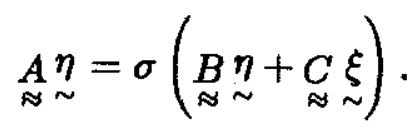

Finally, (5.21) and (5.22) can be combined and written as

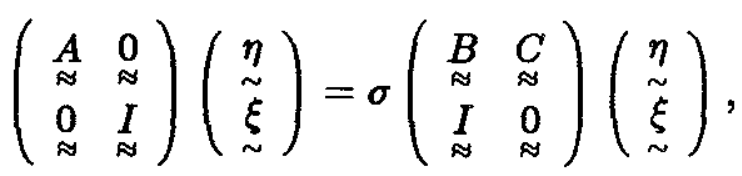

where $\underset{\approx}{I}$ is the identity matrix and $\underset{\approx}{0}$ is a null matrix. The system (5.23) is now a complex algebraic eigenvalue problem of general form which can be solved using a QZ algorithm as implemented by the IMSL routine EIGZC. The memory requirements for the problem have increased by a factor of eight (a factor of two is due to the need to store complex values in the coefficient matrices). The same procedure may be used to reduce systems with terms proportional to higher powers of the eigenvalue to problems linear in the eigenvalue. The only expense is the requirement for a large amount of computer storage space. In this study, no terms proportional to powers of $\sigma$ greater than two were retained in order to keep the problem computationally tractable.

The first model used to study the effect of bottom friction is the uniform potential vorticity flat-bottom model discussed in section 5.3. The offshore constant lower layer depth is chosen to be twice the upper layer depth $(r=3)$. The value of $\lambda$ chosen for the study was 0.2 which corresponds for an upper layer depth of $20(50) \mathrm{m}$ and mid-latitudes to a dimensional value of $0.04(0.10) \mathrm{cm} \mathrm{s}^{-1}$. Typical values for the continental shelf are from $0.015 \mathrm{~cm} \mathrm{~s}^{-1}$ to $0.08 \mathrm{~cm} \mathrm{~s}^{-1}$ (Brink et al., 1987). The model friction parameter of $0.04 \mathrm{~cm} \mathrm{~s}^{-1}$ for $H_{1}=20 \mathrm{~m}$ (roughly corresponding 
to the upwelling front in Fig. 1.1) lies within this range while the value of $0.10 \mathrm{~cm} \mathrm{~s}^{-1}$ for $H_{1}=50 \mathrm{~m}$ is probably an overestimate.

To determine what effect friction might have on the stability of the coastal upwelling front, it is useful to compare the frictional time scale (defined as the ratio of water depth to the friction parameter) to the e-folding time scales of the growing, inviscid, unstable waves. For the fastest growing wave on a uniform potential vorticity front with the offshore lower layer depth equal to twice the upper layer depth $(r=3$, growth curve displayed in Fig. 5.9), the dimensional e-folding time scale is $t_{e-f o l d} \simeq 1.8$ days. If the total depth is used, the dimensional barotropic frictional time scale is $t_{b f}=3 H_{1} / \lambda_{*} \simeq 1.7$ days. Since these time scales are comparable, it may be anticipated that bottom friction will play a major role in the stability of a coastal upwelling front. In fact, previous quasi-geostrophic instability results including damping show that friction may destabilize a system by introducing new modes of instability which were absent in the inviscid cases (Holopainen, 1961; Romea, 1977). However, since the wave motion is concentrated in the upper layer (Figs. 5.4, 5.15), the effect of bottom friction will be reduced due to the insulating presence of the lower layer. Following Allen (1984), the effective friction parameter due to stratification is obtained by multiplying $\lambda_{*}$ by $(1 / 2)\left(H_{1} / H_{2}\right)$. Since $\left(H_{1} / H_{2}\right)=1 / 2$, the effective frictional time scale will be $\sim 6.8$ days. Now the unstable wave grows on a time scale faster than the damping time scale, so the effect of friction on the wave will be weak. That is to say, friction will not significantly modify the structure of the growing wave, but can be expected to decrease its growth rate since it extracts energy from the system. These results are confirmed below.

Growth rate $\left(\sigma_{i}\right)$ versus alongfront wavenumber $(l)$ for a viscous (solid curve) and the previous inviscid (dashed curve) model are displayed in Fig. 5.18. The inviscid growth rate curve is the same as plotted in Figs. 5.9 and 5.13a. Bottom friction has decreased the growth rates for waves with 


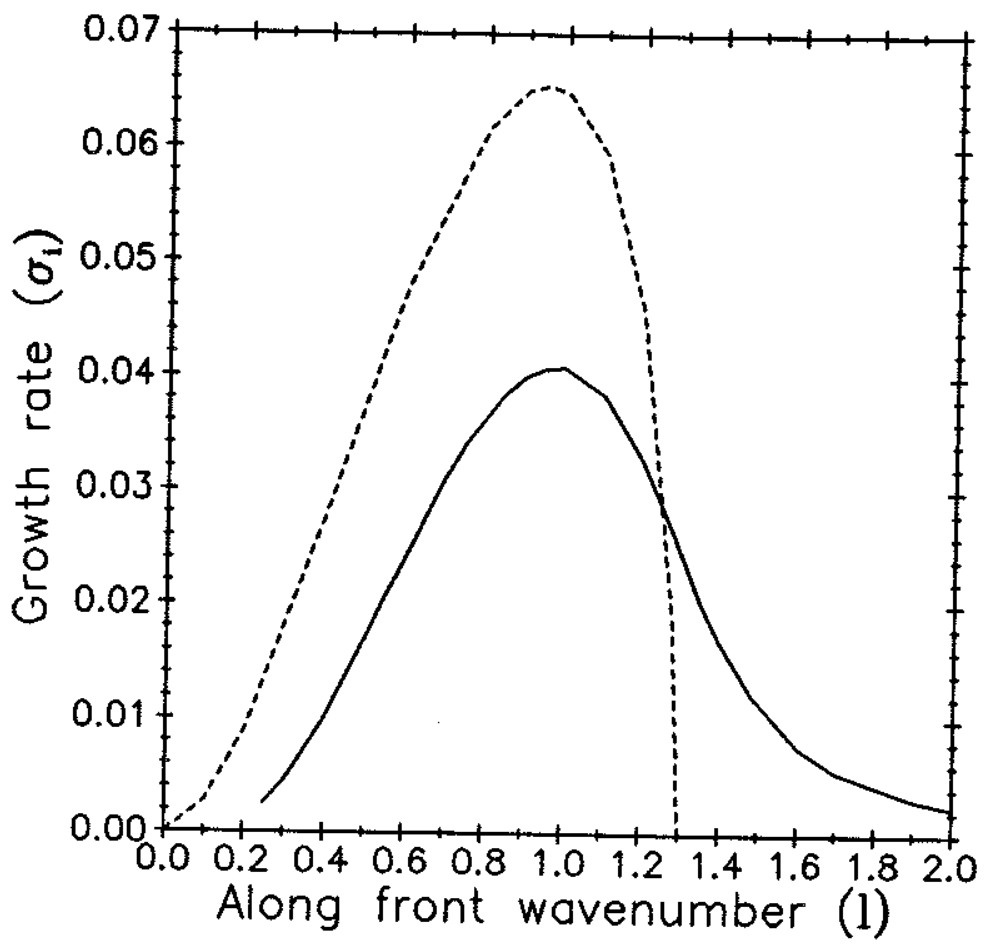

Figure 5.18: Growth rate versus alongfront wavenumber for an inviscid (dashed curve) model front and a model with linearized bottom friction $(\lambda=0.2$, solid curve). Both models have a flat bottom $(s=0, r=3)$ and uniform upper layer basic state potential vorticity $(\alpha=1.0)$. 
wavenumbers less than $\sim 1.3$. The growth rate for the fastest growing wave has been decreased by $\sim 40 \%$, but bottom friction has not significantly shifted its wavenumber. Bottom friction has also increased the unstable modes' phase speeds toward $-y$. This latter effect can be quite large, with several numerical experiments yielding increases as large as a factor of 3 or 4. At high wavenumbers, the presence of bottom friction has introduced a slowly growing unstable mode. This mode is due to the additional vorticity source provided by bottom friction. Its growth rate decreases with increasing wavenumber in a similar manner to the high wavenumber mode discovered by Killworth (1983) for a one-layer reduced gravity model. Examination of the fastest growing mode's structure in the upper layer (not shown) shows very little difference from the inviscid case. In the lower layer, the magnitude of $\zeta_{T}$ and its cross-shelf extent are decreased compared to the inviscid model. Overall, though, the structure of the viscous mode is very similar to the unstable inviscid mode.

With linearized bottom friction the conservation of wave energy (4.20) becomes

$$
\frac{\partial E_{2}^{\star}}{\partial t}=-\frac{\partial}{\partial t} \int{\bar{h}_{1}^{2}}^{2} \bar{v} Q_{1_{z}} \frac{\eta_{1}^{2}}{2} d A-\lambda \int\left(\zeta_{T_{z}}^{2}+\zeta_{T_{y}}^{2}\right) d A
$$

Since $Q_{1_{x}}$ is zero for this particular basic state flow, the wave energy at all wavenumbers will be negative. As mentioned above, a high wavenumber mode exists in the presence of bottom friction, but is absent in the inviscid case. For large wavenumbers friction is destabilizing the system. The existence of this high wavenumber mode may be rationalized be recalling the comments made in section 4.2 about the potential for instability due to resonance between a stable wave and bottom friction. For the unstable alliance to occur, the wave partner must have negative disturbance (wave $E_{2}^{\star}$ plus mean $E_{1}^{\star}$ ) energy so it can lose energy to dissipation. The resulting unstable wave has zero net disturbance energy as the wave transfers disturbance energy to dissipation. The high wavenumber mode found here is 
an example of this process which is described further by Cairns (1979).

The high wavenumber mode found here in the presence of friction can be compared to the high wavenumber mode found in the inviscid model with $\alpha>1.0$ (see section 5.3 and Killworth, 1983). Both modes have negative wave energy. For the inviscid model with $\alpha>1.0$, the vorticity source for the perturbations is the nonzero basic state potential vorticity gradient in the upper layer. In the viscous case, bottom friction provides the vorticity source for the perturbations. In either case the high wavenumber modes are trapped closely to the front and have small growth rates. As mentioned in section 5.3, these modes may not be observed in nature not only because they grow slowly, but also because cross-frontal mixing near the surface front may quench them completely.

Results for a model with a flat-bottom and $\alpha \neq 1.0$ are qualitatively similar to those discussed here for $\alpha=1.0$. Specifically, the growth rate of the fastest growing wave for all $\alpha$ is decreased and a slowly growing, high wavenumber mode exists in the presence of friction for $\alpha<1.0$. The high wavenumber mode for $\alpha=1.2$, whose growth rate is miscalculated using the geostrophic momentum approximation (see section 5.3 ), is essentially unaffected by the presence of bottom friction.

The primary effects of bottom friction on the unstable modes discussed above is to decrease the fastest growing mode's growth rate and increase its phase speed in the direction of the mean flow. This results because the frictional damping time scale is much longer than the e-folding time scale of the growing wave. When these time scales are more comparable, friction may destabilize the system (Holopainen, 1961; Romea, 1977). To illustrate this effect, the model was run with a uniform basic state potential vorticity flow in the upper layer $(\alpha=1.0)$ and a sloping bottom such that $s=-1.0$. As shown in section 5.4, the inviscid version of this model is absolutely stable. When bottom friction is added to the system, a weakly growing $\left[\sigma_{i_{m}} \simeq 6.0 \times 10^{-3}\right.$ for a model with $\overline{h_{2}}\left(x_{f}\right)=3.0$; compare to growth rates 
in Fig. 5.18] unstable mode appears. This destabilization by friction in the presence of topography is analogous to the quasi-geostrophic models with the $\beta$ effect and bottom friction (Holopainen, 1961; Romea, 1977).

The destabilization of the flow through the introduction of viscosity is a counterintuitive result. This effect can be rationalized in several ways. One simple explanation is that bottom friction breaks the connection between the interior flow and the bottom slope. That is, the strong constraint for flow along isobaths is broken by the presence of bottom friction. A second explanation relies on the fact that friction introduces a phase shift in the disturbance. This phase shift allows the wave to release energy from the basic state flow. It can be shown that the gain in energy is larger than the loss of energy to dissipation so the disturbance amplifies. The final interpretation, as mentioned earlier, involves an alliance between dissipation and a stable wave with negative disturbance energy. The stable wave propagating to $-y$ in the inviscid model with $s=-1.0$ (see Fig. 5.16f) has negative disturbance energy. This mode grows as it transfers disturbance energy to dissipation in order to maintain zero net disturbance energy.

In conclusion, bottom friction, which is known to be an important process in the coastal ocean, is not expected to completely quench the unstable waves present on a coastal upwelling front. This is mainly due to the fact that the motion of the unstable wave is concentrated in the upper layer and is thus insulated from the damping effect of bottom friction by the presence of the lower layer. However, bottom friction does provide a sink of energy to the system so a decrease in the growth rate of the fastest growing mode can be expected. For a value of the friction parameter in the range of observed values and for a constant offshore layer depth equal to twice the upper layer depth, this decrease in growth rate is on the order of $40 \%$. For weaker friction or deeper lower layers this effect will be less. Finally, an example of destabilization by bottom friction is provided by a model with a steeply sloping bottom $(s=-1.0)$. The unstable mode in this case has a 
very small growth rate.

\subsection{Summary}

In this chapter the existence of unstable waves on model upwelling fronts has been demonstrated. For a front next to a coastal wall over a flat bottom, the fastest growing wave gains energy from the basic state potential energy via baroclinic instability. Numerical confirmation of the conservation statements set forth in chapter 4 was obtained. In particular, a negative contribution to the wave energy form the cross-term $-\bar{v} v_{1} \zeta_{2}$ was found. For a front with uniform basic state potential vorticity in the upper layer, this negative contribution exactly balances the positive sum of the kinetic and potential energies to make the wave energy identically zero.

A decrease in the fastest growing wave's growth rate was obtained when the surface front is moved closer than $\sim 2 R$ to the coastal barrier. Deep lower layers decrease the growth rate for all values of alongfront wavenumber due to the decreased overall vertical shear. Increasing the steepness of the interface by increasing the parameter $\alpha$ results in a gain in the fastest growing wave's growth rate. For an interface profile steeper than a front with uniform basic state upper layer potential vorticity, a high wavenumber mode was found whose phase speed is correctly found with the geostrophic momentum equations, but whose growth rate is badly miscalculated. A linearly sloping bottom decreases the growth rate for all values of the alongfront wavenumber. A large enough bottom slope can completely stabilize the front by removing the change in sign in the potential vorticity gradient of the lower layer. However, realistic continental shelf topographies contain a wide range of bottom slopes and are not expected to completely stabilize a coastal upwelling front. Finally, linearized bottom friction decreases the growth rates of the unstable waves since it extracts energy from the system. For $\alpha \leq 1.0$, a high wavenumber unstable mode 
exists because of the presence of bottom friction. In general, this mode has small growth rates. Bottom friction may also destabilize a flow which is otherwise stable in the absence of dissipation. The resulting unstable mode has a very small growth rate. In the next chapter, the predictions for the properties of the fastest growing wave in a realistic stability model will be compared to observations of alongfront variability on coastal upwelling fronts. 


\section{Chapter 6}

\section{Comparison with Observations}

\subsection{Introduction}

The results from the simple stability analysis presented in the previous chapters show that unstable waves do exist on model coastal upwelling fronts. The alongfront wavelength, e-folding time and propagation speed of the fastest growing wave depends on various properties of the basic state flow and model geometry. Observations of upwelling fronts in many regions of the world's coastal oceans show alongfront variability in the offshore position of the surface front. As noted in chapter 1, these alongfront meanders often appear wavelike and can extend alongshore over many repeated wavelengths. Observations also yield evidence for temporal growth in the size of these alongfront disturbances. The purpose of this chapter is twofold. First, observations of coastal upwelling fronts from several regions of the world's oceans will be examined for evidence of unstable frontal waves. Second, the scales of the observed alongfront variability will be compared to the properties predicted by the simple stability model presented in the previous chapters.

Sea surface temperature (SST) maps from almost any region of active coastal upwelling show meanders in the surface temperature front. The discussion presented here is not intended to be a comprehensive worldwide 
survey of frontal variability nor is it meant to be a detailed examination of the physical dynamics of coastal upwelling. References to studies of this type are included in chapter 2. Rather, case studies from selected upwelling regions will be analyzed concentrating on the properties and evolution of wavelike perturbations in the coastal upwelling front. These regions are off the coasts of Oregon, Nova Scotia and southwest Africa.

As mentioned in section 5.4, the bottom topography of the continental margin contains regions of varying bottom slope. In order to include realistic bottom profiles, the stability model will be modified to include bottom topography which is an arbitrary function of $x$, the cross-shelf coordinate. This more physically realistic model with the appropriate input parameters will then be used to determine the properties of the fastest growing wave. According to the linear theory developed here, this is the mode which will be observed in nature as it grows most rapidly from a small initial disturbance containing many wave components. The model presented here does not determine the unstable waves' properties at finite amplitude but, nevertheless, provides estimates which can be compared to observed frontal variability. In fact, reasonable agreement between the properties of the predicted unstable waves and observed frontal variability suggests that the linear model predictions may hold at finite amplitude.

\subsection{Oregon}

The coastal ocean off Oregon is an area of active upwelling during the summer when winds become predominantly upwelling-favorable (blowing with the coast on the left)(e.g. Curtin, 1979). An example of a coastal upwelling front in this region was presented in chapter 1 (Figs. 1.1, 1.2). Two time periods during which almost daily aircraft SST maps are available (O'Brien et al., 1974) will be examined for the presence of unstable frontal waves. The first time period is July 12-15 after the peak of a strong (wind 
stress greater than $4 \mathrm{dyn} \mathrm{cm}^{-2}$ ) upwelling-favorable wind event (Fig. 6.1). A SST map from the middle of this period (July 14) was shown in Fig. 1.2 and is repeated in Fig. 6.2, which also contains maps earlier (July 12) and later (July 15) in time. On July 12 (Fig. 6.2a), active upwelling is indicated by the presence of cold water near the coast. At this time the surface front is not very sharp. That is, the temperature change between inshore, cold, upwelled water and the warmer, offshore waters occupies a region at least $35 \mathrm{~km}$ wide. Note the tendency for the surface isotherms to run parallel to the coast. However, they are not absolutely parallel to the coast or the bottom topography. An alongfront meander exists in the surface isotherms (e.g. the $49^{\circ}$ contour).

On July 14 the wind stress has decreased (Fig. 6.1) as the upwellingfavorable wind event finishes. The SST map (Fig. 6.2b) shows a sharp, pronounced surface front $\sim 20 \mathrm{~km}$ offshore. An alongfront meander pattern, which appears wavelike and extends alongshore over approximately two wavelengths, is clearly evident. The northern part of the disturbance has a larger wavelength than the southern part. A quantitative estimate of the wave properties is presented below. Tracing isotherm position (e.g. using the $49^{\circ}$ isotherm) from the SST map of July 12 to July 14, shows that the alongfront meander has essentially grown (larger cross-front peak-to-peak amplitude) without the whole pattern moving much alongshore. Growth in time of a meander pattern does not alone justify the interpretation of the phenomenon in terms of an instability process. For instance, stable wind-driven motions will change in amplitude as the forcing changes in time. However, growth in time and propagation of the meander pattern in the same direction as the basic state flow (which will be established below for the case studies from each of three geographic locations) do support the idea that the observed wavelike perturbations result from an instability process.

The wavelike pattern evident in Fig. $6.2 \mathrm{~b}$ does not have a symmetric 

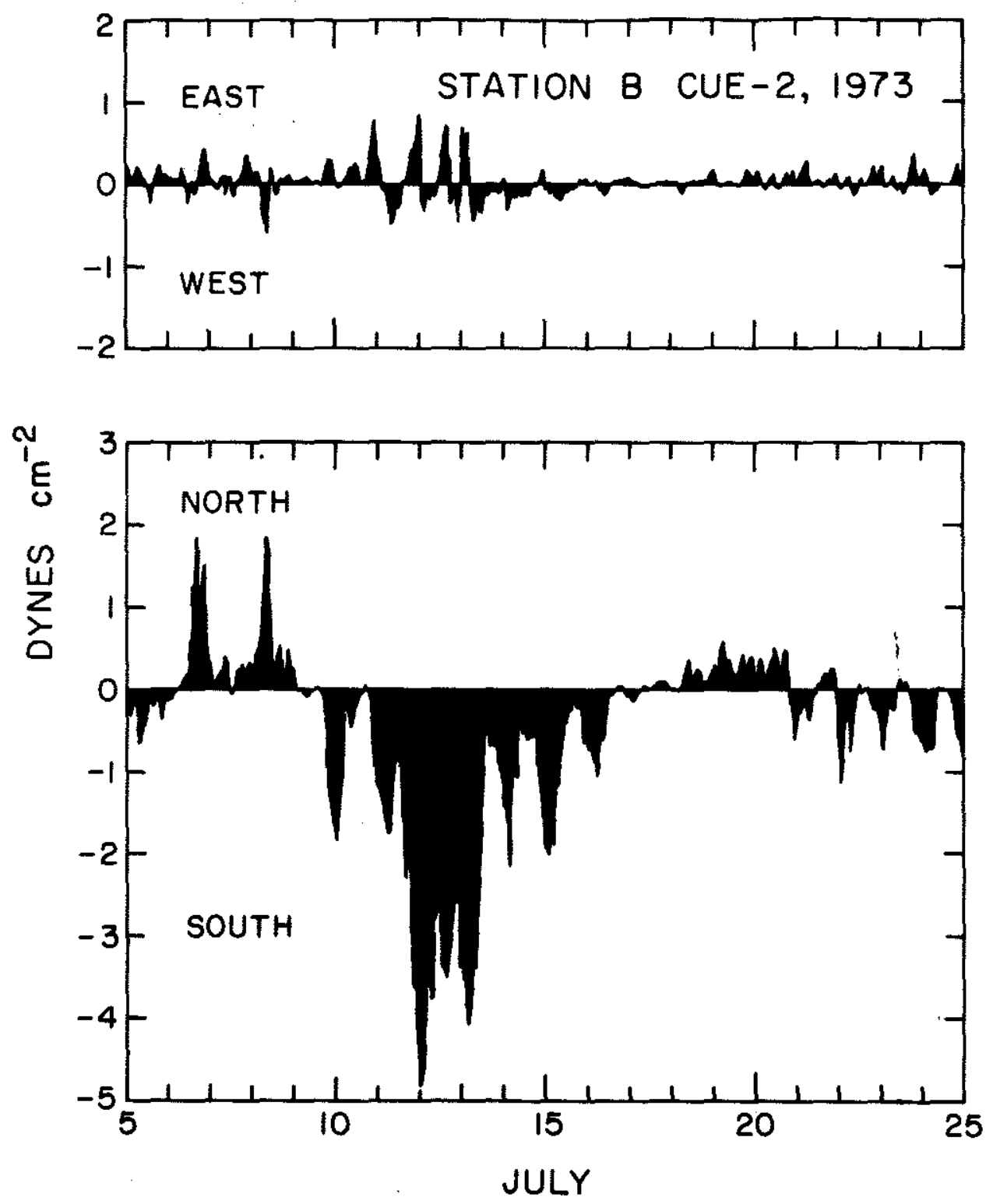

Figure 6.1: Wind stress measured off Oregon during summer, 1973. The lower panel shows alongshore wind stress where negative (equatorward) values represent upwelling favorable winds. From Halpern, 1976. 


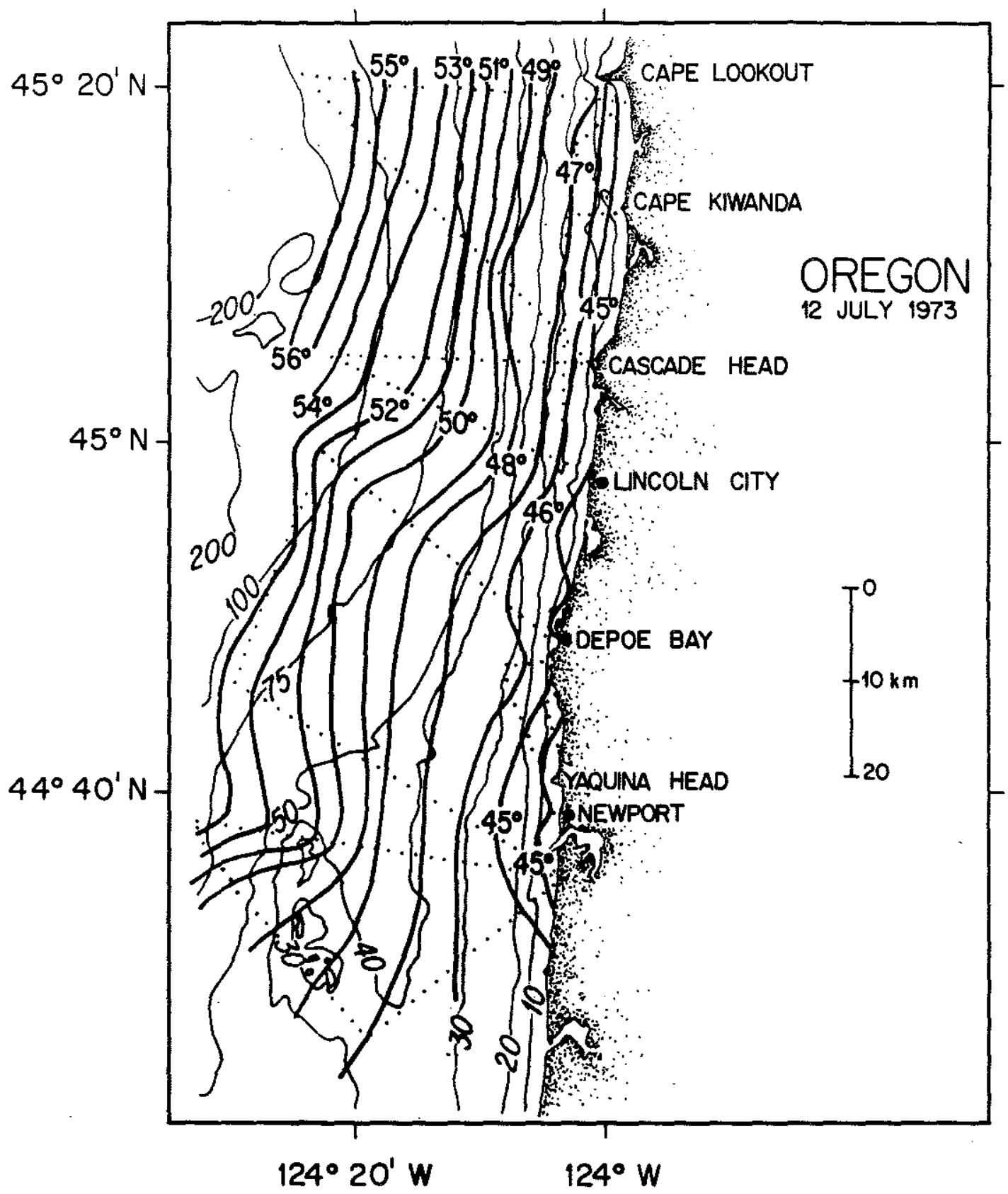

Figure 6.2: Sea surface temperature (in degrees Fahrenheit) from aircraft surveys off the coast of Oregon on (a) July 12, (b) July 14 and (c) July 15, 1973. Flight path is shown as a dotted line. Depth contours are in fathoms $(1 \mathrm{fm}=1.8 \mathrm{~m})$. From O'Brien et al., 1974 . 


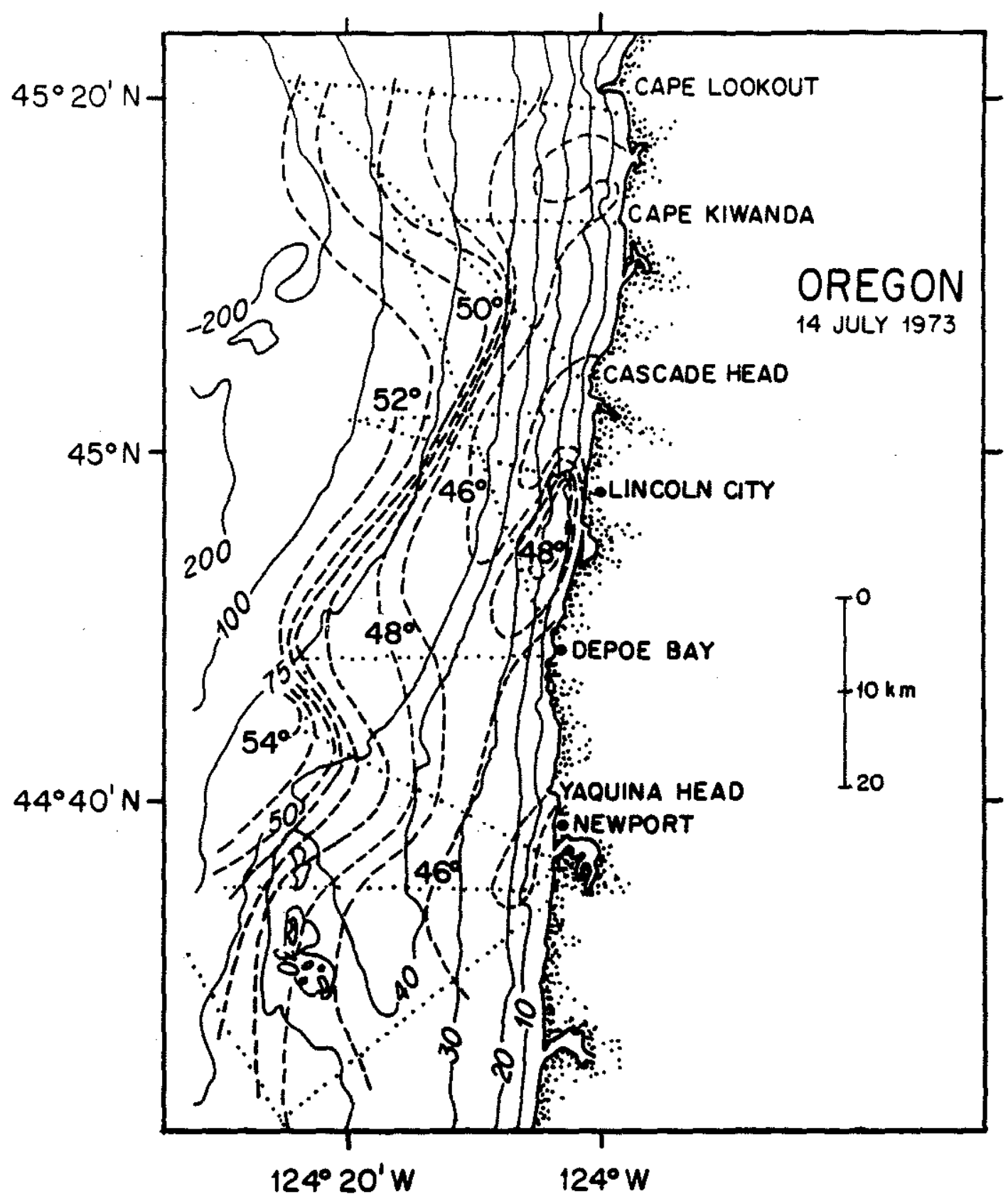

b 


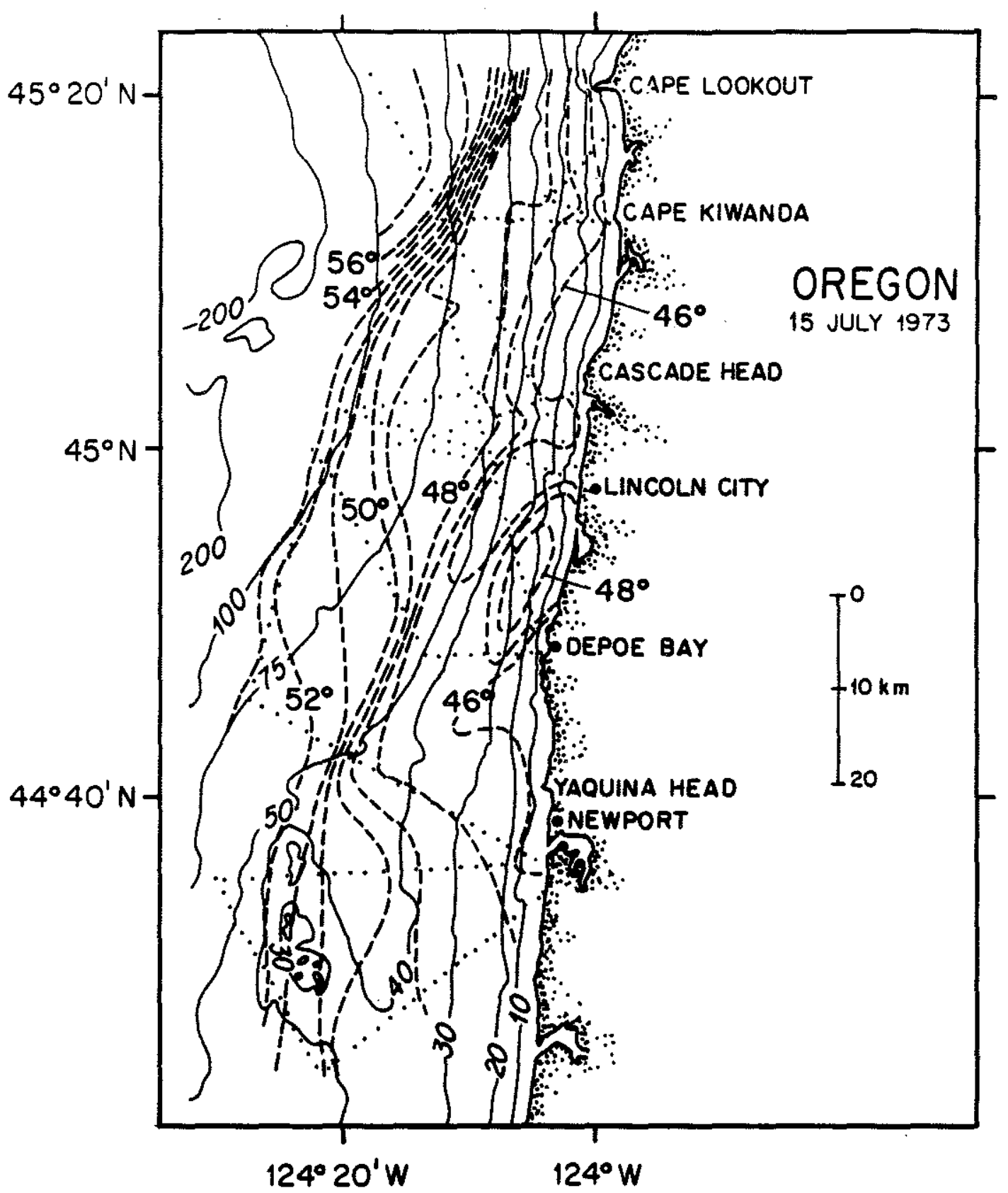

c 
sinusoidal shape. There is clear evidence that the surface front's position moves more quickly from offshore to onshore than from onshore to offshore. In other words, the wave is steeper downstream of regions where the upper layer is shallow. This type of pattern is consistent with the alongfront advection of layer height implied by the negative contribution of the term $-\bar{v} v_{1} \zeta_{2}$ to the wave energy discussed in chapter 4. As displayed in Fig. 4.1, this contribution implies that regions where the upper layer is shallow travel more quickly downstream than regions with thick upper layers. A planview of this pattern as obtained from a conceptual model of wave steepening is displayed in Fig. 6.3. Two comments can be made on the wave steepening effect. The first is that wave steepening is a nonlinear effect and the unstable wave motions modelled here are strictly linear. However, wave steepening is consistent with the predicted linear solution and does appear in observations of alongfront variability. The second point is that the wave steepening observed here is in the opposite sense to that predicted for stable waves over sloping bottom topography in the presence of a surface front. Gill and Schumann (1979) show that for a surface front over a linearly sloping bottom, only stable waves with poleward speeds less than the mean flow speed exist. They further note that for this supercritical flow, the inshore part of the wave moves more quickly to $-y$ than the offshore part. This leads to wave steepening downstream of the region where the upper layer is deep. Therefore, the observations presented here are more consistent with unstable frontal waves than with stable topographic waves in the presence of a surface front.

Compression and rarefaction of surface isotherms within the frontal zone is apparent in Fig. 6.2b. This pattern may be due to the presence of a shallow bottom and a coastal barrier on one side of the front. On the inshore side of the front, the bottom and coast restrict the cross-shelf movement of the surface isotherms which leads to their compression. On the offshore side, the peaks grow without restriction so no compression occurs and the 


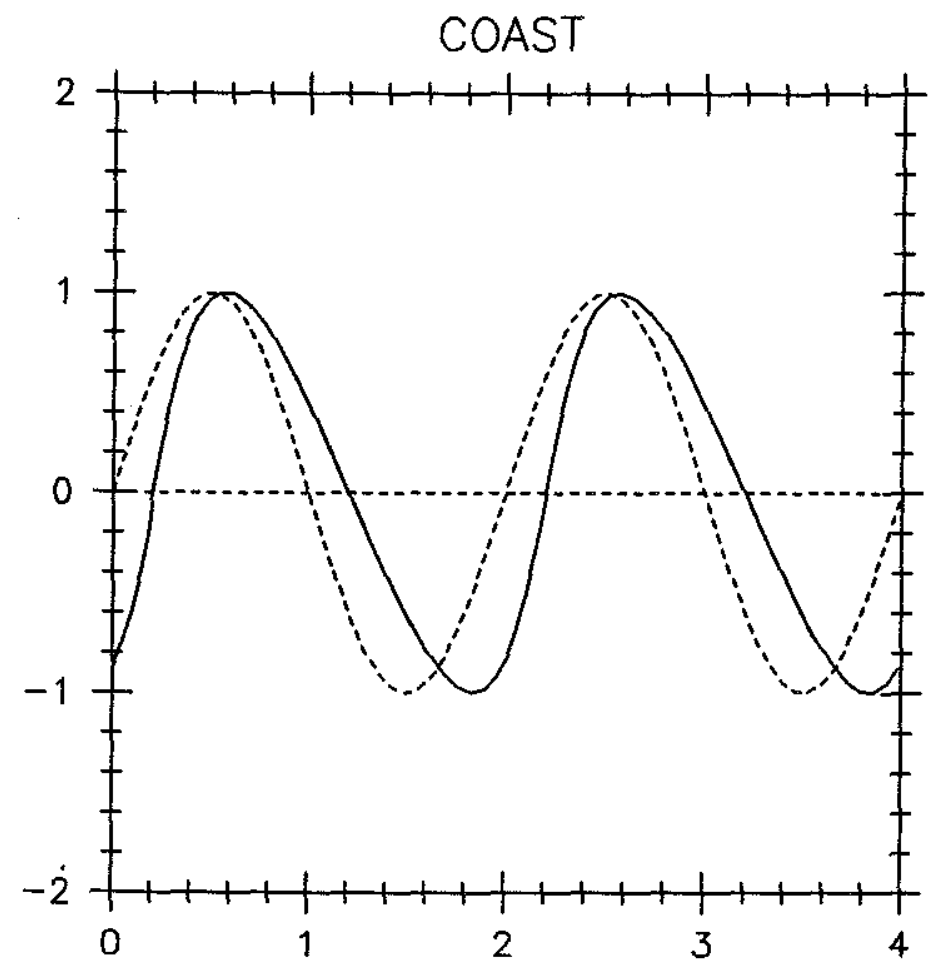

Figure 6.3: Modelled example of surface isotherm pattern due to wave steepening effect. The coastal barrier lies at the top of the figure. At a constant offshore distance seaward of the surface front (e.g. -1.2), the upper layer will be deep when the front is closest to the coast and shallow for the front farthest from the coast. 
isotherms appear spread apart compared to the inshore peaks. Evidence for this topographic effect is apparent in the widely spaced isotherms at the offshore peak near $\sim 45^{\circ} 15^{\prime} \mathrm{N}$ and the tightly packed isotherms at the inshore peak just to the south. While the above discussion may explain some of the observed compression/rarefaction of surface isotherms, the structure of the frontal zone is clearly more complicated. A stability model with better resolution of the frontal zone is needed to fully understand the observed surface temperature patterns.

The SST map from July 15 (Fig. 6.2c) exhibits a sharp surface front in the north $\left(45^{\circ} 10^{\prime} \mathrm{N}\right)$ connected with a weaker temperature gradient region to the south. The vertical section of density presented in Fig. 1.1 was taken across the sharp front in the north at $\sim 45^{\circ} 15^{\prime} \mathrm{N}$. There still exists alongfront variability in the position of the upwelling front, but the amplitude of the disturbance appears less than in the July 14 SST map. Furthermore, tracing the change in time of isotherm position (e.g. using the $49^{\circ}$ isotherm) shows that the entire meander pattern has propagated to the south. This propagation speed is quantified below. The sharp front in the northern part of the July 15 map is presumed to have come in to the study region from the north. This would be consistent if the wavelike meander pattern of July 14 repeated northward of the study area. Although measurements do not exist to prove this conjecture, the alternating pattern of closely packed isotherms at inshore peaks and widely spaced isotherms at offshore peaks would indicate, if the pattern did repeat northward, the existence of a region to the north of closely packed isotherms associated with the next inshore peak of the disturbance. This sharp frontal region would then presumably have propagated into the northern part of the SST map of July 15.

The next available SST map (July 16, not shown) exhibits a less organized pattern and contains several eddies of $\sim 30 \mathrm{~km}$ length in the alongshore direction. It is difficult to identify a surface front connected in the 
alongshore direction.

The above results provide a qualitative description of the growth of an alongfront meander on a coastal upwelling front off the coast of Oregon. In order to compare the observations to the stability model predictions, a quantitative estimate of the meander properties must be made. To establish an alongfront wavelength, individual SST maps are used to measure the alongfront distance between peaks of the wave. As noted above, the wavelength is different between the northern and southern parts of the disturbance. This difference may be due to an alongshore change in the shelf geometry or basic state flow, a feature which is absent in the simple stability model presented in the previous chapters. This subject will be discussed further in chapter 7. An estimate of the range of alongfront wavelengths may be obtained by measuring peak-to-peak distances from all three SST maps. Individual values obtained in this manner are contained in Table 6.1. The range of alongfront wavelengths is $32-52 \mathrm{~km}$.

While estimating alongshore phase propagation for the meander patterns is difficult, an attempt is made at least to establish the direction and get a rough estimate of the magnitude of the propagation speed. Two methods are used to determine the alongshore propagation speed. Changes in the alongshore position of the wave peaks between July 12 and July 14 and between July 14 and July 15 are averaged to obtain a propagation speed of $\sim 9 \mathrm{~cm} \mathrm{~s}^{-1}$ to the south. The individual estimates used in this average are contained in Table 6.1. The second method involves measuring the distance between the intersection of individual surface isotherms with the $50 \mathrm{fm}$ isobath from one SST map to the next. This method yields an average propagation speed of $\sim 8 \mathrm{~cm} \mathrm{~s}^{-1}$ toward the south, which is fairly close to the estimate from the first method.

An estimate of growth rate for this frontal wave can be found by examining the change in time of the cross-front peak-to-peak wave amplitude. This is most easily done between the July 12 and July 14 SST 
maps, with the result (individual values in the average are contained in Table 6.1) $\sigma_{i}^{-1} f^{-1} \sim 4$ days. This estimate of growth rate as well as those for alongfront wavelength and propagation speed should be treated as approximate, given the certain amount of subjectivity used in drawing the contour maps of SST. However, they provide reasonable estimates to compare to the properties predicted for the fastest growing wave in the stability model.

Before comparing observed frontal wave properties for this geographic location to those obtained from the stability model, a second time period will be examined. SST maps from July 22 and 23 are displayed in Fig. 6.4. The winds during this period (Fig. 6.1) vary daily between a $1 \mathrm{dyn} \mathrm{cm}^{-2}$ stress in an upwelling-favorable direction to little or no wind. A surface temperature front is evident in Fig. 6.4a separating cold, inshore water from the warmer water offshore. As during the previous time period (discussed above), a wavelike meander exists on the surface front. No SST information is available in the 3 days before the July 22 map, so the previous time history of the meander pattern is unknown. The wavelength of this feature is comparable to that of the July 12-15 meander, with the same tendency for a longer length scale in the northern part of the study region. The properties of this wave are quantified below. A continuously connected alongshore front is less obvious in the SST map from July 23 (Fig. 6.4b). In fact, the wavelike pattern of July 22 seems to have amplified and perhaps broken into closed or nearly closed eddies. Note the offshore eruption of the $56^{\circ}$ isotherm at the northern end of the study region, the deepening of the trough just to the south and the strengthening of the cold eddy at $\sim 44^{\circ} 50^{\prime} \mathrm{N}$. (Note that the above interpretation is only one possible explanation for the evolution of the surface temperature pattern and that processes such as mixed layer deepening could make following the motion of individual surface isotherms between maps problematic.) This behavior of the alongfront meander pattern is surely a finite amplitude phenomenon, 


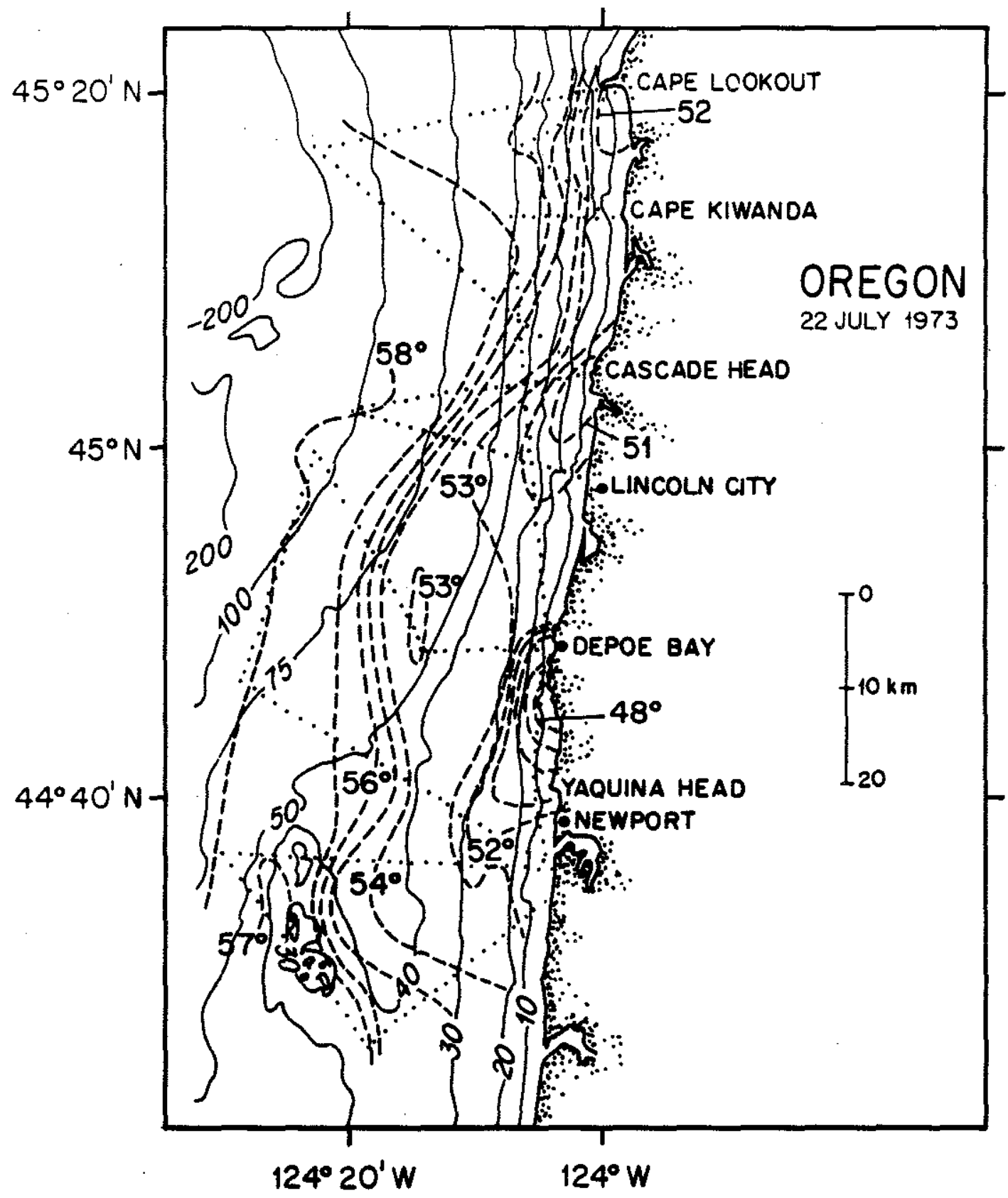

Figure 6.4: As in Fig. 6.2 but for (a) July 22 and (b) July 23. 


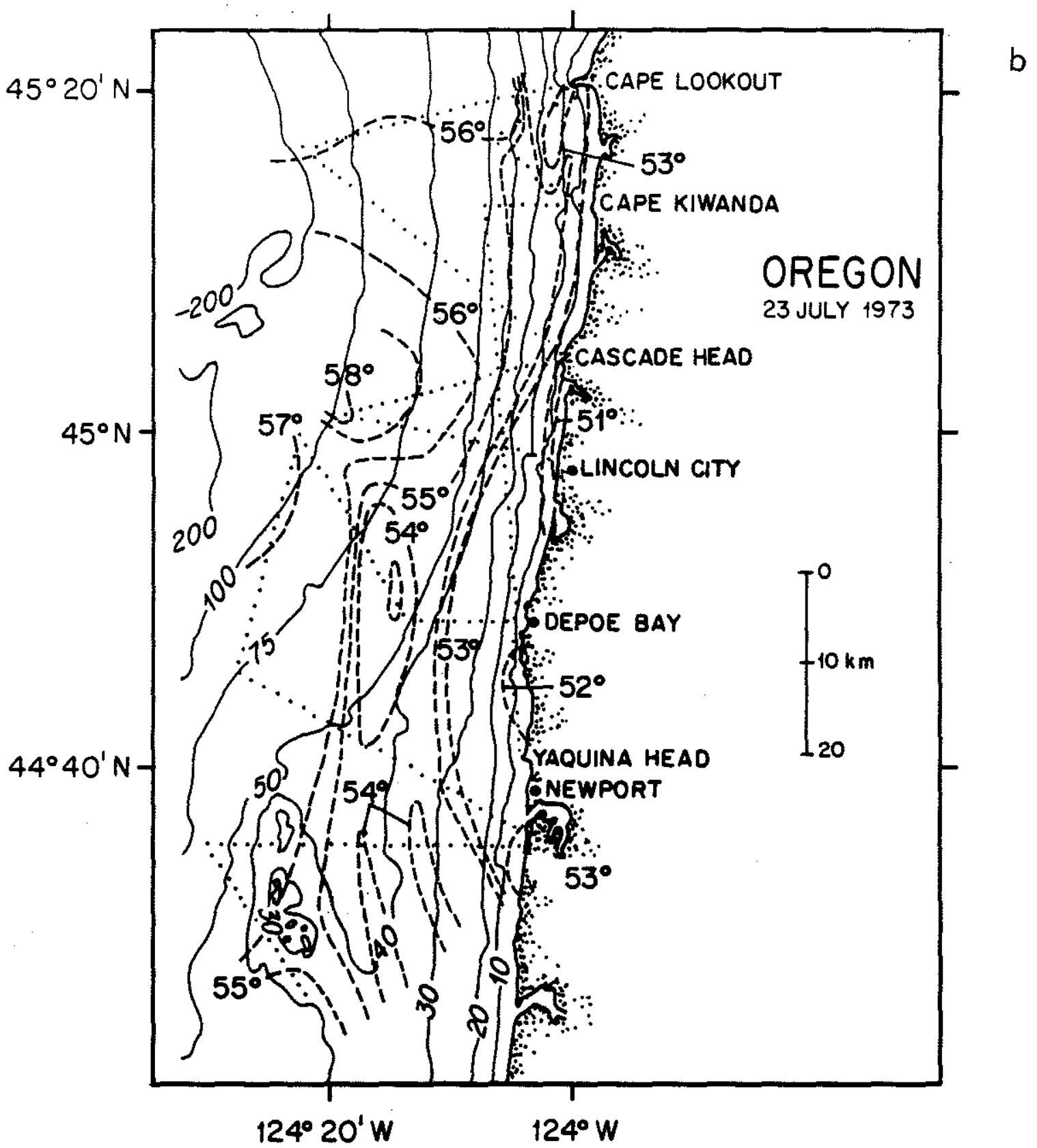


which suggests that the unstable waves predicted by the small amplitude model in the previous chapters can grow to finite amplitude. This sequence of SST images may offer an example of an alongfront meander beginning to disrupt the alongshore flow field by the formation of detached eddies.

The same techniques as used for the first time period are used to quantify the properties of this wavelike perturbation. The individual estimates used are contained in Table 6.2. The range of alongshore wavelengths is 31-60 km. An alongshore phase speed of $\sim 14 \mathrm{~cm} \mathrm{~s}^{-1}$ to the south is obtained from noting the change in time of the alongshore position of wave peaks. From examining the movement of individual isotherms along the $50 \mathrm{fm}$ isobath, a value of $\sim 11 \mathrm{~cm} \mathrm{~s}^{-1}$ is obtained. Growth rate is again a difficult property to estimate, but the e-folding time during this time period appears to be $\sim 2$ days, somewhat shorter than observed in the previous sequence of images.

The results from these two case studies of alongshore meanders in the coastal upwelling front off Oregon provide estimates of wave properties which can be compared to predictions from the simple two-layer stability model. The observed wave properties are

$$
\begin{aligned}
\text { alongfront wavelength } & \sim 31-60 \mathrm{~km}, \\
\text { phase speed } & \sim 8-14 \mathrm{~cm} \mathrm{~s}^{-1} \text { to the south, } \\
\text { e-folding time } & \sim 2-4 \text { days. }
\end{aligned}
$$

Since the properties of the fastest growing wave (i.e. the wave most likely to be observed as it grows rapidly from an initial disturbance field containing many wave components of varying alongfront scale) predicted by the stability model are greatly affected by the choice of layer depths and bottom topography, it is necessary to have an accurate estimate of these values for the study region off Oregon.

A constant upper layer depth far offshore of the surface front can be estimated from examining the vertical section of density displayed in Fig. 1.1. The value of $H_{1}$ is chosen to be $20 \mathrm{~m}$ with a density defect of $\delta=0.002$. 


\begin{tabular}{|c|c|c|c|}
\hline Date & $\begin{array}{c}\text { Wavelength } \\
\text { estimates }(\mathrm{km})\end{array}$ & $\begin{array}{c}\text { Phase speed } \\
\text { estimates }\left(\mathrm{cm} \mathrm{s}^{-1}\right)\end{array}$ & $\begin{array}{c}\text { cross-front } \\
\text { peak-to-peak } \\
\text { amplitude estimates }(\mathrm{km})\end{array}$ \\
\hline $7 / 12 / 73$ & 32,47 & & 6,7 \\
\hline & & $-9,11,15,12$ & \\
\hline $7 / 14 / 73$ & $47,52,34$ & & $9,10,14$ \\
\hline & & $-33,-36,-20,-12$ & \\
\hline $7 / 15 / 73$ & 39,34 & & \\
\hline \hline & average $=41 \mathrm{~km}$ & average $=-9 \mathrm{~cm} \mathrm{~s}^{-1}$ & \\
\hline
\end{tabular}

Table 6.1: Estimates of the properties of observed alongfront variability off the coast of Oregon.

\begin{tabular}{|c|c|c|c|}
\hline Date & $\begin{array}{c}\text { Wavelength } \\
\text { estimates }(\mathrm{km})\end{array}$ & $\begin{array}{c}\text { Phase speed } \\
\text { estimates }\left(\mathrm{cm} \mathrm{s}^{-1}\right)\end{array}$ & $\begin{array}{c}\text { cross-front } \\
\text { peak-to-peak } \\
\text { amplitude estimates }(\mathrm{km})\end{array}$ \\
\hline $7 / 22 / 73$ & 60,37 & & $7,8,4,6$ \\
\hline & & $-10,-21,+9,0$ & \\
\hline $7 / 23 / 73$ & $31,31,47$ & & 15,9 \\
\hline & & $-28,-33$ & \\
\hline $7 / 24 / 73$ & 47 & & \\
\hline \hline & average $=41 \mathrm{~km}$ & average $=-14 \mathrm{~cm} \mathrm{~s}^{-1}$ & \\
\hline
\end{tabular}

Table 6.2: Estimates of the properties of observed alongfront variability off the coast of Oregon. 
With these values, the internal Rossby radius of deformation $(R)$ is $6.2 \mathrm{~km}$ and the long internal gravity wave phase speed $\left(\left(g^{\prime} H_{1}\right)^{1 / 2}\right)$ is $63 \mathrm{~cm} \mathrm{~s}^{-1}$. From Fig. 1.1 it is difficult to determine the interface profile due to the presence of many small scale features. In the stability model, the interface is modelled as an exponential with an adjustable e-folding scale. It was decided to use $\alpha=1.0$ as a reasonable first guess, rather than estimate an appropriate e-folding scale for the interface from observations. As described in section 5.3, increasing $\alpha$ will make the interface rise more steeply to the surface, increase the maximum velocity at the surface front and, as a result, increase the growth rate of the fastest growing wave. However, the wavenumber of the fastest growing mode will not be greatly affected by a change in $\alpha$ (see Fig. 5.10). With $\alpha=1.0$, the maximum upper layer mean flow velocity is $63 \mathrm{~cm} \mathrm{~s}^{-1}$ to the south at the surface front. This value is consistent with the geostrophic velocity estimates of Curtin (1979) who found velocities of $\sim 40-50 \mathrm{~cm} \mathrm{~s}^{-1}$ at $20 \mathrm{~m}$ depth near the surface front. For the above reasons, the model interface parameter is chosen as $\alpha=1.0$.

The cross-shelf bottom topography varies alongshore in the study region (Fig. 6.2), an effect which is not included in the simple stability model. This omission is discussed further in chapter 7. The cross-shelf bottom topography used in the stability model is displayed in Fig. 6.5. Other cross-shelf sections in the region will have more steeply or more gently sloping continental shelves, but the topography displayed in Fig. 6.5 is fairly representative. As discussed in section 5.4, an increase in the bottom slope will decrease the growth rate and the alongfront wavenumber of the fastest growing wave and a decrease in the bottom slope will have the opposite effect. Again, in an effort to use the simple stability model to get an estimate for the unstable wave properties, the topography displayed in Fig. 6.5 is deemed a realistic choice. Also shown in Fig. 6.5 is the scaled bottom slope parameter $s$ as a function of cross-shelf distance. This value is obtained by multiplying the actual physical bottom slope by $R / H_{1}$. As 


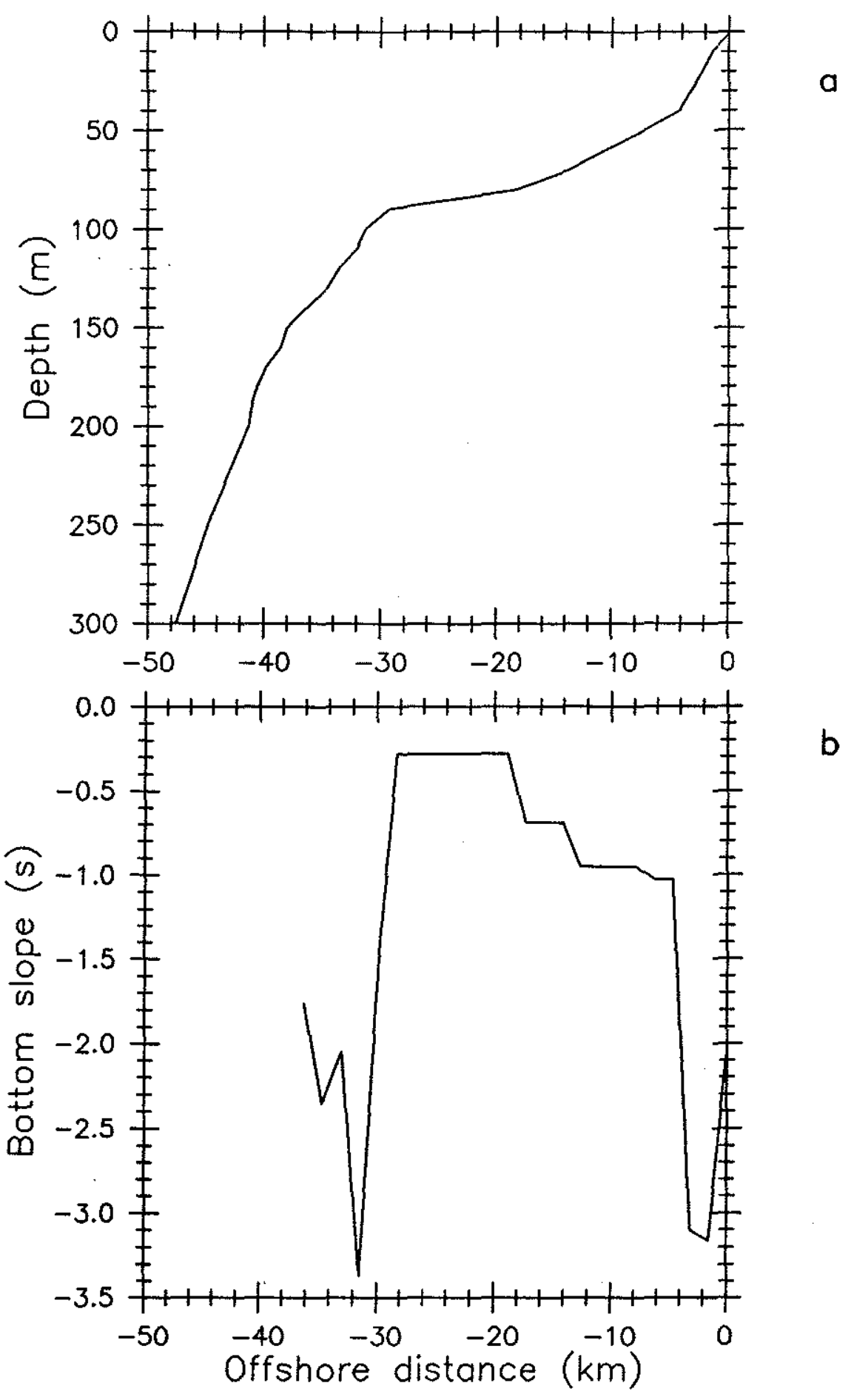

Figure 6.5: (a) Bottom topography off the coast of Oregon used in the stability model. (b) Bottom slope parameter $s$ for the cross-shelf topography in (a). The parameter $s$ is related to the actual physical bottom slope $s_{*}$ by $s=\left(R / H_{1}\right) s_{*}$. 
noted in section 5.4, with $\alpha=1.0$ a value of $s \leq-1.0$ beneath the surface front will stabilize the front. The bottom topography in Fig. 6.5 contains a range of the values of $s$, so it may be anticipated that the offshore location of the surface front will determine its stability properties. Another point to keep in mind is that deeper lower layers decrease the growth rate and alongfront wavenumber of the fastest growing wave (Fig. 5.9). Therefore, it may be anticipated that the fastest growing mode will occur when the surface front lies over a weakly sloping bottom in shallow water.

Partial growth rate curves for three values of the distance between the surface front and the coast $\left(x_{f}\right)$ are displayed in Fig. 6.6. The dimensional offshore position of the surface front is obtained by multiplying the nondimensional value $x_{f}$ by $R=6.2 \mathrm{~km}$. In addition, no unstable waves are found for a model with $x_{f}=-2.0 R(-12.4 \mathrm{~km})$. The largest value of the fastest growing wave's growth rate occurs for $x_{f}=-3.0 R(-18.6 \mathrm{~km})$. This mode has an alongfront wavenumber of $l=0.75$. The maximum growth rates for $x_{f}=-2.5 R(-15.5 \mathrm{~km})$ and $x_{f}=-3.5 R(-21.7 \mathrm{~km})$ are less, but occur at a wavenumber only slightly less than that found for $x_{f}=-3.0 R$. The dimensional properties of these fastest growing modes are obtained by multiplying $l^{-1}$ by $2 \pi R$ to get alongfront wavelength, $\sigma_{i}^{-1}$ by $f^{-1}$ to get e-folding time and $c_{r}$ by $\left(g^{\prime} H_{1}\right)^{1 / 2}$ to get alongfront phase propagation. The results for this range of $x_{f}$ are:

$$
\begin{array}{cl}
\text { alongfront wavelength } & 52-56 \mathrm{~km}, \\
\text { phase speed } & 1-2 \mathrm{~cm} \mathrm{~s}^{-1} \text { to the south, } \\
\text { e-folding time } & 5-8 \text { days. }
\end{array}
$$

The alongfront wavelengths predicted by the two-layer stability model are within the range of the observed values. However, the model underpredicts both the phase speed toward the south and the growth rate. These properties may be modified by adjusting the model input parameters. For example, increasing $\alpha$ (i.e. making the model front rise more sharply to the surface) will increase the phase speed toward $-y$ and decrease the e- 


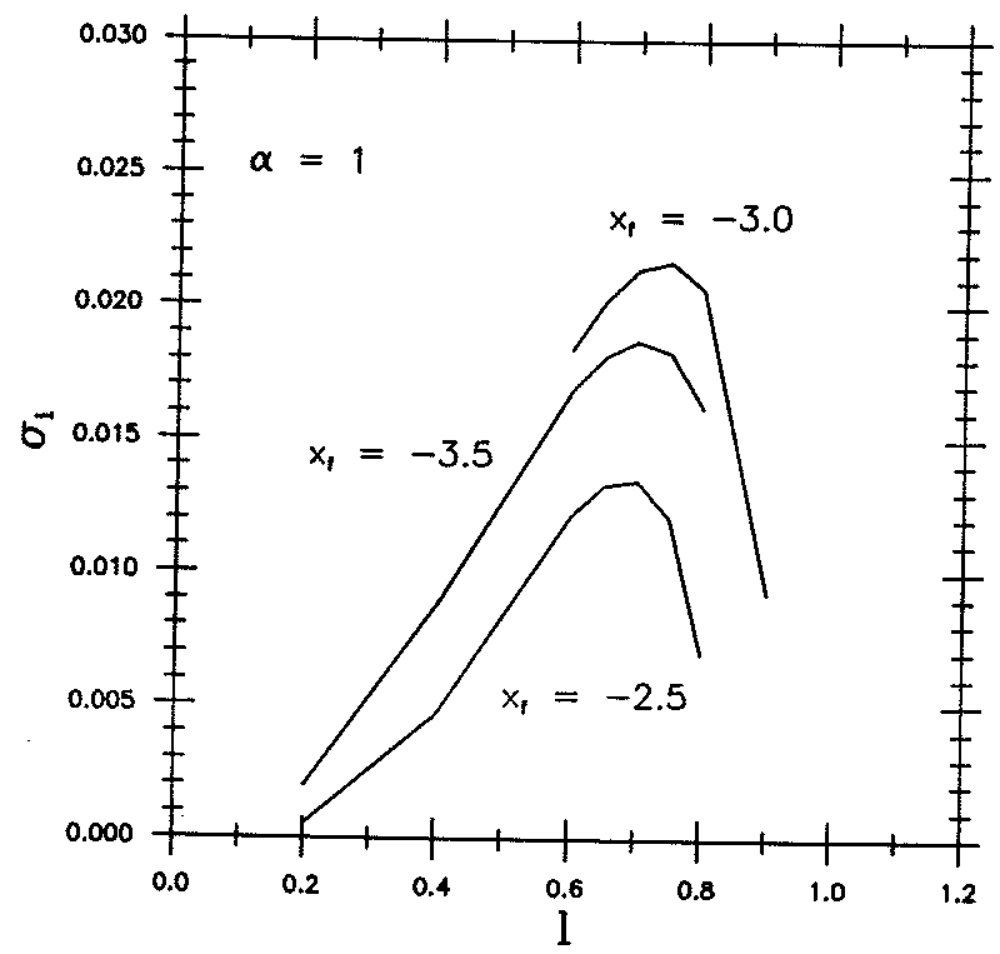

Figure 6.6: Partial growth rate $\left(\sigma_{i}\right)$ curves for the modelled upwelling front off the coast of Oregon. Values on the abscissa are alongfront wavenumbers (l). 
folding time due to the increased vertical shear. The vertical section of density in Fig. 1.1 provides an indication that the pycnocline may rise to the surface sharply. Another possible explanation for the underprediction of phase speed and growth rate is the lack of stratification beneath the model front. Underlying stratification may isolate the upper water column form the influence of the sloping bottom. This point is addressed further in chapter 7 below. However, given the best guess input parameters used here, the simple two-layer stability model predicts properties of the fastest growing wave which are fairly close to the observed values. Therefore, the existence of growing alongfront meanders on coastal upwelling fronts in this region may be interpreted in terms of an internal instability process.

One final note concerns the effect of linearized bottom friction on the unstable wave. As discussed in section 5.5, when the frictional damping time scale is long compared to the e-folding time scale of the most unstable wave, the effect of friction will be to decrease the growth rate of the fastest growing mode, increase its phase speed toward $-y$ and shift its wavelength only slightly (see Fig. 5.16). An estimate of the frictional time scale for the model front off Oregon using $\lambda_{\psi}=0.04 \mathrm{~cm} \mathrm{~s}^{-1}$ is 18 days. Since this time scale is long compared to the growth rates predicted by the inviscid model (5-8 days), bottom friction is expected to decrease the fastest growing wave's growth rate and increase its phase speed to $-y$. In fact, running the viscous stability model with a bottom friction parameter of $\lambda_{*}=0.04 \mathrm{~cm} \mathrm{~s}^{-1}$ results in the following wave properties

$$
\begin{array}{cl}
\text { alongfront wavelength } & 37-43 \mathrm{~km}, \\
\text { phase speed } & 3-6 \mathrm{~cm} \mathrm{~s}^{-1} \text { to the south, } \\
\text { e-folding time } & 7-11 \text { days. }
\end{array}
$$

In summary, bottom friction shifts the predicted phase speed closer to the observed values, but contributes further to the model underprediction of growth rate. 


\subsection{Nova Scotia}

Recently, Petrie et al. (1987) presented observational evidence for unstable waves on a coastal upwelling front which formed off the east coast of Nova Scotia. During the month of July 1984, a weak but persistent alongshore wind blew in an upwelling-favorable direction (Fig. 6.7). In response, cold saline water was upwelled at the coast forming an offshore surface front. An alongfront meandering with wavelike characteristics was observed to grow on this surface front. A satellite SST image from July 25 near the end of the period of upwelling-favorable winds is presented in Fig. 6.8a. A chart with a latitude-longitude grid, place names and the offshore location of the shelf break (as indicated by the $200 \mathrm{~m}$ isobath) is included in Fig. $6.8 \mathrm{~b}$. The waters along the coast which appear white have a temperature of 10-14 $4^{\circ} \mathrm{C}$. The gray areas inshore of this band have temperatures as low as $4^{\circ} \mathrm{C}$. The dark gray waters offshore of the white upwelled band are warmer than $14^{\circ} \mathrm{C}$. The light area to the southeast has temperatures of 18-22 $2^{\circ} \mathrm{C}$. A Gulf Stream ring with temperatures $\sim 26^{\circ} \mathrm{C}$ is represented by the nearly black, circular region in the center at the bottom of the figure. Three large meanders in the coastal upwelling front extend offshore between Halifax and the southwest tip of Nova Scotia. There is also evidence for two more, smaller amplitude, meanders along the front to the northeast. These perturbations are fairly evenly spaced alongshore and have a wavelength in the range of $50-75 \mathrm{~km}$ (Petrie et al., 1987).

The growth of these meanders can be seen in a time sequence of satellite SST images presented in Fig. 6.9. The earliest image taken on July 7 (Fig. 6.9a) shows very little cold upwelled water near the coast. The image from a week later (July 14, Fig. 6.9b) shows a much larger region of cold water along the Nova Scotian coast and around into the Bay of Fundy. The alongfront perturbations in the surface front have begun to form at this time. The three southernmost wavelike meanders grow to rather large 


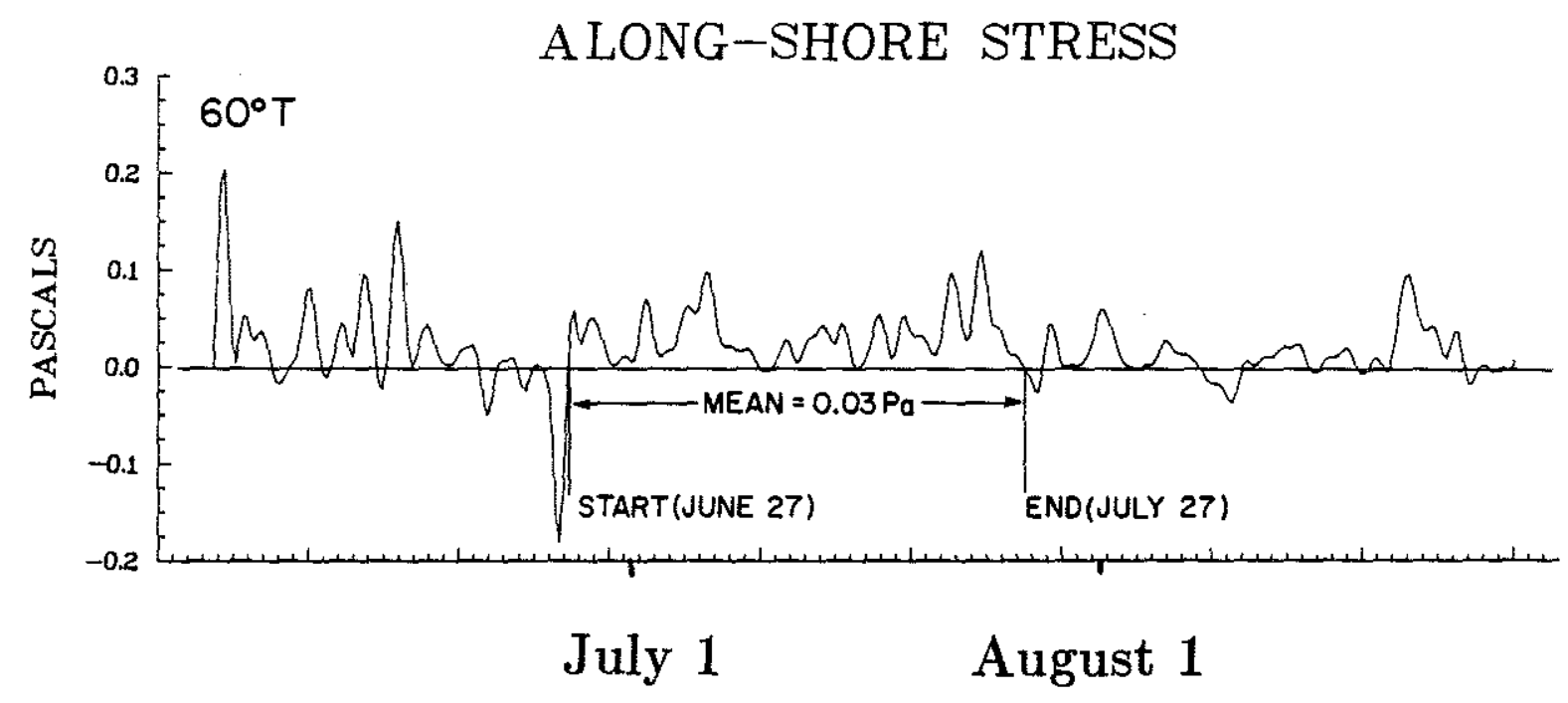

SABLE ISLAND WIND STRESS

Figure 6.7: Alongshore wind stress $\left(60^{\circ} \mathrm{T}\right)$ observed at Sable Island (see Fig. 6.8b) $\left(0.1 \mathrm{~Pa}=1.0 \mathrm{dyn}^{-2}\right)$. From Petrie et al., 1987. 


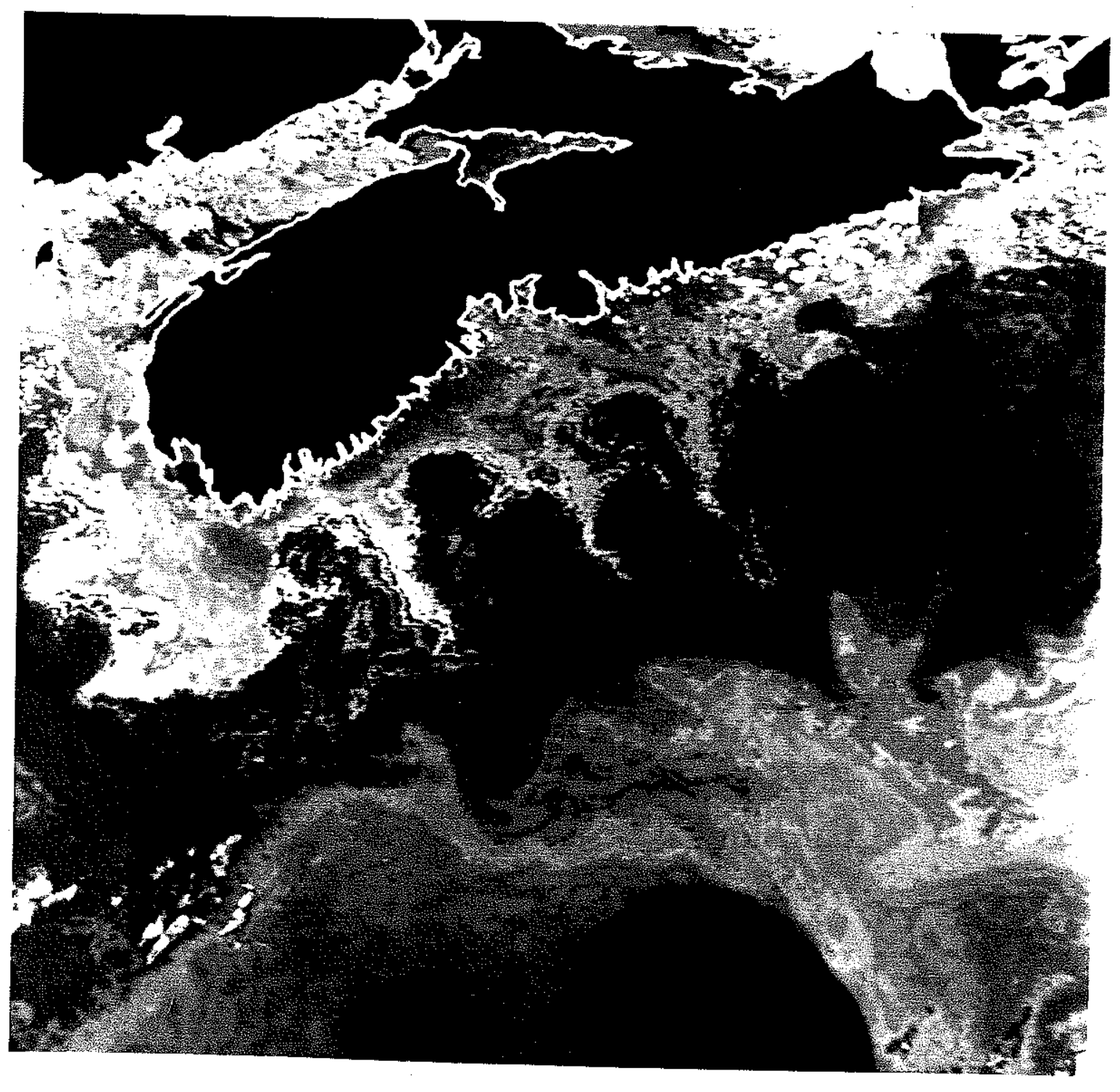

a
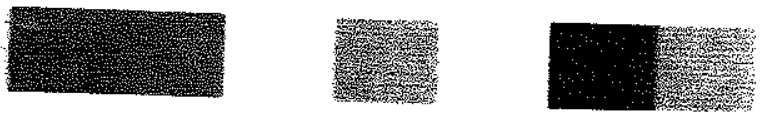

0

2

4

68

$10 \quad 12$

14

16

18

$\begin{array}{lll}20 & 22 & 24\end{array}$

26

Figure 6.8: (a) Satellite (NOAA-7) sea surface temperature image for July 25, 1984. (b) Location map for Nova Scotia case study. From Petrie et al., 1987. 


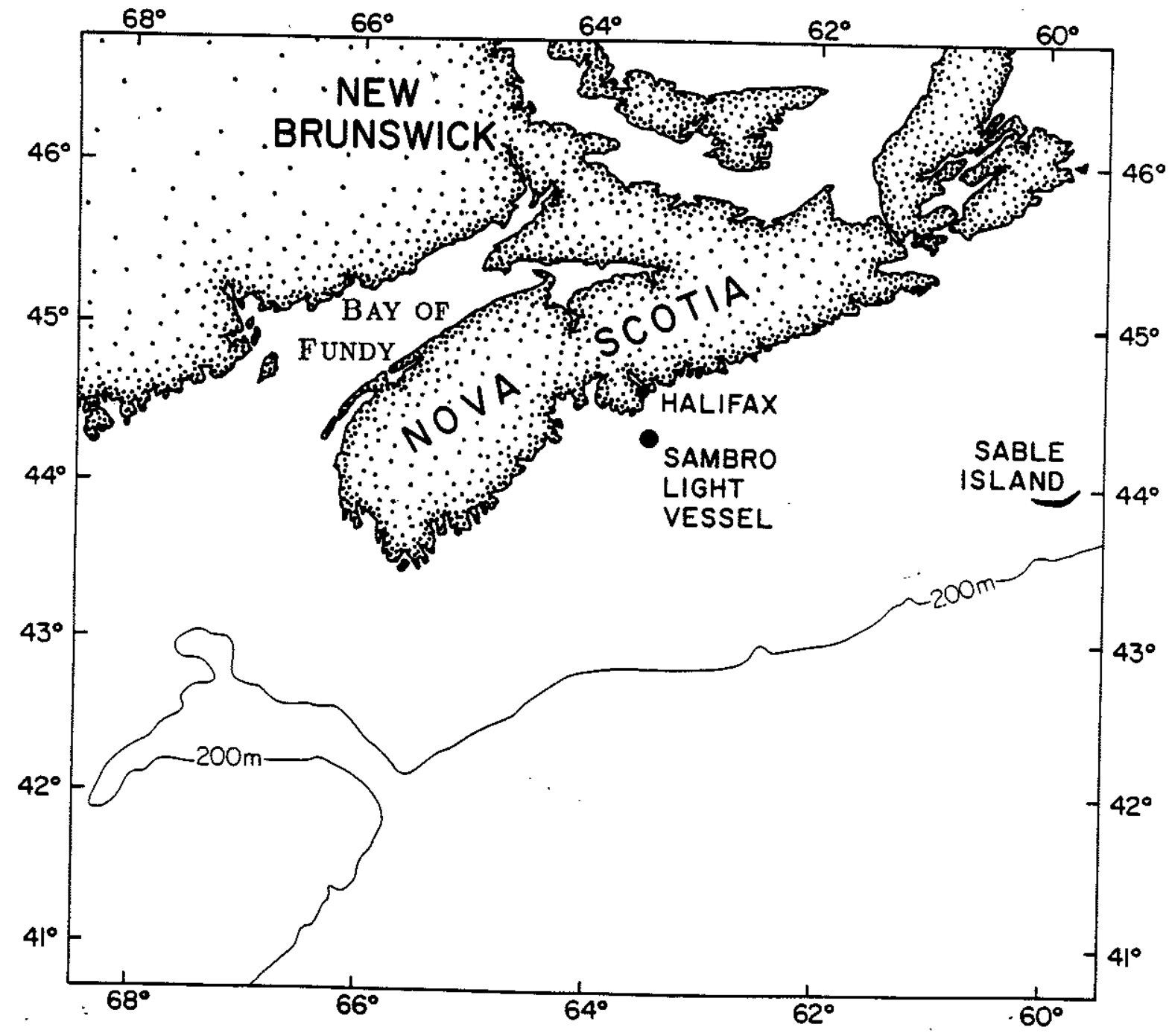



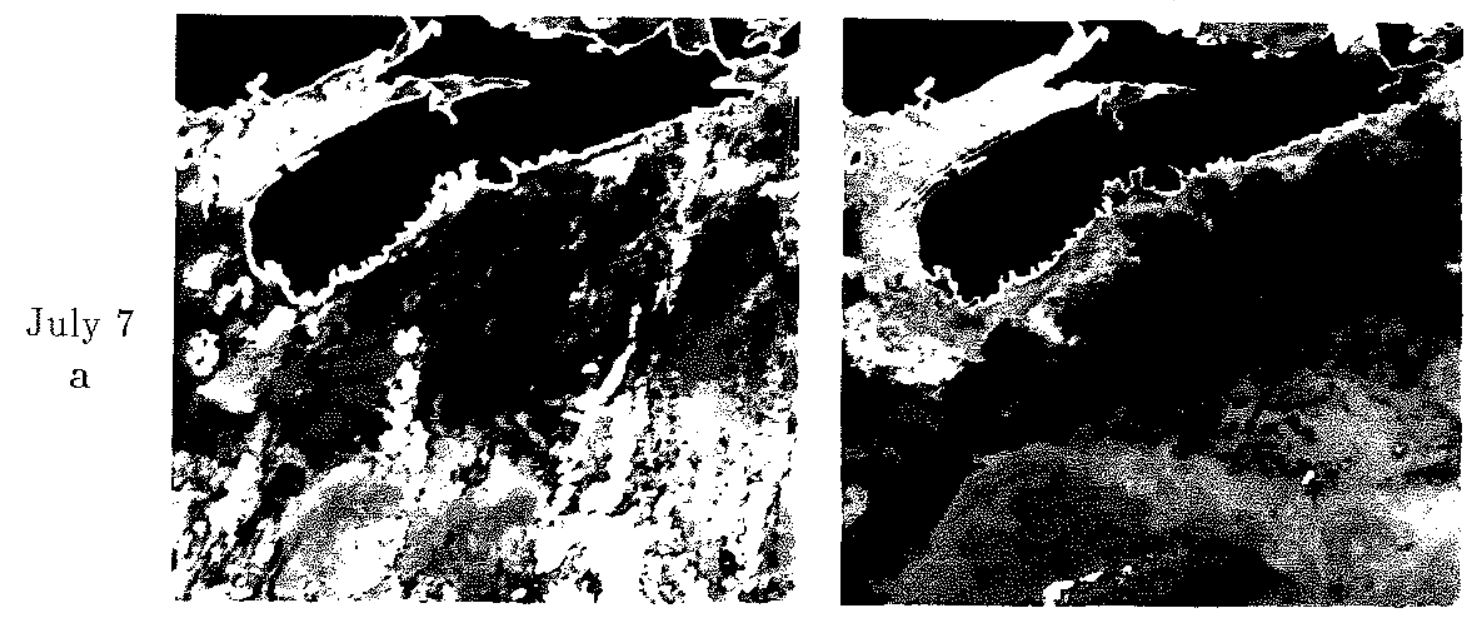

$\underset{\mathrm{d}}{\mathrm{July}} 14$
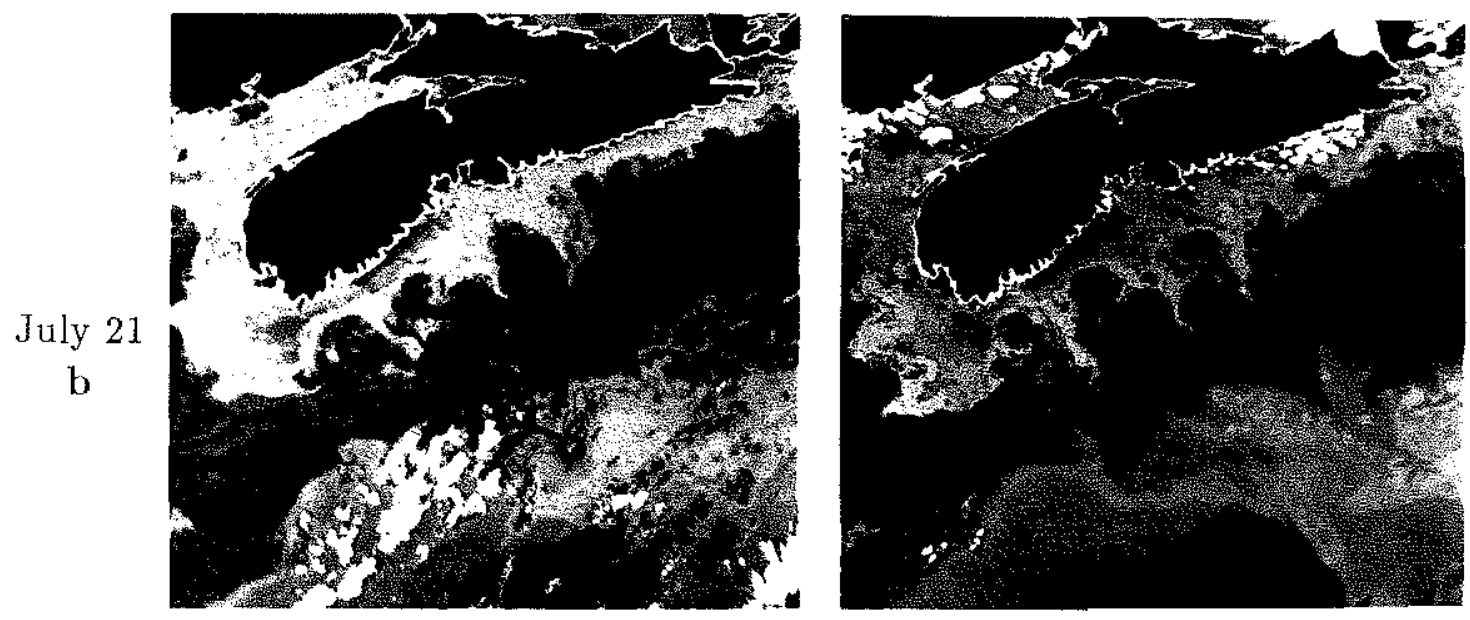

July 25

e
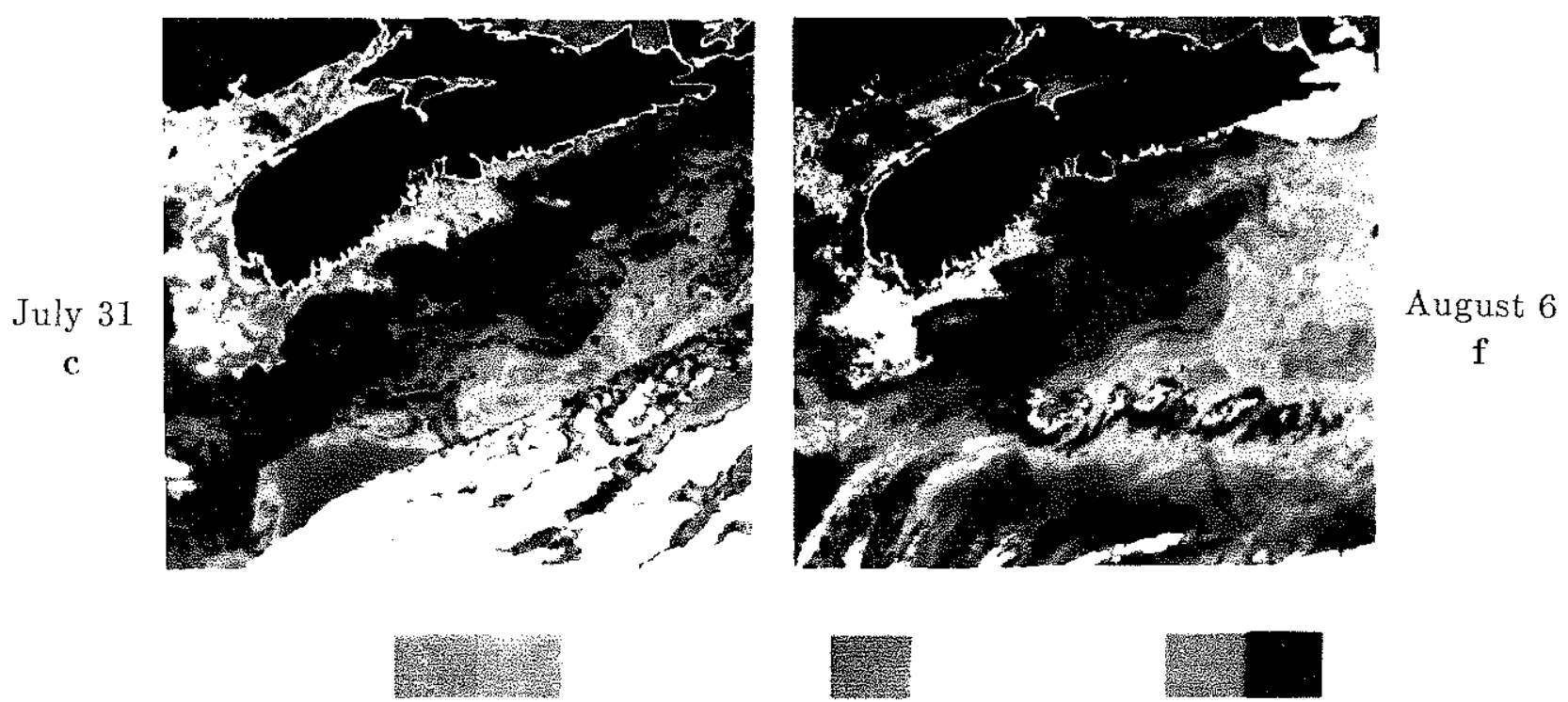
$0 \quad 2$
6
$\begin{array}{lll}8 & 10 & 12\end{array}$
$\begin{array}{lll}14 & 16 & 18\end{array}$
$\begin{array}{llll}20 & 22 & 24 & 26\end{array}$

Figure 6.9: Series of satellite SST images: (a) July 7; (b) July 14; (c) July 21; (d) July 25; (e) July 31; and, (f) August 6. From Petric et al., 1987. 
amplitude by the July 21 SST image (Fig. 6.9c). The image in Fig. 6.9d is the same as that shown in Fig. 6.8a, and shows the fully developed meanders extending up to $\sim 75 \mathrm{~km}$ away from the initial surface front. In the following weeks (July 31, Fig. 6.9e and August 6, Fig. 6.9f) the area of upwelled water decreases in size and warms. At the same time the alongfront meanders decrease in size.

From this series of satellite SST images the existence of growing frontal perturbations is clearly established. Note the very small propagation speed of the meander pattern. By superimposing successive images, Petrie et al. (1987) find a phase propagation to the northeast in the range of 0$2.0 \mathrm{~cm} \mathrm{~s}^{-1}$. These features grow quite rapidly as is evident in Fig. 6.9. Petrie et al. (1987), using additional SST images not presented here, estimate the e-folding time to be of order several days. In summary, the observed unstable wave properties are

$$
\begin{array}{cl}
\text { alongfront wavelength } & 50-75 \mathrm{~km}, \\
\text { phase speed } & 0-2 \mathrm{~cm} \mathrm{~s}^{-1} \text { to the northeast, } \\
\text { e-folding time } & \text { several days. }
\end{array}
$$

As was done for the case study off the coast of Oregon, estimates of layer depths and bottom topography need to be made in order to predict wave properties from the two-layer stability model. No detailed subsurface density sections across the upwelling front are available from this time period, but two hydrographic stations were occupied on July 29 and 31 (Fig. 6.10). One station is located just off the coast near Halifax within the band of cold water and the other is located $\sim 75 \mathrm{~km}$ offshore, outside the cold band. The inshore station has lower temperature and higher salinity (hence greater density) in the upper $15 \mathrm{~m}$ of the water column. As Petrie et al. (1987) note, these characteristics are consistent with coastal upwelling. The offshore station allows an estimate of $20 \mathrm{~m}$ for $H_{1}$ with a density defect of $\delta=0.003$. This gives a long internal gravity wave speed of $76 \mathrm{~cm} \mathrm{~s}^{-1}$ and an internal Rossby radius of deformation equal to $7: 4 \mathrm{~km}$. 

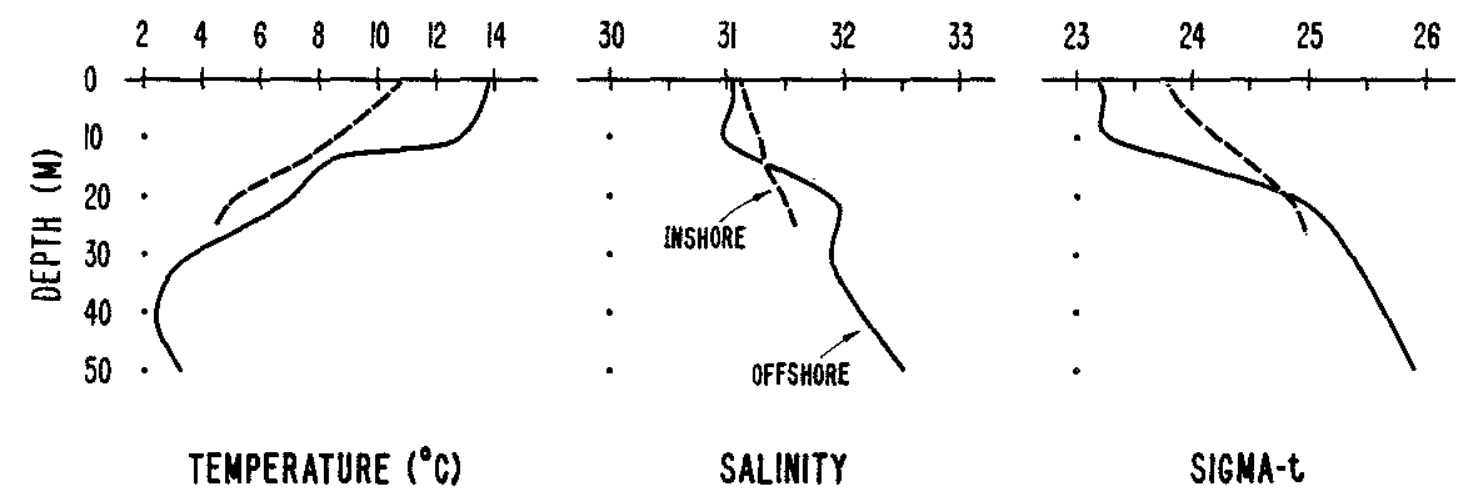

SALINITY

SIGMA-t

Figure 6.10: Temperature, salinity and density profiles for (a) nearshore (July 31, dashed curves) and (b) offshore (July 29, solid curves) stations off Halifax. From Petrie et al., 1987. 
The bottom topography off the east coast of Nova Scotia is rather complicated. The water depth increases rapidly nearshore reaching a value of $100 \mathrm{~m}$ within $10-12 \mathrm{~km}$ of the coast. Offshore of this the bottom slopes gently with a nearly constant linear value of $\sim 1 \times 10^{-3}$ (Petrie et al., 1987) to a deep trough $\sim 80 \mathrm{~km}$ offshore. Finally, an offshore bank gives way to a steep continental slope beginning at $\sim 180 \mathrm{~km}$ offshore. Since the satellite SST images indicate that the surface front lies over the region of approximately constant bottom slope, the two-layer stability model was run with a linearly sloping bottom next to a vertical coastal barrier as in section 5.4. The final necessary model input is the offshore distance to the surface front which is estimated from the satellite SST image to be $\sim 2$ $R(\sim 15 \mathrm{~km})$. This places the surface front in water of $110 \mathrm{~m}$ depth or $5.5 \mathrm{H}_{1}$. Since no details of the cross-front subsurface density structure are available, the interface parameter $\alpha$ is chosen equal to one.

With these choices the stability model predicts the following properties for the fastest growing wave

$$
\begin{array}{cl}
\text { alongfront wavelength } & 74 \mathrm{~km}, \\
\text { phase speed } & 1.0 \mathrm{~cm} \mathrm{~s}^{-1} \text { to the northeast, } \\
\text { e-folding time } & 10 \text { days. }
\end{array}
$$

The predicted wavelength and phase speed are within the range of observed variability. However, as was the case off Oregon, the model underpredicts the growth rate of the fastest growing mode. Again, the model input parameters may be varied. For example, a $20 \%$ increase in the vertical shear (obtained by increasing $\alpha$ to 1.2 ) results in no change in alongfront wavenumber, but increases the alongfront phase propagation to $2 \mathrm{~cm} \mathrm{~s}^{-1}$ to the northeast and decreases the e-folding time to 7 days. Another possibility, as mentioned in conjunction with the example from Oregon, is that underlying stratification may isolate the upwelling front from the stabilizing effect of the sloping bottom.

Another consideration is the reversed bottom slope between the deep 
trough $\sim 80 \mathrm{~km}$ offshore and the bank which reaches its shallowest point $\sim$ $160 \mathrm{~km}$ offshore. Even though the satellite images show that the upwelling front is never found that far offshore, it is of interest to analyze the effect of the reversed bottom slope on the properties of the fastest growing wave. Running the model with the surface front $100 \mathrm{~km}$ from the coast where the water depth is $\sim 200 \mathrm{~m}$ and the bottom slope is reversed, results in an efolding time for the fastest growing wave which is approximately half of the value state above. This shows the strong influence of bottom topography on the stability of the two-layer model. Again, the presence of underlying stratification may isolate the upwelling front from the destabilizing effect of a reversed bottom slope.

Bottom friction may also be included in this model. Since the water depth is large beneath the surface front, the frictional time scale is expected to be long. Using a value of $\lambda_{*}=0.04 \mathrm{~cm} \mathrm{~s}^{-1}$ (most likely an overestimate for this shelf region), this scale will be $\sim 30$ days. Clearly, the fastest growing wave in the inviscid model grows on a time scale shorter than this. Running the viscous stability model with $\lambda_{*}=0.04 \mathrm{~cm} \mathrm{~s}^{-1}$ results in little change in the unstable wave's growth rate, but shifts its wavelength to $\sim 60 \mathrm{~km}$ and its alongfront phase speed to $\sim 4 \mathrm{~cm} \mathrm{~s}^{-1}$ to the northeast.

Petrie et al. (1987) use a three-layer quasi-geostrophic model to study this phenomenon since they find that a two-layer quasi-geostrophic model without horizontal shear is completely stabilized by the strong bottom slope. The presence of a lower layer with zero mean flow effectively isolates the two upper flowing layers from the stabilizing influence of a sloping bottom. Even though the large interface displacements associated with coastal upwelling fronts make the quasi-geostrophic models formally invalid, they obtain unstable waves with the properties of the fastest growing wave in the range of observed values. Their predicted alongfront phase speed of 4-5 $\mathrm{cm} \mathrm{s}^{-1}$ to the northeast is larger than that expected from the observations. They suggest that adding a $3 \mathrm{~cm} \mathrm{~s}^{-1}$ depth-independent flow 
to the southwest in the system will bring this predicted value in line with observations. The authors also apply a constant depth two-layer shallow water equation model (Killworth et al., 1984 and section 5.3 in this study) to the problem. Again, predicted wave properties are within the range of observed values except for an excessive northeastward phase propagation.

The results presented here are from a model which is more physically realistic than either of the above two models. Large interface displacements and strong bottom slopes are allowed. Two points are noteworthy. The first is that sloping bottom topography representative of the continental shelf off the east coast of Nova Scotia does not stabilize the two-layer frontal model as it did the two-layer quasi-geostrophic model with uniform mean flow in each layer. Second, as discussed in section 5.3, a sloping bottom induces a shift in the phase speed of the unstable waves. The sense of this shift is to add a component in the direction that topographic vorticity waves propagate (with shallow water on the right in the northern hemisphere). Since the modelled unstable frontal waves propagate in the downstream direction (toward the northeast off the east coast of Nova Scotia during the period of active upwelling described here), a sloping bottom will decrease the magnitude of the phase speed. The small values of alongfront phase speed to the northeast observed for unstable waves in this area and predicted by the stability model is likely due to this effect.

\subsection{Southwestern Africa}

The coastal ocean off the southwest tip of Africa is an area of active upwelling (Bang, 1973). During the austral summer, winds from the southeast drive an upwelling circulation which creates coastal upwelling fronts. Upwelling has been observed from south of Cape Point $\left(34^{\circ} \mathrm{S}\right)$ to well north of Hondeklip Bay $\left(30^{\circ} \mathrm{S}\right)$ (Fig. 6.11). A number of regions of locally intense upwelling exist within this area (Taunton-Clark, 1985). Though these 

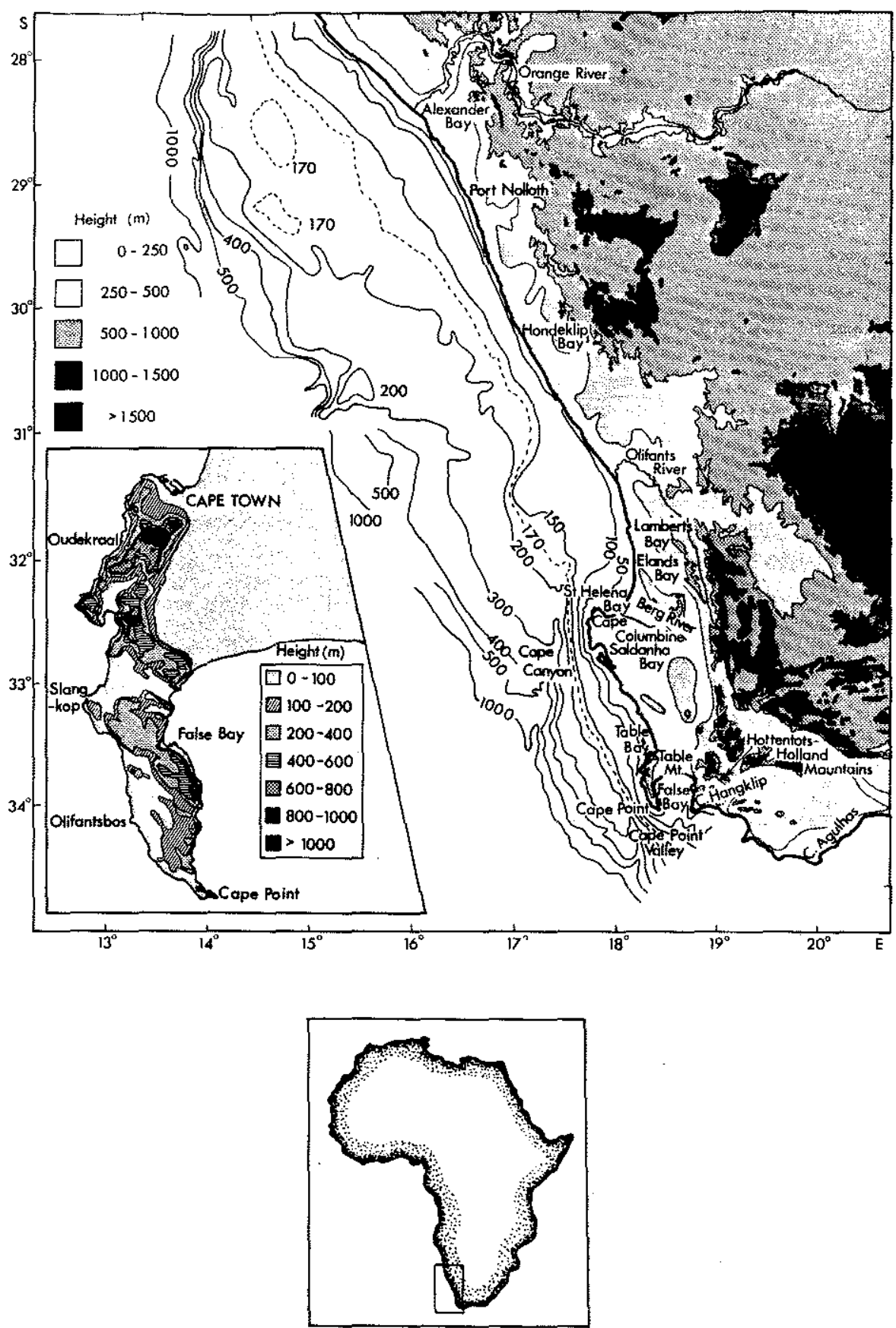

Figure 6.11: Location map for southwest Africa case study. 
upwelling structures are three-dimensional, there are areas where a fairly two-dimensional front forms alongshore. One such area is west of Cape Town between Cape Point and Cape Columbine. A number of observational studies have taken place in this region including the collection of a large set of aircraft derived SST maps (Taunton-Clark, 1982). The purpose of this section will be to describe the growth of an alongfront meander on the coastal upwelling front as portrayed in a series of daily SST maps. Estimates for the scales of the disturbance and a comparison to the results of the simple stability model will be made.

During January 17-28, 1980 the wind consistently blew in an upwelling favorable direction (Jury, 1984). Within this period, the wind stress is fairly constant at $\sim 1 \mathrm{dyn} \mathrm{cm}^{-2}$ from January 21-27. On January 22 an aircraft SST survey was done from south of Cape Point to north of Cape Columbine (Fig. 6.12a). The alongshore spacing of the sampling grid (not shown) is $\sim 25 \mathrm{~km}$. Cold water is found adjacent to the coast with an upwelling tongue extending northward from the Cape Peninsula $\left(34^{\circ} \mathrm{S}\right)$. Offshore of this tongue is a region of compressed surface isotherms running alongshore over most of the survey region. This coastal upwelling front, centered on $\sim 50 \mathrm{~km}$ offshore, is the region of interest here. On January 22 there appears to be an alongfront meander with two peaks away from the coast at $\sim 33^{\circ} 15^{\prime} \mathrm{S}$ and $\sim 34^{\circ} 15^{\prime} \mathrm{S}$. The distance between these two peaks is $\sim 125 \mathrm{~km}$. On January 23 (Fig. 6.12b), most of the SST features are still present from the previous day. The offshore surface front seems to have sharpened in the southern half of the study region. The alongfront meander is still present and appears to have shifted slightly northward (e.g. see the $15^{\circ}$ isotherm). At the southern end of the survey region another peak in the alongfront meander is now evident.

The SST map from January 24 (Fig. 6.12c) shows the amplification of the alongfront meander. All three peaks away from the coast increase in amplitude whereas the inshore peaks do not. This is most likely because 

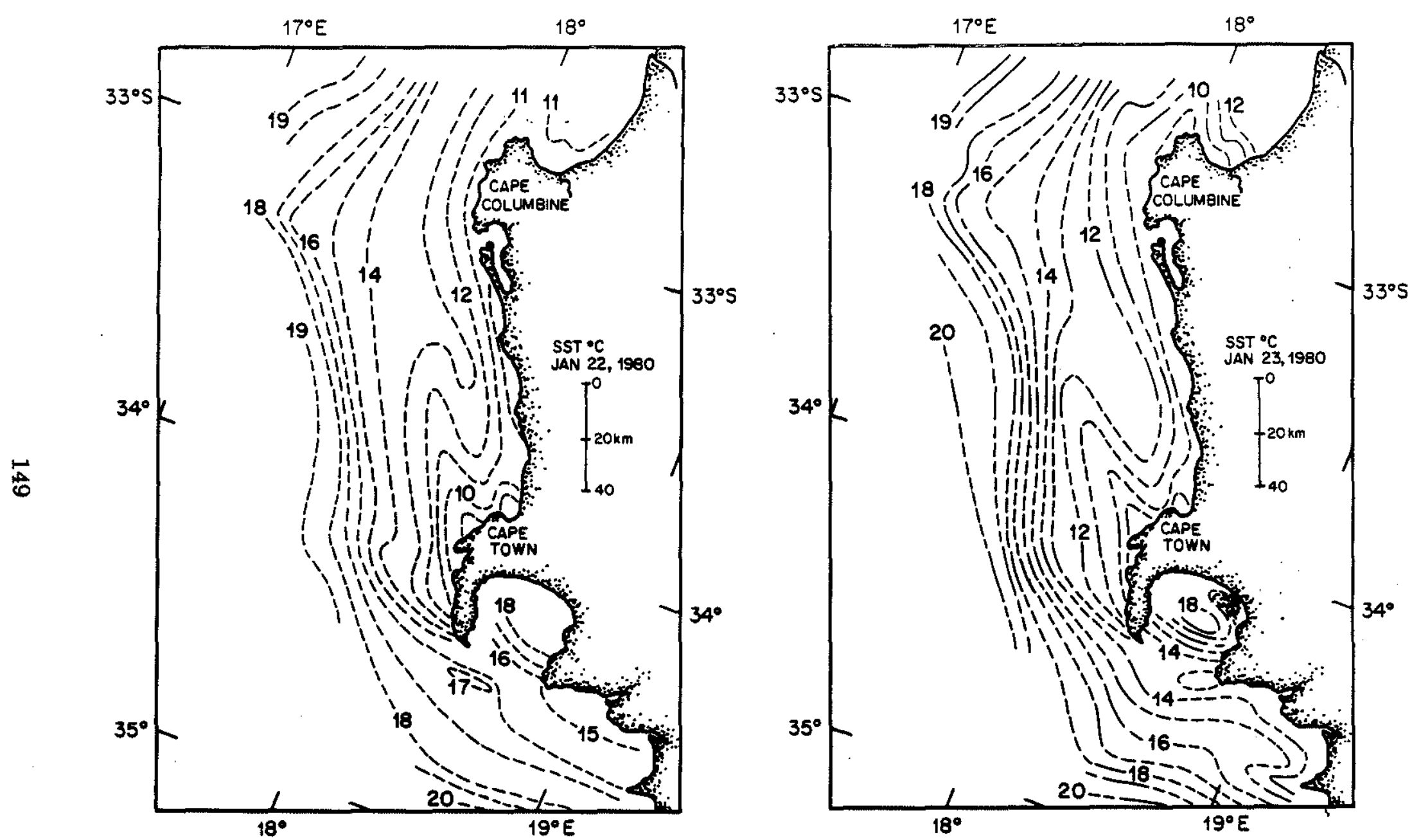

Figure 6.12: Aircraft SST maps for (a) Jan. 22, (b) Jan. 23, (c) Jan. 24 and (d) Jan. 25. From Taunton-Clark, 1982. 

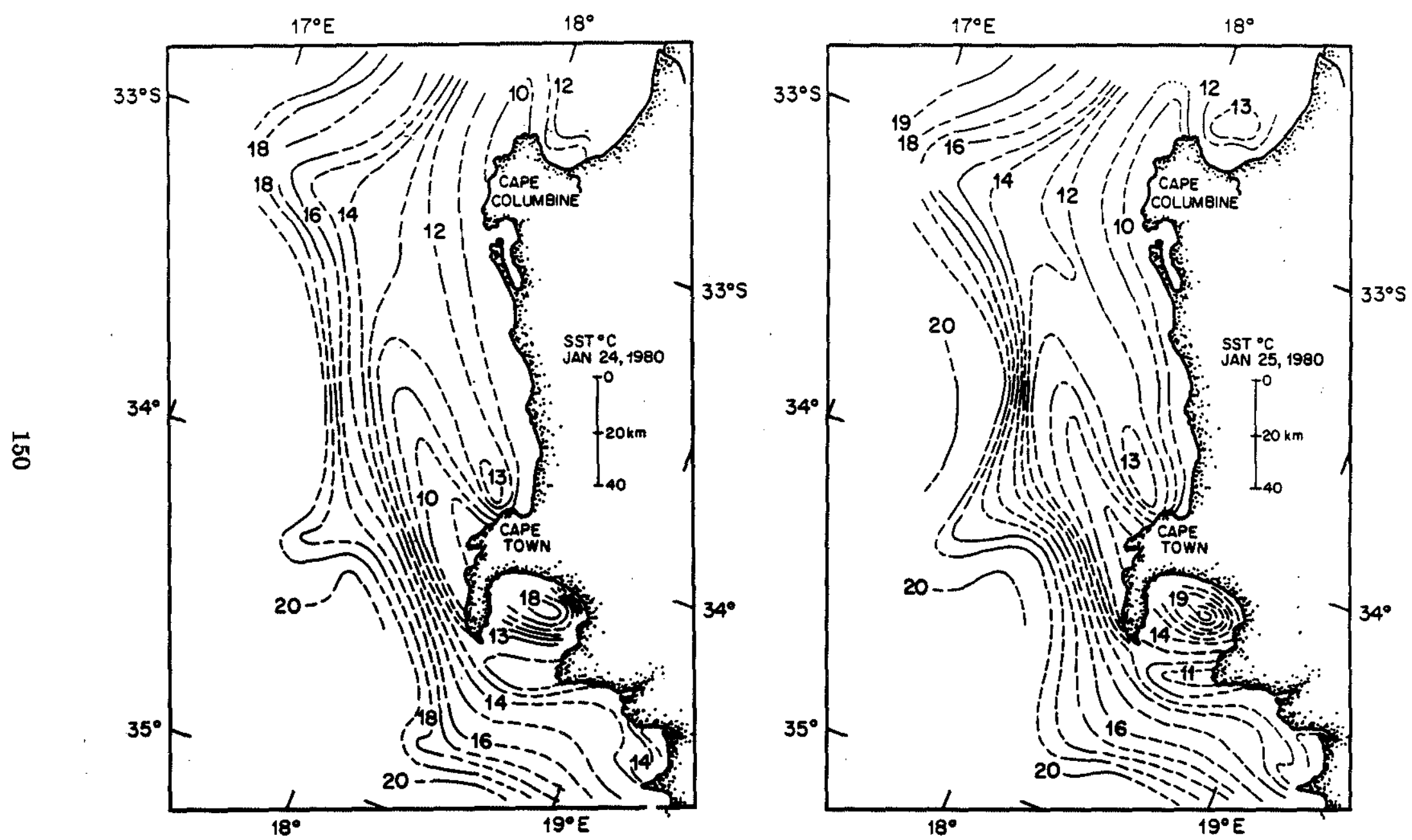
the bottom topography restricts their movement cross-shelf. Again, there is some propagation of the meander pattern to the northwest. The final SST map in this case study is from January 25 (Fig. 6.12d). Growth in the alongfront meander is evident, but the pattern has not shifted in the alongfront direction significantly. Note the sharpening of the surface front at the inshore peaks especially near $33^{\circ} 45^{\prime} \mathrm{S}$. This apparently occurs because the cross-shelf movement of the inshore meander peaks is restricted by the topography resulting in a compression of the surface isotherms. There is some evidence that the wave is steeper downstream of regions where the upper layer is shallow (e.g. the peak near $34^{\circ} 15^{\prime} \mathrm{S}$ ). This pattern is consistent with the wave motion in the unstable mode found from the simple stability model as discussed in section 6.2 above. It should be noted that wave steepening is a nonlinear effect, but the predictions of the linear wave motion suggest that this effect will occur.

As in the previous studies off Oregon and Nova Scotia, quantitative estimates can be made for the properties of the observed waves described qualitatively above. The results are, where individual estimates used are given in Table 6.3,

$$
\begin{aligned}
\text { alongfront wavelength } & \sim 81-150 \mathrm{~km}, \\
\text { phase speed } & \sim 10 \mathrm{~cm} \mathrm{~s}^{-1} \text { to the northwest, } \\
\text { e-folding time } & \sim 6 \text { days. }
\end{aligned}
$$

To obtain predictions for the same properties, the simple stability model is run with bottom topography from a cross-shelf section originating near $33^{\circ} 35^{\prime} \mathrm{S}$ (Fig. 6.13a). The bottom slope parameter $s$ is shown as a function of cross-shelf distance in Fig. 6.13b. No subsurface density information is available during the time the SST maps discussed above were calculated. To get a value of $H_{1}$ and $\delta$ for input into the model, vertical sections through similar upwelling fronts during the same season but for a different year are used (Bang, 1973). The values used are $H_{1}=70 \mathrm{~m}$ and $\delta=0.0015$ which lead to a Rossby radius of $12.4 \mathrm{~km}$ and a long internal gravity wave phase 

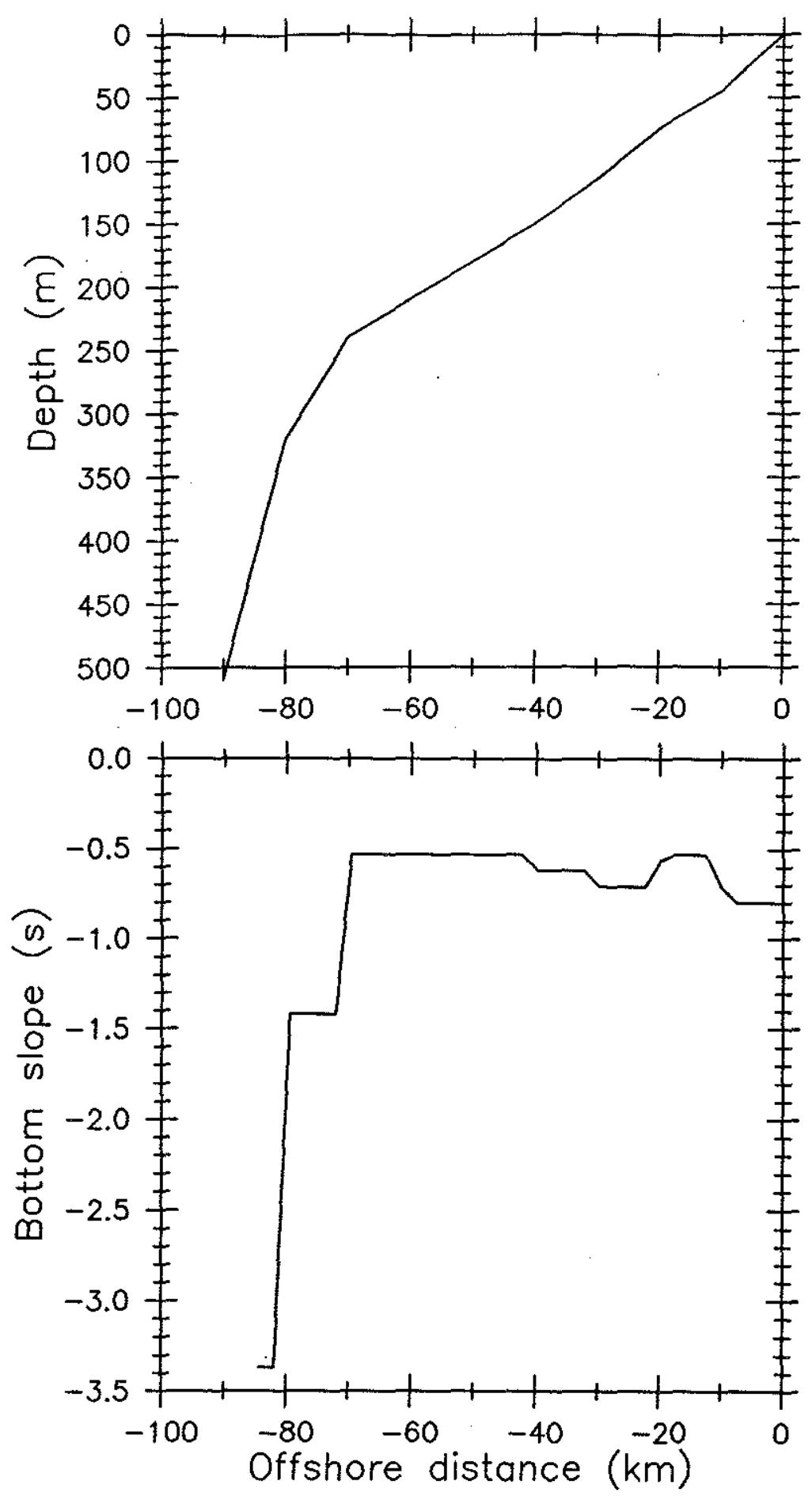

Figure 6.13: (a) Bottom topography off the southwest coast of Africa used in the stability model. (b) Bottom slope parameter $s$ for the cross-shelf topography in (a). 
speed of $101 \mathrm{~cm} \mathrm{~s}^{-1}$. Again, the interface parameter $\alpha$ is chosen equal to one as a reasonable first guess. From the aircraft SST maps (Fig. 6.12) the front is $\sim 40-50 \mathrm{~km}$ offshore which, given the bottom topography displayed in Fig. 6.13 , places the front in $150-170 \mathrm{~m}$ of water. The properties of the fastest growing waves using the above model inputs and varying the offshore position of the surface front are

$$
\begin{array}{cl}
\text { alongfront wavelength } & 111-120 \mathrm{~km}, \\
\text { phase speed } & 1-2 \mathrm{~cm} \mathrm{~s}^{-1} \text { to the northwest, } \\
\text { e-folding time } & 14-16 \text { days. }
\end{array}
$$

While the predicted alongfront scale compares reasonably well with the observed values, the phase speed and growth rate are too slow. Both these values could be increased by increasing the steepness of the interface profile (i.e. making the overall vertical shear near the surface front larger). As in the previous case studies, the presence of underlying stratification, an effect omitted in the present stability model, may isolate the upwelling front from the stabilizing influence of a sloping bottom.

To assess the influence of bottom friction on the stability properties of the system, a comparison between the e-folding time of the fastest growing wave and the frictional damping time scale can be made. Using a value of $\lambda_{*}=0.04 \mathrm{~cm} \mathrm{~s}^{-1}$, the frictional time scale is estimated as $\sim 14$ days. This time scale is comparable to the e-folding times found above, so the possibility of destabilization by friction, as discussed in section 5.5 , is possible. Results from a viscous model using the above value of $\lambda_{*}$ yield the following wave properties

$$
\begin{array}{cl}
\text { alongfront wavelength } & 85-92 \mathrm{~km}, \\
\text { phase speed } & 5-10 \mathrm{~cm} \mathrm{~s}^{-1} \text { to the northwest, } \\
\text { e-folding time } & 8-10 \text { days. }
\end{array}
$$

Linearized bottom friction does improve the model estimates of phase speed and growth rate, but the model still underpredicts the growth rate. In 
summary, even in the presence of frictional damping the simple stability model predicts unstable wave properties in the range of observed variability.

One difference between this case study and the previous observations off Oregon and Nova Scotia is the presence of a constant upwelling favorable wind stress throughout the observation period. In the previous case studies, upwelling favorable winds occur in events separated by periods of weak or downwelling favorable winds. The simple stability model developed here does not include wind stress and should, therefore, only be compared to observations obtained during these periods of weak winds between upwelling events. However, as discussed in the beginning of chapter 3 , the stability model may apply equally well to basic state flows which exist as a result of a steady-state balance between wind forcing and dissipation. In other words, these two processes will not enter the stability analysis other than through their effects on the basic state flow field. This case study off southwestern Africa suggests that the simple model may be used to analyze the stability of a coastal upwelling front in the presence of wind stress. The introduction of time-dependence in the basic state flow field from the action of wind stress may, however, affect the stability of the front. This point is discussed further in chapter 7 below.

A final caveat that should be mentioned, which applies to all the observational comparisons, is that the observed meanders are finite amplitude phenomena while the model predictions are formally valid only at small amplitude. Nevertheless, given the inaccuracies in specifying the basic state flow field as well as the model limitations, the alongfront variability observed on coastal upwelling fronts off the southwest tip of Africa can be interpreted in terms of unstable frontal waves. 


\subsection{Summary}

Observations from three regions of active upwelling show the existence of alongfront variability on coastal upwelling fronts. The properties of the observed wavelike disturbances and the predictions from the simple twolayer stability model are in reasonable agreement. Although the observed meanders are finite amplitude, they still appear to be growing which suggests that the predicted growth from the linear theory may continue as the flow becomes nonlinear. The model prediction is best for the alongfront scale of the meanders. Discrepancies between the model estimates and the observations can be due to several factors. Inaccuracies in specifying the basic state flow due to limited subsurface density information will lead to differences between observed and modelled wave properties. There are also a number of omissions in the present stability model (e.g. no wind stress) which are definitely present in the observed upwelling systems. These omissions will be discussed further in the next chapter. However, the fact that the simple stability model presented here predicts wave properties in the range of the observed values, supports the idea that alongfront variability can be interpreted in terms of unstable frontal waves. 


\begin{tabular}{|c|c|c|c|}
\hline Date & $\begin{array}{c}\text { Wavelength } \\
\text { estimates }(\mathrm{km})\end{array}$ & $\begin{array}{c}\text { Phase speed } \\
\text { estimates }\left(\mathrm{cm} \mathrm{s}^{-1}\right)\end{array}$ & $\begin{array}{c}\text { cross-front } \\
\text { peak-to-peak } \\
\text { amplitude estimates }(\mathrm{km})\end{array}$ \\
\hline $1 / 22 / 80$ & 125 & & $4,9,10$ \\
\hline & & $5,16,6,6,9,12$ & $11,2,14,16,4$ \\
\hline $1 / 23 / 80$ & 150,81 & & $6,2,10,5,7$ \\
\hline & & $54,9,34,21$ & \\
\hline $1 / 24 / 80$ & 132,95 & & $12,8,11,11,20,16$ \\
\hline & 125,90 & $-29,2,-26,4$ & \\
\hline \hline
\end{tabular}

Table 6.3: Estimates of the properties of observed alongfront variability off the southwest coast of Africa. 


\section{Chapter 7}

\section{Discussion}

\subsection{Introduction}

In the previous chapters the existence of unstable waves on model coastal upwelling fronts has been demonstrated. Observations of alongfront variability from SST maps of the coastal ocean provide evidence for the existence of these unstable waves in nature. A more detailed connection between the stability model and the real ocean is difficult because of the omissions made in simplifying the model. The first part of this chapter will be a discussion of these omissions, concentrating on the possible weaknesses of the present model. Possible future modelling efforts will be outlined which include the more important processes omitted in the present study. Following this, the possible connection between the finite amplitude form of the unstable frontal waves and the cross-shelf flux of water properties will be commented on.

\subsection{Simplifications and Omissions}

Some of the simplifications made in the stability model presented here are potentially more serious than others. The less troublesome simplifications will be addressed first, followed by a look at three of the more serious omis- 
sions beginning with the small amplitude restriction of the model. Modelling the density structure associated with a coastal upwelling front as two homogeneous layers can be criticized for several reasons. Layer models are usually employed to simplify the analysis, but severely restrict the vertical resolution of motion in the system. This restriction is certainly present in the model developed here. Further, since the interface rises to the surface, lack of vertical structure in the density field necessarily becomes lack of horizontal structure in the surface front. Vertical sections of density across coastal upwelling fronts (Fig. 1.1) show a thin region of compressed isopycnals. Thus, modelling this region as a sharp interface between layers is reasonably realistic. Representing the water column beneath the interface as a single homogeneous layer omits the possible influence of the underlying stratification apparent in Fig. 1.1. Since the motion of the unstable mode discussed in chapter 5 predominates in the upper layer, the density structure beneath the interface is not expected to affect greatly the wave's properties. However, as discussed in section 5.4 and chapter 6 , the underlying stratification may isolate the upper water column from the influence of the bottom. As a result, a sloping bottom may not stabilize the front to the degree it does with a homogeneous lower layer. In addition, the damping effect of bottom friction may be reduced. The result of both these effects is that the front may be more unstable than predicted here. In addition, an increase in the wave propagation speed in the direction of the basic state flow would result. The underprediction of phase speed and growth rate by the model when applied to observed frontal meanders is discussed in chapter 6.

Layer models in the presence of finite bottom topography have also been criticized for their incorrect description of the topographic vorticity waves present in the system (Chapman, 1983). This problem is not anticipated to affect the instability results presented here because the stable modes involved in the unstable resonance are frontally-trapped not 
coastally-trapped.

The lack of cross-frontal mixing or interfacial friction which may lead to mixing is another weakness of the model. These processes are likely to be important in the formation and evolution of a coastal upwelling front. The removal of energy from the upper layer via interfacial friction and the degradation of the sharp interface and surface front by mixing would most likely lead to a decrease in the growth rates of the unstable waves predicted by this simple model. Observations of frontal variability suggest that these processes do not completely quench the unstable waves.

Linear instability theory is restricted to describing the small amplitude behavior of unstable waves. A nonlinear calculation is formally required to describe the evolution of the instability once it reaches finite amplitude. Sometimes, waves which grow exponentially with time at small amplitude stop growing or go into limit cycle oscillations upon reaching finite amplitude. The reader is directed to Pedlosky (1986) for a description of these processes and a reference list. The small amplitude unstable frontal waves found here may be subject to this type of behavior after the initial period of predicted exponential growth. However, observations show that meanders in the coastal upwelling front can grow to finite amplitude and even break into detached eddies. Since these features have scales comparable to those predicted here, it is believed that the linear, small amplitude theory provides results which are likely to hold up, at least qualitatively, at later times as the flow becomes nonlinear.

A potentially fruitful way to study the finite amplitude behavior of these unstable waves is to use a fully nonlinear, time-dependent numerical model of a coastal upwelling front. A model with a number of homogeneous layers of different density or one with continuous stratification would be appropriate. The coastal upwelling front could be established by specifying the basic state velocity and density fields such that they are in geostrophic balance. This could be problematic, since a small amount of noise (present in any 
numerical model) would upset this equilibrium and lead to transient circulations as the fluid adjusts to geostrophic balance. This may complicate the stability analysis. Another way to establish the coastal upwelling front is to spin up the model from a state of rest by applying an alongshore wind stress. Once the front is established, a perturbation of a specified scale could be imposed. The time-dependent flow field could then be analyzed at later times looking for growth or decay of this perturbation. A series of experiments of this nature can be carried out to detail the finite amplitude behavior of the unstable frontal waves.

Another possible weakness of the model presented here is the lack of wind stress. As described in chapter 2, an alongshore wind stress is essential to the formation of a coastal upwelling front. The stability model presented here takes the fully developed, wind-formed front as its starting point. Some observational evidence suggests that coastal upwelling fronts are more stable (i.e. two-dimensional) in the presence of a strong, steady wind (Curtin, 1979). After the cessation, reversal or weakening of a wind event, the front is observed to meander. Other observations, such as those presented here off the southwest coast of Africa, show that alongfront meanders can exist in the presence of a steady wind. The laboratory models of Narimousa and Maxworthy (1985) include a steady wind stress which creates a coastal upwelling front. They observe unstable waves for all values of the wind stress. The time between the onset of the surface stress and the appearance of unstable waves is shorter for higher values of the wind stress. This is most likely due to the increased vertical shear driven by the stronger wind. Comparison of Narimousa and Maxworthy's (1985) laboratory models with coastal upwelling fronts in nature is suspect since their experiments reach an equilibrium state while an actual upwelling front will migrate continually offshore under the effect of a steady upwelling-favorable wind stress and in the absence of dissipation. The above observational and experimental evidence does not establish clearly the effect of wind stress 
on the stability of a coastal upwelling front. The present study also does not answer this important question.

The stability properties of the front may be affected by changes in the basic state flow field which arise due to wind forcing. Specifically, a steady wind stress will drive an upwelling circulation which depends on time until a steady-state balance between the forcing and dissipation is established. Prior to the steady state, the alongshore flow speed will increase with time and the surface front will migrate offshore. Time-dependence in the basic state flow will also result from the action of a time-dependent wind stress. The stability model presented here employs a fixed basic state flow. To model the effect of a wind stress realistically, a model with a time-dependent basic state flow should be developed. A wind stress may also affect the stability of a coastal upwelling front through the interaction of the winddriven Ekman flow with the front. To model this potential effect, motions in each of the various Ekman layers must be resolved. Offshore Ekman flux in the upper part of the water column, flow in Ekman layers adjacent to the upwelled interface and motion within an Ekman layer at the ocean bottom must all be included. Analytic solutions to these problems would be quite complicated. Progress could be made on this problem through the use of a numerical model. As discussed above, an alongshore wind stress could be used in a numerical model to establish a coastal upwelling front. The growth or decay of imposed perturbations could then be studied in the presence of a steady alongshore wind stress. Alternatively, the wind stress could be weakened, reversed or removed completely and the stability of the ensuing flow analyzed. A series of experiments of this type could answer the question posed above.

A final major weakness of the model, as alluded to in chapter 6 in a discussion of observed frontal variability, is the lack of alongshore variability in the bottom topography and/or the shoreline configuration. In chapter 5 , the profound influence of the local lower layer depth and the bottom 
slope on the properties of the unstable waves was presented. Surely the stability of the upwelling front will be different as the alongshore jet flows between regions of different topography. The theory presented here is valid for wave motions with alongshore scales much smaller than the alongshore scale of the topographic variability. This may hold true in some regions of active upwelling and the comparisons between the model predictions and observations is legitimate. However, other observations have suggested that alongshore topographic variations are important to coastal circulation. Studies off the northern California shelf have suggested that regions near capes and points are areas of enhanced upwelling (Kelly, 1985) and vigorous cross-shelf velocities (Davis, 1985). How does the alongshore topographic variability affect the stability properties of a coastal upwelling front? Does the topography create local regions where instability is more likely? Another possibility is that the scale of the topographic variability may influence the scale of the unstable frontal waves. To address these questions, the periodic dependence on $y$ of the wave form must be abandoned or the topographic variations may be made periodic in $y$ in which case the differential equations will have coefficients which depend on $y$. Either case leads to a system which is difficult to solve analytically. Perhaps the best solution is to analyze a numerical model of a coastal upwelling front as discussed above. A series of experiments with different alongshore bottom topographies and/or coastline variations would help to resolve the questions raised above.

Even with the omissions discussed above, the simple stability model presented here predicts the existence of unstable waves with properties in the range of observed variability. It is believed that any future numerical modelling efforts would serve to refine, but not refute, the processes and results presented here. 


\subsection{Implications}

The unstable frontal waves discussed in this study could have an important effect on the circulation associated with a coastal upwelling front. As mentioned briefly in the introduction, the wave may grow to finite amplitude and redirect the normally alongshore flow in the cross-shelf direction. The waves may grow large enough to form detached eddies which remain on the shelf disrupting the alongshore flow. Some observational evidence for this behavior was presented in chapter 6. Another possibility is that the finite amplitude wave peaks away from the coast may grow in the offshore direction until they disrupt the alongshore flow and create strong flows off the shelf. This would serve to transport large amounts of upwelled water off the shelf. These so-called offshore jets or squirts have been documented in a number of studies off the west coast of the United States (e.g. Davis, 1985; Kosro, 1987). The redirection of the normally alongshore flow by large amplitude unstable frontal waves is only one possible explanation for the existence of these strong, narrow, offshore flows. While other mechanisms involving variations in alongshore bottom topography, and/or coastline configuration and/or wind stress have been proposed (Hartwig and Brink, 1985), the instability of the flow field associated with the coastal upwelling front remains a likely candidate. Currently, an observational and theoretical research program (Coastal Transition Zone) is taking place to help elucidate the processes which lead to the formation and evolution of these offshore squirts of cold, upwelled water.

Unstable waves on coastal upwelling fronts may also contribute to the cross-shelf eddy flux of water properties. In their study of the heat budget off the coast of Oregon in 1973, Bryden et al. (1980) found that the crossshore eddy heat flux is an important process. They note that the eddy heat flux is directed down the mean horizontal temperature gradient and thus removes potential energy from the mean circulation. This behavior is 
consistent with the unstable waves discussed here which gain energy from the mean circulation via baroclinic instability. The presence of a coastal upwelling front during the time period of their study (Figs. 1.1, 6.2 and 6.3), lends further support to the possibility that the eddy flux came from the type of instability modelled here. Since Bryden et al. (1980) only analyzed data from one current meter mooring they were unable to estimate an alongshore scale for the eddy motions. Recently, Lentz (1987), using data from three alongshore moorings deployed as part of the Coastal Ocean Dynamics Experiment (CODE), found alongshore variability in the cross-shelf eddy heat flux with alongshore scales less than $56 \mathrm{~km}$. This alongshore scale provides an upper bound to compare to the size of the finite amplitude eddies which may evolve from the unstable frontal waves modelled here. That these eddy motions may be due to instability associated with the coastal upwelling front is further corroborated by the presence of the front near the moorings during the analysis period of the above study (Lentz, 1987). Davis (1985), using drifter data from CODE, also found vigorous eddy variability on scales of $40 \mathrm{~km}$ or less. Neither of the above two studies clearly establishes a lower bound for the alongshore scale of the eddy variability. The above studies indicate the importance of cross-shelf eddy heat flux to the heat budget for an area of active upwelling. The cross-shelf eddy flux of other water properties (e.g. nutrients) is also of practical importance. The unstable waves discussed here provide a mechanism for the existence of these eddy fluxes. 


\section{Chapter 8}

\section{Conclusions}

This study of a coastal upwelling front over topography shows that observed alongfront variability can often be explained in terms of an instability process. An unstable mode is described which gains energy from the basic state flow associated with the upwelling front via baroclinic instability. This wave amplifies in the absence of any external forcing. The properties of the fastest growing unstable wave in the system are in the range of observed alongfront variability.

The simple two-layer model developed here uses the geostrophic momentum approximation applied to the shallow water equations. This system allows the presence of a surface front, large horizontal shears and strong bottom slopes, features which are not able to be represented in a model using quasi-geostrophic dynamics. Allowing divergent flow introduces a term in the conservation of wave energy which is not positive definite (see chapter 4). The presence of this term allows the growth of unstable disturbances with positive, zero or negative wave energy. In the absence of external forcing, the total disturbance energy (the wave energy plus the change in the mean energy due to the presence of the unstable disturbance with the latter contribution hereafter called the mean energy) must be zero. Therefore, if the wave energy is positive then the mean energy must be negative and vice versa. The other possibility is that both the wave and mean 
energies are identically zero. In other words, an unstable wave can grow while exchanging energy with the mean flow so that the net change in the energy of the system remains zero. The nonintuitive idea of the growth of a wave with zero or negative energy can also be rationalized in terms of the exchange of disturbance energies between two stable modes whose alliance creates the unstable wave.

By combining the various conservation statements for the global properties of the system (potential vorticity, energy, momentum), a stability theorem is established in chapter 4 which allows the a priori determination of the stability of a coastal upwelling front. This theorem is basically a two-layer version of Ripa's (1983) theorem. The theorem differs from the traditional quasi-geostrophic theorem by including an additional constraint on the basic state flow in order to insure stability. This additional constraint can be attributed directly to the presence of the term which is not positive definite in the definition of wave energy. The theorem successfully indicates the stability or possible instability of previous frontal models and of the flows associated with the coastal upwelling front of interest here.

An unstable wave on a coastal upwelling front over a flat bottom next to a coastal wall is described in detail in chapter 5. The structure of the unstable wave is dominant in the upper layer and is trapped within $\sim 1$ Rossby radius of the surface front. The upper layer flow field is partly ageostrophic. The lower layer flow is weak, more geostrophic and consists of closed cells of circulation beneath the surface front. The unstable wave propagates phase in the direction of the basic state flow. The energy conservation statements involving the new term, which is not positive definite, are confirmed by direct numerical evaluation using the solutions to the generalized algebraic eigenvalue problem. The effect of varying the model parameters is presented. Moving the front closer than $\sim 2$ Rossby radii to the coastal wall results in a decrease (by a maximum of $\sim 34 \%$ when the surface front lies immediately adjacent to the coastal barrier) in the growth 
rate of the fastest growing wave. Increasing the overall vertical shear of the basic state flow, by either decreasing the lower layer depth or increasing the steepness of the interface, results in an increase in the growth rate of the fastest growing wave. The effect of changing the lower layer depth is particularly dramatic, leading to complete stability for uniform potential vorticity flows over an infinitely deep lower layer.

Since realistic coastal upwelling fronts occur over continental shelves, the effect of sloping bottom topography on the stability of the system is investigated. A model with a linear bottom slope indicates that a bottom sloping in the same sense as the interface decreases the growth rates of the unstable waves in the system. The sloping bottom also decreases the alongfront wavenumber (increases the alongfront scale) of the fastest growing wave. When the bottom slope exceeds the maximum slope of the interface, the flow is completely stabilized. This result is the same as in traditional quasi-geostrophic theory. However, this complete quenching of the unstable frontal waves is not likely to be relevant to realistic coastal upwelling fronts for two reasons. First, continental shelves contain regions of varying bottom slope so that somewhere the interface will slope more steeply than the bottom. This result is verified in a comparison of a model with arbitrary cross-shelf bottom topography to observed alongfront variability (chapter 6). Second, the presence of any density structure (either additonal layers or continuous stratification) beneath the interface may insulate the upper part of the water column from the effects of a sloping bottom. The present model excludes this effect since the lower layer is assumed homogeneous. In summary, for realistic coastal upwelling fronts the presence of sloping bottom topography decreases the growth rates of the unstable waves and increases the alongfront scale of the fastest growing mode, but is not expected, in general, to completely quench the instability.

The importance of bottom friction in coastal circulation motivates its inclusion in the present stability model. When the e-folding time scale of 
the unstable wave is short compared to the frictional damping time scale, bottom friction decreases the growth rates of the unstable waves by extracting energy from the wave. The presence of bottom friction also introduces a slowly growing mode at high wavenumbers due to the additional vorticity source. Since the fastest growing unstable mode is strongest in the upper layer, the presence of the lower layer insulates the wave from the influence of bottom friction. Bottom friction may also destabilize flows which are otherwise stable in the absence of dissipation, but the resulting unstable modes will have very small growth rates. This result is analogous to the destabilization of quasi-geostrophic flows by friction in the presence of the $\beta$ effect. The main result for typical coastal upwelling fronts is that bottom friction will not completely quench the unstable frontal waves. Other forms of dissipation (e.g. interfacial friction) might possibly have larger effects, but observations of alongfront variability suggest that unstable frontal waves are not eliminated by their presence.

The predictions for the properties of the fastest growing waves from the simple two-layer model are within the range of observed alongfront variability from several areas of active upwelling as described in chapter 6. Even though the model is only formally valid for small amplitude disturbances and the observed motions are definitely finite amplitude, the model predictions are in fair agreement with observed properties. A more detailed comparison would require refinement of the present stability model. Three possible improvements are the extension to finite amplitude, the inclusion of wind stress (and thus a time-dependent basic state flow) and alongshore topographic variations. A potentially successful way to study these effects is to use a full nonlinear numerical model of a coastal upwelling front. However, even without these refinements, the results presented here suggest that observed alongfront variability in the coastal ocean can often be interpreted in terms of the instability of a coastal upwelling front. 


\section{Appendix A}

This appendix contains the algebraic details of the derivations of many of the conservation statements in section 4.2.

\section{Conservation of Potential Vorticity}

The derivation of the conservation of potential vorticity in each layer from the full, nonlinear geostrophic momentum equations (3.5) and (3.6) is as follows. Note the differences from the traditional shallow water equation development (Pedlosky, 1986). Details of the derivation for the upper layer are presented with the lower layer derivation following in an analogous manner. Taking the curl of the upper layer momentum equations $[\partial(3.5 a) / \partial y+\partial(3.5 b) / \partial x]$ yields

$$
\frac{D}{D t}\left(\zeta_{1_{x z}}+\zeta_{1_{y y}}\right)+\left(u_{1_{x}}+v_{1_{y}}\right)+\left(v_{1_{x}}+u_{1_{y}}\right) \zeta_{1_{x y}}+u_{1_{x}} \zeta_{1_{x x}}+v_{1_{y}} \zeta_{1_{y y}}=0 .
$$

Rewriting (3.5c) as

$$
\left(u_{1_{x}}+v_{1_{y}}\right)=-\frac{1}{\left(h_{1}+\delta \zeta_{1}\right)} \frac{D}{D t}\left(h_{1}+\delta \zeta_{1}\right)
$$

allows $\left(u_{1_{x}}+v_{1_{y}}\right)$ to be replaced in (A.1) to yield

$$
\begin{gathered}
\frac{D}{D t}\left(\zeta_{1_{x x}}+\zeta_{1_{y y}}\right)-\frac{1}{\left(h_{1}+\delta \zeta_{1}\right)} \frac{D}{D t}\left(h_{1}+\delta \zeta_{1}\right)+ \\
\left(v_{1_{x}}+u_{1 y}\right) \zeta_{1_{x y}}+u_{1_{x}} \zeta_{1_{x x}}+v_{1_{y}} \zeta_{1_{y y}}=0
\end{gathered}
$$

To replace the term in (A.3) involving $\left(v_{1_{x}}+u_{1_{y}}\right)$ take $\partial(3.5 a) / \partial x$ and add to $\partial(3.5 b) / \partial y$ to obtain

$$
2 \frac{D}{D t} \zeta_{1_{x y}}+\left(v_{1 x}+u_{1 y}\right)+\left(u_{1 y}+v_{1 y}\right) \zeta_{1_{z y}}+\left(v_{1_{x}}+1\right) \zeta_{1_{y y}}+\left(u_{1 y}-1\right) \zeta_{1_{x x}}=0
$$


Substitute for $\left(u_{1_{z}}+v_{1_{y}}\right)$ from (A.2) and rearrange to get

$$
\begin{aligned}
\left(v_{1 x}+u_{1_{y}}\right)= & -2 \frac{D}{D t} \zeta_{\mathbf{1}_{z y}}+\frac{\zeta_{1_{z y}}}{\left(h_{1}+\delta \zeta_{1}\right)} \frac{D}{D t}\left(h_{1}+\delta \zeta_{1}\right)- \\
& \left(v_{\mathbf{1}_{z}}+1\right) \zeta_{\mathbf{1}_{\mathbf{y}}}-\left(u_{\mathbf{1}_{y}}-1\right) \zeta_{\mathbf{1}_{x \boldsymbol{x}}} .
\end{aligned}
$$

Multiplying (A.4) by $S_{\mathbf{z}_{\mathbf{z}}}$ and substituting in (A.3) yields

$$
\begin{aligned}
& \frac{D}{D t}\left(\zeta_{1_{x z}}+\zeta_{1_{y y}}-\zeta_{1_{x y}}^{2}\right)+\frac{\left(\zeta_{1_{z y}}^{2}-1\right)}{\left(h_{1}+\delta \zeta_{1}\right)} \frac{D}{D t}\left(h_{1}+\delta \zeta_{1}\right)+ \\
& \quad \quad_{1_{x z}}\left[u_{1_{z}}-\zeta_{1_{z y}}\left(u_{1 y}-1\right)\right]+\zeta_{1_{y y}}\left[v_{1_{y}}-\zeta_{1_{x y}}\left(v_{1_{z}}+1\right)\right]=0 .
\end{aligned}
$$

Finally, the last two groups of terms in square brackets are replaced using $\zeta_{1_{x z}}$ times (3.5a) plus $\zeta_{1_{y y}}$ times $(3.5 b)$ or

$$
\begin{aligned}
\frac{D}{D t}\left(\zeta_{1_{z x}} \zeta_{1_{y y}}\right)+ & \zeta_{1_{x z}}\left[v_{1_{y}}+\left(u_{1_{y}}-1\right) \zeta_{1_{x y}}+v_{1_{y}} \zeta_{1_{y y}}\right]+ \\
& \zeta_{1_{y y}}\left[u_{1_{z}}+\left(v_{1_{z}}+1\right) \zeta_{1_{x y}}+u_{1_{x}} \zeta_{1_{x y}}\right]=0 .
\end{aligned}
$$

As before, (A.2) is used to replace terms proportional to $\left(u_{1_{x}}+v_{1_{y}}\right)$ with the result

$$
\begin{gathered}
\frac{D}{D t}\left(\zeta_{1_{z x}} \zeta_{1_{y y}}\right)-\left(\zeta_{1_{z z}} \zeta_{1_{y y}}+\zeta_{1_{x z}}+\zeta_{1_{y y}}\right) \frac{1}{\left(h_{1}+\delta \zeta_{1}\right)} \frac{D}{D t}\left(h_{1}+\delta \zeta_{1}\right)- \\
\quad \zeta_{1_{z z}}\left[u_{1_{z}}-\zeta_{1_{z y}}\left(u_{1,}-1\right)\right]-\zeta_{1_{y y}}\left[v_{1_{y}}-\zeta_{1_{x y}}\left(v_{1_{z}}+1\right)\right]=0 .
\end{gathered}
$$

Adding (A.5) and (A.6) yields the final result

$$
\begin{aligned}
& \frac{D}{D t}\left(\zeta_{1_{x x}}+\zeta_{1_{y y}}-\zeta_{1_{z y}}^{2}+\zeta_{1_{z z}} \zeta_{1_{y y}}\right)- \\
& \quad\left(1+\zeta_{1_{x z}}+\zeta_{1_{y y}}-\zeta_{1_{z y}}^{2}+\zeta_{1_{z x}} \zeta_{1_{y y}}\right) \frac{1}{\left(h_{1}+\delta \zeta_{1}\right)} \frac{D}{D t}\left(h_{1}+\delta \zeta_{1}\right)=0
\end{aligned}
$$

or

$$
\frac{D}{D t}=\left(\frac{1+\zeta_{1_{z x}}+\zeta_{1_{y y}}-\zeta_{1_{x y}}^{2}+\zeta_{1_{x z}} \zeta_{1_{y y}}}{h_{1}+\delta \zeta_{1}}\right)=0
$$

from which (4.1a) and (4.1b) follow. The derivation of potential vorticity conservation in the lower layer follows from a similar manipulation of (3.6). 


\section{Conservation of Energy}

The derivation of the conservation of energy (4.3) begins by forming expressions involving the kinetic energy in each layer. Multiplying (3.5a) by $\left(h_{1}+\delta \zeta_{1}\right) \zeta_{1_{y}}$ and $(3.5 \mathrm{~b})$ by $\left(h_{1}+\delta \zeta_{1}\right) \zeta_{1_{x}}$ then adding yields

$$
\begin{gathered}
\left(h_{1}+\delta \zeta_{1}\right) \frac{D}{D t_{1}}\left(\frac{\zeta_{1_{x}}^{2}+\zeta_{1_{y}}^{2}}{2}\right)+h_{2} \frac{D}{D t_{2}}\left(\frac{\zeta_{T_{x}}^{2}+\zeta_{T_{y}}^{2}}{2}\right)= \\
-\left(h_{1}+\delta \zeta_{1}\right)\left(u_{1} \zeta_{1_{x}}+v_{1} \zeta_{1_{y}}\right)-h_{2}\left(u_{2} \zeta_{T_{x}}+v_{2} \zeta_{T_{y}}\right)
\end{gathered}
$$

where

$$
\frac{D}{D t_{i}}=\frac{D}{D t}+u_{i} \frac{\partial}{\partial x}+v_{i} \frac{\partial}{\partial y} \quad i=1,2 .
$$

Now, (3.5c) times $\left(\zeta_{1_{z}}^{2}+\zeta_{1_{y}}^{2}\right) / 2$ plus (3.6c) times $\left(\zeta_{T_{x}}^{2}+\zeta_{T_{y}}^{2}\right) / 2$ gives

$$
\begin{aligned}
& \left(\frac{\zeta_{1_{z}}^{2}+\zeta_{1_{y}}^{2}}{2}\right) \frac{\partial}{\partial t}\left(h_{1}+\delta \zeta_{1}\right)+ \\
& \quad\left(\frac{\zeta_{1_{z}}^{2}+\zeta_{1_{y}}^{2}}{2}\right)\left\{\left[u_{1}\left(h_{1}+\delta \zeta_{1}\right)\right]_{x}+\left[v_{1}\left(h_{1}+\delta \zeta_{1}\right)\right]_{y}\right\}+ \\
& \quad\left(\frac{\zeta_{T_{x}}^{2}+\zeta_{T_{y}}^{2}}{2}\right) \frac{\partial}{\partial t} h_{2}+\left(\frac{\zeta_{T_{z}}^{2}+\zeta_{T_{y}}^{2}}{2}\right)\left[\left(u_{2} h_{2}\right)_{x}+\left(v_{2} h_{2}\right)_{y}\right]=0 .
\end{aligned}
$$

Adding (A.7) and (A.8) yields

$$
\begin{aligned}
\frac{\partial}{\partial t} & {\left[\left(h_{1}+\delta \zeta_{1}\right)\left(\frac{\zeta_{1_{x}}^{2}+\zeta_{1_{y}}^{2}}{2}\right)+h_{2}\left(\frac{\zeta_{T_{z}}^{2}+\zeta_{T_{y}}^{2}}{2}\right)\right]+} \\
& \frac{\partial}{\partial x}\left[u_{1}\left(h_{1}+\delta \zeta_{1}\right)\left(\frac{\zeta_{1_{z}}^{2}+\zeta_{1_{y}}^{2}}{2}\right)+u_{2} h_{2}\left(\frac{\zeta_{T_{x}}^{2}+\zeta_{T_{y}}^{2}}{2}\right)\right]+ \\
& \frac{\partial}{\partial y}\left[v_{1}\left(h_{1}+\delta \zeta_{1}\right)\left(\frac{\zeta_{1_{x}}^{2}+\zeta_{1_{y}}^{2}}{2}\right)+v_{2} h_{2}\left(\frac{\zeta_{T_{x}}^{2}+\zeta_{T_{y}}^{2}}{2}\right)\right]= \\
& -\left(h_{1}+\delta \zeta_{1}\right)\left(u_{1} \zeta_{1_{z}}+v_{1} \zeta_{1_{y}}\right)-h_{2}\left(u_{2} \zeta_{T_{x}}+v_{2} \zeta_{T_{y}}\right) .
\end{aligned}
$$

The terms on the right-hand-side of (A.9) can be eliminated by forming an expression involving the potential energy. Multiplying (3.5c) by $\zeta_{1}$ and 
(3.6c) by $\zeta_{T}=(1-\delta) \zeta_{1}-h_{1}$ then adding gives

$$
\begin{aligned}
\frac{\partial}{\partial t}\left(\frac{h_{1}^{2}}{2}+\delta \zeta_{1} h_{1}\right)+ & \zeta_{1}\left[u_{1}\left(h_{1}+\delta \zeta_{1}\right)\right]_{x}+\zeta_{1}\left[v_{1}\left(h_{1}+\delta \zeta_{1}\right)\right]_{y}+ \\
& \zeta_{T}\left(u_{2} h_{2}\right)_{x}+\zeta_{T}\left(v_{2} h_{2}\right)_{y}=0 .
\end{aligned}
$$

Adding (A.9) and (A.10) yields

$$
\begin{gathered}
\frac{\partial}{\partial t}\left[\left(h_{1}+\delta \zeta_{1}\right)\left(\frac{\zeta_{1_{x}}^{2}+\zeta_{1_{y}}^{2}}{2}\right)+h_{2}\left(\frac{\zeta_{T_{x}}^{2}+\zeta_{T_{y}}^{2}}{2}\right)+\frac{h_{1}^{2}}{2}+\delta \zeta_{1} h_{1}\right]+ \\
\frac{\partial}{\partial x}\left[u_{1}\left(h_{1}+\delta \zeta_{1}\right)\left(\frac{\zeta_{1_{x}}^{2}+\zeta_{1_{y}}^{2}}{2}\right)+u_{2} h_{2}\left(\frac{\zeta_{T_{x}}^{2}+\zeta_{T_{y}}^{2}}{2}\right)+\right. \\
\left.\zeta_{1} u_{1}\left(h_{1}+\delta \zeta_{1}\right)+\zeta_{T_{2} u_{2} h_{2}}\right]+ \\
\frac{\partial}{\partial y}\left[v_{1}\left(h_{1}+\delta \zeta_{1}\right)\left(\frac{\zeta_{1_{x}}^{2}+\zeta_{1_{y}}^{2}}{2}\right)+v_{2} h_{2}\left(\frac{\zeta_{T_{x}}^{2}+\zeta_{T_{y}}^{2}}{2}\right)+\right. \\
\left.\zeta_{1} v_{1}\left(h_{1}+\delta \zeta_{1}\right)+\zeta_{T_{2} v_{2} h_{2}}\right]=0 .
\end{gathered}
$$

This expression is then averaged over one wavelength in $y$ and over the full $x$-domain. For the upper layer, the integration is carried out from $-\infty$ to the surface front. The integration in $x$ requires the application of boundary conditions which are discussed in detail in section 5.2. Basically, the crossfront velocity in each layer vanishes far from the surface front or, in the case of the lower layer, vanishes because of a coastal barrier. The final result is

$$
\frac{\partial}{\partial t} \int\left[\left(h_{1}+\delta \zeta_{1}\right)\left(\frac{\zeta_{1_{x}}^{2}+\zeta_{1_{y}}^{2}}{2}\right)+h_{2}\left(\frac{\zeta_{T_{x}}^{2}+\zeta_{T_{y}}^{2}}{2}\right)+\frac{h_{1}^{2}}{2}+\delta \zeta_{1} h_{1}\right] d a=0,
$$

where $\int(\quad) d a$ represents the integration described above. Equations (4.3) follow by neglecting terms of $0(\delta)$.

\section{Conservation of Momentum}

Derivation of the conservation of momentum (4.4) begins by taking (3.5b) times $\left(h_{1}+\delta \zeta_{1}\right)$ and adding to (3.5c) times $\zeta_{1_{x}}$ to obtain

$$
\frac{\partial}{\partial t}\left[\left(h_{1}+\delta \zeta_{1}\right) \zeta_{1_{s}}\right]+\frac{\partial}{\partial x}\left[\zeta_{1_{s}}\left(h_{1}+\delta \zeta_{1}\right) u_{1}\right]+
$$




$$
\frac{\partial}{\partial y}\left[\zeta_{1_{z}}\left(h_{1}+\delta \zeta_{1}\right) v_{1}\right]+\left(h_{1}+\delta \zeta_{1}\right) u_{1}=-\left(h_{1}+\delta \zeta_{1}\right) \zeta_{1_{y}}
$$

The final term on the left-hand-side can be written as

$$
\left(h_{1}+\delta \zeta_{1}\right) u_{1}=\frac{\partial}{\partial x}\left[\left(h_{1}+\delta \zeta_{1}\right) u_{1} x\right]-x \frac{\partial}{\partial x}\left[u_{1}\left(h_{1}+\delta \zeta_{1}\right)\right] .
$$

The last term in this expression can be replaced using (3.5c) times $x$

$$
x \frac{\partial}{\partial x}\left[u_{1}\left(h_{1}+\delta \zeta_{1}\right)\right]=-\frac{\partial}{\partial t}\left[x\left(h_{1}+\delta \zeta_{1}\right)\right]-\frac{\partial}{\partial y}\left[x v_{1}\left(h_{1}+\delta \zeta_{1}\right)\right]
$$

With these substitutions (A.11) becomes

$$
\begin{gathered}
\frac{\partial}{\partial t}\left[\left(h_{1}+\delta \zeta_{1}\right)\left(\zeta_{1_{x}}+x\right)\right]+\frac{\partial}{\partial x}\left[\left(\zeta_{1_{x}}+x\right)\left(h_{1}+\delta \zeta_{1}\right) u_{1}\right]_{x}+ \\
\frac{\partial}{\partial y}\left[\left(\zeta_{1_{x}}+x\right)\left(h_{1}+\delta \zeta_{1}\right) v_{1}\right]_{y}=-\left(h_{1}+\delta \zeta_{1}\right) \zeta_{1_{y}} .
\end{gathered}
$$

A similar manipulation for the lower layer yields

$$
\begin{gathered}
\frac{\partial}{\partial t}\left[\frac{h_{2}}{(1-\delta)}\left(\zeta_{T_{z}}+x\right)\right]+\frac{\partial}{\partial x}\left[\frac{h_{2} u_{2}}{(1-\delta)}\left(\zeta_{T_{x}}+x\right)\right]+ \\
\frac{\partial}{\partial y}\left[\frac{h_{2} v_{2}}{(1-\delta)}\left(\zeta_{T_{x}}+x\right)\right]=-h_{2} \zeta_{T_{y}}
\end{gathered}
$$

Adding (A.12) and (A.13) and then averaging over the domain of the fluid gives the final result

$$
\frac{\partial}{\partial t} \int\left[\left(h_{1}+\delta \zeta_{1}\right)\left(\zeta_{1_{s}}+x\right)+\frac{h_{2}}{(1-\delta)}\left(\zeta_{T_{x}}+x\right)\right] d a=0
$$

Equations (4.4) follow by ignoring terms of $0(\delta)$.

\section{Conservation of Wave Energy}

An expression for the time rate-of-change of the wave energy (4.9d) can be obtained directly from the linearized perturbation equations (3.8) and 
(3.9). Multiplying (3.8a) by $\overline{h_{1}} \zeta_{1_{y}}$ and (3.8b) by $\overline{h_{1}} \zeta_{1_{x}}$ then adding yields

$$
\begin{gathered}
\frac{\partial}{\partial t}\left[\overline{h_{1}}\left(\frac{\zeta_{1_{z}}^{2}+\zeta_{1_{y}}^{2}}{2}\right)\right]+\frac{\partial}{\partial x}\left[\overline{h_{1}} \bar{v}\left(\frac{\zeta_{1_{x}}^{2}+\zeta_{1_{y}}^{2}}{2}\right)\right]= \\
-\overline{h_{1}}\left(1+\bar{v}_{x}\right) u_{1} \zeta_{1_{x}}-\overline{h_{1}} v_{1} \zeta_{1_{y}} .
\end{gathered}
$$

A similar operation on the lower layer equations (3.9) yields

$$
\frac{\partial}{\partial t}\left[\overline{h_{2}}\left(\frac{\zeta_{T_{z}}^{2}+\zeta_{T_{y}}^{2}}{2}\right)\right]=-\overline{h_{2}}\left(u_{2} \zeta_{T_{z}}+v_{2} \zeta_{T_{y}}\right)
$$

To include the potential energy due to the displacement of the interface, multiply (3.8c) by $\zeta_{1}$ and (3.9c) by $\zeta_{T}$ then add to get

$$
\begin{gathered}
\frac{\partial}{\partial t}\left(\frac{\zeta_{2}^{2}}{2}\right)+\zeta_{1}\left(u_{1} \overline{h_{1}}\right)_{x}+\zeta_{1}\left[v_{1} \overline{h_{1}}-\bar{v} \zeta_{2}\right]_{y}+ \\
\zeta_{T}\left(u_{2} \overline{h_{2}}\right)_{x}+\zeta_{T}\left(v_{2} \overline{h_{2}}\right)_{y}=0,
\end{gathered}
$$

where terms of $0(\delta)$ have been ignored. Adding (A.14)-(A.16) yields

$$
\begin{aligned}
& \frac{\partial}{\partial t}\left[\overline{h_{1}}\left(\frac{\zeta_{1_{x}}^{2}+\zeta_{1_{y}}^{2}}{2}\right)+\overline{h_{2}}\left(\frac{\zeta_{T_{x}}^{2}+\zeta_{T_{y}}^{2}}{2}\right)+\frac{\zeta_{2}^{2}}{2}\right]+ \\
& \quad \frac{\partial}{\partial x}\left(\overline{h_{1}} u_{1} \zeta_{1}+\overline{h_{2}} u_{2} \zeta_{T}\right)+ \\
& \quad \frac{\partial}{\partial y}\left[\overline{h_{1}} \bar{v}\left(\frac{\zeta_{1_{x}}^{2}+\zeta_{1_{y}}^{2}}{2}\right)+\overline{h_{1}} v_{1} \zeta_{1}+\overline{h_{2}} v_{2} \zeta_{T}\right]=-\bar{v}_{x} \overline{h_{1}} u_{1} \zeta_{1_{x}}+\zeta_{1}\left(\bar{v} \zeta_{2}\right)_{y} .
\end{aligned}
$$

The cross-term appearing in the definition of wave energy (4.9d) is obtained by multiplying (3.8b) by $-\bar{v} \zeta_{2}$ and (3.8c) by $\bar{v} \zeta_{1_{x}}$ then adding. The result is

$$
\begin{gathered}
\left(-\bar{v} \zeta_{2} \zeta_{1_{z}}\right)_{t}-\left(1+\bar{v}_{x}\right) \overline{h_{1}} \bar{v} u_{1} \zeta_{2}+\bar{v} \zeta_{1_{x}}\left(u_{1} \overline{h_{1}}\right)_{x}+ \\
\bar{v} \overline{h_{1}} \zeta_{1_{z}} v_{1 y}-\bar{v}^{2}\left(\zeta_{1_{z}} \zeta_{2}\right)_{y}-\bar{v} \zeta_{2} \zeta_{1_{y}}=0
\end{gathered}
$$


Adding (A.18) to (A.17) yields

$$
\begin{aligned}
& \frac{\partial}{\partial t}\left[\overline{h_{1}}\left(\frac{\zeta_{1_{z}}^{2}+\zeta_{1_{y}}^{2}}{2}\right)-\bar{v} \zeta_{2} \zeta_{1_{z}}+\overline{h_{2}}\left(\frac{\zeta_{T_{x}}^{2}+\zeta_{T_{y}}^{2}}{2}\right)+\frac{\zeta_{2}^{2}}{2}\right]+ \\
& \frac{\partial}{\partial x}\left(\overline{h_{1}} u_{1} \zeta_{1}+\overline{h_{2}} u_{2} \zeta_{T}\right)+ \\
& \frac{\partial}{\partial y}\left[\overline{h_{1}} \bar{v}\left(\frac{\zeta_{1_{z}}^{2}+\zeta_{1_{y}}^{2}}{2}\right)+\overline{h_{1}} v_{1} \zeta_{1}+\overline{h_{2}} v_{2} \zeta_{T}-\bar{v}^{2} \zeta_{1_{z}} \zeta_{2}-\bar{v} \zeta_{2} \zeta_{1}\right]= \\
& -\bar{v}_{x} \overline{h_{1}} u_{1} \zeta_{1_{x}}+\left(1+\bar{v}_{x}\right) \overline{h_{1}} \bar{v} u_{1} \zeta_{2}-\bar{v} \zeta_{1_{x}}\left(u_{1} \overline{h_{1}}\right)_{x}-\bar{v} \overline{h_{1}} \zeta_{1_{x}} v_{1_{y}} .
\end{aligned}
$$

Integrating over the domain of the fluid and applying the same boundary conditions as used in the derivation of (4.3) and (4.4) results in

$$
\begin{aligned}
& \frac{\partial E_{2}}{\partial t}=-\int\left[\bar{h}_{1} \bar{v}_{x} u_{1} \zeta_{1_{z}}+\bar{v}^{2} u_{1} \zeta_{1_{x}}+\right. \\
& \left.\overline{h_{1}} \bar{v}\left(u_{1_{x}}+v_{1_{y}}\right) \zeta_{1_{x}}-\bar{v} u_{1} \zeta_{2}-\bar{v} \bar{v}_{x} u_{1} \zeta_{2}\right] d A
\end{aligned}
$$

which is equation (4.13).

\section{Displacement of the Surface Front}

As mentioned in section 4.2, the final term in (4.13) can be related to the changes in time of the displacement of the surface front. Consider the $O\left(\epsilon^{2}\right)$ upper layer continuity equation derived from (3.5c):

$$
\left(u_{1} \zeta_{2}\right)_{x}+\left(v_{1} \zeta_{2}\right)_{\nu}=0
$$

The $y$-average of this equation over one wavelength is

$$
\int\left(u_{1} \zeta_{2}\right)_{x} d y=0
$$

The final term in (4.13) can be written as

$$
-\int \frac{\bar{v}^{2}}{2} \int\left(u_{1} \zeta_{2}\right)_{x} d y d x+\left.\frac{\bar{v}^{2}}{2} \int u_{1} \zeta_{2}\right|_{x=-\infty} ^{x=x_{f}} d y
$$


From the $0\left(\epsilon^{2}\right) y$-averaged continuity equation the first term is zero. Since the disturbance will be assumed to be frontally-trapped (see section 5.2 for details), then $u_{1} \rightarrow 0$ as $x \rightarrow-\infty$ so that the second term is only evaluated at the front $x=x_{f}$. At the displaced position of the front $\left(x=x_{f}+\epsilon\right)$ the total depth vanishes

$$
h_{1}+\delta \zeta_{1}=0 \quad \text { at } \quad x=x_{f}+\epsilon .
$$

This can be expanded in a Taylor series to get an expression valid at the undisturbed frontal location

$$
\left.\left[(1-\delta) \overline{h_{1}}+\delta \overline{\zeta_{1}}+\zeta_{1}-\zeta_{T}\right]\right|_{x=x_{f}}+\left.\epsilon \frac{\partial}{\partial x}\left[(1-\delta) \overline{h_{1}}+\delta \overline{\zeta_{1}}+\zeta_{1}-\zeta_{T}\right]\right|_{x=x_{f}}=0
$$

but since $\overline{h_{1}}=\overline{\zeta_{1}}=0$ at $x=x_{f}$ and $\overline{h_{1 x}}=\overline{\zeta_{1 x}}=\bar{v}$ this reduces to the $0(\epsilon)$ expression

$$
\left(\zeta_{1}-\zeta_{T}\right)=-\bar{v} \epsilon \quad \text { at } \quad x=x_{f}
$$

Since $\left(\zeta_{1}-\zeta_{T}\right)=\delta \zeta_{1}-\zeta_{2}$ from (3.10) and if terms of $0(\delta)$ are ignored this can be written as

$$
\zeta_{2}=\bar{v} \epsilon \quad \text { at } \quad x=x_{f} .
$$

The velocity of the front in the $x$-direction can be written as

$$
u_{1}=\left(\frac{\partial}{\partial t}+\bar{v} \frac{\partial}{\partial y}\right) \epsilon
$$

Substituting (A.19) and (A.20) into the term evaluated at the front in the energy equation one obtains

$$
\begin{aligned}
\left.\frac{\bar{v}^{2}}{2} \int u_{1} \zeta_{2}\right|_{x=x_{f}} d y & =\left.\frac{\bar{v}^{3}}{2} \int\left[\left(\frac{\epsilon^{2}}{2}\right)_{t}+\bar{v}\left(\frac{\epsilon^{2}}{2}\right)_{y}\right]\right|_{x=x_{f}} d y \\
& =\frac{\partial}{\partial t}\left[\left.\frac{\bar{v}^{3}}{4} \int \epsilon^{2}\right|_{x=x_{f}} d y\right]
\end{aligned}
$$


Finally, this can be written on the left-hand-side which yields equation (4.14):

$$
\begin{aligned}
& \frac{\partial}{\partial t}\left[E_{2}-\left.\frac{\bar{v}^{3}}{4} \int \epsilon^{2}\right|_{x=x_{f}} d y\right]=-\int\left[\bar{h}_{1} \bar{v}_{x} u_{1} \zeta_{1_{x}}+\bar{v}^{2} u_{1} \zeta_{1_{z}}+\right. \\
& \left.\overline{h_{1}} \bar{v}\left(u_{1_{x}}+v_{1_{y}}\right) \zeta_{1_{x}}-\bar{v} u_{1} \zeta_{2}\right] d A .
\end{aligned}
$$

Derivation of $\partial E_{2} / \partial t \propto u_{1} q_{1}$

To relate $\partial E_{2} / \partial t$ to the cross-front eddy flux of perturbation potential vorticity, the Reynolds stress terms and baroclinic energy conversion term on the right-hand-side of (4.13) must be rewritten:

$$
\begin{aligned}
& \frac{\partial E_{2}}{\partial t}=\int\left[-\left(\overline{h_{1}} \bar{v}_{x} u_{1} \zeta_{1_{x}}+\bar{v}^{2} u_{1} \zeta_{1_{x}}+\overline{h_{1}} \bar{v} u_{1_{x}} \zeta_{1_{x}}\right)+\right. \\
& \left.\overline{h_{1}} \bar{v} u_{1} Q_{1} \zeta_{2}-\overline{h_{1}} \bar{v} v_{1_{y}} \zeta_{1_{x}}\right] d A \text {. }
\end{aligned}
$$

The term in parentheses can be rewritten as $\overline{h_{1}} \bar{v} u_{1} \zeta_{1_{x x}}$ because $\partial\left(\overline{h_{1}} \bar{v} u_{1} \zeta_{1_{z}}\right) / \partial x$ vanishes over the integral. To rewrite the final term in the integrand of (A.21), multiply (3.8a) by $\zeta_{1_{z y}}$ and (3.8b) by $\zeta_{1_{y y}}$ then add to obtain

$$
\zeta_{1_{x y}} \zeta_{1_{y t}}-\zeta_{1_{y y}} \zeta_{1_{x t}}+v_{1} \zeta_{1_{x y}}-\left(1+\bar{v}_{x}\right) u_{1} \zeta_{1_{y y}}=\frac{\partial}{\partial y}\left(\frac{\zeta_{1_{x}}^{2}+\zeta_{1_{y}}^{2}}{2}\right)
$$

Multiplying by $\overline{h_{1}} \bar{v}$ then averaging over the domain of the fluid yields

$$
\int \overline{h_{1}} \bar{v}\left[\zeta_{1_{y y}} \zeta_{1 y t}-\zeta_{1 y y} \zeta_{1 x t}+v_{1} \zeta_{1 x y}-\left(1+\bar{v}_{x}\right) u_{1} \zeta_{1 y y}\right] d A=0 .
$$

The first two terms may be replaced by

$$
\begin{aligned}
\int \overline{h_{1}} \bar{v} \zeta_{1_{z}} \zeta_{1_{y t}} d A & =-\int \overline{h_{1}} \bar{v} \zeta_{1_{z}} \zeta_{1_{y y t}} d A \\
-\int \overline{h_{1}} \bar{v} \zeta_{1_{y y}} \zeta_{1_{z t}} d A & =\int \overline{h_{1}} \bar{v}\left[-\left(\zeta_{1_{y y}} \zeta_{1_{z}}\right)_{t}+\zeta_{1_{z}} \zeta_{1_{y y t}}\right] d A,
\end{aligned}
$$


so (A.22) becomes

$$
\int \overline{h_{1}} \bar{v}\left[-\left(\zeta_{1_{y y}} \zeta_{1_{x}}\right)_{t}+v_{1} \zeta_{1_{x y}}-\left(1+\bar{v}_{x}\right) u_{1} \zeta_{1_{y y}}\right] d A=0
$$

Rearranging gives the desired result

$$
-\int \overline{h_{1}} \bar{v} v_{1_{y}} \zeta_{1_{z}} d A=\int \overline{h_{1}} \bar{v}\left[\left(1+\bar{v}_{x}\right) u_{1} \zeta_{1_{y y}}+\left(\zeta_{1_{y y}} \zeta_{1_{x}}\right)_{t}\right] d A
$$

Substituting into (A.21) yields

$$
\begin{aligned}
\frac{\partial E_{2}}{\partial t}= & \int{\overline{h_{1}}}^{2} \bar{v} u_{1}\left[\frac{\zeta_{1_{x x}}+\left(1+\bar{v}_{x}\right) \zeta_{1_{y y}}+Q_{1} \zeta_{2}}{\overline{h_{1}}}\right] d A+ \\
& \frac{\partial}{\partial t} \int \overline{h_{1}} \bar{v} \zeta_{1_{x}} \zeta_{1_{y y}} d A,
\end{aligned}
$$

which is just (4.16) since $q_{1}$ is given by (4.5c). [Note that a term of $0(\delta)$ in the definition of $q_{1}$ has been ignored.]

To understand why the final term in (4.16) exists, rewrite the definition of $q_{1}$ given by (4.5c) in a slightly different form

$$
q_{1}=\frac{\zeta_{1_{x x}}+\zeta_{1_{y y}}-Q_{1}\left(\zeta_{1}-\zeta_{T}\right)}{\bar{h}_{1}}+\frac{\bar{v}_{x} \zeta_{1_{y y}}}{\bar{h}_{1}} .
$$

The first group of terms is the exact geostrophic form of the perturbation potential vorticity. The final term represents part of the ageostrophic perturbation potential vorticity. To recognize this, the ageostrophic potential vorticity

$$
q_{1}^{a}=\frac{v_{1_{x}}^{a}-u_{1_{y}}^{a}}{\overline{h_{1}}}
$$

(where the superscript " $a$ " denotes an ageostrophic quantity) can be related to geostrophic quantities through the definitions of ageostrophic velocity obtained from (3.8a) and (3.8b). Substituting

$$
u_{1}^{a}=-\frac{1}{\left(1+\bar{v}_{x}\right)}\left(\bar{v}_{x} u_{1}^{g}+v_{1_{t}}^{g}+\bar{v} v_{1_{y}}^{g}\right)
$$


and

$$
v_{1}^{a}=u_{1_{t}}^{g}+\bar{v} u_{1 y}^{g}
$$

into the definition of $q_{1}^{a}$ yields

$$
q_{1}^{a}=\frac{\bar{v}_{x}}{\overline{h_{1}}\left(1+\bar{v}_{x}\right)}\left(u_{1_{y}}^{g}+u_{1_{x t}}^{g}+\bar{v} u_{1_{x y}}^{g}\right)
$$

Since $u_{1}^{g}=-\zeta_{1_{y}}$, the first term in the parentheses, multiplied by $\left(1+\bar{v}_{x}\right)$, can be identified with the final term in (A.23). Now, the flux of $q_{1}$ by the full cross-front velocity $\left(u_{1}=u_{1}^{g}+u_{1}^{a}\right)$ will necessarily contain a purely ageostrophic quantity [i.e. $u_{1}^{a}$ times part of (A.26)].

This ageostrophic flux can be related to the time rate-of-change of that part of the ageostrophic energy consistent with the geostrophic momentum approximation as follows. The terms neglected in the derivation of the upper layer geostrophic momentum equations (3.8a) and (3.8b) provide expressions which govern the ageostrophic velocity fields:

$$
\begin{aligned}
& u_{1_{t}}^{a}+\bar{v} u_{1_{y}}^{a}=0 \\
& v_{1_{t}}^{a}+\bar{v} v_{1_{y}}^{a}=0 .
\end{aligned}
$$

Multiplying (A.27) by $\overline{h_{1}}\left(1+\bar{v}_{x}\right)\left(u_{1}^{g}+u_{1}^{a}\right)$ and (A.28) by $\overline{h_{1}}\left(v_{1}^{g}+v_{1}^{a}\right)$ then adding yields

$$
\begin{aligned}
\overline{h_{1}}\left[\left(1+\bar{v}_{x}\right)\left(\frac{u_{1}^{a^{2}}}{2}\right)_{t}+\left(\frac{v_{1}^{a^{2}}}{2}\right)_{t}\right]+ & \overline{h_{1}}\left[\left(1+\bar{v}_{x}\right) u_{1}^{g} u_{1_{t}}^{a}+v_{1}^{g} v_{1_{t}}^{a}\right]= \\
& -\bar{v} \overline{h_{1}}\left[\left(1+\bar{v}_{x}\right) u_{1}^{g} u_{1_{y}}^{a}+v_{1}^{g} v_{1_{y}}^{a}\right]
\end{aligned}
$$

The terms on the right-hand-side of (A.29) may be replaced using the $y$ derivatives of (A.24) and (A.25):

$$
\begin{gathered}
v_{1_{y}}^{a}=u_{1_{y t}}^{g}+\bar{v} u_{1_{y y}}^{g}, \\
\left(1+\bar{v}_{x}\right) u_{1_{y}}^{a}=-\bar{v}_{x} u_{1_{y}}^{g}-v_{1_{y t}}^{g}-\bar{v} v_{1_{y y}}^{g} .
\end{gathered}
$$


The result is

$$
\begin{array}{r}
\bar{h}_{1}\left[\left(1+\bar{v}_{x}\right)\left(\frac{u_{1}^{a^{2}}}{2}\right)_{t}+\left(\frac{v_{1}^{a^{2}}}{2}\right)_{t}\right]+ \\
\overline{h_{1}}\left[\left(1+\bar{v}_{x}\right) u_{1}^{g} u_{1_{t}}^{a}+v_{1}^{g} v_{1_{t}}^{a}\right]= \\
-\bar{v} \overline{h_{1}}\left[\left(v_{1}^{g} u_{1_{y}}^{g}\right)_{t}+\left(\bar{v} u_{1_{y}}^{g} v_{1}^{g}\right)_{y}\right]
\end{array}
$$

which when averaged over the domain of the fluid is

$$
\begin{aligned}
\frac{\partial}{\partial t} \int \overline{h_{1}}\left[\left(1+\bar{v}_{x}\right) \frac{u_{1}^{a^{2}}}{2}+\frac{v_{1}^{a^{2}}}{2}\right] d A+ & \int \overline{h_{1}}\left[\left(1+\bar{v}_{x}\right) u_{1}^{g} u_{1_{\mathrm{t}}}^{a}+v_{1}^{g} v_{1_{t}}^{a}\right] d A= \\
& -\frac{\partial}{\partial t} \int \bar{v} \overline{h_{1}} v_{1}^{g} u_{1_{\mathrm{y}}}^{g} d A
\end{aligned}
$$

Since $v_{1}^{g}=\zeta_{1_{x}}$ and $u_{1}^{g}=-\zeta_{1_{y}}$, the term on the right-hand-side of this expression is just equal to the final term in (4.16). The terms on the left-hand-side represent the ageostrophic energy field, consistent with the geostrophic momentum approximation, whose existence was anticipated above from examination of the flux of part of the ageostrophic potential vorticity. [Note that the term $\int\left\{\left(1+\bar{v}_{x}\right) u_{1_{t}}^{g} u_{1}^{a}+v_{1_{t}}^{g} v_{1}^{a}\right\} d A$, absent on the lefthand-side, can be obtained by multiplying (3.8a) and (3.8b) by $\overline{h_{1}}\left(1+\bar{v}_{x}\right) u_{1}^{a}$ and $\overline{h_{1}} v_{1}^{a}$ respectively, in the derivation of the conservation of wave energy - see (A.14).]

A relationship between the time rate-of-change of $E_{2}$, defined in (4.9d), and the geostrophic momentum forms of the Reynolds stresses and the baroclinic conversion of energy is given by (4.13). However, the relationship of $\partial E_{2} / \partial t$ to the flux of potential vorticity is complicated by the presence of additional terms as sketched above. This is clearly a disadvantage of the geostrophic momentum approximation. To be able to relate the time rate-of-change of the wave energy to the change in time of the particle dispersion [see (4.20)], it was decided to use the full form of the wave energy (geostrophic plus ageostrophic) rather than just the geostrophic part. The full wave energy $\left(E_{2}^{\star}\right)$ is used throughout the analysis presented in the remainder of chapters 4 and 5 . 


\section{Conservation of Wave Momentum}

Conservation of wave $y$-momentum can be derived from the linearized perturbation equations (3.8) and (3.9) as follows. Multiplying (3.8b) by $-\zeta_{2}$ and $(3.8 \mathrm{c})$ by $\zeta_{1_{2}}$ and adding yields

$$
\begin{gathered}
-\left(\zeta_{2} \zeta_{1_{x}}\right)_{t}-\left(\zeta_{2} \bar{v} \zeta_{1_{x}}\right)_{y}-\left(1+\bar{v}_{x}\right) u_{1} \zeta_{2}+ \\
\left(u_{1} \overline{h_{1}}\right)_{x} \zeta_{1_{x}}+\overline{h_{1}} v_{1_{y}} \zeta_{1_{x}}=\zeta_{1_{y}} \zeta_{2} .
\end{gathered}
$$

A similar operation for the lower layer $\left[(3.9 \mathrm{~b}) \zeta_{2}+(3.9 \mathrm{c}) \zeta_{T_{x}}\right]$ gives

$$
\begin{gathered}
\left(\zeta_{2} \zeta_{T_{x}}\right)_{t}+u_{2} \zeta_{2}+\left(u_{2} \overline{h_{2}}\right)_{x} \zeta_{T_{x}}+ \\
\overline{h_{2}} v_{2 y} \zeta_{T_{x}}=-\zeta_{2} \zeta_{1_{y}}-\left(\frac{\zeta_{2}^{2}}{2}\right)_{y}
\end{gathered}
$$

Adding (A.30) to (A.31) and averaging over the domain of the fluid yields

$$
\begin{aligned}
& \frac{\partial}{\partial t} \int\left(\zeta_{T_{x}}-\zeta_{1_{x}}\right) \zeta_{2} d A=\int\left(1+\bar{v}_{x}\right) u_{1} \zeta_{2}-\left(u_{1} \overline{h_{1}}\right)_{x} \zeta_{1_{x}}-\overline{h_{1}} v_{1_{y}} \zeta_{1_{x}}- \\
& u_{2} \zeta_{2}-\left(u_{2} \overline{h_{2}}\right)_{x} \zeta_{T_{x}}-\overline{h_{2}} v_{2_{y}} \zeta_{T_{x}}
\end{aligned}
$$

The right-hand-side is rewritten using manipulations similar to those used in the derivation of the conservation of energy with the result

$$
\begin{aligned}
\frac{\partial M_{2}}{\partial t}= & \int{\overline{h_{1}}}^{2} u_{1}\left[\frac{\zeta_{1_{x x}}+\left(1+\bar{v}_{x}\right) \zeta_{1_{y y}}+Q_{1} \zeta_{2}}{\overline{h_{1}}}\right] d A+\frac{\partial}{\partial t} \int \overline{h_{1}} \zeta_{1_{x}} \zeta_{1_{y y}} d A+ \\
& \int{\overline{h_{2}}}^{2} u_{2}\left[\frac{\zeta_{T_{x x}}+\zeta_{T_{y y}}-Q_{2} \zeta_{2}}{\overline{h_{2}}}\right] d A+\frac{\partial}{\partial t} \int \overline{h_{2}} \zeta_{T_{x}} \zeta_{T_{y y}} d A,
\end{aligned}
$$

where $Q_{1}$ and $Q_{2}$ are defined by (4.5b) and (4.6b). The quantities in square brackets are just the perturbation potential vorticities in each layer given by (4.5c) and (4.6c) so that (A.32) becomes (4.21). The extra terms not included in the flux of $q_{1}$ and $q_{2}$ are related to part of the ageostrophic momentum field in a manner similar to that discussed above for energy. Again, these extra terms are removed by considering the full form of the wave momentum (geostrophic plus ageostrophic) as incorporated in (4.22). 


\section{References}

Allen, J.S., 1974. A simple model for stratified shelf flow fields with bottom friction. J. Phys. Oceanogr., 14, 1200-1214.

Bang, N.D., 1973. Characteristics of an intense ocean frontal system in the upwelling region west of Cape Town. Tellus, 25, 256-265.

Bane, J.M. and Y. Hsueh, 1980. On the theory of coastal-trapped waves in an upwelling frontal zone. J. Phys. Oceanogr., 10, 270-285.

Bane, J.M., 1980. Coastal-trapped and frontal-trapped waves in a baroclinic western boundary current. J. Phys. Oceanogr., 10, 1652-1668.

Bowman, M.J. and W.E. Esaias (Eds.), 1978. "Oceanic Fronts in Coastal Processes." Springer-Verlag, New York.

Breaker, L.C. and C.N.K. Mooers, 1986. Oceanic variability off the central California coast. Prog. Oceanogr., 17, 61-135.

Brink, K.H., 1982. The effect of bottom friction on low-frequency coastal trapped waves. J. Phys. Oceanogr., 10, 765-778.

Brink, K.H., 1983. The near-surface dynamics of coastal upwelling. Prog. Oceanogr., 12, 223-257.

Brink, K.H. and J.S. Allen, 1978. On the effect of bottom friction on barotropic motion over the continental shelf. J. Phys. Oceanogr., 8, 919-922.

Brink, K.H., D.C. Chapman and G.R. Halliwell, Jr., 1987. A stochastic model for wind-driven currents over the continental shelf. J. Geophys. Res., 92, 1783-1797.

Bryden, H.L., D. Halpern and R.D. Pillsbury, 1980. Importance of eddy heat flux in a heat budget for Oregon coastal waters. J. Geophys. Res., 85, 6,649-6,653.

Cairns, R.A., 1979. The role of negative energy waves in some instabilities of parallel flows. J. Fluid Mech., 92, 1-14.

Chapman, D.C., 1983. A note on the use of two-layer models of coastally trapped waves. Dyn. Atmos. Oceans, 8, 73-86.

Charney, J.G., 1949. The dynamics of long waves in a baroclinic westerly current. J. Meteorol., 4, 135-162.

Chia, F., R.W. Griffiths and P.F. Linden, 1982. Laboratory experiments on frontsPart II: The formation of cyclonic eddies at upwelling fronts. Geophys. Astrophys. Fluid Dyn., 19, 189-206.

Csanady, G.T., 1971. On the equilibrium shape of the thermocline in the shore zone. J. Phys. Oceanogr., 1, 263-270. 
Csanady, G.T., 1977. Intermittent "full" upwelling in Lake Ontario. J. Geophys. Res., 82, 397-419.

Curtin, T.B., 1979. Physical dynamics of the coastal upwelling frontal zone off Oregon. Ph.D. Dissertation, University of Miami, $338 \mathrm{pp}$.

Cushman-Roisin, B., 1986. Frontal geostrophic dynamics. J. Phys. Oceanogr., 16, 132-143.

Davis, R.E., 1985. Drifter observations of coastal surface currents during CODE: The method and descriptive view. J. Geophys. Res., 90, 4,741-4,755.

Davis, R.E., 1985. Drifter observations of coastal surface currents during CODE: The statistical and dynamical views. J. Geophys. Res., 90, 4,756-4,772.

de Szoeke, R.A. and J.G. Richman, 1981. The role of wind-generated mixing in coastal upwelling. J. Phys. Oceanogr., 11, 1534-1547.

de Szoeke, R.A. and J.G. Richman, 1984. On wind-driven mixed layers with strong horizontal gradients - A theory with application to coastal upwelling. J. Phys. Oceanogr., 14, 364-377.

Eady, E.T., 1949. Long waves and cyclone waves. Tellus, 1, 33-52.

Fjørtoft, R., 1950. Application of integral theorems in deriving criteria of stability for laminar flows and for the baroclinic circular vortex. Geophys. Publ, 17, 1-52.

Fjørtoft, R., 1951. Some results concerning the distribution and total amount of kinetic energy in the atmosphere. In "The Atmosphere and Sea in Motion." Rockefeller Institute and Oxford University Press, 509 pp.

Flament, P., L. Armi and L. Washburn, 1985. The evolving structure of an upwelling filament. J. Geophys. Res., 90, 11,765-11,778.

Garvine, R.W., 1979a. An integral hydrodynamic model of upper ocean frontal dynamics: Part I. Development and analysis. J. Phys. Oceanogr., 9, 1-18.

Garvine, R.W., 1979b. An integral hydrodynamic model of upper ocean frontal dynamics: Part II. Physical characteristics and comparison with observations. $J$. Phys. Oceanogr., 9, 19-36.

Garvine, R.W., 1980. The circulation dynamics and thermodynamics of upper ocean density fronts. J. Phys. Oceanogr., 10, 2058-2081.

Garvine, R.W., 1983. Stationary waves on oceanic density fronts. Deep-Sea Res., 30, 245-266.

Garvine, R.W., 1984. Propagating long waves on oceanic density fronts: An analytic model. J. Phys. Oceanogr., 14, 1590-1599. 
Gill, A.E. and E.H. Schumann, 1979. Topographically induced changes in the structure of an inertial coastal jet: Application to the Agulhas current. J. Phys. Oceanogr., 9, 975-991.

Griffiths, R.W., P.D. Killworth and M.F. Stern, 1982. Ageostrophic instability of ocean currents. J. Fluid Mech., 117, 343-377.

Griffiths, R.W. and P.F. Linden, 1982. Laboratory experiments on fronts-Part I: Density-driven boundary currents. Geophys. Astrophys. Fluid Dyn., 19, 159187.

Holopainen, E.O., 1961. On the effect of friction in baroclinic waves. Tellus, 13, 363-367.

Halpern, D., 1976. Structure of a coastal upwelling event observed off Oregon during July 1973. Deep-Sea Res., 23, 495-508.

Hartwig, E.O. and K.H. Brink, 1985. Coastal transition zone workshop report. Office of Naval Research, 67 pp.

Hayashi, Y.-Y. and W.R. Young, 1987. Stable and unstable shear modes on rotating parallel flows in shallow water. $J$. Fluid Mech., in press.

Hoskins, B.J., 1975. The geostrophic momentum approximation and the semigeostrophic equations. J. Atmos. Sci., 32, 233-242.

Hoskins, B.J. and F.P. Bretherton, 1972. Atmospheric frontogenesis models: Mathematical formulation and solution. J. Atmos. Sci., 29, 11-37.

Howard, L.N. and P.G. Drazin, 1964. On instability of parallel flow of inviscid fluid in a rotating system with variable Coriolis parameter. J. Math. Phys., 43, 83-99.

Huyer, A., 1983. Coastal upwelling in the California current system. Prog. Oceanogr., 12, 259-284.

James, I.A., 1984. A three-dimensional numerical shelf-sea front model with variable eddy viscosity and diffusivity. Continental Shelf Research, 3, 69-98.

Jury, M.R., 1984. Wind shear and differential upwelling along the SW tip of Africa. Ph.D. thesis, Univ. of Cape Town, 161 pp.

Kelly, K.A., 1985. The influence of winds and topography on the sea surface temperature patterns over the northern California slope. J. Geophys. Res., 90, 11,783-11,798.

Killworth, P.D., 1980. Barotropic and baroclinic instability in rotating stratified fluids. Dyn. Atmos. Oceans, 4, 143-184.

Killworth, P.D., 1983. Long-wave instability of an isolated front. Geophys. Astrophys. Fluid Dyn., 25, 235-258. 
Killworth, P.D. and M.F. Stern, 1982. Instabilities on density-driven boundary currents and fronts. Geophys. Astrophys. Fluid Dyn., 25, 235-258.

Killworth, P.D., N. Paldor and M.E. Stern, 1984. Wave propagation and growth on a surface front on a two-layer geostrophic current. J. Mar. Res., 42, 761-785.

Kosro, P.M., 1987. Structure of the coastal current field off northern California during the Coastal Ocean Dynamics Experiment. J. Geophys. Res., 92, 16371654.

Kubokawa, A., 1985. Instability of a geostrophic front and its energetics. Geophys. Astrophys. Fluid Dyn., 33, 223-257.

Kubokawa, A., 1986. Instability caused by the coalescence of two modes of a onelayer coastal current with a surface front. Journal of the Oceanographical Society of Japan, 42, 373-380.

Kuo, H.L., 1949. Dynamic instability of two-dimensional non-divergent flow in a barotropic atmosphere. J. Meteor., 6, 105-122.

Kuo, H.L., 1973. Dynamics of quasi-geostrophic flows and instability theory. Adv. Appl. Mech., 13, 247-330.

Lentz, S.J., 1987. A heat budget for the northern California shelf during CODE-2. $J$. Geophys. Res., in press.

Long, B., 1987. On the stability of steady ideal fluid flow. Ocean Modelling, 73, (unpublished manuscript).

Luther, M.E. and J.M. Bane, 1980. Coastal-trapped and frontal-trapped waves in a continuously stratified western boundary current. Rep. CMS-80-1, Curriculum in Marine Sciences, University of North Carolina, 77 pp.

Luther, M.E. and J.M. Bane, 1985. Mixed instabilities in the Gulf Stream over the continental slope. J. Phys. Oceanogr., 15, 3-23.

Marinone, S.G. and P. Ripa, 1983. Energetics of the instability of a depth independent equatorial jet. Geophys. Astrophys. Fluid Dyn., 30, 105-130.

Mechoso, C.R. and D.M. Sinton, 1981. Instability of baroclinic flows with horizontal shear along topography. J. Phys. Oceanogr., 11, 813-821.

Mooers, C.N.K., C.A. Collins and R.L. Smith, 1976. The dynamic structure of the frontal zone in the coastal upwelling region off Oregon. J. Phys. Oceanogr., 6, 3-21.

Narimousa, S. and T. Maxworthy, 1985. Two-layer model of shear-driven coastal upwelling in the presence of bottom topography. J. Fluid Mech., 159, 503-531. 
O'Brien, J.J., B.M. Woodworth and D.J. Wright, 1974. The Coho Project, II Environmental report. Joint report of Florida State University and Oregon State University, $116 \mathrm{pp}$.

Orlanski, I., 1968. Instability of frontal waves. J. Atmos. Sci., 25, 178-200.

Orlanski, I., 1969. The influence of bottom topography on the stability of jets in a baroclinic fluid. J. Atmos. Sci., 26, 1216-1232.

Orlanski, I. and M.D. Cox, 1973. Baroclinic instability in ocean currents. Geophysical Fluid Dynamics, 4, 297-332.

Paldor, N., 1983a. Linear stability and stable modes of geostrophic fronts. Geophys. Astrophys. Fluid Dyn., 24, 299-326.

Paldor, N., 1983b. Stability and stable modes of coastal fronts. Geophys. Astrophys. Fluid Dyn., 27, 217-228.

Paldor, N., 1986. Nonlinear waves on a coupled density front. Geophys. Astrophys. Fluid Dyn., 37, 171-191.

Pedlosky, J., 1964. The stability of currents in the atmosphere and the ocean: Part I. J. Atmos. Sci., 21, 201-219.

Pedlosky, J., 1974a. On coastal jets and upwelling in bounded basins. J. Phys. Oceanogr., 4, 3-18.

Pedlosky, J., 1974b. Longshore currents, upwelling and bottom topography. $J$. Phys. Oceanogr., 4, 214-226.

Pedlosky, J., 1974c. Longshore currents and the onset of upwelling over bottom slope. J. Phys. Oceanogr., 4, 310-320.

Pedlosky, J., 1978a. An inertial model of steady coastal upwelling. J. Phys. Oceanogr., 8, 171-177.

Pedlosky, J., 1978b. A nonlinear model of the onset of upwelling. J. Phys. Oceanogr., 8, 178-187.

Pedlosky, J., 1986. “Geophysical Fluid Dynamics," 2nd ed. Springer-Verlag, Berlin and New York, $710 \mathrm{pp}$.

Petrie, B., B.J. Topliss and D.G. Wright, 1987. Coastal upwelling and eddy development off Nova Scotia. J. Geophys. Res., in press.

Phillips, N., 1964. Energy transformations and meridional circulations associated with simple baroclinic waves in a two-layer model. Tellus, 6, 273-280.

Rayleigh, Lord, 1880. On the stability, or instability, of certain fluid motions. Proc. Lond. Math. Soc., 9, 57-70. [Also in Scientific Papers, 1, 474-487. Cambridge Univ. Press.] 
Richards, F.A. (Ed.), 1981. "Coastal Upwelling, Coastal Estuarine Sci. Ser.," Vol. 1. American Geophysical Union, Washington, D.C.

Ripa, P., 1983. General stability conditions for zonal flows in a one-layer model on the $\beta$-plane or the sphere. J. Fluid Mech., 126, 463-489.

Romea, R.D., 1977. The effect of friction and $\beta$ on finite-amplitude baroclinic waves. J. Atmos. Sci., 34, 1689-1695.

Rudnick, D.L. and R.E. Davis, 1987. Frontogenesis in mixed layer. J. Phys. Oceanogr., in press.

Simpson, J.H. and I.D. James, 1986. Coastal and estuarine fronts. In "Baroclinic Processes on Continental Shelves, Coastal Estuarine Sci. Ser.," Vol. 3. American Geophysical Union, Washington, D.C., 63-93.

Stern, M.E., 1980. Geostrophic fronts, bores, breaking and blocking waves. J. Fluid Mech., 99, 687-703.

Stern, M.E., J.A. Whitehead and B.-L. Hua, 1982. The intrusion of a density current along the coast of a rotating fluid. J. Fluid Mech., 123, 237-265.

Stevenson, M.R., R.W. Garvine and B. Wyatt, 1974. Lagrangian measurements in a coastal upwelling zone off Oregon. J. Phys. Oceanogr., 4, 321-336.

Taunton-Clark, J., 1982. CUEX ART Sea Surface Temperatures. Int. Rep. Sea Fish. Res. Inst. S. Afr., 175 pp.

Taunton-Clark, J., 1985. The formation, growth and decay of upwelling tongues in response to the mesoscale wind field during summer. In "South African Ocean Colour Experiment." Sea Fisheries Research Institute, Cape Town, 47-61.

Webster, I. and D. Holland, 1987. A numerical method for solving the forced baroclinic coastal-trapped wave problem of general form. J. Atmos. Ocean. Tech., 4, 220-226. 